\title{
?
}

TI 2020-053/V

Tinbergen Institute Discussion Paper

\section{Genetic Fortune: Winning or Losing Education, Income, and Health}

\section{Revision: November 2020}

Hyeokmoon Kweon ${ }^{1}$

Casper A.P. Burik ${ }^{1}$

Richard Karlsson Linnér ${ }^{1}$

Ronald de Vlaming ${ }^{1}$

Aysu Okbay ${ }^{1}$

Daphne Martschenko ${ }^{2}$

Kathryn Paige Harden ${ }^{3}$

Thomas A. DiPrete 4

Philipp D. Koellinger ${ }^{1}$

${ }^{1}$ Vrije Universiteit Amsterdam, Department of Economics

2 Stanford University, Stanford University Center for Biomedical Ethics

3 University of Texas at Austin, Department of Psychology

${ }^{4}$ Columbia University, Department of Sociology 
Tinbergen Institute is the graduate school and research institute in economics of Erasmus University Rotterdam, the University of Amsterdam and Vrije Universiteit Amsterdam.

Contact: discussionpapers@tinbergen.nl

More TI discussion papers can be downloaded at https://www.tinbergen.nl

Tinbergen Institute has two locations:

Tinbergen Institute Amsterdam

Gustav Mahlerplein 117

1082 MS Amsterdam

The Netherlands

Tel.: +31(0)205984580

Tinbergen Institute Rotterdam

Burg. Oudlaan 50

3062 PA Rotterdam

The Netherlands

Tel.: +31(0)10408 8900 


\title{
Genetic Fortune: Winning or Losing Education, Income, and Health
}

\author{
By HYEOKMOON KWEON \\ CASPER A.P. BURIK \\ RICHARD KARLSSON LINNÉR \\ RONALD DE VLAMING \\ AYSU OKBAY \\ DAPHNE MARTSCHENKO \\ K. PAige HARDEN \\ Thomas A. DiPrete \\ PhILIPP D. KOELLINGER*
}

\begin{abstract}
* Kweon: Department of Economics, Vrije Universiteit Amsterdam (h.kweon@vu.nl); Burik: Department of Economics, Vrije Universiteit Amsterdam (c.a.p.burik@vu.nl); Linnér: Department of Economics, Vrije Universiteit Amsterdam (r.karlssonlinner@vu.nl); de Vlaming: Department of Economics, Vrije Universiteit Amsterdam (r.devlaming@,vu.nl); Okbay: Department of Economics, Vrije Universiteit Amsterdam (a.okbay@vu.nl); Martschenko: Stanford University Center for Biomedical Ethics, Stanford University (daphnem@stanford.edu); Harden: Department of Psychology, University of Texas at Austin (harden@utexas.edu); DiPrete: Department of Sociology, Columbia University (tad61@columbia.edu); Koellinger: La Follette School of Public Affairs, University of Wisconsin-Madison and Department of Economics, Vrije Universiteit Amsterdam (koellinger@wisc.edu). Data analyses made use of the UK Biobank (UKB) resource under Application 11425. Genotype and phenotype data of the Health and Retirement Study (HRS) and the Wisconsin Longitudinal Study (WLS) can be accessed via the database of Genotypes and Phenotypes (dbGaP, accession no. phs000428.v2.p2 and phs001157.v1.p1.c1, respectively). HRS is supported by the National Institute on Aging (NIA U01AG009740, RC2AG036495, and RC4 AG039029). Researchers who wish to link genetic data with other HRS measures that are not in dbGaP, such as educational attainment, must apply for access from HRS. WLS has been supported principally by the National Institute on Aging (AG-9775, AG-21079, AG-033285, and AG-041868, R01 AG041868-01A1), with additional support from the Vilas Estate Trust, the National Science Foundation, the Spencer Foundation, and the Graduate School of the University of Wisconsin-Madison. Since 1992, data have been collected by the University of Wisconsin Survey Center. A public use file of data from the WLS is available from the University of Wisconsin-Madison, 1180 Observatory Drive, Madison, Wisconsin 53706 and at http://www.ssc.wisc.edu/WLSresearch/data/. The occupational wage data we created in the UKB, as well as the polygenic indices in the UKB sibling sample, the HRS, and the WLS are all shared via the standard data access mechanisms of these studies. We thank Matthew Keller and Luke Evans for providing the LD score estimates of the UK Biobank as well as Daniel Benjamin and Jonathan Beauchamp for comments and suggestions. We benefited greatly from the experiences and methods developed by the Social Science Genetic Association Consortium (SSGAC, https://www.thessgac.org/). Koellinger designed and oversaw the study and led the writing of the manuscript. Kweon was the lead analyst. Burik constructed polygenic scores and carried out analyses. De Vlaming and DiPrete wrote parts of the methods sections. Karlsson Linnér and Okbay contributed scripts and expertise for genetic analyses. All authors critically reviewed and edited the manuscript. Daphne Martschenko brought an adversarial collaborative perspective to this paper. Koellinger and Kweon made especially major contributions to the writing. The opinions expressed here are those of the authors and all mistakes are ours. Correspondence to Philipp D. Koellinger.
\end{abstract}


Genetic Fortune: Winning or Losing Education, Income, and Health

\begin{abstract}
We develop a polygenic index for individual income and examine random differences in this index with lifetime outcomes in a sample of $\sim 35,000$ biological siblings. We find that genetic fortune for higher income causes greater socio-economic status and better health, partly via intervenable environmental pathways such as education. The positive returns to schooling remain substantial even after controlling for now observable genetic confounds. Our findings illustrate that inequalities in education, income, and health are partly due the outcomes of a genetic lottery. However, the consequences of different genetic endowments are malleable, for example via policies that target education.
\end{abstract}

JEL codes: J00, I20, I10

Keywords: Income, education, health, inequality, heritability, genetics, polygenic index 
"The disposition to admire, and almost to worship, the rich and the powerful, and to despise, or, at least, to neglect persons of poor and mean condition is the great and most universal cause of the corruption of our moral sentiments."

Adam Smith (1759), The Theory of Moral Sentiments

\section{Introduction}

The origins, extent, and consequences of income inequalities differ across nations, regions, time and social systems (Kuznets 1955; Piketty and Saez 2003; Chetty and Hendren 2018; Corak 2013; Roine and Waldenström 2015). However, a universal fact is that parents influence the starting-points of their children by providing them with family-specific environments and by passing down a part of their genes. This phenomenon creates individual-specific social and genetic endowments that are due to luck in the sense that they are exogenously given rather than the result of one's own actions. Thus, inequalities of opportunity (Roemer and Trannoy 2015) can partly arise from the outcomes of two family-specific "lotteries" that take place during conception - a "social lottery" that determines who our parents are, and a "genetic lottery" that determines which part of their genomes our parents pass on to us. Inequalities in opportunity restrict the extent of intergenerational social mobility (Belsky et al. 2018; Jäntti and Jenkins 2015; Becker et al. 2018; Durlauf and Seshadri 2018) and limit how much credit people can claim for achievements such as their education or income (Roemer 1998; Rawls 1999). The relative importance of social and genetic luck has policy relevance because the extent to which people are willing to tolerate or endorse inequality partially depends on whether they perceive that disparity originates from differences in effort and choice (e.g., working hard) or from differences in circumstances that are outside of one's control (e.g., luck in the social or genetic lotteries). The empirical results suggest that inequality that can ultimately be traced back to luck may be perceived as unfair and people may favor redistributive policies more strongly if inequality is the result of luck rather than agency (Gromet, Hartson, and Sherman 2015; Cappelen et al. 2013; Almås et al. 2010; Alesina, Stantcheva, and Teso 2018; Alesina and La Ferrara 2005; Clark and D'Ambrosio 2015). It has even been suggested that GDP per capita as a measure of economic development should be replaced with a measure of the degree to which opportunities for income acquisition in a nation have been equalized (Roemer and Trannoy 2016).

If the outcomes of the genetic or social lottery influence economic outcomes, it can challenge common intuitions about the relative importance of luck and agency. For example, it is 
tempting to appraise good performance at work due to conscientiousness as rooted in individual agency. However, if genetics partly influence personality traits such as conscientiousness (Lo et al. 2016), luck and agency will be intertwined, and genetic fortune could be expected to affect outcomes throughout the life course not only via direct biological effects, but also through behavioral and environmental channels. It is important for science and policy to understand the extent to which genetic and social fortune contribute to inequality, the mechanisms that are at work, and whether and how the consequences of exogenously given endowments can be altered.

The current paper makes progress in this regard by using large-scale molecular genetic and family data to test the influence of genetic and family-specific endowments on income inequality and its consequences for health. Specifically, we develop a new polygenic index for individual income and exploit random differences between $\sim 35,000$ biological siblings in this index to estimate the consequences of the genetic lottery for income on a range of life-time outcomes. We show that the well-known gradient between socioeconomic status and health is partly rooted in exogenously given genetic and social endowments. Furthermore, we demonstrate that a substantial part of genetic luck for income and its link with health appears to operate via educational attainment and its accompaniments, i.e., environmental factors that are in principle malleable through policy interventions. Finally, we show that the effects of schooling on income remain strong and positive even when potential confounds from linear effects of common genetic variants are explicitly controlled for. Our results demonstrate the relevance of exogenously given genetic endowments for inequalities in income, education, and health. They also illustrate that the implications of the genetic lottery are not immutable because they operate at least partly via behavioral and environmental channels. Finally, our results emphasize the importance of education for inequality.

Our paper builds on recent work in social science genetics (Abdellaoui et al. 2019; Hill et al. 2016, 2019; Lee et al. 2018; Okbay et al. 2016; Rietveld et al. 2013) and applications of this work in economics. For example, Belsky et al. (2018) used family data to explore the links between a genetic index for educational attainment and various measures of social mobility. Furthermore, Barth, Papageorge, and Thom (2020) and Papageorge and Thom (2019) studied the associations between a genetic index for educational attainment and a variety of economic decisions and outcomes, without, however, using a within-family research design that would allow them to identify causal effects.

We accompany this article with a frequently asked questions (FAQ) document that explains in plain and simple language what we have done, what we found, what our results mean, and 
— importantly — what they do not mean (https://bit.ly/3f5TXoV). This FAQ document aims to address a wider audience of nonexperts in an effort to responsibly communicate scientific results, which is especially important given the dark history and abuses of social science genetics (Editors 2013; Nuffield Council on Bioethics 2002).

\section{I.A Background}

One approach researchers have used to quantify the relevance of luck due to genetic and family-specific endowments in the past are twin studies, which decompose observed differences in outcomes into genetic, family-specific, and residual variance components, leveraging the insight that monozygotic (MZ) twins are genetically (almost) completely identical, whereas dizygotic (DZ) twins have a genetic similarity of $\approx 50 \%$ (Plomin et al. 2012; Falconer and Mackay 2009). The identifying assumptions in classic twin studies include that MZ and DZ twins are different from each other only because of genetic reasons and not, for example, because parents treat MZ twin pairs systematically different from DZ twin pairs. Furthermore, classic twin studies assume that all genetic influences are additively linear and that parents are randomly matched rather than assorted based on similarity. Violations of these assumptions can lead to either upward or downward bias in the estimated variance components and have consequently sparked an extensive debate in the literature (Felson 2014; Zuk et al. 2012; Purcell 2002; Lerner 2006; Visscher, Hill, and Wray 2008). Additionally, the findings from twin studies are typically based on samples from specific Western, educated, industrialized, rich, and democratic (WEIRD) populations (Henrich, Heine, and Norenzayan 2010), thereby missing the importance of factors such as policies, culture, attitudes, institutions or economic development that do not vary much within the considered samples, but that can matter a great deal for differences between groups and over time.

Keeping these limitations in mind, the main conclusion from twin studies is that genetic differences account for a substantial part of the observed differences in income, educational attainment, or occupational choice in the samples analyzed (Polderman et al. 2015; Rietveld et al. 2013; Rowe, Vesterdal, and Rodgers 1998; Taubman 1976; Nicolaou and Shane 2010). For example, according to a meta-analysis of 10 studies based on 24,484 partly overlapping twin pairs, $52 \%(S E=0.03)$ of the variance in educational attainment can be attributed to genetic influences and $27 \%(S E=0.03)$ to family-specific environments (Polderman et al. 2015; Rietveld et al. 2013; Rowe, Vesterdal, and Rodgers 1998). The first study of this kind in economics (Taubman 1976) found a large influence of genetic and family-specific effects on 
earnings and years of schooling in a sample of white male twins who served in the U.S. Armed Forces during World War II. The article described these findings as "disturbing" given the author's inclination to accept socioeconomic inequalities due to "hard work and effort" much more than those arising from the contributions of one's parents.

Studies that considered genetic factors as potential contributors to socioeconomic inequality tend to trigger controversy, worry, and opposition (Comfort 2018). These concerns have to be taken seriously because misinterpretations of genetic influences and heritability estimates as measures of "purely biological" and "immutable" factors have been abused to justify ideologies about "natural rank orders" among individuals. This type of thinking has contributed to discrimination and some of the most horrifying atrocities in human history, including the Holocaust, involuntary sterilization programs, and state-sponsored violence targeting minorities and the poor (Ladd-Taylor 2020; Zimmer 2018; Kevles 1995). Unfortunately, these ideologies and dangers still exist today.

Viewing genetic influences as immutable factors that are independent from the environment is not only dangerous but also factually incorrect: the heritability of a trait puts no upper bound on the potential relevance of the environment (Goldberger 1978, 1979; Jencks 1980). Indeed, the heritability of a trait can even be entirely caused by environmental conditions. ${ }^{1}$ Furthermore, genetic influences on socioeconomic outcomes are most likely indirect, working via social and behavioral pathways that strongly depend on institutions, norms, policies, and incentives that are man-made and mutable (Jencks 1980). Genetic influences that work via environmental pathways, for example by selection into particular surroundings such as colleges, may lead to substantial disparities in outcomes such as income for environmental reasons that are everything but universal, perpetual, or "given by nature". As a result, genetic influences on socioeconomic outcomes can differ across divergent environments, making them neither inalterable nor purely biological factors. Thus, heritability estimates or genetic associations by themselves are uninformative about whether an environmental change such as a policy reform would affect an outcome or not. Rather, they are snapshots of a particular moment in time, a particular context, and most often of a particular ancestral population, one that is traditionally afforded higher income and education.

\footnotetext{
${ }^{1}$ For example, a hypothetical society that discriminates against people with red hair in college admissions would induce a heritability of educational attainment and a correlation between genes that influence hair pigmentation and college attendance, even though hair pigmentation may be orthogonal to academic aptitude (Jencks 1972).
} 
In response to some of these challenges, it has been suggested that "economists might do well to abandon the enterprise of determining the heritability of socioeconomic achievement measures" altogether (Goldberger 1978; Manski 2011). Although interest in the potential contributions of genetic factors to economic outcomes and behaviors has never entirely ceased (Sacerdote 2002; Zax and Rees 2002; Bowles and Gintis 2002; D. Cesarini et al. 2009; David Cesarini et al. 2010), most economists seem to have largely followed Goldberger's advice and turned their attention away from genetics and heritability estimates in the past four decades. ${ }^{2}$

However, genetic influences do not disappear just because one chooses to ignore them. Instead, genetic influences remain both a challenge and an opportunity for attempts to understand economic realities such as the origins and consequences of inequalities in income. First, genetic influences are a challenge because they may induce omitted variable bias in observational, nonexperimental studies. For example, a central issue for understanding the origins of inequality is to grasp the effects of education on income. One of the challenges in attempts to accurately estimate the returns to schooling are unobserved differences in "ability" that may have a genetic component (J. J. Heckman, Lochner, and Todd 2006). ${ }^{3}$ As a result, unaccounted genetic factors that are related to both educational attainment and income may lead to false conclusions about the extent to which differences in income can be attributed to schooling (DiPrete, Burik, and Koellinger 2018). Second, ignoring any source of variability of an outcome and relegating it to the error term of a regression necessarily leads to noissier, less precise estimates of the observed variables of interest. This phenomenon also holds for genetic sources of variability. Obviously, both uncertainty and bias can be serious obstacles in attempts to generate useful empirical insights.

Of course, these challenges are not new and economists already have potentially powerful tools to address them. For example, natural experiments and instrumental variable techniques can be used to identify causal effects, but they hinge on the availability of truly exogenous shocks that are relevant and measurable. Another popular way to address potential bias from unobserved heterogeneity is individual fixed-effects models. However, these models require

\footnotetext{
2 This development away from genetics in economics is in stark contrast to what happened in psychology, where estimating the heritability of traits and their co-heritability has been an active field of research since the 1970s that produced an extensive body of empirical evidence that can be succinctly summarized as "all human behavioral traits are heritable" (Turkheimer 2000), with an average heritability estimate of around $50 \%$ across all traits (Polderman et al. 2015).

3 "Ability" is often mentioned in economic studies on the returns to schooling, but it is also a historicallyburdened term that has been used to validate and carry out violent campaigns against the poor and racially-defined minorities at different points in history (Tabery 2015). This background contributes to the discomfort and caution applied to current genetics research (Roberts 2015). We use quotation marks in our mention of the term "ability" to recognize this historical legacy and the potentially misleading nature of this term.
} 
panel data featuring both regressors and regressands that vary among individuals over time, which restricts the type of questions one can ask. When genetic differences among people and their correlations with economic outcomes are observed directly, it opens up new opportunities to avoid unobserved variable bias and to obtain more accurate estimates of nongenetic influences (Harden and Koellinger 2020; Benjamin et al. 2012).

Furthermore, genetic data have two properties that make them particularly interesting for applied empirical work (Mills, Barban, and Tropf 2020). First, the genetic sequence of each person is fixed at conception and does not change throughout one's lifetime. Thus, reverse causality from behavior or environmental exposures to the genome can be ruled out. Therefore, genetic data provide researchers with the potential to construct noisy but exogenously given proxies for individual characteristics and outcomes that will emerge and change over the life course, allowing us to trace development paths. Second, each child is the result of a natural experiment that randomly mixes the genetic sequences of her biological parents. Thus, with the possible exception of monozygotic twins, all children who share the same biological parents exhibit random genetic differences. These exogenous shocks of the "genetic lottery" are a natural experiment that may be useful to identify causal relationships (Davies et al. 2019). Here, we provide an example of how random differences between siblings in a genetic score for income lead to inequalities in socioeconomic outcomes and health later in life and we begin to explore the possible mechanisms.

\section{Data}

\section{II.A. Genetic data}

The genome is encoded in a sequence of DNA (deoxyribonucleic acid) molecules. This sequence contains hereditary information that provides building instructions for all living organisms. In humans, the genome consists of 23 pairs of chromosomes, with one chromosome in each pair passed down by the father and one by the mother. Each chromosome is composed of two connected DNA strands that together resemble a twisted "ladder" (i.e., a double-helix). The "rails" of the "ladder" consist of a sugar-phosphate backbone and a nitrogenous base (adenine $[\mathrm{A}]$, cytosine $[\mathrm{C}]$, thymine $[\mathrm{T}]$, or guanine $[\mathrm{G}]$ ) is attached to each sugar-phosphate group. Together, these components construct a "nucleotide". The nitrogenous bases bind to each other in a strictly complementary way such that A always binds with $\mathrm{T}$ and $\mathrm{G}$ always binds with C, forming the "rungs" of the "ladder". The bases of the two copies of each chromosome may vary if father and mother passed down different variants. 
Human DNA consists of $\approx 3$ billion nucleotide pairs, the overwhelming majority of which are shared across individuals. Here, we study variations in nucleotide pairs in which some people carry a different base at a particular location (e.g., AT instead of GC). These so-called single nucleotide polymorphisms (SNPs) are the most common form of genetic variation that exists. Relatively common SNPs that vary among $>1 \%$ of humans make up less than $2 \%$ of all $\approx 3$ billion base pairs of human DNA (Auton et al. 2015), rendering these SNPs both informative about common genetic differences between people as well as relatively cheap ${ }^{4}$ and easy to measure (e.g., using saliva samples and high-throughput genotyping arrays) (Mills, Barban, and Tropf 2020). ${ }^{5}$

Because individuals have two copies of each chromosome, they typically have either two ATs, two GCs, or one AT and one GC at each position in their DNA. Therefore, SNPs can be numerically represented as count variables that indicate the number of copies of a chosen reference molecule (AT or GC), taking the values 0,1 , or 2 .

SNP data exhibit two types of correlations that must be taken into account. The first type consists of SNP correlations among the rows in the data (i.e., individuals) which increase if two individuals are related to each other and decrease with the number of generations that lie between them and their last common ancestor. While relatedness among individuals in a dataset can occur simply due to sampling multiple individuals from the same family, there can also be more subtle types of population structures underlying SNP data that can be traced back to shared ancestors many generations ago. Subgroups of the population that have different allele frequencies may also have different outcomes due to nongenetic factors such as cultural norms, policies, geographic environments, or economic circumstances, which can induce bias known as population stratification (Hamer and Sirota 2000). Thus, many research questions that rely on genetic data need to control for unobserved variable bias due to population structure (Price et al. 2006; Young et al. 2018, 2019).

Second, there is also a correlation structure among the SNPs themselves, i.e., the data columns. In molecular genetics, this is called linkage disequilibrium (LD) and it refers to the

\footnotetext{
4 The collection of a saliva sample, DNA extraction, and genotyping using a machine-readable array can currently be achieved for around $\$ 50$ or less.

5 In addition to the common SNPs analyzed here, other types of genetic variation exist such as rare and multiallelic SNPs or structural genetic variants including inversions, deletions, insertions, copy number variants, or translocations (Auton et al. (2015)). To the extent that these unobserved genetic variants are not or only weakly correlated with common SNPs, their influence cannot be detected well using SNP data. Thus, methods that use these data tend to underestimate the extent of genetic influences (Witte, Visscher, and Wray (2014)). To measure structural and rare genetic variants, full genome sequencing would be required, which is much more expensive than the array-based scans of common SNPs that we and the vast majority of all studies in human genetics rely on, and which would imply substantially smaller sample sizes.
} 
fact that genetic variants that are in close physical proximity to one another on a chromosome tend to be inherited together, creating persistent correlational patterns. LD is driven by several factors including biological mechanisms such as chromosomal crossover that happens during the formation of egg and sperm cells (i.e., meiosis), but also by mating patterns, selection, or migration events (Mills, Barban, and Tropf 2020). We detail below how we addressed potential biases from population structure and how we adjusted for LD in the construction of the genetic indices that are central for our applications.

\section{II.B. UK Biobank (UKB)}

The UKB is an ongoing population-based longitudinal study that was established to allow investigations of genetic and nongenetic factors that influence health outcomes in middle and old age. The UKB recruited 502,522 participants who were between 40-69 years old when they entered the study between 2006-2010 (Sudlow et al. 2015; Fry et al. 2017). All participants gave consent, answered questions, had physical measurements taken and provided samples of blood, urine and saliva at a baseline assessment center visit.

We use the molecular genetic data (see Appendix VI) and several available measures of SES of the UKB participants (standardized occupation codes, household income, educational attainment, and regional measures of socioeconomic status that were derived by the UKB from home locations and national statistics). We also use the digital health records of all participants, which are provided by UKB via continuously updated data linkage with the National Health Service (NHS). The NHS provides free medical treatment to all UK residents and is funded through general taxation. Thus, in contrast to other countries, access to medical treatment and the availability of digital health records in the UK is not a function of income or SES. Specifically, digital health records for England are available from hospital inpatient episodes (1996-2017), cancer registries (1971-2016), and death registries (2006-2018) ${ }^{6}$, providing clinical diagnoses for all instances according to the International Classification of Diseases (ICD; 9th or 10th revision), which defines the universe of diseases, disorders, injuries and other related health conditions in a comprehensive, hierarchical fashion (World Health Organization 2019). We examined all available hospital inpatient records, cancer episodes, and deaths for different types of disease using all major ICD chapters with a prevalence rate higher than $10 \%$ (16 in total). As an overall measure of health, we aggregate the available digital health records to examine whether participants had ever been hospitalized for any disease or diagnosed with

\footnotetext{
${ }^{6}$ See http://biobank.ndph.ox.ac.uk/showcase/exinfo.cgi?src=Data providers and dates
} 
any type of cancer. The available digital health records are left-censored, which prevents us from observing disease episodes from earlier periods where the participants were younger. It should therefore be kept in mind that our estimates with respect to disease occurrence and hospitalization are likely to be underestimated.

In addition, we use four proxies for health that are not subject to left-censoring and that are continuously distributed: body-mass-index (BMI), waist-to-hip ratio (WHR), blood pressure, and a measure of lung function (Global Burden of Disease Obesity Collaborators et al. 2017; Srikanthan, Seeman, and Karlamangla 2009; Huxley et al. 2010). Finally, we use a summary index of overall health that is a weighted sum of all binary and continuously distributed health indicators mentioned above. ${ }^{7}$ Table $\mathbf{A 1}$ provides a list of these variables and their definitions. In addition, Tables $\mathbf{A} \mathbf{2}$ and $\mathbf{A 3}$ show relevant descriptive statistics.

\section{II.C. Health and Retirement Study (HRS)}

The HRS is an ongoing longitudinal survey on health, retirement, and aging that is representative of the US population aged 50 years or older (Sonnega et al. 2014). The survey contains a wide range of socioeconomic outcomes, including income, educational attainment, working hours, and standardized job codes. Since 2006, data collection has expanded to include biomarkers and a subset of the participants has been genotyped (Weir 2013). We use the second release of the HRS genetic data here (see Appendix VI). Our primary outcome of interest in the HRS is hourly wages, which are constructed from self-reports of income and hours worked. We use a cleaned and harmonized dataset produced by the RAND corporation ${ }^{8}$, which includes twelve waves from 1992 to 2014 . We convert nominal wages into real wages using the consumer price index (base $=1982-84)$.

\section{II.D. Polygenic indices}

All heritable human behaviors are associated with very many genetic variants, each of which accounts for a very small percentage of the behavioral variability. This stylized fact is known

\footnotetext{
${ }^{7}$ The summary index of every health measure is constructed by following (Anderson 2008). This method takes a weighted average of standardized outcomes where weights are determined by the inverse of the correlation matrix. Outcomes highly correlated with each other are assigned less weight, while outcomes receive more weight if they are uncorrelated and therefore represent new information. The weights we used in our study are reported in Table A4.

${ }^{8}$ Health and Retirement Study, (RAND HRS Longitudinal File, version P) public use dataset. Produced and distributed by the University of Michigan with funding from the National Institute on Aging (grant number NIA U01AG009740). Ann Arbor, MI, 2017.

See https://www.rand.org/well-being/social-and-behavioral-policy/centers/aging/dataprod/hrs-data.html
} 
as the "Fourth Law of Behavior Genetics" (Chabris et al. 2015). Due to the sheer number of SNPs that are potentially relevant for human behavior and economic outcomes, it is difficult to incorporate them directly in an econometric model. Instead, an efficient and well-established way of exploiting the SNP data is to construct a polygenic index (PGI) that additively summarizes the effects of more than 1 million SNPs. Formally, a PGI $s_{i}$ is a weighted sum of SNPs:

$$
s_{i}=\sum_{j=1}^{J} \quad \hat{\beta}_{j} x_{i j}
$$

where $x_{i j}$ is individual $i$ 's genotype at SNP $j$. The weights $\hat{\beta}_{j}$ are estimated in a genome-wide association study (GWAS) (see Appendix III) which scans all measured genetic variations among people for associations with the outcome of interest. Since the number of SNPs $J$ is typically orders of magnitude greater than the number of individuals in the sample, it is impossible to fit all SNPs simultaneously in a multiple regression. Instead, the outcome is regressed on each SNP separately, resulting in $J$ regressions in total. Importantly, in order to avoid overfitting, the GWAS estimation sample does not include individuals for which a PGI is constructed.

PGI for several economic outcomes are already available, thanks to large-scale GWAS on traits such as educational attainment (Rietveld et al. 2013; Okbay et al. 2016; Lee et al. 2018), risk tolerance (Karlsson Linnér et al. 2019), subjective well-being (Turley et al. 2018), and household income (Hill et al. 2016, 2019). However, no PGI for individual income exists until now, despite the fact that individual income is one of the most central topics in economics and one of the most important proxies for well-being (Sacks, Stevenson, and Wolfers 2012; Stevenson and Wolfers 2013) and health throughout the lifecourse (Wilkinson and Marmot 2003; Chetty et al. 2016; Adler et al. 1994). The primary reason for this deficiency is that most datasets that contain genetic information have been collected for medical research purposes and lack measures of individual income. The few existing genetic datasets that do contain highquality measures of income are, unfortunately, too small to allow conducting statistically wellpowered GWAS on individual income (e.g. the Health and Retirement Study and the Wisconsin Longitudinal Study).

We remedy this issue by conducting GWAS on a good proxy for individual income, occupational wages, which we imputed from standardized occupation codes in the UKB, one of the largest existing genotyped datasets in the world. In essence, our imputation algorithm 
reflects the typical log wage of occupations in the UK, adjusted for demographic characteristics such as sex and age. Appendix I describes the procedure in detail. The income PGI that we created here adds to the growing array of polygenic indices that are useful for economists and other social scientists. Furthermore, a PGI for individual income is crucial for several of the analyses we present below, including our estimates of the returns to schooling.

Specifically, we follow a preregistered analysis plan (https://osf.io/rg8sh/) and conduct GWAS on occupational wages using 252,958 individuals in the UKB, excluding siblings and their close relatives to obtain an independent sample for follow-up analyses using the PGI. In Appendix III, we provide detailed descriptions of the GWAS and discuss the results of the GWAS on occupational wages with the full sample including the sibling sample $(N=282,963)$. In short, our GWAS on occupational wages identified 45 approximately independent genetic loci from 3,920 SNPs that are significant after Bonferroni correction for multiple testing $(p<$ $\left.5 \times 10^{-8}\right) .{ }^{9}$ The estimated effect size of each individual SNP is very small $\left(R^{2}<0.04 \%\right)$, which is consistent with previous GWAS results for socio-economic outcomes (Chabris et al. 2015; Rietveld et al. 2013; Lee et al. 2018; Hill et al. 2019). The effects of 1,197,148 SNPs are then aggregated into a PGI in the sibling sample. We take the correlations between SNPs into account by using a Bayseian approach that adjusts the estimated GWAS weights $\hat{\beta}_{j}$ with information about correlations between SNPs (Vilhjálmsson et al. 2015) (see Appendix IV). The resulting PGI is then standardized to have zero mean and unit variance. This PGI captures approximately $3 \%$ of the variation in occupational wages in the UKB sibling sample and $1 \%$ of self-reported wages in holdout samples from the U.S. (Table A7). ${ }^{10}$ For simplicity, we refer to this polygenic index as the "income PGI" below.

Our GWAS results for occupational wages are similar to those for educational attainment, which was previously studied in GWAS sample size of $N>1,000,000$ (Lee, Wedow, et al. (2018). The genetic similarity between occupational wages and educational attainment can be quantified by the so-called genetic correlation coefficient between both traits ${ }^{11}$, which is 0.923

\footnotetext{
${ }^{9}$ The summary statistics of the genome-wide association study (GWAS) presented here can be downloaded at https://osf.io/rg8sh/. These data are useful for many purposes such as constructing genetic indices, computing genetic correlations (Bulik-Sullivan et al. 2015), and for genetically informed study designs involving income (Harden and Koellinger 2020).

${ }^{10}$ The difference in $R^{2}$ of the PGI across samples is likely due to differences in heritability and the true genetic architecture of different measures of income (i.e. occupational wages versus self-reported wages) across different environments (i.e. UK versus US), see (de Vlaming et al. 2017).

${ }^{11}$ The genetic correlation between two traits quantifies the extent to which they share the same molecular genetic architecture, ranging from -1 to 1 (Okbay et al. 2016; Bulik-Sullivan et al. 2015; Harden and Koellinger 2020).
} 
$(S E=0.01)$. Thus, occupational wages and educational attainment are genetically very similar but not identical traits (see Appendix III). The genetic similarity between occupational wages and educational attainment can be exploited to improve the accuracy of the income PGI by applying a multivariate statistical method called Multi-Trait Analysis of Genome-wide association summary statistics (MTAG) (Turley et al. 2018). MTAG increases the accuracy of a PGI by "borrowing" information from GWAS estimates of genetically similar traits, which could also be obtained from partly or even completely overlapping GWAS samples. The MTAG approach substantially boosts the accuracy of the income PGI. For instance, the $R^{2}$ of the income PGI increases in the UKB holdout sample of siblings from $2.77 \%$ to $4.47 \%$ for occupational wages and from $0.66 \%$ to $1.40 \%$ for BMI when MTAG is used (Table A9).

\section{Statistical considerations}

Our main analysis examines the consequence of the genetic lottery for income on socioeconomic and health outcomes, taking advantage of the large sibling sample from the UKB. Consider the following baseline specification for outcome $y$ for individual $i$ from family $j:$

$$
y_{i j}=\delta s_{i j}+z_{i j}^{\prime} \theta+\alpha_{j}+e_{i j}
$$

where $s_{i j}$ is the PGI for income, $z_{i j}$ a vector of covariates, and $\alpha_{j}$ unobserved family-specific effects. In what follows, we discuss two important sources of potential bias when estimating the effects of the genetic lottery $(\delta)$ and how we address these issues.

\section{III.A. Confounds due to family environment}

Estimates of $\delta$ can be confounded due to the fact that the PGI only summarizes genetic associations, which are not necessarily the same as the causal genetic effects. The causal genetic effect can be defined as the average (counterfactual) change in an individual's outcome that would occur as a result of a ceteris paribus change of that individual's genotype at conception. In practice, however, GWAS are typically conducted in population samples and the obtained GWAS results and PGI can, and often do, contain environmental confounds, for example due to the environment that parents provide for their children (Kong et al. 2018). ${ }^{12}$

\footnotetext{
12 Another example is population stratification, i.e. environmental effects that correlate with more distant genetic ancestry that subgroups of the population share with each other such as cultural norms, policies,
} 
More generally, when $\operatorname{cov}\left(s_{i j}, \alpha_{j}\right) \neq 0$ and $\alpha_{j}$ is not specifically controlled for, estimates of $\delta$ will be inflated as a result of family-specific environmental conditions that influence $y_{i j}$. This bias is particularly relevant for socioeconomic outcomes (Kong et al. 2018; Lee et al. 2018; Young et al. 2018).

To break the link between $s_{i j}$ and $\alpha_{j}$, the natural experiment of meiosis can be exploited in a sample of siblings who share the same biological parents. During meiosis, the two copies of each parental chromosome are randomly combined and then separated to create a set of two gametes (e.g., two eggs or two sperm), each of which contains only one new, resampled copy of each chromosome. The resulting genetic differences between full siblings and dizygotic twins are therefore random and independent from family-specific ancestry and environmental factors that vary between families.

In a sample of siblings, the unobserved family-specific effects can simply be accounted for by including family fixed effects. Hence, a within-family regression will yield estimates of the coefficient for the PGI $(\delta)$ that are immune to parental genetic nurture and the uncontrolled population structure in GWAS that cannot be traced back to causal genetic effects. For this purpose, our main analysis relies on a hold-out sample of approximately 35,000 siblings from the UKB.

\section{III.B. Measurement error in the PGI}

Empirically estimated PGI are noisy proxies for "true" PGI that would capture the linear effects of all genetic variants in their entirety. The differences between the "true" and the available PGI are primarily due to two reasons. First, currently available genotyping technologies focus on common genetic variants, but they miss rare or structural genetic variants that are not highly correlated with the observed common variants (see footnote 7). For this reason, most empirical work in complex trait genetics is currently limited to studying the effects of common genetic variants, including this study. Second, GWAS estimates of the effect sizes of individual SNPs are noisy because they are obtained from finite sample sizes. The noise in the estimated effects of SNPs translates into noise in the PGI that is akin to classic (i.e. random) measurement error (Daetwyler, Villanueva, and Woolliams 2008; de Vlaming et al. 2017)

\footnotetext{
geographic environments, or economic circumstances (Hamer \& Sirota, 2000). GWAS typically try to address bias from population stratification by restricting samples to a relatively homogenous population, e.g. by limiting the study sample to individuals of European descent, and second by controlling for first 40 principal components from the SNP data. This is also the approach we followed here. These strategies help to some extent, but they are typically not sufficient to eliminate bias due to population stratification when socio-economic outcomes are studied in large GWAS samples (Abdellaoui et al. 2019; Haworth et al. 2019).
} 
which can be adjusted using instrumental variable regression (DiPrete, Burik, and Koellinger 2018). In our concrete example, we estimate that a PGI of all common genetic variants could potentially capture up to $\approx 10 \%$ of the variation in occupational wages, which is the share of variance in occupational wages that can be attributed to the combined linear effects of common genetic variants among UKB participants (See Appendix II). Thus, noisy GWAS estimates attenuate the accuracy of the currently available income PGI by more than $50 \%$.

To address attenuation bias due to measurement error, we use genetic instrument variable (GIV) regression (DiPrete, Burik, and Koellinger 2018), which constructs an instrument for the noisy PGI by randomly splitting the GWAS sample into two independent subsamples that allow for constructing two (even noisier) indicators of the PGI. Under the reasonable assumption that the error terms of both indicators are independent, one of them can be used as an instrument for the other to obtain coefficient estimates that are corrected for measurement error.

More formally, define a PGI $s_{i}=s_{i}{ }^{*}+u_{i}$, where $s_{i}{ }^{*}$ is the true PGI and $u_{i}$ is additive measurement error. Because the PGI is a linear combination of SNP effects, we can write $u_{i}=$ $\mathbf{x}_{i}^{\prime}(\mathbf{b}-\boldsymbol{\beta})$, where $\mathbf{x}_{i}$ is the vector of SNP data for individual $i, \boldsymbol{\beta}$ is the vector of true SNP effects, and $\mathbf{b}$ is the vector of estimated SNP effects. That is, the PGI can be decomposed into a true part and the contribution from the estimation error in the GWAS (i.e., $\mathbf{b}-\boldsymbol{\beta}$ ).

Suppose that we generate two PGI, by randomly splitting the GWAS sample into two independent subsamples to obtain two estimates of $\boldsymbol{\beta}$, where $s_{i}{ }^{(1)}$ is constructed using the estimate from one subsample and $s_{i}{ }^{(2)}$ using the estimate from the other subsample, where the additive measurement error in $s_{i}^{(2)}$ is denoted by $u_{i}^{(2)}$.

Now, if we are to use $s_{i}{ }^{(2)}=s_{i}{ }^{*}+u_{i}{ }^{(2)}$ as an instrument for $s_{i}{ }^{(1)}, s_{i}{ }^{(2)}$ must capture the true PGI term $s_{i}{ }^{*}$ only in the first stage regression. This implies that the noise terms $u_{i}{ }^{(1)}$ and $u_{i}{ }^{(2)}$ of the two PGI must be uncorrelated with each other. Thus, the estimation error of GWAS, $\mathbf{b}$ - $\boldsymbol{\beta}$, cannot be correlated across the two subsamples, so that $\operatorname{Cov}\left(u_{i}{ }^{(1)}, u_{i}^{(2)}\right)=0$. In practice, the two most important steps that need to be taken are (1) excluding genetic relatives from all subsamples and (2) adding fairly rigorous controls against population structure to the GWAS. To the extent that $\operatorname{Cov}\left(u_{i}{ }^{(1)}, u_{i}{ }^{(2)}\right)=0$ holds, using one PGI as an instrument for the PGI in a two-stage least squares regression will yield effect size estimates for the PGI that are no longer attenuated by finite GWAS sample sizes (DiPrete, Burik, and Koellinger 2018). However, even this correction of measurement error in PGI due to finite GWAS sample sizes does not address the fact that the influence of rare and structural genetic variants that are not well tagged by 
current genotyping arrays remain unobserved. Therefore, estimates of the effects of the genetic lottery that we report below are lower bounds for the influences of all genetic variants.

To obtain GWAS results for GIV analyses, we split the UKB GWAS estimation sample randomly into two subsamples, each containing 126,478 individuals. The subsamples have the same male-female ratio and the individuals in each sample are genetically related to those in the other sample with no more than first degree cousins or great-grandparents. We re-conducted a GWAS of occupational wages on these two subsamples and constructed two PGI for the sibling sample to use for GIV analyses. Note that these GIV PGI are not augmented with the GWAS results of educational attainment using MTAG.

\section{The SES-health gradient in the UK Biobank}

It is well-known that people with high SES also tend to live longer and healthier lives than those with lower SES (Wilkinson and Marmot 2003; Stringhini et al. 2017b; Piotrowska et al. 2015; Chetty et al. 2016). Natural experiments show that higher education has a positive causal effect on health (Grossman 2000, 2006). However, studies looking at income and health have produced mixed results about causal effects and come with many methodological challenges (Kawachi, Adler, and Dow 2010; O’Donnell, Van Doorslaer, and Van Ourti 2015).

The UK Biobank offers a unique opportunity to gain additional insights into the relationship between SES and health thanks to its broad coverage of the UK population; its large sample size, which includes one of the largest samples of genotyped siblings in the world; as well as the availability of detailed health records from assessment center visits and digital health records that are continuously updated and that span the entire universe of medical diagnoses. In addition to descriptive analyses of the SES-health gradient for a variety of health outcomes, this particular type of data also allows us to estimate the extent to which exogenously given endowments from the social and the genetic lottery drive the relationships between SES and health. As a first step, we conduct a family fixed-effects analysis in the sibling sample that allows us to control for the outcomes of the social lottery (i.e., the parental environment that both siblings share) and a part of the genetic lottery (i.e., the genetic similarity of siblings that is due to their descent from the same biological parents). The remaining differences in SES and health outcomes between siblings are the result of their random genetic differences as well as unique environmental influences that are unrelated to their shared genetic endowments.

Table 1 shows the relationship between SES, approximated by having a college degree and occupational wage, and health outcomes in the UKB. The first five rows of the table show 
estimates for the gradient with continuous proxies of health that include the waist-to-hip ratio, BMI, blood pressure, lung function, as well as the summary index for health. The results imply strong health advantages for people with higher SES. For example, a ten percent increase in occupational wages is associated with $\approx 0.12$ decrease in BMI (95\% CI: 0.09-0.34). The same picture emerges for the digital health records that were grouped into specific disease categories: Individuals with higher occupational wages and a college degree exhibit a lower tendency for severe disease outcomes that would require hospitalization (ever hospitalized). High SES is also associated with a lower likelihood of being diagnosed with all major disease categories, with the exception of neoplasms and cancers. The association between SES and health outcomes is particularly strong for endocrine, nutritional, and metabolic diseases; mental, behavioral, and nervous system disorders; and diseases of the circulatory and digestive systems. For example, having a college degree decreases the risk of ever being hospitalized for diseases of the circulatory system by $\approx 8$ percentage points (95\% CI: 6.12-10.10). These estimates are a lower bound of the SES-health gradient because the well-known healthy volunteer bias in the UK Biobank attenuates the estimates (Fry et al. 2017).

The results in Table 1 also clearly demonstrate that exogenously given family-specific endowments are responsible for the majority of the gradient between SES and health. In particular, when we control for family fixed effects, all estimated coefficients between SES and health are closer to zero and only the associations of SES with circulatory system disorders, waist-hip-ratio, lung function, and the summary index across all health outcomes remain statistically distinguishable from zero. The substantial contributions of family-specific genetic and environmental effects that are outside of one's control emphasize moral concerns about these observed health inequalities (Gromet, Hartson, and Sherman 2015; Cappelen et al. 2013; Almås et al. 2010; Alesina, Stantcheva, and Teso 2018; Alesina and La Ferrara 2005).

\section{Consequences of the genetic lottery for income}

We now turn to the consequences of the genetic lottery based on the random differences between siblings in their polygenic index for income. Our approach allows us to examine the causal impact of the genetic lottery for income on lifetime outcomes in the present-day UK.

There are 18,807 genetic sibling groups in the UKB (38,698 individuals). Our analyses are restricted to pairs that have the respective outcome variables available for both individuals, ${ }^{13}$

\footnotetext{
13 Only 1,003 sibling groups have more than 2 members. We dropped sibling groups if more than two siblings were available for a given outcome.
} 
leading to varying sample sizes between 8,780 and 17,633 pairs per outcome. We regressed each of the SES and health outcomes on the income PGI and covariates. ${ }^{14}$ For each outcome, we estimated the regression with and without family fixed effects. In the OLS estimation, the MTAG income PGI is used, whereas GIV estimation uses the ordinary income PGI estimated from the UKB subsamples. ${ }^{15}$ All PGIs are standardized to have zero mean and unit variance. We adjusted for multiple hypothesis testing using Holm's method (Holm 1979) in each set of analyses. $^{16}$

Figure A1 shows the distribution of the sibling difference in the MTAG income PGI in absolute value. Most of the sibling pairs exhibit a very small difference. ${ }^{17}$ Half of the sibling pairs have a difference in income PGI values smaller than 0.63 , measured in standard deviations of PGI in the sibling sample. The results of our within-family PGI analyses are presented in Table 2 and 3. The OLS estimates reported in the first column of Table 2 and $\mathbf{3}$ demonstrate that the MTAG income PGI is associated with all socioeconomic and almost all health-related outcomes we investigated. Furthermore, as reported in the third column, GIV regression estimates, which correct for measurement error in the PGI are typically twice as large as their corresponding OLS estimates.

Across the board, we find that a higher income PGI is associated with more favorable lifetime outcomes including higher educational attainment, higher occupational wages, living in a better neighborhood, a lower BMI and waist-to-hip ratio, lower blood pressure, a lower chance of having ever been hospitalized, and a lower probability of being diagnosed with all disease categories in the digital health records that that we investigated, again with the exception of cancer and neoplasms (Figure A2). When we correct for the attenuation bias in our results due to the measurement error in the PGI using GIV regression (but before we control for family fixed effects), our estimates show that a one-standard-deviation increase in the genetic propensity for higher income is associated with a $15 \%$ increase in occupational wages, a 7 percentage-point-increase in the likelihood of having a university education, an almost onepoint-decrease in BMI, and a 4-percentage-point decrease in the likelihood of ever being

\footnotetext{
${ }^{14}$ See the note in Table 2 and 3 for the included covariates.

15 This is because the GWAS results for educational attainment are from a meta-analysis of many cohorts.

${ }^{16}$ Holm's method controls the familywise error rate like Bonferroni correction, while it offers a uniformly more powerful correction by sequentially adjusting rejection criteria.

$1722 \%$ of the variation in the MTAG income PGI comes from within-family differences, while $78 \%$ comes from between-family variation. The correlation of a genotype between two siblings is 0.5 in expectation, which implies that $25 \%$ of the variation in the PGI is due to within-family differences in expectation. However, in the presence of assortative mating, the PGI of siblings can be more similar to each other than in expectation, which can lower the share of the within-family variation to below $25 \%$.
} 
hospitalized for the given age. Thus, the phenotypic associations between SES and health are mirrored in the associations between the PGI for income and health.

This pattern of results is consistent with the finding that measures of SES such as educational attainment show pervasive and often substantial genetic correlations with health outcomes that range between -0.3 for Alzheimer's disease, depressive symptoms, and body fat percentage to 0.6 with Mother's age at death (Bulik-Sullivan et al. 2015; Harden and Koellinger 2020), illustrating that health and SES are also tightly intertwined at a genetic level.

However, a substantial part of the correlations between PGI for socioeconomic outcomes and disease is likely to be due to indirect genetic effects such as genetic nurture (Kong et al. 2018) or subtle forms of population stratification such as correlations between gene frequencies and neighborhood characteristics that are also correlated with SES and health outcomes (Abdellaoui et al. 2019; Haworth et al. 2019). When comparing our OLS estimates of the coefficient for income PGI with and without family fixed-effects, we observe that the withinfamily effects are typically halved (Figure A2). For instance, the estimated effect of a one standard deviation increase in the PGI for log occupational wage per hour decreases from 0.074 (95\% CI: $0.07-0.08)$ to 0.046 (95\% CI: 0.03-0.06) after controlling for family fixed-effects. Likewise, the estimate of a one standard deviation increase in the income PGI with family fixed effects implies a 0.29 reduction in BMI (95\% CI: 0.16-0.41), while it is estimated to be a 0.52 reduction without family fixed effects (95\% CI: 0.51-0.62). However, even with the smaller point estimates and the larger standard errors from within-family analyses, we still find statistically significant associations of the income PGI with all socioeconomic outcomes we investigated as well as with BMI, waist-to-hip ratio, and diseases of the musculoskeletal system and connective tissues. Thus, approximately one half of the observed associations between our income PGI, socioeconomic attainment, and health outcomes in late adulthood are due to random genetic differences between siblings.

Finally, combining the GIV regression with family fixed-effects allows us to estimate the combined linear causal effects of common SNPs while adjusting the PGI for measurement error. Despite substantially larger standard errors of the point estimates due to the two-stage least squares approach of the GIV regression, we find effects of the genetic lottery for a number of outcomes that are statistically distinguishable from zero, including occupational wages, household income, regional income, years of schooling, and having a college degree. For example, a one-standard-deviation increase in income PGI is estimated to increase the chance of obtaining a college degree by 14.5 percentage points (95\% CI: 10.4-18.6) and an annual household income greater than $£ 52,000$ by 9.2 percentage points (95\% CI: $4.3-14.1)$. Although 
none of the health and disease measures is statistically significant in GIV fixed-effects estimations due to lower estimation precision, the point estimates are very similar to the statistically significant OLS estimates in the first column of Tables 2 and $\mathbf{3}$.

We repeated these analyses with a PGI for income that was not augmented by using MTAG (Table A12) and obtained qualitatively similar results, but with smaller point estimates in OLS regression due to the larger measurement error in the non-augmented PGI. We also conducted these analysis using a PGI for educational attainment (Table A13), with very similar results. Interesting, the PGI for educational attainment remains associated with health outcomes even after we add controls for actually achieved educational attainment, albeit with smaller effect sizes.

These results are inline with findings from Selzam et al. (2019) who compared PGI predictions within- and between-family for standardized test scores, IQ, and health-related outcomes using the Twins Early Development Study from the UK. They found that PGI are still predictive within-family while within-family estimate sizes for the PGI are typically smaller than between-family estimates. The differences are particularly large for standardized test scores, for which family background seems to play a more important role. These differences tend to disappear once parental socioeconomic variables are controlled for, suggesting that it is mainly the family's socioeconomic status that confounds the PGI. Similar findings for within-family estimates were also reported by Belsky et al. (2018).

\section{Decomposition of the genetic lottery effects}

The previous section demonstrated that the genetic lottery for income has incontrovertible consequences for a broad range of life-time outcomes. However, as mentioned in section I.A, these genetic influences do not imply purely biological mechanisms, nor do they imply that policy interventions are doomed to be unsuccessful (Goldberger 1979; Jencks 1980; Harden and Koellinger 2020). To illustrate these important points, consider an intervenable pathway $m_{i j}$ for individual $i$ in family $j$ through which the genetic lottery for income may affect an outcome such as health (e.g. access to favourable environmental conditions such as highquality health care, healthy nutrition, or clean air and water). This intervenable pathway $m_{i j}$

can be added to model (2) and an auxiliary regression can be conducted, where $m_{i j}$ is the dependent variable:

$$
y_{i j}=\tilde{\alpha}_{j}+\tilde{\delta}_{1} s_{i j}+\tilde{\delta}_{2} m_{i j}+z^{\prime}{ }_{i j} \tilde{\theta}+\tilde{e}_{i j}
$$




$$
m_{i j}=\alpha^{m}+\gamma s_{i j}+z_{i j}^{\prime} \theta^{m}+e^{m}{ }_{i j}
$$

Then, the coefficient of the PGI $\delta$ from the model (2) can be written as:

$$
\delta=\tilde{\delta}_{1}+\gamma \cdot \tilde{\delta}_{2}
$$

Therefore, the effect of genetic lottery $\delta$ can be decomposed into the effect working via pathway $m_{i j}\left(\gamma \cdot \tilde{\delta}_{2}\right)$ and the residual effect that does not work via that pathway $\left(\tilde{\delta}_{1}\right)$. Estimates of each parameter $\left(\tilde{\delta}_{1}, \tilde{\delta}_{2}, \gamma\right)$ can simply be obtained by estimating the equations (3) and (4) separately and the pathway effect $\left(\gamma \cdot \tilde{\delta}_{2}\right)$ can be estimated as the product of estimates of $\gamma$ and $\tilde{\delta}_{2}$. The standard errors can be computed by the delta method.

As an empirical illustration, we focus on having a college degree as an example of $m_{i j}$. Colleges are social institutions that have admission policies, procedures, and graduation requirements that are shaped by their decision makers and that can be influenced by policy. In this sense, colleges are intervenable institutions. They create value by giving their attendees access to potentially valuable assets (e.g. knowledge and skills). They can also bestow advantages on their attendees by serving as a signalling mechanism for potential employers that have imperfect information about job applicants (Michael 1973; Arcidiacono, Bayer, and Hizmo 2010). Of note, colleges remain intervenable institutions independent from how heritable it is to have a college degree and to which extent the genetic architecture of having a college degree is shared with other lifetime outcomes such as income or health. In fact, policy interventions could change both the heritability of having a college degree as well as it's molecular genetic architecture dramatically. For example, a policy that randomly assigns people to college could lower the heritability of educational attainment substantially. Alternatively, a policy that forbids men to go to college would lead to a perfect correlation between having a college degree and whether an individual has one or two X chromosomes, without any meaningful biological mechanism that would stop men from going to college in a different environment. And yet, as long as colleges grant some advantages to their attendees that have health benefits, any genetic variant that is associated with college attendance would also have an indirect health benefit, but these health effects of genes could in principle be intervened upon.

To increase statistical power, we limit our empirical analyses to five lifetime outcomes that are continuously distributed and available for many UKB participants (occupational wages, BMI, waist-to-hip ratio, blood pressure, and lung function). The participants were at least aged 
40, with the mean age of 57, when they were assessed for these measures. This limits concerns about potential reverse causality of these outcomes on college attendance.

While we can interpret the total effects of the genetic lottery as causal in the within-family model, this is not the case for the decomposed effects. In order for the intervenable pathway $m_{i j}$ to be causal, it would be required that the PGI is exogenous with respect to both the lateadulthood outcomes and college education conditional on the covariates and, second, that having a college degree is exogenous with respect to the outcomes later in life conditional both on the PGI and the covariates. ${ }^{18}$ Whereas the first part of these assumptions is plausibly satisfied given the random distribution of the PGI between siblings, the second part is likely to be violated in practice. In particular, this condition requires that there is no unobserved variable that affects both the late-adulthood outcomes and college education, which is clearly unrealistic. Having a college degree can be expected to affect many health-relevant circumstances, including income, neighborhood quality, and lifestyle-related choices that could influence health (e.g., smoking, alcohol consumption, diet, and physical activity) despite conditioning on family fixed effects. Therefore, the decomposed effects we estimate here do not illustrate the causal mechanism of the genetic effect. Instead, the results reported in Table 4 illustrate that the genetic lottery for income affects occupational wages and health partly via college education and its unobserved accompaniments - videlicet pathways that can be environmentally intervened upon (Barcellos, Carvalho, and Turley 2018).

For occupational wages and all the objective health outcomes that we examined, we observe that the effect of the MTAG income PGI that operates via college education and its accompaniments is statistically significant after Bonferroni correction for multiple testing. A one-standard-deviation increase in the PGI boosts the probability of attaining a college degree by up to 14.5 percentage points (Table 2), and having a college degree is in turn associated with lower waist-to-hip ratio, BMI, and blood pressure as well as better lung function and higher occupational wages. The intervenable pathway that is approximated by having a college degree accounts for almost $35 \%$ of the total effect of the income PGI on occupational wages. For the health outcomes, $9 \%-29 \%$ is accounted for by the indirect path, with the lowest indirect effect for BMI and the highest for lung function. Obviously, these are lower bound estimates for how much of the effect of the genetic lottery could be intervened upon because the residual effects of the PGI could include other $m_{i j}$ that imperfectly correlated with having

\footnotetext{
18 This is the same logic as the sequential ignorability assumption in causal mediation analysis (Imai, Keele, and Yamamoto 2010; J. Heckman and Pinto 2015)
} 
a college degree. While the estimated residual effects of the PGI on the health outcomes in Table 4 are often too noisy to draw a clear statistical inference, we find statistically significant effects of the $m_{i j}$ pathway that is approximated by having a college degree in every case.

Thus, educational attainment and its accompaniments play a crucial role in the relationship between genetic fortune for income and health outcomes in later life. Thus, the genetic associations we report here clearly do not imply biological determinism.

\section{Returns to schooling}

The results above show a clear relationship between genetic predisposition (i.e., the results of genetic lottery), educational attainment, and income. This reinvigorates the much-debated question in economics of how sensitive estimates of the returns to schooling are to hitherto unobserved genetic confounds. Could it be that the strong, positive relationship between schooling and income is biased upwards by unobserved differences in family backgrounds and "ability" 19 that are at least partially rooted in genetic factors (Mincer 1958; Griliches 1977; J. J. Heckman, Lochner, and Todd 2006)? We address this question for the first time with an explicit control for potential confounds from common genetic variants that may influence both education and income. Specifically, we use data from the HRS and our (nonaugmented) PGI for income and apply GIV regression to correct for measurement error in the PGI (DiPrete, Burik, and Koellinger 2018).

The coefficient we estimate is not the ex ante expected rate of return, which depends on psychic and financial costs of education, expected tax rates, expected number of working years after completing school, expected option values of additional years of education, and other information known to the economic agent at the time schooling decisions are being made (J. J. Heckman, Lochner, and Todd 2006). The approach we take here is much more humble. It addresses the question of whether the ex post average growth rate of income with respect to schooling is potentially biased by hitherto unobserved linear effects of common genetic variants. Nevertheless, we use the more well-known phrase "returns to schooling" throughout the rest of the paper to improve understandability.

We pool individual observations in the HRS across the waves spanning from 1992 to 2014, which provides us with a weighted average of cross-sectional estimates, and we estimate a standard Mincer equation. We also consider a more flexible specification to capture potentially nonlinear returns to higher education by including a dummy variable for college education as

\footnotetext{
${ }^{19}$ See footnote 5 .
} 
well as an interaction term for having a college degree and years of schooling. As relevant proxies for family backgrounds, we also add controls for years of schooling for both parents.

As a measure of genetic confounds, we would ideally want to have a PGI that captures only directly pleiotropic effects on educational attainment and individual income (rather than genetic effects that are mediated by educational attainment). Thus, a PGI for educational attainment cannot be used as a control variable in this context because it would remove the covariation in years of schooling and income that we intend to identify. However, it is possible to obtain reasonable upper and lower bounds of the relationship between education and income conditional on genetic effects using GIV regression (DiPrete, Burik, and Koellinger 2018).

More specifically, the GIV regressions of the returns to schooling estimate the following equations with two-stage least squares:

$$
\begin{aligned}
& y_{i}=\beta_{0}{ }^{c}+\beta_{1}{ }^{c} e d u_{i}+\beta_{2}{ }^{c} s_{y \mid e d u, i}{ }^{(1)}+z^{\prime}{ }_{i} \gamma^{c}+e_{i}{ }^{c} \\
& s_{y \mid e d u, i}{ }^{(1)}=\delta_{1}{ }^{c} s_{y, i}{ }^{(2)}+\delta_{2}{ }^{c} e d u_{i}+z^{\prime}{ }_{i} \theta^{c}+u_{i}{ }^{c} \\
& y_{i}=\beta_{0}{ }^{u}+\beta_{1}{ }^{u} e d u_{i}+\beta_{2}{ }^{u} s_{y, i}{ }^{(1)}+z^{\prime}{ }_{i} \gamma^{u}+e_{i}{ }^{u} \\
& s_{y, i}{ }^{(1)}=\delta_{1}{ }^{u} s_{y, i}{ }^{(2)}+\delta_{2}{ }^{u} e d u_{i}+z^{\prime}{ }_{i} \theta^{u}+u_{i}{ }^{u}
\end{aligned}
$$

where GIV-C and GIV-U are described by equations (6) and (7) as well as (8) and (9), respectively. $y_{i}$ denotes $\log$ hourly wages and $e d u_{i}$ the years of schooling for individual $i$. $s_{k, i}{ }^{(1)}$ is a PGI summarizing the GWAS effects of outcome $k$ estimated with the first subsample, where outcome $k$ is log hourly wage $(y)$ for GIV-U while it is the log hourly wage conditional on years of schooling $(y \mid e d u)$ for GIV-C. $s_{y, i}{ }^{(2)}$ is a PGI constructed from a GWAS on hourly wage estimated with the second subsample. $z_{i}$ is a vector of control variables and $e_{i}$ and $u_{i}$ are error terms.

Extensive simulations under a wide variety of conditions found the GIV-U estimate to be downwardly biased and the GIV-C estimate to be upwardly biased as long as no environmental endogeneity was present (DiPrete, Burik, and Koellinger 2018). Thus, when taken together, the use of GIV-U and GIV-C will generally produce bounds on the true effect of the exposure $(e d u)$. Moreover, in the simulations performed by DiPrete, Burik, and Koellinger (2018), the upward bias of GIV-C was always smaller than the upward bias in OLS.

Intuitively, GIV-U provides the lower bound for the relationship between education and income conditional on the currently observed linear SNP effects because the PGI that is used as a control in this regression also captures genetic effects on income that work via education. 
On the other hand, GIV-C provides an upper bound because although it mimics a regression of income on education conditional on all SNPs, it does so only imperfectly (see Table 1 in DiPrete et al. (2018)) and some of the bias due to direct pleiotropic effects of SNPs on education and income may remain in the estimates.

When environmental sources of endogeneity are present, of course, the GIV-U + GIV-C bounding strategy may fail, just as all other methods fail. As a practical matter, therefore, accurate estimates of the effects of education on wages require strategies for identifying and reducing the impact of environmental endogeneity. Therefore, the bounds reported here only reflect the extent of confounds due to the linear effects of common genetic variants in the returns to schooling.

In addition to the two GIV models, we consider a baseline OLS model excluding PGI, as well as a naïve model, where the PGI is included as a control variable without accounting for attenuation bias due to estimation errors in the GWAS. Note that we do not use the MTAG income PGI here.

Table 5 presents our results. The estimate with the baseline controls for the pooled sample (Panel A, column 1) suggests that one additional year of schooling is associated with an average increase in hourly wages of $11 \%(95 \%$ CI: 9.7-12.4), which is slightly higher than earlier estimates from cross-sectional OLS in other US samples (Card 1999; Harmon, Oosterbeek, and Walker 2003; J. J. Heckman, Lochner, and Todd 2006). However, the HRS is a sample of elderly individuals who are approaching or who already are at the end of their professional careers, which could contribute to the slightly higher returns to schooling we find here. Previous attempts to uncover causal estimates of the returns to schooling have shown that the cross-sectional OLS estimates tend to be lower than instrumental variable based approaches (Harmon, Oosterbeek, and Walker 2003). The second column shows the results when the PGI is naïvely controlled for, i.e., by simply adding the income PGI as an additional control variable in an OLS regression. The estimated returns to schooling decreases slightly to $10.7 \%$ (95\% CI: 9.4-12.1) for each additional year of schooling. Notably, a one-standard-deviation increase in the PGI in this model is associated with 3\% higher hourly wages even after adjusting for educational attainment (95\% CI: 1.3-4.8). Due to the measurement error in the PGI for income, this is a downward biased, lower-bound estimate of the relevance of genetic effects on income after controlling for education.

The coefficient estimates for the returns to schooling decrease marginally $(\sim 0.5$ percentage points) after including controls for parental education, which is a proxy for both genetic and environmental advantages that parents pass on to their children. Interestingly, the coefficient 
estimates of income PGI are also slightly lower in models that include these controls $(\sim 0.2$ percentage points), possibly because parental education captures some of the indirect genetic effects that work via favorable environmental conditions that highly educated parents tend to provide for their children (Kong et al. 2018).

Columns 3 and 4 in Panel 1 show the estimates that correct the measurement error in the income PGI with GIV-C and GIV-U regression, providing upper and lower bounds, respectively, of the coefficients for educational attainment conditional on observed genetic confounds. Our results suggest that the average return for an additional year of schooling is between $10.3 \%$ and $10.4 \%$ even after adjusting for the now observed linear common genetic confounds (95\% CI: 8.7-11.8; 8.8-12.0). Furthermore, GIV yields substantially larger estimates of the genetic effects, with a one-standard-deviation increase in the PGI being associated with $8.4 \%$ to $15.8 \%$ higher hourly wages after adjusting for educational attainment (95\% CI: 2.9 $13.9 ; 5.2-26.4)$. These results decrease slightly when controls for parental education are included (7.9\% and $14.8 \%)$.

Our sex-specific estimation results suggest that the returns to schooling are substantially higher for women than for men in the HRS, which is in line with previous studies. The gender differential in returns to schooling has been well documented and has previously been attributed to differences in discrimination, tastes, and circumstances of highly educated women compared to less educated women (Dougherty 2005). In particular, the baseline OLS model estimates an average return of 7.8\% (95\% CI: 5.7-10) for an additional year for schooling for men (Panel B, column 1) but almost twice as much for women (13.3\%; 95\% CI: 11.5-15.1) (Panel C, column 1). The estimated returns decrease by almost the same small magnitude for both men and women when we adjust for potential genetic confounds (Panels B and C, columns $2-4)$. Furthermore, having a college degree seems to yield an additional 10\% (95\% CI: 1-18) income advantage for women over and above the $12 \%$ (95\% CI: 9-15) higher hourly wages for an additional year of schooling in the GIV models (Panel C, column 3-4).

As a robustness check, the same analyses in the HRS are repeated with 3-year moving averages of wages, which reduces measurement error and transitory variance in the wage distribution. As reported in Table A10, the overall results are largely similar to the original results, while some statistical precision is lost due to a smaller sample size.

Our results are comparable to the results of studies that used differences between monozygotic twins to estimate the returns to schooling. For instance, Ashenfelter and Rouse (1998) report that including family fixed effects reduces the returns to schooling from $11 \%$ (95\% CI: $0.09-0.13)$ to $7 \%$ (95\% CI: 0.03-0.11) in the Princeton Twins survey data. Similarly, 
Behrman et al. (1994) show that the returns to schooling decreases from 7\% (95\% CI: 0.070.07 ) to $3.5 \%$ (95\% CI: $0.03-0.04$ ) in the National Academy of Science-National Research Council Twins and the Minnesota Twin Registry. Although some of these estimates are noisy, controlling for family fixed-effects seems to reduce the returns to schooling more sharply in MZ-twin designs than in our approach. This is not surprising given that our approach controls only for currently observed linear genetic confounding effects and parental education as a measure of family background, whereas the twin approach entirely eliminates the bias arising from all family-specific environments and all linear genetic confounds.

Oster (2019) notes that coefficient stability alone cannot provide evidence against omitted variable bias - it does so only if the additional controls are sufficiently important in explaining the outcome variation. There is only a marginal increase in $R^{2}$ when we use the naïve control strategy. However, while we cannot obtain $R^{2}$ from IV regression directly, the substantially larger coefficient estimate of the PGI in GIV regressions may imply a nonnegligible change in $R^{2}$ when the measurement error in the PGI is adequately corrected for.

In summary, controlling for now observable confounds from linear effects of common genetic variants slightly decreases the estimated returns to schooling, but not by more than 0.8 percentage points. At the same time, the estimated relationship between genetic predisposition and realized income remains substantial even after we control for educational attainment. Even in regressions that explicitly control for one's own and one's parents' education, a onestandard-deviation increase in the PGI is associated with an $8-15 \%$ higher average wage in the pooled GIV-C and GIV-U models.

\section{Discussion}

Conceptually, genetic endowments are a form of luck — they are one-time, irreversible, exogenously given, individual-specific endowments that result from the natural experiment of meiosis that randomly mixes the genotypes of one's biological parents. We have shown here that genetic fortune for high income, in the form of random genetic differences between siblings, contributes to inequalities throughout the life course, influencing the education people attain, which occupations they pursue, how much they earn, the quality of the neighborhoods they live in, and the type of health outcomes they will tend to experience in late adulthood. Our results illustrate how tightly health, skills, work, achievements, and genetic luck are coupled: the idea that human agency in the form of choices and effort could be neatly separated from luck is unsubstantiated in light of the life-long consequences of the genetic lottery that 
influence behavior and achievements. The inequalities due to genetic luck that we showed here clearly violate the principle of equal opportunity. They also raise questions about how much credit and responsibilities society can or should attribute to individual's socio-economic and health-related outcomes in life (Roemer 1998; Rawls 1999). If inequalities partly result from a genetic lottery, the case in favor of a social contract that provides insurance against unfavorable outcomes is strong (Alesina and La Ferrara 2005; Alesina, Stantcheva, and Teso 2018; Cappelen et al. 2013; Gromet, Hartson, and Sherman 2015).

Specifically, our results show that the positive relationship between SES and health (Stringhini et al. 2017a; Wilkinson and Marmot 2003; Chetty et al. 2016) is due partly to family-specific genetic and environmental endowments that affect both factors. Furthermore, siblings who "won" the genetic lottery for income are more likely to have favorable health outcomes later in life (e.g., lower BMI), but this genetic advantage is partly mediated by obtaining a college degree. Although our study design does not allow us to identify the causal effect of education on health, our results strongly suggest that high educational attainment and its accompaniments tend to bring about a lifestyle that has health benefits. Furthermore, we have shown that genetic fortune for income also causes differences in educational attainment. However, even when we control for the currently observed genetic confounds, the positive relationship between income and educational attainment remains strong, with an average of 8$11 \%$ higher hourly wages for each additional year of schooling. These results illustrate that the causal pathways from genes to behavior, achievements, and health involve environmental and behavioral pathways that can be intervened upon, such as education. Thus, genes contribute to inequality, but this does not imply biological determinism or an irrelevance of policy.

Genetic predispositions, such as those we studied here, have relevance for all branches of economics that are concerned with differences between individuals (Harden and Koellinger 2020). The rapidly growing availability of genetic data and improvements in computing power and statistical methods now allow us to investigate links between genetic and environmental factors, human behaviour, and economic outcomes directly. This new type of data now permits economists to use genetically-informed study designs that enrich our empirical toolbox and that allow us to ask new questions and to gain new insights on core questions of our discipline. Our results here are illustrations of this. 


\section{References}

Abdellaoui, Abdel, David Hugh-Jones, Loic Yengo, Kathryn E. Kemper, Michel G. Nivard, Laura Veul, Yan Holtz, et al. 2019. "Genetic Correlates of Social Stratification in Great Britain." Nature Human Behaviour 3 (12): 1332-42.

Adler, N. E., T. Boyce, M. A. Chesney, S. Cohen, S. Folkman, R. L. Kahn, and S. L. Syme 1994. "Socioeconomic Status and Health. The Challenge of the Gradient." American Psychology 49 (1): 15-24.

Alesina, Alberto, and Eliana La Ferrara. 2005. "Preferences for Redistribution in the Land of Opportunities." Journal of Public Economics 89 (5): 897-931.

Alesina, Alberto, Stefanie Stantcheva, and Edoardo Teso. 2018. "Intergenerational Mobility and Preferences for Redistribution." The American Economic Review 108 (2): 521-54.

Almås, Ingvild, Alexander W. Cappelen, Erik Ø. Sørensen, and Bertil Tungodden. 2010. "Fairness and the Development of Inequality Acceptance." Science 328 (5982): 1176-78.

Anderson, Michael L. 2008. "Multiple Inference and Gender Differences in the Effects of Early Intervention: A Reevaluation of the Abecedarian, Perry Preschool, and Early Training Projects." Journal of the American Statistical Association. https://doi.org/10.1198/016214508000000841.

Arcidiacono, Peter, Patrick Bayer, and Aurel Hizmo. 2010. "Beyond Signaling and Human Capital: Education and the Revelation of Ability." American Economic Journal. Applied Economics 2 (4): 76-104.

Ashenfelter, Orley, and Cecilia Rouse. 1998. "Income, Schooling, and Ability: Evidence from a New Sample of Identical Twins." Quarterly Journal of Economics 113: 253-84.

Auton, Adam, Goncalo R. Abecasis, David M. Altshuler, Richard M. Durbin, David R. Bentley, Aravinda Chakravarti, Andrew G. Clark, et al. 2015. "Genetic Variance Estimation with Imputed Variants Finds Negligible Missing Heritability for Human Height and Body Mass Index." Nature Genetics 526 (7571): 1114-20.

Barcellos, Silvia H., Leandro S. Carvalho, and Patrick Turley. 2018. "Education Can Reduce Health Differences Related to Genetic Risk of Obesity." Proceedings of the National Academy of Sciences of the United States of America 115 (42): E9765-72.

Barth, Daniel, Nicholas W. Papageorge, and Kevin Thom. 2020. "Genetic Endowments and Wealth Inequality." The Journal of Political Economy, no. 24642. https://doi.org/10.3386/w24642.

Becker, Gary S., Scott Duke Kominers, Kevin M. Murphy, and Jörg L. Spenkuch. 2018. "A Theory of Intergenerational Mobility." The Journal of Political Economy 126 (S1): S7-25.

Behrman, Jere R., Mark R. Rosenzweig, and Paul Taubman. 1994. "Endowments and the Allocation of Schooling in the Family and in the Marriage Market: The Twins Experiment." Journal of Political Economy. https://doi.org/10.1086/261966.

Belsky, Daniel W., Benjamin W. Domingue, Robbee Wedow, Louise Arseneault, Jason D. Boardman, Avshalom Caspi, Dalton Conley, et al. 2018. "Genetic Analysis of Social-Class Mobility in Five Longitudinal Studies." Proceedings of the National Academy of Sciences of the United States of America 115 (31): E7275-84.

Benjamin, D. J., D. Cesarini, C. F. Chabris, E. L. Glaeser, D. I. Laibson, V. Gudnason, T. B. Harris, et al. 2012. "The Promises and Pitfalls of Genoeconomics." Annual Review of Economics 4 (1): $627-62$.

Bowles, Samuel, and Herbert Gintis. 2002. "The Inheritance of Inequality." The Journal of Economic Perspectives: A Journal of the American Economic Association 16 (3): 3-30.

Bulik-Sullivan, Brendan, Hilary K. Finucane, Verneri Anttila, Alexander Gusev, Felix R. Day, Reprogen Consortium, Psychiatric Genomics Consortium, et al. 2015. "An Atlas of Genetic Correlations across Human Diseases and Traits.” Nature Genetics 47 (11): 1236-41.

Cappelen, Alexander W., James Konow, Erik Ø. Sørensen, and Bertil Tungodden. 2013. "Just Luck: An Experimental Study of Risk-Taking and Fairness." The American Economic Review 103 (4): $1398-1413$.

Card, David. 1999. "The Causal Effect of Education on Earnings." Handbook of Labor Economics. https://doi.org/10.1016/s1573-4463(99)03011-4.

Cesarini, David, Magnus Johannesson, Paul Lichtenstein, Örjan Sandewall, and Björn Wallace. 2010. 
“Genetic Variation in Financial Decision-Making." The Journal of Finance 65 (5): 1725-54.

Cesarini, D., C. T. Dawes, M. Johannesson, P. Lichtenstein, and B. Wallace. 2009. "Genetic Variation in Preferences for Giving and Risk Taking." The Quarterly Journal of Economics 124 (2): 80942.

Chabris, Christopher F., James J. Lee, David Cesarini, Daniel J. Benjamin, and David I. Laibson. 2015. "The Fourth Law of Behavior Genetics." Current Directions in Psychological Science 24 (4): 304-12.

Chetty, Raj, and Nathaniel Hendren. 2018. "The Impacts of Neighborhoods on Intergenerational Mobility II: County-Level Estimates.” The Quarterly Journal of Economics 133 (3): 1163-1228.

Chetty, Raj, Michael Stepner, Sarah Abraham, Shelby Lin, Benjamin Scuderi, Nicholas Turner, Augustin Bergeron, and David Cutler. 2016. "The Association between Income and Life Expectancy in the United States, 2001-2014." JAMA: The Journal of the American Medical Association 315 (16): 1750.

Clark, Andrew E., and Conchita D'Ambrosio. 2015. "Chapter 13 - Attitudes to Income Inequality: Experimental and Survey Evidence." In Handbook of Income Distribution, edited by Anthony B. Atkinson and François Bourguignon, 2:1147-1208. Elsevier.

Comfort, Nathaniel. 2018. "Genetic Determinism Rides Again." Nature 561 (7724): 461-63.

Corak, Miles. 2013. "Income Inequality, Equality of Opportunity, and Intergenerational Mobility." The Journal of Economic Perspectives: A Journal of the American Economic Association 27 (3): 79-102.

Daetwyler, H. D., B. Villanueva, and J. A. Woolliams. 2008. "Accuracy of Predicting the Genetic Risk of Disease Using a Genome-Wide Approach.” PloS One 3 (10): e3395.

Davies, Neil M., Laurence J. Howe, Ben Brumpton, Alexandra Havdahl, David M. Evans, and George Davey Smith. 2019. "Within Family Mendelian Randomization Studies." Human Molecular Genetics 28 (R2): R170-79.

DiPrete, Thomas A., Casper Burik, and Philipp Koellinger. 2018. "Genetic Instrumental Variable Regression: Explaining Socioeconomic and Health Outcomes in Nonexperimental Data." Proceedings of the National Academy of Sciences of the United States of America 115 (22): E4970-79.

Dougherty, Christopher. 2005. "Why Are the Returns to Schooling Higher for Women than for Men?" Journal of Human Resources. https://doi.org/10.3368/jhr.xl.4.969.

Durlauf, Steven N., and Ananth Seshadri. 2018. "Understanding the Great Gatsby Curve." NBER Macroeconomics Annual 32 (April): 333-93.

Editors, Nature. 2013. "Dangerous Work." Nature 502 (7469): 5-6.

Falconer, Douglas S., and Trudy F. C. Mackay. 2009. Introduction to Quantitative Genetics. Pearson.

Felson, Jacob. 2014. "What Can We Learn from Twin Studies? A Comprehensive Evaluation of the Equal Environments Assumption.” Social Science Research 43 (January): 184-99.

Fry, Anna, Thomas J. Littlejohns, Cathie Sudlow, Nicola Doherty, Ligia Adamska, Tim Sprosen, Rory Collins, and Naomi E. Allen. 2017. "Comparison of Sociodemographic and Health-Related Characteristics of UK Biobank Participants With Those of the General Population." American Journal of Epidemiology 186 (9): 1026-34.

Global Burden of Disease Obesity Collaborators, Ashkan Afshin, Mohammad H. Forouzanfar, Marissa B. Reitsma, Patrick Sur, Kara Estep, Alex Lee, et al. 2017. "Health Effects of Overweight and Obesity in 195 Countries over 25 Years." The New England Journal of Medicine 377 (1): 13-27.

Goldberger, Arthur S. 1978. "The Genetic Determination of Income: Comment." The American Economic Review 68 (5): 960-69.

- 1979. "Heritability." Economica 46 (184): 327-47.

Griliches, Zvi. 1977. "Estimating the Returns to Schooling: Some Econometric Problems." Econometrica: Journal of the Econometric Society 45 (1): 1-22.

Gromet, Dena M., Kimberly A. Hartson, and David K. Sherman. 2015. "The Politics of Luck: Political Ideology and the Perceived Relationship between Luck and Success." Journal of Experimental Social Psychology 59 (July): 40-46.

Grossman, Michael. 2000. “The Human Capital Model.” In Handbook of Health Economics, 1:347408. Elsevier. 
2006. "Chapter 10 Education and Nonmarket Outcomes." In Handbook of the Economics of Education, edited by E. Hanushek and F. Welch, 1:577-633. Elsevier.

Hamer, Dean H., and L. Sirota. 2000. "Beware the Chopsticks Gene." Molecular Psychiatry 5 (1): $11-13$.

Harden, K. P., and P. D. Koellinger. 2020. "Using Genetics for Social Science.” Nature Human Behaviour. https://doi.org/10.1038/s41562-020-0862-5.

Harmon, Colm, Hessel Oosterbeek, and Ian Walker. 2003. "The Returns to Education: Microeconomics.” Journal of Economic Surveys. https://doi.org/10.1111/1467-6419.00191.

Haworth, Simon, Ruth Mitchell, Laura Corbin, Kaitlin H. Wade, Tom Dudding, Ashley BuduAggrey, David Carslake, et al. 2019. "Apparent Latent Structure within the UK Biobank Sample Has Implications for Epidemiological Analysis." Nature Communications 10 (1): 333.

Heckman, James J., Lance J. Lochner, and Petra E. Todd. 2006. "Chapter 7: Earnings Functions, Rates of Return and Treatment Effects: The Mincer Equation and beyond." In Handbook of the Economics of Education, Volume 1, edited by E. Hanushek and F. Welch, 1:307-458. Elsevier.

Heckman, James, and Rodrigo Pinto. 2015. "Econometric Mediation Analyses: Identifying the Sources of Treatment Effects from Experimentally Estimated Production Technologies with Unmeasured and Mismeasured Inputs." Econometric Reviews 34 (1-2): 6-31.

Henrich, Joseph, Steven J. Heine, and Ara Norenzayan. 2010. "The Weirdest People in the World?" The Behavioral and Brain Sciences 33 (2-3): 61-83; discussion 83-135.

Hill, W. David, Neil M. Davies, Stuart J. Ritchie, Nathan G. Skene, Julien Bryois, Steven Bell, Emanuele Di Angelantonio, et al. 2019. "Genome-Wide Analysis Identifies Molecular Systems and 149 Genetic Loci Associated with Income.” Nature Communications 10 (1): 5741.

Hill, W. David, Saskia P. Hagenaars, Riccardo E. Marioni, Sarah E. Harris, David C. M. Liewald, Gail Davies, Aysu Okbay, Andrew M. McIntosh, Catharine R. Gale, and Ian J. Deary. 2016. "Molecular Genetic Contributions to Social Deprivation and Household Income in UK Biobank." Current Biology: CB 26 (22): 3083-89.

Holm, Sture. 1979. “A Simple Sequentially Rejective Multiple Test Procedure.” Scandinavian Journal of Statistics, Theory and Applications 6 (2): 65-70.

Huxley, R., S. Mendis, E. Zheleznyakov, S. Reddy, and J. Chan. 2010. "Body Mass Index, Waist Circumference and Waist:hip Ratio as Predictors of Cardiovascular Risk--a Review of the Literature." European Journal of Clinical Nutrition 64 (1): 16-22.

Imai, Kosuke, Luke Keele, and Teppei Yamamoto. 2010. "Identification, Inference and Sensitivity Analysis for Causal Mediation Effects." Statistical Science. https://doi.org/10.1214/10-sts321.

Jäntti, Markus, and Stephen P. Jenkins. 2015. "Chapter 10 - Income Mobility." In Handbook of Income Distribution, edited by Anthony B. Atkinson and François Bourguignon, 2:807-935. Elsevier.

Jencks, Christopher. 1972. Inequality: A Reassessment of the Effect of Family and Schooling in America. New York: Basic Books, Inc.

_. 1980. "Heredity, Environment, and Public Policy Reconsidered." American Sociological Review 45 (5): 723-36.

Karlsson Linnér, Richard, Pietro Biroli, Edward Kong, S. Fleur W. Meddens, Robbee Wedow, Mark Alan Fontana, Maël Lebreton, et al. 2019. "Genome-Wide Association Analyses of Risk Tolerance and Risky Behaviors in over 1 Million Individuals Identify Hundreds of Loci and Shared Genetic Influences." Nature Genetics 51: 245-57.

Kawachi, Ichiro, Nancy E. Adler, and William H. Dow. 2010. "Money, Schooling, and Health: Mechanisms and Causal Evidence." Annals of the New York Academy of Sciences 1186 (February): 56-68.

Kevles, Daniel J. 1995. In the Name of Eugenics: Genetics and the Uses of Human Heredity. Harvard University Press.

Kong, Augustine, Gudmar Thorleifsson, Michael L. Frigge, Bjarni J. Vilhjalmsson, Alexander I. Young, Thorgeir E. Thorgeirsson, Stefania Benonisdottir, et al. 2018. "The Nature of Nurture: Effects of Parental Genotypes." Science 359 (6374): 424-28.

Kuznets, Simon. 1955. "Economic Growth and Income Inequality." The American Economic Review 45 (1): 1-28.

Ladd-Taylor, Molly. 2020. Fixing the Poor. Johns Hopkins University Press. 
Lee, James J., Robbee Wedow, Aysu Okbay, Edward Kong, Omeed Maghzian, Meghan Zacher, Tuan Anh Nguyen-Viet, et al. 2018. "Gene Discovery and Polygenic Prediction from a Genome-Wide Association Study of Educational Attainment in 1.1 Million Individuals." Nature Genetics 50 (8): 1112-21.

Lerner, R. M. 2006. “Another Nine-Inch Nail for Behavioral Genetics!” Human Development 49 (6): $336-42$.

Lo, Min-Tzu, David A. Hinds, Joyce Y. Tung, Carol Franz, Chun-Chieh Fan, Yunpeng Wang, Olav B. Smeland, et al. 2016. "Genome-Wide Analyses for Personality Traits Identify Six Genomic Loci and Show Correlations with Psychiatric Disorders." Nature Genetics 49 (1): 152-56.

Manski, Charles F. 2011. "Genes, Eyeglasses, and Social Policy.” The Journal of Economic Perspectives: A Journal of the American Economic Association 25 (4): 83-94.

Michael, Spence. 1973. "Job Market Signaling." The Quarterly Journal of Economics 87 (3): 355-74.

Mills, Melinda C., Nicola Barban, and Felix C. Tropf. 2020. An Introduction to Statistical Genetic Data Analysis. Cambridge, MA: The MIT Press.

Mincer, Jacob. 1958. "Investment in Human Capital and Personal Income Distribution." The Journal of Political Economy 66 (4): 281-302.

Nicolaou, Nicos, and Scott Shane. 2010. "Entrepreneurship and Occupational Choice: Genetic and Environmental Influences." Journal of Economic Behavior \& Organization 76 (1): 3-14.

Nuffield Council on Bioethics. 2002. "Genetics and Human Behaviour: The Ethical Context." Nuffield Council on Bioethics. http://nuffieldbioethics.org/wpcontent/uploads/2014/07/Genetics-and-human-behaviour.pdf.

O’Donnell, Owen, Eddy Van Doorslaer, and Tom Van Ourti. 2015. "Health and Inequality." Handbook of Income Distribution. https://doi.org/10.1016/b978-0-444-59429-7.00018-2.

Okbay, Aysu, Jonathan P. Beauchamp, Mark A. Fontana, James J. Lee, Tune H. Pers, Cornelius A. Rietveld, Patrick Turley, et al. 2016. "Genome-Wide Association Study Identifies 74 Loci Associated with Educational Attainment." Nature 533: 539-42.

Oster, Emily. 2019. "Unobservable Selection and Coefficient Stability: Theory and Evidence." Journal of Business \& Economic Statistics. https://doi.org/10.1080/07350015.2016.1227711.

Papageorge, Nicholas W., and Kevin Thom. 2019. "Genes, Education, and Labor Market Outcomes: Evidence from the Health and Retirement Study." Journal of the European Economic Association, December. https://doi.org/10.1093/jeea/jvz072.

Piketty, Thomas, and Emmanuel Saez. 2003. "Income Inequality in the United States, 1913-1998." The Quarterly Journal of Economics 118 (1): 1-41.

Piotrowska, Patrycja J., Christopher B. Stride, Simone E. Croft, and Richard Rowe. 2015. "Socioeconomic Status and Antisocial Behaviour among Children and Adolescents: A Systematic Review and Meta-Analysis." Clinical Psychology Review 35 (February): 47-55.

Plomin, Robert, John DeFries, Valerie Knopik, and Jenae Neiderhiser. 2012. Behavioral Genetics. Vol. 6. Worth Publishers.

Polderman, Tinca J. C., Beben Benyamin, Christiaan A. de Leeuw, Patrick F. Sullivan, Arjen van Bochoven, Peter M. Visscher, and Danielle Posthuma. 2015. "Meta-Analysis of the Heritability of Human Traits Based on Fifty Years of Twin Studies." Nature Genetics 47: 702-9.

Price, Alkes L., Nick J. Patterson, Robert M. Plenge, Michael E. Weinblatt, Nancy A. Shadick, and David Reich. 2006. "Principal Components Analysis Corrects for Stratification in Genome-Wide Association Studies." Nature Genetics 38 (8): 904-9.

Purcell, Shaun. 2002. "Variance Components Models for Gene-environment Interaction in Twin Analysis." Twin Research and Human Genetics: The Official Journal of the International Society for Twin Studies 5 (6): 554-71.

Rawls, John. 1999. A Theory of Justice. Belknap Press of Harvard University Press.

Rietveld, Cornelius A., Sarah E. Medland, Jaime Derringer, Jian Yang, Tõnu Esko, Nicolas W. Martin, Harm-Jan Westra, et al. 2013. "GWAS of 126,559 Individuals Identifies Genetic Variants Associated with Educational Attainment." Science 340 (6139): 1467-71.

Roberts, Dorothy. 2015. "Can Research on the Genetics of Intelligence Be 'socially Neutral'?" The Hastings Center Report 45 (1): S50-53.

Roemer, John E. 1998. Equality of Opportunity. Harvard University Press.

Roemer, John E., and Alain Trannoy. 2015. "Chapter 4 - Equality of Opportunity.” In Handbook of 
Income Distribution, edited by Anthony B. Atkinson and François Bourguignon, 2:217-300. Elsevier.

- 2016. "Equality of Opportunity: Theory and Measurement." Journal of Economic Literature 54 (4): 1288-1332.

Roine, Jesper, and Daniel Waldenström. 2015. "Chapter 7 - Long-Run Trends in the Distribution of Income and Wealth." In Handbook of Income Distribution, edited by Anthony B. Atkinson and François Bourguignon, 2:469-592. Elsevier.

Rowe, David C., Wendy J. Vesterdal, and Joseph L. Rodgers. 1998. "Herrnstein's Syllogism: Genetic and Shared Environmental Influences on IQ, Education, and Income." Intelligence 26 (4): $405-$ 23.

Sacerdote, Bruce. 2002. "The Nature and Nurture of Economic Outcomes." The American Economic Review 92 (2): 344-48.

Sacks, Daniel W., Betsey Stevenson, and Justin Wolfers. 2012. "The New Stylized Facts about Income and Subjective Well-Being." Emotion 12 (6): 1181-87.

Selzam, Saskia, Stuart J. Ritchie, Jean-Baptiste Pingault, Chandra A. Reynolds, Paul F. O'Reilly, and Robert Plomin. 2019. "Comparing Within- and Between-Family Polygenic Score Prediction." American Journal of Human Genetics 105 (2): 351-63.

Sonnega, A., J. D. Faul, M. B. Ofstedal, K. M. Langa, J. W. Phillips, and D. R. Weir. 2014. "Cohort Profile: The Health and Retirement Study (HRS)." International Journal of Epidemiology. https://doi.org/10.1093/ije/dyu067.

Srikanthan, Preethi, Teresa E. Seeman, and Arun S. Karlamangla. 2009. "Waist-Hip-Ratio as a Predictor of All-Cause Mortality in High-Functioning Older Adults." Annals of Epidemiology 19 (10): 724-31.

Stevenson, Betsey, and Justin Wolfers. 2013. "Subjective Well-Being and Income: Is There Any Evidence of Satiation?" The American Economic Review 103 (3): 598-604.

Stringhini, Silvia, Cristian Carmeli, Markus Jokela, Mauricio Avendaño, Peter Muennig, Florence Guida, Fulvio Ricceri, et al. 2017a. "Socioeconomic Status and the $25 \times 25$ Risk Factors as Determinants of Premature Mortality: A Multicohort Study and Meta-Analysis of 1.7 Million Men and Women." The Lancet 389 (10075): 1229-37.

- 2017b. "Socioeconomic Status and the $25 \times 25$ Risk Factors as Determinants of Premature Mortality: A Multicohort Study and Meta-Analysis of 11textperiodcentered 7 Million Men and Women." The Lancet 389 (10075): 1229-37.

Sudlow, Cathie, John Gallacher, Naomi Allen, Valerie Beral, Paul Burton, John Danesh, Paul Downey, et al. 2015. "UK Biobank: An Open Access Resource for Identifying the Causes of a Wide Range of Complex Diseases of Middle and Old Age." PLoS Medicine 12 (3): e1001779.

Tabery, James. 2015. "Why Is Studying the Genetics of Intelligence so Controversial?" The Hastings Center Report 45 (5): S9-14.

Taubman, Paul. 1976. "The Determinants of Earnings: Genetics, Family, and Other Environments: A Study of White Male Twins." The American Economic Review 66 (5): 858-70.

Turkheimer, Eric. 2000. "Three Laws of Behavior Genetics and What They Mean." Current Directions in Psychological Science 9 (5): 160-64.

Turley, Patrick, Raymond K. Walters, Omeed Maghzian, Aysu Okbay, James J. Lee, Mark Alan Fontana, Tuan Anh Nguyen-Viet, et al. 2018. "Multi-Trait Analysis of Genome-Wide Association Summary Statistics Using MTAG." Nature Genetics 50 (2): 229-37.

Vilhjálmsson, Bjarni J., Jian Yang, Hilary K. Finucane, Alexander Gusev, Sara Lindström, Stephan Ripke, Giulio Genovese, et al. 2015. "Modeling Linkage Disequilibrium Increases Accuracy of Polygenic Risk Scores." American Journal of Human Genetics 97 (4): 576-92.

Visscher, Peter M., William G. Hill, and Naomi R. Wray. 2008. "Heritability in the Genomics Era-Concepts and Misconceptions." Nature Reviews. Genetics 9 (4): 255-66.

Vlaming, Ronald de, Aysu Okbay, Cornelius A. Rietveld, Magnus Johannesson, Patrik K. E. Magnusson, André G. Uitterlinden, Frank J. A. van Rooij, et al. 2017. "Meta-GWAS Accuracy and Power (MetaGAP) Calculator Shows That Hiding Heritability Is Partially due to Imperfect Genetic Correlations across Studies." PLoS Genetics 13 (1). https://doi.org/10.1371/journal.pgen.1006495.

Weir, David. 2013. "Quality Control Report for Genotypic Data.” September 4, 2013. 
http://hrsonline.isr.umich.edu/sitedocs/genetics/HRS2_qc_report_SEPT2013.pdf.

Wilkinson, Richard G., and Michael Marmot. 2003. Social Determinants of Health: The Solid Facts. World Health Organization.

World Health Organization. 2019. "International Classification of Diseases (ICD)." International Classification of Diseases (ICD). World Health Organization. October 11, 2019. https://www.who.int/classifications/icd/en/.

Young, Alexander I., Stefania Benonisdottir, Molly Przeworski, and Augustine Kong. 2019. "Deconstructing the Sources of Genotype-Phenotype Associations in Humans." Science 365 (6460): 1396-1400.

Young, Alexander I., Michael L. Frigge, Daniel F. Gudbjartsson, Gudmar Thorleifsson, Gyda Bjornsdottir, Patrick Sulem, Gisli Masson, Unnur Thorsteinsdottir, Kari Stefansson, and Augustine Kong. 2018. "Relatedness Disequilibrium Regression Estimates Heritability without Environmental Bias.” Nature Genetics 50 (9): 1304-10.

Zax, Jeffrey S., and Daniel I. Rees. 2002. "IQ, Academic Performance, Environment, and Earnings." The Review of Economics and Statistics 84 (4): 600-616.

Zimmer, Carl. 2018. She Has Her Mother's Laugh The Powers, Perversions, and Potential of Heredity. Dutton.

Zuk, Or, Eliana Hechter, Shamil R. Sunyaev, and Eric S. Lander. 2012. "The Mystery of Missing Heritability: Genetic Interactions Create Phantom Heritability." Proceedings of the National Academy of Sciences of the United States of America 109 (4): 1193-98. 
Table 1. Association between socioeconomic status (SES) measures and health outcomes in the UK Biobank

\begin{tabular}{|c|c|c|c|c|}
\hline & \multicolumn{2}{|c|}{ log occupational wage per hour } & \multicolumn{2}{|c|}{ College education } \\
\hline & OLS & OLS-FE & OLS & OLS-FE \\
\hline \multicolumn{5}{|l|}{ Dependent variable } \\
\hline summary index & $-0.126 * * *$ & -0.049 & $-0.112 * * *$ & $-0.046 * * *$ \\
\hline$(\mathrm{N}=13,862 \mid 26,550)$ & $(0.009)$ & $(0.018)$ & $(0.005)$ & $(0.011)$ \\
\hline waist-to-hip ratio & $-0.019 * * *$ & -0.007 & $-0.017 * * *$ & $-0.007 * *$ \\
\hline$(\mathrm{N}=17,658 \mid 35,028)$ & $(0.002)$ & $(0.003)$ & $(0.001)$ & $(0.002)$ \\
\hline BMI & $-1.248 * * *$ & -0.103 & $-1.298 * * *$ & $-0.415 * *$ \\
\hline$(\mathrm{N}=17,644 \mid 34,968)$ & $(0.108)$ & $(0.202)$ & $(0.055)$ & $(0.111)$ \\
\hline blood pressure & $-2.531 * * *$ & -0.788 & $-1.885^{* * *}$ & $-1.185^{*}$ \\
\hline$(\mathrm{N}=15,818 \mid 31,372)$ & $(0.326)$ & $(0.663)$ & $(0.171)$ & $(0.371)$ \\
\hline lung function & $0.279 * * *$ & 0.097 & $0.188 * * *$ & $0.082 * *$ \\
\hline$(\mathrm{N}=15,506 \mid 29,844)$ & $(0.019)$ & $(0.040)$ & $(0.010)$ & $(0.022)$ \\
\hline ever hospitalized & $-0.061 * * *$ & -0.019 & $-0.047 * * *$ & -0.007 \\
\hline$(\mathrm{N}=17,692 \mid 35,132)$ & $(0.009)$ & $(0.021)$ & $(0.005)$ & $(0.011)$ \\
\hline ever diagnosed with cancer & 0.006 & -0.008 & 0.002 & 0.004 \\
\hline$(\mathrm{N}=17,692 \mid 35,132)$ & $(0.008)$ & $(0.018)$ & $(0.004)$ & $(0.011)$ \\
\hline infectious and parasitic diseases & $-0.030 * * *$ & -0.017 & $-0.028 * * *$ & -0.003 \\
\hline$(\mathrm{N}=17,692 \mid 35,132)$ & $(0.006)$ & $(0.014)$ & $(0.003)$ & $(0.008)$ \\
\hline neoplasms & 0.013 & -0.007 & 0.006 & 0.005 \\
\hline$(\mathrm{N}=17,692 \mid 35,132)$ & $(0.008)$ & $(0.017)$ & $(0.004)$ & $(0.010)$ \\
\hline diseases of blood organs and immune system & $-0.028 * *$ & -0.006 & $-0.031 * * *$ & -0.009 \\
\hline$(\mathrm{N}=17,692 \mid 35,132)$ & $(0.009)$ & $(0.020)$ & $(0.005)$ & $(0.012)$ \\
\hline endocrine, nutritional, and metabolic diseases & $-0.054 * * *$ & -0.011 & $-0.069 * * *$ & -0.024 \\
\hline$(\mathrm{N}=17,692 \mid 35,132)$ & $(0.008)$ & $(0.018)$ & $(0.004)$ & $(0.011)$ \\
\hline mental, behavioral, nervous system disorders & $-0.071 * * *$ & -0.047 & $-0.059 * * *$ & -0.026 \\
\hline$(\mathrm{N}=17,692 \mid 35,132)$ & $(0.008)$ & $(0.019)$ & $(0.004)$ & $(0.010)$ \\
\hline diseases of the eye and adnexa & -0.006 & -0.009 & $-0.017 * * *$ & -0.019 \\
\hline$(\mathrm{N}=17,692 \mid 35,132)$ & $(0.006)$ & $(0.014)$ & $(0.004)$ & $(0.009)$ \\
\hline
\end{tabular}


Table 1. Association between socioeconomic status (SES) measures and health outcomes in the UK Biobank

\begin{tabular}{|c|c|c|c|c|}
\hline & \multicolumn{2}{|c|}{ log occupational wage per hour } & \multicolumn{2}{|c|}{ College education } \\
\hline & OLS & OLS-FE & OLS & OLS-FE \\
\hline diseases of the circulatory system & $-0.081 * * *$ & -0.017 & $-0.086 * * *$ & $-0.038^{*}$ \\
\hline$(\mathrm{N}=17,692 \mid 35,132)$ & $(0.010)$ & $(0.022)$ & $(0.005)$ & $(0.012)$ \\
\hline diseases of the respiratory system & $-0.051 * * *$ & -0.027 & $-0.047 * * *$ & -0.018 \\
\hline$(\mathrm{N}=17,692 \mid 35,132)$ & $(0.008)$ & $(0.017)$ & $(0.004)$ & $(0.010)$ \\
\hline diseases of the digestive system & $-0.090 * * *$ & -0.026 & $-0.075 * * *$ & -0.010 \\
\hline$(\mathrm{N}=17,692 \mid 35,132)$ & $(0.011)$ & $(0.024)$ & $(0.006)$ & $(0.014)$ \\
\hline diseases of the skin and subcutaneous tissue & -0.014 & -0.011 & $-0.023 * * *$ & -0.018 \\
\hline$(\mathrm{N}=17,692 \mid 35,132)$ & $(0.007)$ & $(0.015)$ & $(0.004)$ & $(0.009)$ \\
\hline diseases of musculoskeletal system and connective tissue & $-0.065 * * *$ & -0.026 & $-0.068 * * *$ & -0.029 \\
\hline$(\mathrm{N}=17,692 \mid 35,132)$ & $(0.010)$ & $(0.022)$ & $(0.005)$ & $(0.012)$ \\
\hline diseases of genitourinary system & $-0.063 * * *$ & -0.014 & $-0.053 * * *$ & -0.017 \\
\hline$(\mathrm{N}=17,692 \mid 35,132)$ & $(0.010)$ & $(0.021)$ & $(0.005)$ & $(0.012)$ \\
\hline symptoms and signs not elsewhere classified & $-0.068 * * *$ & -0.024 & $-0.066 * * *$ & 0.000 \\
\hline$(\mathrm{N}=17,692 \mid 35,132)$ & $(0.011)$ & $(0.024)$ & $(0.006)$ & $(0.013)$ \\
\hline injury, poisoning, and other consequences of external causes & $-0.035 * * *$ & -0.030 & $-0.015 * *$ & -0.002 \\
\hline$(\mathrm{N}=17,692 \mid 35,132)$ & $(0.008)$ & $(0.018)$ & $(0.004)$ & $(0.011)$ \\
\hline external causes of morbidity and mortality & $-0.045^{* * *}$ & -0.043 & $-0.023 * * *$ & -0.007 \\
\hline$(\mathrm{N}=17,692 \mid 35,132)$ & $(0.008)$ & $(0.019)$ & $(0.004)$ & $(0.011)$ \\
\hline other health conditions & $-0.052 * * *$ & -0.025 & $-0.067 * * *$ & -0.026 \\
\hline$(\mathrm{N}=17,692 \mid 35,132)$ & $(0.011)$ & $(0.025)$ & $(0.006)$ & $(0.014)$ \\
\hline
\end{tabular}

Note: The table reports the coefficients from separate regressions of health outcomes on log occupational wages per hour and a dummy variable for college education, with or without family fixed effects (FE). Standard errors clustered by family are reported in parentheses. Significance at family-wise error rate $5 \%(*), 1 \%(* *), 0.1 \%(* * *)$, where multiple hypothesis testing is corrected by Holm's method (Holm, 1979) for each set of analysis. For each outcome, the sample is restricted to sibling pairs for both of whom the outcome is observed. The summary index is a weighted average of all the health outcomes constructed according to Anderson (2008) such that lower values imply a better health. All regressions controlled for a sex dummy, year of birth, year of assessment, and the interaction terms between the sex dummy and all other covariates. Regressions on log hourly wages also included dummies for year and age of observation. 
Table 2. Associations between the polygenic index for income and measures of socioeconomic achievement and health in UK Biobank siblings

OLS

OLS-FE

GIV

GIV-FE

\section{Socioeconomic outcomes}

log hourly wage
$(\mathrm{N}=17,692)$

top household income

$(\mathrm{N}=27,412)$

$\log$ regional income

$(\mathrm{N}=31,692)$

neighborhood score

$(\mathrm{N}=29,166)$

years of education

$(\mathrm{N}=35,132)$

college degree

$(\mathrm{N}=35,132)$

\section{health proxies}

waist-to-hip ratio

$(\mathrm{N}=35,498)$

BMI

$(\mathrm{N}=35,432)$

blood pressure

$(\mathrm{N}=31,770)$

lung function

$(\mathrm{N}=30,240)$

$0.074 * * *$

(0.002)

$0.056^{* * *}$

(0.003)

$0.041 * * *$

(0.001)

$1.523 * * *$

(0.088)

$1.394 * * *$

(0.026)

$0.131 * * *$

(0.002)

$-0.007 * * *$

(0.000)

$-0.563 * * *$

(0.027)

$-0.847 * * *$

(0.078)

$0.055 * * *$

(0.005)
$0.046 * * *$

(0.007)

$0.147 * * *$

(0.008)

$0.084 * *$

$0.034 * * *$

$0.122 * * *$

(0.008)

$(0.007) \quad(0.008) \quad(0.025)$

(0.003)

(0.005)

(0.012)

$\begin{array}{lll}0.643 * & 2.869 * * * & 1.598\end{array}$

(0.203)

(0.284)

(0.694)

$0.771 * * *$

$2.774 * * *$

$1.498 * * *$

(0.066)

(0.095)

(0.237)

$0.069 * * *$

$0.258 * * *$

$0.145^{* * *}$

(0.006)

(0.009)

(0.021)

\begin{abstract}
$-0.004 * *$
\end{abstract}
$-0.015^{* * *}$

$-0.009$

(0.001)

(0.001)

(0.003)

$-0.286 * * * \quad-0.994 * * * \quad-0.497$

(0.086)

(0.223)

(0.063)

$-1.678 * * *$

$-0.795$

$-0.608$

$(0.250)$

$(0.735)$

$0.017 \quad 0.112 * * * \quad 0.052$

$(0.013) \quad(0.015) \quad(0.047)$
reported in parentheses. Significance at family-wise error rate $5 \%(*), 1 \%(* *), 0.1 \%(* * *)$, where multiple testing is controlled using Holm's method (Holm, 1979) for each set of analysis. For each outcome, the sample is restricted to sibling pairs for both of whom the outcome is observed. FE: family fixed effects included. OLS regressions use MTAG PGI for income (i.e. a PGI for income that also takes information from a GWAS on educational attainment into account). GIV regressions use two (non-MTAG) income PGI estimated from two independent samples, where one PGI instruments the other. All analyses included dummy variables for the year of birth, male, and being the younger sibling as well as the first 20 genetic PCs. For economic outcomes, we use age dummies instead of the year of birth and add dummies for the year of survey. For health outcomes we also control for the age dummies instead but not for the year of survey. In every case, we also include the interaction terms between the male dummy and the rest of covariates. 
Table 3. Associations between the polygenic index for income and disease diagnosis outcomes in UK Biobank siblings

\begin{tabular}{|c|c|c|c|c|}
\hline & OLS & OLS-FE & GIV & GIV-FE \\
\hline ever hospitalized & $-0.021 * * *$ & -0.012 & $-0.036^{* * *}$ & -0.028 \\
\hline$(\mathrm{N}=35,602)$ & $(0.002)$ & $(0.006)$ & $(0.006)$ & $(0.020)$ \\
\hline ever diagnosed with cancer & -0.001 & 0.001 & 0.000 & 0.007 \\
\hline$(\mathrm{N}=35,602)$ & $(0.002)$ & $(0.006)$ & $(0.006)$ & $(0.021)$ \\
\hline infectious and parasitic diseases & $-0.013 * * *$ & -0.005 & $-0.026 * * *$ & 0.004 \\
\hline$(\mathrm{N}=35,602)$ & $(0.002)$ & $(0.005)$ & $(0.005)$ & $(0.017)$ \\
\hline neoplasms & 0.000 & 0.001 & 0.002 & 0.007 \\
\hline$(\mathrm{N}=35,602)$ & $(0.002)$ & $(0.006)$ & $(0.006)$ & $(0.021)$ \\
\hline diseases of blood organs and immune system & $-0.012 * * *$ & 0.001 & $-0.024 * *$ & 0.002 \\
\hline$(\mathrm{N}=35,602)$ & $(0.002)$ & $(0.007)$ & $(0.007)$ & $(0.023)$ \\
\hline endocrine, nutritional, and metabolic diseases & $-0.026 * * *$ & -0.011 & $-0.030 * * *$ & 0.007 \\
\hline$(\mathrm{N}=35,602)$ & $(0.002)$ & $(0.006)$ & $(0.007)$ & $(0.022)$ \\
\hline mental, behavioral, nervous system disorders & $-0.027 * * *$ & -0.009 & $-0.048 * * *$ & 0.002 \\
\hline$(\mathrm{N}=35,602)$ & $(0.002)$ & $(0.006)$ & $(0.007)$ & $(0.021)$ \\
\hline diseases of the eye and adnexa & $-0.006 * * *$ & -0.005 & $-0.016^{*}$ & -0.018 \\
\hline$(\mathrm{N}=35,602)$ & $(0.002)$ & $(0.005)$ & $(0.005)$ & $(0.017)$ \\
\hline diseases of the circulatory system & $-0.035 * * *$ & -0.013 & $-0.066^{* * *}$ & -0.035 \\
\hline$(\mathrm{N}=35,602)$ & $(0.003)$ & $(0.007)$ & $(0.008)$ & $(0.025)$ \\
\hline diseases of the respiratory system & $-0.022 * * *$ & -0.010 & $-0.045^{* * *}$ & -0.026 \\
\hline$(\mathrm{N}=35,602)$ & $(0.002)$ & $(0.006)$ & $(0.007)$ & $(0.021)$ \\
\hline diseases of the digestive system & $-0.033 * * *$ & -0.013 & $-0.068 * * *$ & -0.043 \\
\hline$(\mathrm{N}=35,602)$ & $(0.003)$ & $(0.008)$ & $(0.008)$ & $(0.027)$ \\
\hline diseases of the skin and subcutaneous tissue & $-0.008 * * *$ & -0.005 & $-0.013^{*}$ & -0.013 \\
\hline$(\mathrm{N}=35,602)$ & $(0.002)$ & $(0.005)$ & $(0.006)$ & $(0.018)$ \\
\hline diseases of musculoskeletal system and connective tissue & $-0.035 * * *$ & $-0.023 *$ & $-0.065 * * *$ & -0.037 \\
\hline$(\mathrm{N}=35,602)$ & $(0.002)$ & $(0.007)$ & $(0.008)$ & $(0.025)$ \\
\hline diseases of genitourinary system & $-0.022 * * *$ & -0.011 & $-0.051 * * *$ & -0.006 \\
\hline
\end{tabular}


Table 3. Associations between the polygenic index for income and disease diagnosis outcomes in UK Biobank siblings

\begin{tabular}{lcccc}
\hline & OLS & OLS-FE & GIV & GIV-FE \\
\hline ( $=35,602)$ & $(0.002)$ & $(0.007)$ & $(0.008)$ & $(0.024)$ \\
symptoms and signs not elsewhere classified & $-0.033 * * *$ & -0.016 & $-0.064 * * *$ & -0.032 \\
$(\mathrm{~N}=35,602)$ & $(0.003)$ & $(0.008)$ & $(0.008)$ & $(0.027)$ \\
injury, poisoning, and other consequences of external causes & $-0.009 * * *$ & -0.004 & $-0.018 *$ & -0.023 \\
(N=35,602) & $(0.002)$ & $(0.006)$ & $(0.006)$ & $(0.021)$ \\
external causes of morbidity and mortality & $-0.011 * * *$ & -0.004 & $-0.020 *$ & -0.027 \\
(N=35,602) & $(0.002)$ & $(0.006)$ & $(0.007)$ & $(0.022)$ \\
other health conditions & $-0.032 * * *$ & -0.022 & $-0.056 * *$ & -0.039 \\
$(\mathrm{~N}=35,602)$ & $(0.003)$ & $(0.008)$ & $(0.009)$ & $(0.027)$ \\
\hline
\end{tabular}

Note: The table reports the coefficient estimates for the standardized polygenic indice for income (PGI). Standard errors clustered by family are reported in parentheses. Significance at family-wise error rate $5 \%(*), 1 \%(* *), 0.1 \%(* * *)$, where multiple testing is controlled using Holm's method (Holm, 1979) for each set of analysis. For each outcome, the sample is restricted to sibling pairs for both of whom the outcome is observed. FE: family fixed effects included. OLS regressions use MTAG PGI for income (i.e. a PGI for income that also takes information from a GWAS on educational attainment into account). GIV regressions use two (non-MTAG) income PGI estimated from two independent samples, where one PGI instruments the other. All analyses included dummy variables for the year of birth, male, and being the younger sibling as well as the first 20 genetic PCs. For economic outcomes, we use age dummies instead of the year of birth and add dummies for the year of survey. For health outcomes we also control for the age dummies instead but not for the year of survey. In every case, we also include the interaction terms between the male dummy and the rest of covariates. 
Table 4. Decomposition of the genetic lottery effects in the UK Biobank siblings

\begin{tabular}{|c|c|c|c|c|c|}
\hline & estimation & $\begin{array}{c}\text { effect via college } \\
\text { education }\end{array}$ & residual effect & total effect & $\begin{array}{c}\text { effect via college } \\
\text { education } \%\end{array}$ \\
\hline \multirow[t]{2}{*}{$\begin{array}{l}\text { log occupational wage per hour } \\
(\mathrm{N}=17,578)\end{array}$} & OLS & $\begin{array}{l}0.014 * * * \\
(0.002)\end{array}$ & $\begin{array}{l}0.031 * * * \\
(0.006)\end{array}$ & $\begin{array}{l}0.046 * * * \\
(0.007)\end{array}$ & 31.7 \\
\hline & GIV & $\begin{array}{c}0.030 * * * \\
(0.006)\end{array}$ & $\begin{array}{l}0.057^{*} \\
(0.021)\end{array}$ & $\begin{array}{c}0.087 * * * \\
(0.022)\end{array}$ & 34.7 \\
\hline $\begin{array}{l}\text { waist-to-hip ratio } \\
(\mathrm{N}=35,028)\end{array}$ & GIV & $\begin{array}{l}-0.001 * \\
(0.000)\end{array}$ & $\begin{array}{l}-0.008 \\
(0.003)\end{array}$ & $\begin{array}{l}-0.008 \\
(0.003)\end{array}$ & 10.1 \\
\hline $\begin{array}{l}\text { BMI } \\
(\mathrm{N}=34,968)\end{array}$ & OLS & $\begin{array}{l}-0.025 * \\
(0.008)\end{array}$ & $\begin{array}{c}-0.256^{* * *} \\
(0.064)\end{array}$ & $\begin{array}{c}-0.281 * * * \\
(0.064)\end{array}$ & 8.8 \\
\hline \multirow[t]{2}{*}{$\begin{array}{l}\text { blood pressure } \\
(\mathrm{N}=31,372)\end{array}$} & OLS & $\begin{array}{l}-0.077 * \\
(0.027)\end{array}$ & $\begin{array}{l}-0.546^{*} \\
(0.210)\end{array}$ & $\begin{array}{l}-0.622 * \\
(0.209)\end{array}$ & 12.3 \\
\hline & GIV & $\begin{array}{l}-0.159 * \\
(0.060)\end{array}$ & $\begin{array}{l}-0.596 \\
(0.748)\end{array}$ & $\begin{array}{l}-0.755 \\
(0.735)\end{array}$ & 21 \\
\hline \multirow[t]{2}{*}{$\begin{array}{l}\text { lung function } \\
(\mathrm{N}=29,844)\end{array}$} & OLS & $\begin{array}{l}0.005 * * \\
(0.002)\end{array}$ & $\begin{array}{c}0.013 \\
(0.014)\end{array}$ & $\begin{array}{c}0.018 \\
(0.013)\end{array}$ & 29.4 \\
\hline & GIV & $\begin{array}{l}0.011 * \\
(0.004)\end{array}$ & $\begin{array}{c}0.041 \\
(0.048)\end{array}$ & $\begin{array}{c}0.052 \\
(0.047)\end{array}$ & 21.2 \\
\hline
\end{tabular}

Note: $* p<0.05, * * p<0.01, * * * p<0.001$ with Bonferroni correction for testing 5 outcomes. Standard errors clustered by family are reported in parentheses. The standard errors for the indirect effects are computed using the delta method. All regressions used family fixed effects. The table reports decomposition of the genetic lottery effects $i$ for 4 health measures and occupational wages into the effect working via college education and the residual effect. "effect via college education \%" reports the proportion of the effect via college education in the total effect. OLS regressions use MTAG PGI for income. GIV regressions use two income PGI estimated from two independent samples, where one PGI instruments the other. Covariates are the top 20 genetic PCs and dummy variables for the year of birth, male, the age at the time of assessment, and being a younger sibling, as well as the interaction terms between the male dummy and the rest of covariates. For occupational wages, we use age dummies instead of the year of birth and add dummies for the year of survey. For each outcome, the sample is restricted to sibling pairs for both of whom the outcome is observed. 
Table 5. Estimates of income with respect to schooling and genetic factors in the Health and Retirement Study

\begin{tabular}{|c|c|c|c|c|c|c|c|c|c|c|c|c|c|c|c|c|}
\hline \multicolumn{2}{|c|}{ Panel A: Male+Female } & \multicolumn{3}{|c|}{ no PGI } & \multicolumn{4}{|c|}{ naive control } & \multicolumn{4}{|c|}{ GIV-C } & \multicolumn{4}{|c|}{ GIV-U } \\
\hline Educ & $\begin{array}{c}0.1105^{* * *} \\
(0.007)\end{array}$ & $\begin{array}{c}0.1051^{* * *} \\
(0.008)\end{array}$ & $\begin{array}{c}0.0901 * * * \\
(0.010)\end{array}$ & $\begin{array}{c}0.0839^{* * *} \\
(0.011)\end{array}$ & $\begin{array}{c}0.1073 * * * \\
(0.007)\end{array}$ & $\begin{array}{c}0.1023^{* * *} \\
(0.008)\end{array}$ & $\begin{array}{c}0.0878^{* * *} \\
(0.010)\end{array}$ & $\begin{array}{c}0.0820^{* * *} \\
(0.011)\end{array}$ & $\begin{array}{c}0.1040^{* * * *} \\
(0.008)\end{array}$ & $\begin{array}{c}0.0992^{* * *} \\
(0.008)\end{array}$ & $\begin{array}{c}0.0864^{* * *} \\
(0.011)\end{array}$ & $\begin{array}{c}0.0805^{* * *} \\
(0.011)\end{array}$ & $\begin{array}{c}0.1026^{* * *} \\
(0.008)\end{array}$ & $\begin{array}{c}0.0980^{* * *} \\
(0.008)\end{array}$ & $\begin{array}{c}0.0850^{* * *} \\
(0.011)\end{array}$ & $\begin{array}{c}0.0795^{* * *} \\
(0.011)\end{array}$ \\
\hline Educ $\times$ College & & & $\begin{array}{l}0.0602 \\
(0.031)\end{array}$ & $\begin{array}{l}0.0595 \\
(0.031)\end{array}$ & & & $\begin{array}{l}0.0584 \\
(0.031)\end{array}$ & $\begin{array}{l}0.0579 \\
(0.031)\end{array}$ & & & $\begin{array}{l}0.0601 \\
(0.032)\end{array}$ & $\begin{array}{l}0.0593 \\
(0.032)\end{array}$ & & & $\begin{array}{l}0.0538 \\
(0.031)\end{array}$ & $\begin{array}{l}0.0536 \\
(0.031)\end{array}$ \\
\hline Income PGI & & & & & $\begin{array}{c}0.0304 * * * \\
(0.009)\end{array}$ & $\begin{array}{c}0.0287 * * * \\
(0.008)\end{array}$ & $\begin{array}{c}0.0292 * * * \\
(0.008)\end{array}$ & $\begin{array}{c}0.0274 * * \\
(0.008)\end{array}$ & $\begin{array}{c}0.1577 * * \\
(0.054)\end{array}$ & $\begin{array}{c}0.1484 * * \\
(0.053)\end{array}$ & $\begin{array}{c}0.1534 * * \\
(0.054)\end{array}$ & $\begin{array}{c}0.1437 * * \\
(0.053)\end{array}$ & $\begin{array}{c}0.0836^{* *} \\
(0.028)\end{array}$ & $\begin{array}{c}0.0792 * * \\
(0.028)\end{array}$ & $\begin{array}{c}0.0813^{* *} \\
(0.028)\end{array}$ & $\begin{array}{c}0.0767 * * \\
(0.028)\end{array}$ \\
\hline Parental Educ & & $\mathrm{Y}$ & & Y & & Y & & $\mathrm{Y}$ & & Y & & Y & & Y & & Y \\
\hline $\mathrm{R}^{2}$ & 0.181 & 0.183 & 0.183 & 0.184 & 0.183 & 0.184 & 0.184 & 0.185 & & & & & & & & \\
\hline Obs. & 20058 & - & - & - & - & - & - & - & - & - & - & - & - & - & - & - \\
\hline \multicolumn{17}{|l|}{ Panel B: Male } \\
\hline Educ & $\begin{array}{c}0.0784^{* * * *} \\
(0.011)\end{array}$ & $\begin{array}{c}0.0706^{* * *} \\
(0.012)\end{array}$ & $\begin{array}{c}0.0372 * \\
(0.015)\end{array}$ & $\begin{array}{c}0.0294 * \\
(0.015)\end{array}$ & $\begin{array}{c}0.0762 * * * \\
(0.012)\end{array}$ & $\begin{array}{c}0.0688^{* * * *} \\
(0.012)\end{array}$ & $\begin{array}{c}0.0365^{*} \\
(0.015)\end{array}$ & $\begin{array}{c}0.0290^{*} \\
(0.015)\end{array}$ & $\begin{array}{c}0.0734^{* * *} \\
(0.012)\end{array}$ & $\begin{array}{c}0.0671 \text { *** } \\
(0.012)\end{array}$ & $\begin{array}{c}0.0398^{* *} \\
(0.015)\end{array}$ & $\begin{array}{c}0.0330^{*} \\
(0.015)\end{array}$ & $\begin{array}{c}0.0713 * * * \\
(0.012)\end{array}$ & $\begin{array}{c}0.0645^{* * *} \\
(0.012)\end{array}$ & $\begin{array}{c}0.0361^{*} \\
(0.015)\end{array}$ & $\begin{array}{c}0.0291^{*} \\
(0.015)\end{array}$ \\
\hline Educ $\times$ College & & & $\begin{array}{l}0.0219 \\
(0.045)\end{array}$ & $\begin{array}{l}0.0221 \\
(0.045)\end{array}$ & & & $\begin{array}{l}0.0202 \\
(0.045)\end{array}$ & $\begin{array}{l}0.0206 \\
(0.045)\end{array}$ & & & $\begin{array}{l}0.0240 \\
(0.047)\end{array}$ & $\begin{array}{l}0.0240 \\
(0.046)\end{array}$ & & & $\begin{array}{l}0.0112 \\
(0.046)\end{array}$ & $\begin{array}{l}0.0119 \\
(0.045)\end{array}$ \\
\hline Income PGI & & & & & $\begin{array}{c}0.0271^{*} \\
(0.014)\end{array}$ & $\begin{array}{l}0.0242 \\
(0.014)\end{array}$ & $\begin{array}{l}0.0228 \\
(0.014)\end{array}$ & $\begin{array}{l}0.0199 \\
(0.014)\end{array}$ & $\begin{array}{c}0.2020^{*} \\
(0.090)\end{array}$ & $\begin{array}{c}0.1924 * \\
(0.090)\end{array}$ & $\begin{array}{c}0.1864 * \\
(0.090)\end{array}$ & $\begin{array}{l}0.1762 \\
(0.090)\end{array}$ & $\begin{array}{c}0.1057^{*} \\
(0.046)\end{array}$ & $\begin{array}{c}0.1008^{*} \\
(0.046)\end{array}$ & $\begin{array}{c}0.0970^{*} \\
(0.046)\end{array}$ & $\begin{array}{c}0.0918^{*} \\
(0.046)\end{array}$ \\
\hline Parental Educ & & Y & & Y & & Y & & Y & & Y & & Y & & $\mathrm{Y}$ & & Y \\
\hline $\mathrm{R}^{2}$ & 0.105 & 0.108 & 0.111 & 0.114 & 0.106 & 0.109 & 0.111 & 0.114 & & & & & & & & \\
\hline Obs. & 8310 & - & - & - & - & - & - & - & - & - & - & - & - & - & - & - \\
\hline \multicolumn{17}{|l|}{ Panel C: Female } \\
\hline Educ & $\begin{array}{c}0.1330 * * * \\
(0.009)\end{array}$ & $\begin{array}{c}0.1295^{* * * *} \\
(0.010)\end{array}$ & $\begin{array}{c}0.1273 * * * \\
(0.014)\end{array}$ & $\begin{array}{c}0.1230^{* * * *} \\
(0.015)\end{array}$ & $\begin{array}{c}0.1291 * * * \\
(0.010)\end{array}$ & $\begin{array}{c}0.1259^{* * *} \\
(0.010)\end{array}$ & $\begin{array}{c}0.1238^{* * * *} \\
(0.014)\end{array}$ & $\begin{array}{c}0.1198 * * * \\
(0.015)\end{array}$ & $\begin{array}{c}0.1263^{* * *} \\
(0.011)\end{array}$ & $\begin{array}{c}0.1229^{* * *} \\
(0.011)\end{array}$ & $\begin{array}{c}0.1209^{* * *} \\
(0.016)\end{array}$ & $\begin{array}{c}0.1166^{* * * *} \\
(0.016)\end{array}$ & $\begin{array}{c}0.1253^{* * *} \\
(0.011)\end{array}$ & $\begin{array}{c}0.1224 * * * \\
(0.011)\end{array}$ & $\begin{array}{c}0.1208^{* * *} \\
(0.015)\end{array}$ & $\begin{array}{c}0.1170^{* * *} \\
(0.016)\end{array}$ \\
\hline Educ $\times$ College & & & $\begin{array}{c}0.1013 * \\
(0.043)\end{array}$ & $\begin{array}{c}0.0995^{*} \\
(0.043)\end{array}$ & & & $\begin{array}{c}0.0996^{*} \\
(0.043)\end{array}$ & $\begin{array}{c}0.0981 * \\
(0.042)\end{array}$ & & & $\begin{array}{c}0.0991 * \\
(0.043)\end{array}$ & $\begin{array}{c}0.0973^{*} \\
(0.043)\end{array}$ & & & $\begin{array}{c}0.0978^{*} \\
(0.043)\end{array}$ & $\begin{array}{c}0.0964 * \\
(0.043)\end{array}$ \\
\hline Income PGI & & & & & $\begin{array}{c}0.0315^{* *} \\
(0.011)\end{array}$ & $\begin{array}{c}0.0303^{* *} \\
(0.011)\end{array}$ & $\begin{array}{c}0.0311^{* *} \\
(0.011)\end{array}$ & $\begin{array}{c}0.0299 * * \\
(0.011)\end{array}$ & $\begin{array}{l}0.1263 \\
(0.067)\end{array}$ & $\begin{array}{l}0.1179 \\
(0.066)\end{array}$ & $\begin{array}{l}0.1220 \\
(0.067)\end{array}$ & $\begin{array}{l}0.1138 \\
(0.066)\end{array}$ & $\begin{array}{l}0.0675 \\
(0.035)\end{array}$ & $\begin{array}{l}0.0636 \\
(0.035)\end{array}$ & $\begin{array}{l}0.0652 \\
(0.035)\end{array}$ & $\begin{array}{l}0.0614 \\
(0.035)\end{array}$ \\
\hline Parental Educ & & Y & & Y & & Y & & $\mathrm{Y}$ & & Y & & Y & & Y & & Y \\
\hline $\mathrm{R}^{2}$ & 0.151 & 0.152 & 0.152 & 0.153 & 0.152 & 0.153 & 0.153 & 0.154 & & & & & & & & \\
\hline Obs. & 11748 & - & - & - & - & - & - & - & - & - & - & - & - & - & - & - \\
\hline
\end{tabular}

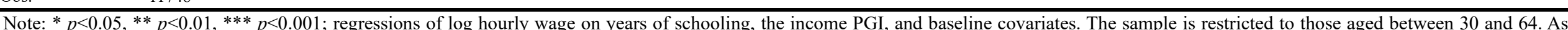




covariates while the other columns report the baseline model with the PGI added using different approaches. In the parentheses, we report standard errors clustered by individuals. 


\title{
Online appendix to:
}

\section{Genetic Fortune: Winning or Losing Education, Income, and Health}

\author{
By Hyeokmoon Kweon, Casper A.P. Burik, Richard Karlsson Linnér, Ronald De Vlaming, \\ Aysu Okbay, Daphne Martschenko, K. Paige Harden, Thomas A. DiPrete, \\ and Philipp D. Koellinger
}

For Online Publication

\section{A.I. Imputing occupational wages from standardized occupation codes}

The UK Biobank (UKB) does not contain information about individual income. However, it provides rich information directly relevant to labor earnings such as 4-digit standardized occupation codes (SOCs) and working hours. We take advantage of this information to impute occupational wages in the UKB data. We use three datasets to develop our imputation algorithm: the Labour Force Survey (LFS) for the UK, the Annual Survey of Hours and Earnings (ASHE), and the British Household Panel Survey (BHPS), which was collected independently from the LFS. In short, we fit a regression model on wages in the LFS using mean and median wages for standardized job codes obtained from the ASHE. We then assess the accuracy of our imputation algorithm in the BHPS. As a final step, we apply the imputation algorithm in the UKB to obtain our measure of occupational wages.

\section{A.I.1. The Labour Force Survey (LFS)}

The LFS data are collected by the Office for National Statistics (ONS) of the UK. With the intention to be representative of the whole UK population, the LFS has selected samples every quarter by randomly drawing residential addresses from the postcode database since 1973. The households of those drawn addresses are followed for five consecutive quarters, where information on earnings is collected in the first and last waves. Covering a long period of years, the LFS provides data with a large sample size along with various sets of individual information relevant to labor earnings. We pool samples of wage-earning individuals aged between 35 and 64 observed 
from 2002 to 2016, given that the UKB sample consists of those aged 40-64. We include those aged 35-39 to increase the sample size of the LFS because these individuals should not be too different from those aged over 40.

In addition to those with incomplete data, we excluded those who reported working hours less than one hour per week as well as those who reported working part-time (full-time) but working for more than or equal to (less than) 30 hours per week. We exclude these observations as they only introduce more noise in the estimation. As a result, we obtain a sample size of 464,192.

\section{A.I.2. The Annual Survey of Hours and Earnings (ASHE)}

The ASHE, also collected by the ONS, provides detailed labor statistics of the UK working population. It is based on a $1 \%$ sample randomly drawn annually from employees recorded in the labor tax database of the UK. It is therefore the best source available from which high-quality wage information of each occupation can be obtained. The occupation groups are defined by the Standard Occupation Classification (SOC) constructed by the ONS. The classifications define four levels of occupation groups indexed by 4-digit codes. In the case of the 2000 version, the 4-digit SOC defines 353 occupation groups.

\section{A.I.3. The British Household Panel Survey (BHPS)}

Conducted by the Institute for Social and Economic Research at the University of Essex, the BHPS is an annual survey that provides data on a nationally representative sample of more than 5,000 households and their adult members, who were followed from 1991 to 2009, with additional subsamples added in 1997 and 1999. Similar to the LFS, the household samples were drawn randomly from the postcode database. Despite its smaller sample size compared to that of the LFS, the BHPS has rich information on working individuals, which can suitably be used to test the accuracy of wage imputation based on LFS data. We pool working individuals aged 40-64 observed from 2002 to 2009 , removing one extreme outlier with a peculiarly high wage. As it is done for the LFS, we also exclude those who reported working for less than one hour per week. A sample size of 32,947 is obtained as a result. 


\section{A.I.4. Occupation composition}

While the LFS and the BHPS consist of representative samples of the UK population, the participants of the UKB data were not recruited in such a way that they can exactly represent the UK population. This fact implies that using the BHPS to assess the imputation accuracy may lead to exceedingly optimistic results of the imputation accuracy to the extent that the imputation error occurs due to the sample difference between the LFS and the UKB. Therefore, it is important to first identify any distinctive differences between the data sets. Since it is practically impossible to discuss every summary statistic for all three data sets, we examine only the occupation composition of each data set.

Table A11 presents the mean wages of nine major occupation groups (defined by the 1-digit SOCs) obtained from the ASHE and averaged over 2006-2010, the period during which the UKB participants were surveyed. It shows that the top three major occupation groups are: "1. Managers and senior officials", "2. Professional occupations" and "3. Associate professional and technical occupations". On the other hand, the bottom three groups are: "7. Sales and customer service occupations", "9. Elementary occupations", and "6. Personal service occupations"

Figure A4 depicts the composition of these major occupation groups for each sample. Clearly, compared to the LFS and the BHPS, a larger share of the individuals in the UKB had a job classified in the top three occupation groups. It is also evident that there are relatively fewer individuals in the UKB who had a job that belongs to the bottom three occupation groups. In contrast, there is only a subtle difference between the LFS and the BHPS in their job composition, which is not a surprise as they are meant to represent the UK population. Hence, it is important to keep in mind that the UKB sample includes many individuals who had relatively high-paying jobs and their actual wage levels are likely to be higher on average than those in the LFS and the BHPS.

\section{A.I.5. Imputation approach}

Our imputation approach is fully parametric and conducted in two steps. We first fit a regression of $\log$ wages in the LFS data. Based on the estimated coefficients, we then predict log-wages for samples in the UKB. We impute both weekly and hourly wages because the latter demands less information while the former can be imputed more precisely. This procedure requires that the two data sets contain the same predictor variables. Additionally, the available predictor variables must be sufficiently correlated with the wage distribution. Given these requirements, it is possible to use 
the following predictors: 4-digit standardized occupation code (SOC), working hours, full/parttime status, age, sex, square, cube, and interaction terms, as well as year-specific effects.

The pivotal information for predicting wages is the occupational information given by the 4digit SOCs. However, simply including dummy variables for the 4-digit SOCs in the prediction model (353 occupation groups in total for the 2000 SOC) in combination with other categorical characteristics (e.g., age, sex) can lead to cells with zero or too few observations, which in turn will result in failed or inaccurate imputations for some of the sample. Therefore, instead of dummy variables, we include mean and median wages for each individual's occupation group by sex and full/part-time status obtained from the ASHE.

This is the key feature of our imputation procedure that ensures both the efficiency and the accuracy of the imputation. In particular, using wage data from the ASHE is useful in that it allows us to include interaction terms between occupational profiles and other variables without substantially increasing the number of parameters, which can efficiently capture any heterogeneity across occupational groups. Another important reason is a change in the SOC system made in 2010, which updated the SOC from its 2000 version to the 2010 version. As the job codes in the two SOC systems cannot straightforwardly be converted into the other system, in essence, it is impossible to pool the LFS data for a long period that covers both before and after 2010 if one wishes to make use of detailed occupational information. Complementing the LFS with the ASHE data offers a convenient solution to this complication.

Specifically, the prediction model is specified as:

$$
\log \left(Y_{i}\right)=\alpha+X_{i} \beta+Z_{i} \gamma+\text { interaction }_{i} \delta+\epsilon_{i}
$$

where $\log \left(Y_{i}\right)$ are log weekly gross wages from employment, standardized to the 2015 currency value by the Consumer Price Index. $X_{i}$ is a vector of predictors including working hours per week (and its square), and dummies for full/part-time status (full if working hours $\geq 30$ ), age, sex, year of observation, and 2-digit SOCs. ${ }^{1} Z_{i}$ is a vector that contains log mean and log median weekly wages as well as their interaction term for the 4-digit-level occupation group (by sex and full/part-

\footnotetext{
${ }^{1}$ While the variation over 4-digit level occupation groups is supposed to be captured by mean and median wages from the ASHE, including the dummies for occupational groups appears to still improve the model fit by correcting some group-level difference between the LHS and the ASHE data. The 2-digit level is chosen to have sufficient observations in each group. Additionally, the difference between the 2000 and 2010 SOC systems is not too problematic for the 2-digit level classifications.
} 
time status) to which individual $i$ belongs. "interaction $i$ " includes interaction terms between sex, full/part-time status, the remaining variables in $X_{i}$, and the $Z_{i}$ term.

The prediction model for hourly wages is identical except that the hours worked variable is used only to construct hourly wages from the weekly wages and the dummy for full/part-time status as well as hours worked are not included as predictors. Furthermore, occupational hourly wages are collected from the ASHE only by sex.

The imputation is then conducted as follows: $(i)$ estimate the prediction model in the LFS data using mean and median wages of each SOC obtained from the ASHE; (ii) predict log wages in the UKB sample based on the estimated coefficients from (i). To evaluate the imputation accuracy, we utilize the BHPS data by comparing the predicted log-wages from step $(i)$ for samples in the BHPS with their actual log-wages. By regressing the imputed log-wages on the actual log wages of the BHPS, we obtain an $R^{2}$ of approximately 0.75 for weekly wages and 0.50 for hourly wages. Figure A5 presents a scatter plot of the actual log wages against the imputed log wages, which demonstrates that the wages are imputed with reasonable accuracy. Note that the distribution of reported log wages may contain transitory variance; therefore, the reported imputation accuracy can be regarded as a conservative result.

\section{A.I.6. Imputing wages of the UKB}

We impute both weekly and hourly wages for individuals in the UKB. For the former, only the current job information is used since the information on hours worked is available only for the current job. Additionally, only individuals younger than 65 are considered. For the latter, we use primarily information on the current job, while we instead use the information on the most recent job held before the age of 65 if it is not reported or if an individual is older than 64. Therefore, whereas we impute weekly wages with more reliable information, we can obtain a larger sample size for hourly wages. As a result, we impute weekly wages for 236,743 individuals and hourly wages for 282,963 individuals.

Since the two imputed wages are both available for 236,743 individuals, the first check is to compare the two imputed wages as follows: we subtract the log of hours worked from the imputed log weekly wages, which effectively produces the log hourly wage. $R^{2}$ between this computed term and the imputed hourly wage is 0.92 , which implies that both of the wages are imputed with reasonable accuracy. 
We ran GWAS on both weekly and hourly wages imputed for the UKB. For the former, hours worked and their polynomial terms are also controlled for. We then confirmed that the genetic correlation between the two wage measures is not statistically distinguishable from one $(0.993$ with $S E=0.004)$, which means that the distributions of the GWAS estimates are almost the same. Because imputing hourly wages requires less information and allows us to have a larger sample size, we utilize only log hourly wages in this paper.

\section{A.II. Estimating heritability from SNP data}

\section{A.II.1. Heritability framework}

Consider the following general framework for the outcome of interest, $y$, for some individual, $i$ :

$$
y_{i}=g_{i}+e_{i}
$$

where $y_{i}$ is decomposed into a contribution from genes in the form of SNPs, denoted by $g_{i}$, and the environment, denoted by $e_{i}$. Genetic effects are modeled as $g_{i}=\mathbf{x}_{i}^{\prime} \boldsymbol{\beta}$ where $\mathbf{x}_{i}$ is the vector that comprises data on $J$ standardized SNPs for the given individual. Here, vector $\boldsymbol{\beta}$ represents the vector of causal SNP effects, which includes SNP effects that work indirectly e.g., via behavioral or environmental pathways. Finally, $e$ is a residual that can be interpreted as the nongenetic component of environmental influences.

This linear model is a simplified version of how genetic factors can contribute to an outcome by making two important assumptions. First, it abstracts from alleles that have dominant or recessive effects. $^{2}$ Second, it does not include possible gene-gene or gene-environment interactions. However, the weighted average effects of the alleles can be estimated with this linear model, which is a useful starting point.

The challenge is that the true vector $\boldsymbol{\beta}$ is unknown, and any estimate of $\boldsymbol{\beta}$ is noisy and potentially biased in finite population samples, especially if the genetic architecture of $y$ is complex and driven by many SNPs that each have very small effect sizes. However, one can still obtain an estimate of $h_{S N P}^{2}$ by considering whether two randomly drawn individuals who are genetically slightly more

\footnotetext{
${ }^{2}$ This assumption is often justified by pointing to existing data and theory that primarily suggest additive genetic effects for most genetically complex traits (W. G. Hill, Goddard, and Visscher 2008).
} 
similar to each other than expected by chance, also tend to resemble each other slightly more in terms of the given outcome.

More specifically, if we are able to compute genetic similarities between people, we can model the covariance between individuals in terms of outcomes as a function of genetic similarity. This approach enables us to decompose the total variance in $y$ into the variance from the genetic and environment components, denoted by $\sigma_{g}^{2}$ and $\sigma_{e}^{2}$ respectively, assuming that $g$ and $e$ are orthogonal (Yang et al. 2010; Visscher 2010).

Such a model can be extended to control for confounding factors, such as population structure, and can thus be formulated as a so-called linear mixed model (LMM), where SNPs are assumed to have linear random effects and the covariates are assumed to have linear, fixed effects.

Importantly, the term "fixed effects" has different meanings in different fields. In complex trait genetics (i.e., the field that considers outcomes to which many genes contribute) fixed effects are simply conceptualized as nonstochastic effects - they are parameters that have a fixed value in a population. However, in panel econometrics, fixed effects often refer to firm-, country- or individual-specific means that do not change over time. In the context of family data, fixed effects can be considered as family-specific means that do not change from one family member to the next. Here, we use the term fixed effect interchangeably. That is to say, when we talk about fixed effects in an LMM, we simply mean non-stochastic effects, and when we talk about fixed effects in a family study, we mean family-specific effects.

Under the formal derivations involved in such a SNP LMM, one typically assumes that the elements of $\boldsymbol{\beta}$ (i.e., the vector of effects of the standardized SNPs) are independent and identically distributed draws from a normal distribution with mean zero, and a variance equal to $\sigma_{\beta}^{2}$. This assumption should be thought of as a mild prior on the SNP effects which implies that a trait is influenced by many, if not all, SNPs, where, in turn, the contribution of each SNP separately is very small. Under this model, the true $h_{S N P}^{2}$ is given by the total genetic variance, $\sigma_{g}^{2}=J \sigma_{\beta}^{2}$, over the total phenotypic variance, after correcting for the covariates that are assumed to have fixed effects. That is, $h_{S N P}^{2}=\sigma_{g}^{2}\left(\sigma_{g}^{2}+\sigma_{e}^{2}\right)^{-1}$. 


\section{A.II.2. Genomic-relatedness-based restricted maximum likelihood estimation}

Under such an LMM, $h_{S N P}^{2}$ is typically estimated using restricted maximum likelihood estimation. This approach is referred to as genomic-relatedness-based restricted maximum likelihood (GREML) estimation (Daniel J. Benjamin et al. 2012).

A crucial and strong assumption of this framework is that the true linear combination of genetic effects towards the outcome, $g$, is orthogonal to $e$. As long as this assumption is satisfied, GREML tends to yield robust estimates of $h_{S N P}^{2}$ under many different types of genetic architectures, even if the effect sizes of SNPs are not strictly drawn from a normal distribution. This result holds true as long as the set of SNPs that are analyzed represent the true genetic architecture of the trait in terms of $M A F$ and $L D$ (Lee and Chow 2014; Evans et al. 2018).

GREML estimates of $h_{S N P}^{2}$ from population samples can be influenced by indirect genetic influences of biological relatives (e.g., parents) that are not included in the sample, but that influence the observed outcomes through their behavior and the environment they create or provide (Young et al. 2018). Strictly speaking, such indirect genetic influences are not part of the standard definition of heritability. Nevertheless, the $h_{S N P}^{2}$ GREML estimate that can be obtained from population samples reflects true genetic effects that influence the outcomes of the observed individuals either via biological or environmental channels or a combination of both.

We estimate the heritability of occupation wages in the UKB sample using GREML. We first estimate $h_{S N P}^{2}$ with a single genetic variance parameter that uses all available unrelated individuals with data on occupational wages (196,187 in total, including 93,666 males and 102,521 females). For this estimation, we used SNPs that were included in the HapMap3 reference panel (Altshuler, Gibbs, and Peltonen 2010) because this set of SNPs tends to be measured with high accuracy and provides good coverage of common genetic variation among humans of European ancestries. For SNPs that contain mostly redundant information (LD $R^{2}>0.9$ ), we keep only the SNP with the highest $\mathrm{MAF}^{3}{ }^{3}$ Furthermore, we exclude rare SNPs with $\mathrm{MAF}<1 \%$ because they cannot be measured with high accuracy, and each of them contributes only a marginal share of the overall genetic variation in the sample (Auton et al. 2015). As covariates, we use dummies for age and the year of observation; dummies that account for differences in the income imputation procedure and

\footnotetext{
${ }^{3}$ This procedure is called LD-pruning, which was performed using Plink software (Purcell et al. 2007). In principle, LD-pruning is not necessary for GREML to work properly, although it might increase the estimate slightly. See (Yang et al. 2017) for an additional discussion.
} 
genotyping and assessment processes; and the first 40 PCs from the genetic data (Marchini et al. 2015). We also include a male dummy and interact it with the whole set of covariates in the pooled analysis. To reduce the computational burden due to the large sample size, we take advantage of an efficient algorithm developed by (Loh et al. 2015).

An additional feature of GREML is that it allows us to decompose the total SNP-based heritability for different types of SNPs. Specifically, if the SNPs are partitioned into independent groups $k=1, \ldots, K$, the total genetic variance $\sigma_{g}^{2}$ equals $\sum_{k=1}^{K} \sigma_{g k}^{2}$, where $\sigma_{g k}^{2}=J_{k} \sigma_{\beta k}^{2}$ denotes the variance accounted for by the $J_{k}$ SNPs in group $k$. Thus, by parsing the total set of SNPs into categories of interest (e.g., by chromosome, MAF, or LD), one can learn about the molecular genetic architecture of $y$ without needing to estimate the effects of each SNP directly.

Partitioning SNPs in this manner implicitly allows SNP effect sizes to be drawn from different distributions for the various categories considered (e.g., rare SNPs may on average have considerably larger effects than common SNPs), thereby reducing the scope for bias in GREML estimates (Evans et al. 2018). Furthermore, as we indicated, one can easily correct for covariates in GREML, enabling us to control for important sources of bias such as population structure.

The multiple variance parameter models require the computation of many GRMs. Since this is the computationally most intensive step of the procedure, we further reduce computational costs by restricting the analyses to a subset of 24,000 randomly selected unrelated individuals. We explore two cases: First, we allow the variance to differ across different groups of SNPs with different genetic features. More specifically, we define four groups of SNPs depending on $i$ ) whether they are common (MAF $\geq 0.05$ ) or rare $(0.01<\mathrm{MAF}<0.05)^{4}$ and ii) whether they have a relatively high or low LD score, using the median LD score as the cutoff. ${ }^{5}$ Since we are allowing for different variances of different genetic features, we use all available SNPs except for lowfrequency ones $(\mathrm{MAF}<0.01)$. Second, we let the variance differ across autosomes, which therefore gives 22 groups of SNPs. For computational reasons, we limited analysis to SNPs included in the HapMap3 reference panel.

\footnotetext{
${ }^{4}$ Common SNPs mean that their minor alleles are frequently observed, which leads to larger variation at those SNP locations. If SNPs are rare, we do not find much variation at those locations across individuals of the given population.

${ }^{5}$ The degree of LD between two SNPs can be measured by the Pearson correlation coefficient while the degree of LD for a given SNP can be measured by the LD score, which is the sum of the Pearson correlation coefficients with other SNPs.
} 


\section{A.II.3. Result}

Table A5 reports the results. The $h_{S N P}^{2}$ estimates are $12.6 \%(S E=0.5 \%)$ for females and $10.3 \%$ $(S E=0.5 \%)$ for males, suggesting a higher heritability of income for females. Thus, the heritability of occupational wages is somewhat higher for females than for males in our sample. When both male and female samples are analyzed together, the pooled $h^{2}{ }_{\mathrm{SNP}}$ is estimated to be $10.3 \%(S E=$ $0.3 \%$ ). Note that the estimate for the pooled sample is not necessarily the weighted average between the two estimates for males and females because additional covariates are included to control for male-female heterogeneity.

Figure A6 reports the heritability estimates of occupational wages from four different groups of SNPs, clustering them by their minor allele frequencies and LD. These estimates can shed light on the molecular genetic architecture of income by suggesting whether the observed heritability is primarily due to common genetic variants, which are most likely to have small effects, or due to relatively rare variants that may have stronger effects (Gibson 2012). Furthermore, this "binning" of SNPs into groups avoids potential bias in the GREML estimates which could stem from violations of the assumption that the effect sizes of all SNPs in $R^{2}$ are drawn from the same distribution, irrespective of their LD and MAF (Evans et al. 2018). Our results imply that relatively common SNPs ( $M A F \geq 5 \%$ ) with above-median LD scores contribute most to the total heritability of occupational wages $\left(h^{2}{ }_{\mathrm{SNP}}=4.6 \%, S E=0.9 \%\right)$, followed by relatively rare SNPs $(1 \%<M A F$ $<5 \%)$ with below-median LD scores $\left(h^{2} \mathrm{SNP}=3.0 \%, S E=1.9 \%\right)$. In contrast, rare SNPs with abovemedian LD scores seem to play at most a minor role. The sum of these four point estimates in Figure A6 is $10.2 \%(S E=2.2 \%)$, which is very close to the pooled $h^{2}$ SNP estimate of $10.3 \%(S E$ $=0.3 \%$ ). Notably, there are many rare genetic variants with low LD that are currently not included in genotyping arrays and that are difficult or impossible to impute (McCarthy et al. 2016; Auton et al. 2015). The effects of such rare, low-LD SNPs are unobserved here, which could contribute to the gap between our $h^{2}$ SNP estimates and heritability estimates for income from twin studies (Witte, Visscher, and Wray 2014).

Figure $A 7$ plots the heritability estimates of each chromosome against the number of effective loci per chromosome. ${ }^{6}$ For a genetically highly complex trait that has a very large number of causal SNPs across the genome, one would expect that each chromosome's contribution to the total

\footnotetext{
${ }^{6}$ Effective loci refer to the set of SNPs that can be considered statistically independent of each other. We obtained the number of effective loci for each chromosome from (Lee, Wedow, et al. 2018).
} 
heritability is approximately proportional to the number of independent loci on the chromosome (i.e., the amount of information contained in the chromosome). Indeed, the results in Figure A7 show that chromosomes with more effective loci contribute more to the heritability of income than chromosomes with fewer effective loci. A naïve regression of the subheritability estimates of each chromosome on the number of effective loci yields a standardized coefficient of 0.72 (95\% CI: 0.41-1.02) and $R^{2}=0.5$, suggesting a robust positive relationship between the number of effective loci and contributions to the heritability of occupational wage, which leaves room for some variation of the importance of each chromosome. Overall, these results are consistent with the idea that occupational wage is a genetically highly complex trait that is influenced by a large number of SNPs with small effects. Finally, the sum of $h^{2}$ SNP estimates across chromosomes is $9.5 \%$ ( $S E$ $=1.5 \%)$, which is slightly lower than the pooled $h^{2}$ SNP estimate reported in Table A5 $(10.3 \%, S E$ $=0.3 \%$ ), but the $95 \%$ confidence intervals of both point estimates overlap, assuming asymptotic normality.

Our SNP-based heritability estimate of $\approx 10 \%$ for occupational wages is considerably lower than most previously reported twin-study heritability estimates for income (Taubman 1976b, [a] 1976; Hyytinen et al. 2019). While some of this divergence may be driven by imprecise point estimates, potential upward bias in twin studies, or differences in the samples and measures of income used, our results are consistent with many studies on other traits that find lower heritability estimates with SNP-based methods compared to classic twin studies (Witte, Visscher, and Wray 2014). Most likely, a part of the difference can be attributed to a downward bias in $h^{2}$ SNP estimates due to unobserved genetic markers such as very rare and structural genetic variants that are in low LD with the observed SNPs on current genotyping arrays. Indeed, studies on BMI and height showed that much of this so-called "missing heritability" can be recovered when rare and structural genetic variants are observed and taken into account (Auton et al. 2015; Wainschtein et al. 2019).

However, since $\mathbf{X}$ is the result of both the genetic and the social lottery, $h^{2}$ SNP estimates based on $\mathbf{X}$ in population samples such as the one we used here may also capture indirect genetics effects from unobserved family members (e.g., parents and siblings) that influence the outcomes of the study participants via their behavior and the environment they create (Kong et al. 2018; Young et al. 2018). These so-called genetic nurturing effects violate the assumption of our estimation method that genetic effects and exogenously given environmental effects are orthogonal to each other. Genetic nurture effects are likely to be particularly relevant for socioeconomic outcomes 
such as income, and they are an interesting source of inequality by themselves (Koellinger and Harden 2018). Indirect genetic effects via relatives induce an upward bias in the $h^{2}$ SNP estimate.

Our within-family analyses using PGI shed some light on whether such indirect effects exist and how important they are.

\section{A.III. Genome-wide association study (GWAS) on occupational wages}

We follow a preregistered analysis plan (https://osf.io/rg8sh/) and conduct GWAS on occupational wages in the UKB. A GWAS systematically scans all measured genetic variations among people for associations with outcomes of interest. Thanks to rapid decreases in genotyping costs and a correspondingly rapid increase in the availability of genetic data, GWAS on thousands of traits have been conducted, enabling a remarkable range of discoveries in population and complex-trait genetics as well as epidemiology and the social sciences (Visscher et al. 2017; Harden and Koellinger 2020).

Consider Equation A2 again. In medical research, interest in $\boldsymbol{\beta}$ stems primarily from the hope of gaining insights into the biological causes of diseases that could potentially be targeted by drugs or other treatments (Visscher et al. 2017; King, Davis, and Degner 2019). In the context of socioeconomic differences, however, estimates of $\boldsymbol{\beta}$ are not only a useful starting point for biological investigations, but also may help to better understand the influence of individual behavior and the environment (Harden and Koellinger 2020; D. J. Benjamin et al. 2012).

The statistical challenge is that the true vector $\boldsymbol{\beta}$ is unknown and any estimate of $\boldsymbol{\beta}$ (denoted $\mathbf{b}$ ) is noisy and potentially biased in finite population samples, especially if the genetic architecture of $y$ is complex and driven by many SNPs that each have very small effect sizes (Chabris et al. 2015). Moreover, the number of SNPs $J$ is typically orders of magnitude greater than the number of individuals in the sample $(N)$. Therefore, Equation A2 cannot be estimated by fitting all SNPs simultaneously in a multiple regression. Instead, the outcome is regressed on each SNP separately, resulting in $J$ regressions in total. As a consequence, the LD-structure between SNPs (i.e., correlation between regressors) is ignored, and GWAS estimates should be considered as pointers to LD-linked regions in the genome (so-called loci) that are associated with the outcome rather than causal estimates.

More importantly, uncontrolled correlations between allele frequencies, environments, and ancestry backgrounds violate the assumption that $\mathbf{x}_{i}$ and $e$ are orthogonal, making it challenging to 
interpret GWAS estimates of $\mathbf{b}$ from population samples (Hamer and Sirota 2000; Young et al. 2019). The standard approach to tackle this challenge is (1) to restrict the sample to individuals of similar ancestries (due to data availability, this typically means European ancestries ${ }^{7}$ ) and (2) to control for leading principal components (PC) from genetic data in our GWAS. These lead PCs tend to capture differences in genotypes across geographic regions and ancestries, provided they are constructed using a large set of uncorrelated SNPs (Price et al. 2006; Abdellaoui et al. 2013). We follow this state-of-art approach here and conduct a GWAS on the log of occupational wages, including 40 genetic PCs as control variables to adjust for population stratification bias and modeling the error structure with random SNP effects to account for relatedness.

It is crucial to understand that $\mathbf{b}$ is not a clean estimate of biological effects for two reasons. First, $\mathbf{b}$ includes indirect effects of $\mathbf{x}_{i}$ on $y_{i}$ that work via behavioral or environmental pathways (e.g., self-selection of $i$ into specific environments and feedback loops between the behavior of $i$ and the responses from those environments). Thus, b partly reflects social facts that depend on environmental conditions and may vary across different environments and samples. In our context, technological progress, labor market conditions, and social factors such as discrimination or racism may influence the $\mathbf{b}$ for income. Thus, the effects of $\mathbf{x}_{i}$ on $y_{i}$ that are captured by $\mathbf{b}$ are not universally true or simply "given by nature". Rather, b reflects social and economic realities that may be outside of the control of any given individual $i$ but are subject to change over time and could be malleable by collective human action, policy reforms etc. Which specific environmental factors influence $\mathbf{b}$ is an empirical question of considerable interest for the social sciences and policy.

Second, $\mathbf{x}_{i}$ is the outcome of both the genetic and the social lottery in a population sample, which implies that $\mathbf{b}$ captures both genetic and family-specific effects. To isolate the effects of the genetic and the social lottery, it would be necessary to estimate $\mathbf{b}$ from random differences in $\mathbf{x}_{i}$ between biological relatives (e.g., siblings or parents and their children). However, doing so would sharply reduce the GWAS sample size $N$ and the variance in $\mathbf{x}_{i}$ and $y_{i}$, which would lead to a substantial

\footnotetext{
${ }^{7}$ Between 2007 and 2017, 86\% of GWAS discovery samples were of European ancestries (Mills and Rahal 2019) partly because the interest and investments in genetic research vary dramatically across countries and more than $50 \%$ of all GWAS participants until 2017 were recruited in the UK and Iceland alone - two countries that are populated by a majority of people of European ancestries. However, factors such as distrust of the medical/scientific community, poor access to primary medical care, the failure of researchers to actively recruit non-Europeans, the alienation of minority health professionals, the lack of knowledge about clinical trials, as well as language and cultural barriers were also identified as important impediments to more diverse genotyped research samples (Shavers-Hornaday et al. 1997).
} 
loss of statistical power to estimate $\mathbf{b}$. In the absence of extremely large genotyped family samples, the best strategy to estimate $\mathbf{b}$ is arguably to maximize the statistical power by maximizing $N$ with population samples, followed by within-family analyses that make use of polygenic indices that have been constructed from estimates of $\mathbf{b}$ in independent samples (Chabris et al. 2015; Lee, Wedow, et al. 2018; Kong et al. 2018). We follow this strategy in the current study.

Specifically, we conduct GWAS on occupational wages in the UKB population sample using a linear mixed model (LMM). In our LMM, we assign a fixed effect to the SNP under consideration, while treating the effects of all other SNPs as random (Yang et al. 2014). In addition, we control for the 40 leading PCs from the genetic data in our LMM.

For each SNP $j=1, \ldots, J$, we consider a leave-one-chromosome-out (LOCO) LMM for our sample of $N$ individuals. That is, we examine

$$
\mathrm{y}=\mathrm{x}_{\mathrm{j}} \beta_{\mathrm{j}}+\mathrm{Z} \boldsymbol{\gamma}+\mathrm{X}_{(\mathrm{j})} \boldsymbol{\beta}_{(\mathrm{j})}+\mathrm{e}
$$

where y denotes the outcome vector and $\mathrm{x}_{\mathrm{j}}$ the $N$-by-1 vector of data on SNP $j$. The main parameter of interest is $\beta_{j}$, the effect of SNP $j$. In this model, Z denotes the N-by-K matrix of covariates, with effects $\boldsymbol{\gamma}$, and $\mathrm{X}_{(\mathrm{j})}$ denotes the matrix of SNP data, excluding the SNP at hand as well as all other SNPs on the same chromosome, with associated random effects $\boldsymbol{\beta}_{(\mathrm{j})}$.

Effectively, under this LOCO LMM, we have an error structure that captures contributions from the environment as well as SNPs located on other chromosomes. This error structure is fully characterized by the two parameters $\sigma_{g}^{2}$ and $\sigma_{\varepsilon}^{2}$. We estimate this LOCO LMM in two steps. First, the parameters $\sigma_{g}^{2}$ and $\sigma_{\varepsilon}^{2}$ are estimated using GREML (i.e., the same method that is also used for $h_{S N P}^{2}$ estimation). Second, the effects, $\beta_{\mathrm{j}}$ and $\boldsymbol{\gamma}$, are then estimated with generalized least squares. To reduce the computational burden, we use an efficient approximation algorithm that converges after a small number of iterations (Loh et al. 2015).

To account for the possibility that occupational wages are influenced by different sex-specific factors, we estimate GWAS according to Equation A3 separately for males $(N=133,689)$ and females $(N=149,274)$ using the same set of covariates as in the heritability estimation described above. Next, we combined the sex-specific results using a special case of the MTAG (multi-trait 
analysis of GWAS) method. ${ }^{8}$ MTAG implements a generalized version of inverse-varianceweighted meta-analysis that takes into account the relatedness between the male and female samples as well as potential bias due to an uncontrolled population structure (Turley et al. 2018). ${ }^{9}$

Several quality control filters were applied to exclude SNPs that are problematic, implemented according to the commonly applied procedure developed by (Winkler et al. (2014)). The main steps include removing SNPs that have missing or incorrect numerical values for some variables (a $p$-value outside of $[0,1]$, for instance); have a MAF below $0.1 \%$; have imputation accuracy below 0.7 ; have the effect-coded allele or the other allele with values different from "A," "C," "G," or "T"; are duplicate SNPs; and have an allele frequency different from the allele frequency in the HRC reference panel by more than 0.2 .

A systematic scan of all observed SNPs in a GWAS imposes a high multiple-testing burden, which led to the adoption of a stringent $p$-value threshold of $5 \times 10^{-8}$ for genome-wide significance, reflecting a Bonferroni correction for one million independent tests (Shah et al. (2008)). Although the actual number of SNPs in a GWAS is often higher than one million, many SNPs are correlated, and the $p$-value threshold of $5 \times 10^{-8}$ is the accepted benchmark for GWAS in European ancestry samples that rely primarily on common SNPs. ${ }^{10}$

We follow this standard approach here. Of the 9,773,980 autosomal SNPs included in our GWAS on occupational wages in the UKB $(N=282,963)$, we identified 3,920 genome-wide

\footnotetext{
${ }^{8}$ The similarity of the genetic architecture of income for males and females can be quantified by the genetic correlation coefficient. A robust estimate of this quantity can be obtained with bivariate LD score regression (CanelaXandri, Rawlik, and Tenesa 2018; Lee, McGue, et al. 2018). Applying this method to our GWAS results on income in the UKB, we estimate a genetic correlation of $0.921(S E=0.034)$ between males and females, which is very high but statistically indistinguishable from a perfect genetic correlation of 1 (Koellinger et al. 2018).

${ }^{9}$ A measure of bias due to population stratification can be obtained from the intercept, a so-called LD score regression, which regresses the LD scores of all SNPs on their observed Chi-squared test statistic in a GWAS (B. K. Bulik-Sullivan et al. 2015).

${ }^{10}$ More stringent thresholds may be necessary for non-European ancestries that observe a higher degree of genetic variation and datasets that include very rare or structural genetic variants that are not in strong LD with common SNPs (Auton et al. 2015).
} 
significant SNPs. These 3,920 SNPs cluster across 45 approximately independent loci. ${ }^{11}$ We found one novel locus that has never been reported for any other traits. ${ }^{12}$

The GWAS results are visually presented in a so-called Manhattan plot in Figure A8, where the $p$-values of all SNPs are plotted on a $-\log _{10}$ scale against their chromosomal position. The SNPs with the lowest $p$-value per locus are referred to as lead SNPs and are reported in Table A6. The effect sizes of the lead SNPs in absolute terms range from 0.018 to 0.005 with a mean of 0.007 , which corresponds to a $0.7 \%$ change in occupational wage per allele. ${ }^{13}$ In terms of $R^{2}$, the variance explained by each lead SNP ranges from $0.011 \%$ to $0.037 \%$ with a mean of $0.014 \%$, implying that each of the lead SNPs captures only a very small proportion of the variation in occupational wages. Note that uncontrolled population structure (Price et al. 2006), indirect genetic effects (Kong et al. 2018), and the statistical winner's curse would tend to bias these effect size estimates away from zero (Palmer and Pe'er 2017), which implies that the true causal effects may be even smaller. The genomic loci that are represented by our lead SNPs can be positionally mapped to 184 proteincoding genes. An analysis of the set of genes that are potentially tagged by our GWAS results shows that they are most strongly expressed in brain tissues. ${ }^{14}$

We compared our results to GWAS results for other SES measures by using the 45 lead SNPs as well as the SNPs that are highly correlated with them $\left(R^{2}>0.6\right)$. Twenty-six of our loci were previously found to be significantly associated with household income (W. D. Hill et al. 2019) and 31 loci with educational attainment (Lee, Wedow, et al. 2018). The similarity of the distribution of SNP effects for two different traits $\left(\boldsymbol{\beta}_{1}, \boldsymbol{\beta}_{2}\right)$ is given by the so-called genetic correlation coefficient (B. Bulik-Sullivan et al. 2015; Okbay et al. 2016). The genetic correlation of occupational wages with educational attainment is $0.923(S E=0.01)$ and $0.919(S E=0.02)$ with

\footnotetext{
${ }^{11}$ We define a locus using a clumping algorithm that begins by selecting the SNP with the lowest p-value as the lead SNP in the first clump and includes in the first clump all SNPs that have $R^{2}$ greater than 0.1 with the lead SNP. Next, the SNP with the second-lowest $p$-value outside the first clump becomes the lead SNP of the second clump, and the second clump is created analogously but using only the SNPs outside of the first clump. This process continues until every genome-wide-significant SNP is either designated a lead SNP or is clumped to another lead SNP. The genomic proximity is not considered when forming clumps. The LD between the SNPs is calculated from a reference panel of the Haplotype Reference Consortium (HRC; (McCarthy et al. 2016))

${ }^{12}$ We looked up the previous GWAS results by using the GWAS Catalog data obtained on February 212020 (Buniello et al. 2019).

${ }^{13}$ These effect size estimates are not LD-adjusted.

${ }^{14}$ Functional mapping and annotation of our GWAS results was conducted using the bioinformatic tool FUMA (Watanabe et al. 2017).
} 
household income, which is statistically distinguishable from one. ${ }^{15}$ Thus, the genetic architecture of these different measures of SES is very similar, but not exactly identical.

Most of the genome-wide significant loci identified here were previously found to be associated with health outcomes such as BMI, HDL cholesterol levels, diabetes, bipolar disorder, alcohol consumption, Parkinson's disease, and many others (Table A6). Thus, genetic factors linked to income also tend to have relevance for health and vice versa. Several different mechanisms could lead to this relevance (Solovieff et al. 2013; Hemani, Bowden, and Davey Smith 2018). First, it is possible that some genes affect both income and health directly via the same biological mechanism. Second, health and income may act as mediating variables for each other. For example, income could have causal downstream consequences on health or health conditions, with early onset conditions (e.g., ADHD) possibly having causal effects on income. Finally, the identified genes may also be associated with unobserved outcomes that influence both health and income (e.g., neighborhood quality during childhood). GWAS results by themselves do not illuminate causal pathways, but the identified genetic loci can serve as useful starting points for follow-up studies that aim at elucidating mechanisms (Visscher et al. 2017; Harden and Koellinger 2020). Thus, although we do not know why our GWAS identifies these loci for income, it is clear that the genetic architectures of income and health are related and no clear boundaries can be drawn between socioeconomic and medical outcomes. This is not surprising, given the well-known relationships between SES and health (Wilkinson and Marmot 2003; Stringhini et al. 2017; Piotrowska et al. 2015; Chetty et al. 2016).

Although previous GWAS on household income or neighborhood differences (W. D. Hill et al. $2016,2019)$ uncovered many interesting findings, it is challenging to interpret genetic associations with such aggregate measures of prosperity. Our approach that maps individual differences in income to individual-specific genetic markers reduces this complexity to some extent and our results allow for the formation of new types of genetically informed study designs on income inequality (e.g., to estimate the returns to schooling while explicitly controlling for genetic confounds). Furthermore, although the most recent GWAS on educational attainment (Lee, Wedow, et al. 2018) of more than 1.1 million individuals was the largest GWAS on SES to date,

\footnotetext{
${ }^{15}$ The genetic correlation is the correlation between two sets of standardized GWAS effect sizes. We estimated this by using LD score regression (B. Bulik-Sullivan et al. 2015). Testing whether these estimates are different from one yields $p$-values of $5.03 \times 10^{-13}$ and $3.78 \times 10^{-8}$ respectively for educational attainment and household income, which indicates that the genetic correlation is not perfect.
} 
a genetic risk score constructed from our results improves upon the variance in individual income that can be captured by a risk score for educational attainment (see A.IV. Polygenic indices).

Since our GWAS results are derived from a sample of elderly inhabitants of the UK who all have European ancestries, there will be limits to the transferability of our results to other populations: The genetic associations with income we report here are conditional on the social and economic context of the White, educated, industrialized, rich, and democratic (Weird) sample we studied. Different contexts (e.g. discrimination against some groups in society) may imply different genetic architectures that would limit the external validity of our results (de Vlaming et al. 2017; Mostafavi et al. 2020). In addition, genetic associations also depend on the frequencies of genetic variants and their correlations with each other in the samples studied. This dependence generally limits the transferability of GWAS results and polygenic indices across groups that differ in ancestries (Martin et al. 2019; Rosenberg et al. 2019) and implies that our results cannot be used for comparison across groups. Our results cannot be used for predicting individual outcomes for the same reasons and for the limited statistical accuracy of our polygenic indice. (See FAQ sections "Can your polygenic score be used to predict how well someone will do in life?" and "Can your polygenic score be used for research studies in non-European-ancestry populations?”)

\section{A.IV. Polygenic indices}

The tiny effect sizes of each individual SNP in our GWAS on occupational wages $\left(R^{2}<0.04 \%\right)$ prohibit a statistically well-powered SNP-level replication of our results in the two available holdout samples. ${ }^{16}$ As an alternative, holistic form of replication, we constructed PGI from our GWAS estimates on occupational wages in the HRS and the WLS samples to test the associations with measures of self-reported income.

Our PGI uses LDpred-adjusted GWAS estimates, based on the observed LD structure in the prediction sample (Vilhjálmsson et al. 2015). Specifically, we construct PGI using all directly genotyped SNPs and those included in the HapMap3 reference panel (Altshuler, Gibbs, and Peltonen 2010), yielding 2,547,062 SNPs in the HRS, 1,519,416 SNPs in the WLS, and 1,685,746 SNPs in the UKB. This focus on common, high-quality SNPs improves the signal-to-noise ratio

\footnotetext{
16 The combined number of individuals in HRS and WLS that have genetic data, information on income, and standard control variables is $N=13,558$ (Table A2). This would yield only $\approx 50 \%$ statistical power to replicate a SNP with $R^{2}=0.04 \%$ at $\alpha=0.05$ and only $\approx 15 \%$ power for a SNP with $R^{2}=0.01 \%$.
} 
in the PGI relative to methods that use all available SNPs or only a small subset of them (e.g., only the SNPs that are genome-wide significant). ${ }^{17}$

The EA PGI are constructed from a publicly available version of the GWAS results reported in (Lee, Wedow, et al. 2018), which are based on $N \approx 760,000$, after regenerating GWAS results excluding each prediction sample. ${ }^{18}$

All PGIs are standardized to have zero mean and unit variance and tested in linear regressions on log hourly wages. The main quantity of interest here is the contribution of the PGI to variation in log hourly wages in a regression model with baseline controls. This contribution is measured by an increase in $R^{2}\left(\Delta R^{2}\right)$ in response to adding the PGI to a baseline model that controls e.g., for age, sex, the first 20 principal components of the genetic data. We also report partial $R^{2}$, the variance explained by the PGI when partialling out the control variables from both the log hourly wage and the PGI. For comparison, we also constructed a PGI for educational attainment (EA) in HRS and WLS using the results from a much larger GWAS sample $(N \approx 0.76$ million) that excluded the prediction samples (Lee, Wedow, et al. 2018). This PGI for EA has previously been shown to be correlated with labor market outcomes and measures of financial wealth in the HRS (Papageorge and Thom 2019; Barth, Papageorge, and Thom 2020). All PGIs are standardized to have zero mean and unit variance.

The polygenic prediction results for the HRS can be found in Panel A of Table A7. The pooled baseline model with all control variables but without the PGI captures $8.6 \%$ of the variance in $\log$ hourly wages. When the PGI for income is added to the model, $R^{2}$ increases by $0.91 \%$, giving a partial $R^{2}$ of $0.99 \%\left(p=6.2 \times 10^{-50}\right)$. This finding is in line with theoretical expectations that take the imprecision of the SNP effect-size estimates due to the finite GWAS sample size into account. ${ }^{19}$ The coefficient estimate for the income PGI implies that one-standard deviationincrease in the PGI is associated with an $8.0 \%$ increase in hourly wages (95\% CI: $6.2-9.7 \%)$.

\footnotetext{
${ }^{17}$ It is difficult to accurately measure and impute rare SNPs. Furthermore, the standard error of the estimated effect of rare SNPs is larger because $S E(b) \approx 2 \cdot M A F \cdot(1-M A F)$;see (Rietveld et al. 2013).

${ }^{18}$ Note that whenever constructing a PGI the GWAS summary statistics are regenerated to exclude a prediction sample, which prevents overfitting. Therefore, the actual sample size of EA GWAS used for PGI is slightly different for each prediction sample.

${ }^{19}$ Using the MetaGAP calculator (de Vlaming et al. 2017) with a GWAS sample size of $N=282,963$ (corresponding to the size of our UKB sample), 250,000 independent SNPs (a realistic number after GWAS quality control), and assuming $h^{2}{ }_{\text {SNP }}=0.1$ with 20,000 independent causal SNPs as well as a perfect genetic correlation between the GWAS and the prediction sample, the expected $R^{2}$ of a polygenic indice is $1 \%$.
} 
The EA PGI is also associated with income in the HRS $\left(\Delta R^{2} \approx 1.10 \%\right.$ in the pooled model; $p=$ $4.4 \times 10^{-60}$ ). If both PGI are included simultaneously, both remain predictive and statistically highly significant, jointly accounting for $1.29 \%$ of the variation in the hourly wage in the pooled HRS sample ( $p=2.8 \times 10^{-12} ; p=1.6 \times 10^{-22}$ ). Thus, the income PGI contributes information over and above the information in the EA PGI, although income and EA are genetically very similar (W. D. Hill et al. 2019; Koellinger et al. 2018) and the EA GWAS sample is substantially larger. This result likely occurred because the estimation error for the SNP effects is still relatively large, but not identical for both GWAS samples. Thus, having GWAS results for multiple indicators is a way to increase the combined predictive accuracy that can be obtained from genetic data. ${ }^{20}$ Since the EA and income PGI are correlated with each other $(\rho \approx 0.54)$, the increase in the explanatory power in the combined model is smaller than the sum of $\Delta R^{2}$ 's from the models in which each PGI is added separately. Finally, both polygenic indices capture similar shares in income variation for males and females in the HRS sample.

Panel B of Table A7 reports the results for self-reported wage rates observed in the WLS. While the results are consistent with the HRS in that we find statistically significant associations between the log hourly wage and the PGI in every case, the predictive power of the PGI is overall lower in the WLS. ${ }^{21}$ The pooled baseline model explains $19.2 \%$ of the variance and adding the income PGI increases the $R^{2}$ by $0.57 \%$. The coefficient estimate implies that an increase in the PGI of one standard deviation is associated with an increase of approximately $5.8 \%$ (95\% CI: 4.3-7.4) in the hourly wage rate. When using the EA PGI instead, the increase in $R^{2}$ is again slightly higher compared to the income PGI $\left(\Delta R^{2}=0.62 \%\right.$ and partial $\left.R^{2}=0.76 \%\right)$. Similar to what we observed in HRS, both PGI remain individually predictive and statistically highly significant $(p=$ $1.6 \times 10^{-4} ; p=1.9 \times 10^{-5}$ ) in a multiple regression that includes both scores, whose correlation is again $\rho \approx 0.54$.

\footnotetext{
${ }^{20}$ (Turley et al. 2018) developed a multivariate method to combine GWAS results from genetically strongly correlated traits that builds on a similar intuition. Indeed, adding several PGI as predictors in a multiple regression is a naïve way to mimic the increase in polygenic $R^{2}$ that their approach yields.

${ }^{21}$ The lower delta $R^{2}$ of the PGI in the WLS could be due to several factors, including a potentially lower $h^{2}$ of income in the WLS sample, a lower genetic correlation for income between the WLS and the UKB than between HRS and the UKB (de Vlaming et al. 2017), or because of the sibling pairs included in the WLS (the OLS estimates in WLS reflect within-family estimates to some degree, which tend to be smaller than between-family estimates, as shown in the following section).
} 
In contrast to the HRS sample, we observed considerable heterogeneity between males and females in the WLS in terms of $\Delta R^{2}$ of the PGI. While the females' wages observed in the WLS are as well predicted by the PGI as those in the HRS, the predictive accuracy of both PGI is lower for males. This result may be due to a lower SNP-based heritability of income for males in the WLS, but it may also be due to technical effects such as differences in the genetic architecture of income between males and females within and across the samples we studied.

In a similar vein, the predictive power of the PGI can also be underestimated due to the measurement error and transitory variance in the cross-sectional distribution of income. Table A8 reports the same set of analyses for the HRS but with 3-year moving averages of wages, which can alleviate such issues to some extent. In the pooled sample, $14.83 \%$ of the variation can be additionally explained by the income PGI. The income and educational attainment PGI together explain more than $2 \%$ of the variation. As such, the predictive power of the PGI is expected to be higher for better measures of income or longer-term income.

Although both our income PGI and the PGI for educational attainment capture a small share of the distribution of income in independent hold-out samples, they are not useful for individual-level predictions for statistical as well as conceptual reasons. The statistical limitations of individual predictions are illustrated in Figure A3. Even for PGI values that are relatively extreme outliers (e.g., those 2 standard deviations below or above the mean), an extremely wide range of incomes is observed. More generally, no single variable with an $R^{2} \approx 1 \%$ is useful for individual-level prediction. The $R^{2}$ of PGI will increase in the future as GWAS sample sizes increase (Daetwyler, Villanueva, and Woolliams 2008), with an upper bound given by $h^{2}$ SNP, which, according to our estimates, is in the range of approximately 10\% with current SNP data. However, even a variable with $R^{2} \approx 10 \%$ would not be sufficiently accurate to make reliable individual-level predictions (Harden and Koellinger 2020). As we emphasized before, PGI should not be considered as "objective" or "purely biological" measures. The GWAS results that are used to construct PGI may capture many things that are associated with income, but are not due to directly causal effects of genes (Haworth et al. 2019; Kong et al. 2018). To the extent that the income PGI capture directly causal genetic effects, these effects will reflect the social realities of the samples used in the study. For example, the environmental circumstances that matter in the context of income include the relative supply of and demand for certain types of skills (Acemoglu 2002); the presence or absence of discrimination (Bertrand and Mullainathan 2004; Reimers 1983); the extent to which problems 
of asymmetric information between employers, workers, and job candidates can or cannot be resolved (Autor 2001); existing regulations (Siebert 1997); the opportunity costs of labor market participation (Becker 1965), labor market discrimination (Kaas and Manger 2012) and many other features of economic reality that are neither universal nor necessarily optimal from a welfare perspective. Thus, aside from statistical considerations, the environmental context of the income distribution puts limits on the potential transferability of the income PGI across samples (Mostafavi et al. 2020). If PGI are used for individual prediction despite these limitations (e.g., in school entrance exams or job hires (Plomin 2019)), it can exacerbate existing stigmas, discrimination, and inefficiencies, and create or amplify economic and ethical dilemmas. Thus, while the PGI we constructed here can be a very useful tool for research, its practical implications are very limited at best and feature many complications.

\section{A.V. Additional information on data}

\section{Health and Retirement Study (HRS)}

In 2006, the HRS started collecting biomarkers and DNA samples in a subset of the paricipatns (Weir 2013). Here we used the second release of the HRS genetic data, ${ }^{22}$ which covers genotyping phases 1 to 3 . In phase 1, DNA samples were extracted from buccal swabs and in phase 2 and 3 this was done using saliva samples. The DNA samples were genotyped at the Center for Inherited Disease Research (CIDR). Phases 1 and 2 were genotyped together on the Illumina HumanOmni2.5-4v1 array and phase 3 was genotyped on the Illumina HumanOmni2.5-8v1 array.

The two arrays have 2,365,472 overlapping SNPs. Those SNPs were then subjected to CIDR technical filters and tests for duplicate sample discordance, Mendelian errors in trios, Hardy Weinberg equiibrium testing, and tests on sex differences. In total, 2,075,208 SNPs passed these QC filters (Weir 2013).

Autosomal SNPs were imputed using the worldwide reference panel from phase I of the 1000 Genomes project (v3, released March 2012) (Consortium and The 1000 Genomes Project Consortium 2012; HRS 2013). 1,945,761 Genotyped SNPs were used as a basis for imputation. Imputation was performed using IMPUTE2 software. The imputation output contains 22,378,417 autosomal SNPs. Before our analyses, we filtered out SNPs that had an imputation quality below

\footnotetext{
${ }^{22}$ See https://hrs.isr.umich.edu/data-products/genetic-data/products\#gdv2, for more information.
} 
0.7. SNPs were also removed if the SNP was missing in over 5\% of the sample or the $M A F$ was smaller than $1 \%$. Due to computational restraints in constructing the PGI with LDpred, we further reduced the number of SNPs to those that were directly genotyped or present in the HapMap3 imputation panel, providing us with a high-resolution coverage of common genetic variants. This leaves 2,547,062 SNPs included in the construction of the PGI.

Our analyses were restricted to unrelated participants of European descent. Specifically, HRS filtered out parent-offspring pairs, siblings and half-siblings. For participants present in version 1 of the HRS genetic data, we selected European descent using a list provided by HRS that is based on self-reported race and principal component analysis. For participants who were added to version 2 of the HRS genetic data, we excluded those who self-identified as having non-European ancestry as well as those who fall outside of the European ancestry cluster in our principal component analysis. The principal component analysis was performed by plotting the first four principal components of HRS version 1, together with HRS version 2 and the 1000 Genomes project reference panel. We also excluded individuals who did not satisfy HRS internal genotype quality control criteria.

\section{Wisconsin Longitudinal Study (WLS)}

The WLS (Herd, Carr, and Roan 2014) is a longitudinal study of individuals who graduated from Wisconsin high schools in 1957 as well as one randomly selected sibling. The original respondents and their selected siblings were surveyed six times over the years. The WLS collected extensive information on family backgrounds, schooling, and labor market experiences. In addition, genetic data was also collected for approximately 9,800 individuals including both the original respondents and their siblings. We use hourly wage rates surveyed in 1992-1994, which was the first wave during which information on wages was collected and the last wave before most respondents reached retirement age. The nominal wages are converted into real wages in the same way as in the HRS.

The WLS has two waves of genetic data, in our analyses we use the second wave of genetic data (Herd 2016). Over the course of 2007 and 2008 the WLS began to collect saliva samples by mail. Additional samples were added in 2010 during home interviews. A total of 9,027 panel members contributed saliva samples for genetic analysis. The DNA was extracted and genotyped at the CIDR using the Illumina HumanOmniExpress array (713,014 SNPs). 
Similar to the HRS genetic data the SNPs were subjected to CIDR technical filters and tests for duplicate sample discordance, Mendelian errors in trios, Hardy Weinberg Equiibrium testing, and tests on sex differences (Herd 2016).

In WLS, the autosomal SNPs were imputed using phase 3 from the 1000 Genomes project reference panel and imputation was performed using IMPUTE2 software (WLS 2016). This process resulted in 32,367,317 autosomal SNPs. In our PGI analyses, SNPs were selected in the same way as in the HRS data, resulting in 1,519,416 SNPs.

Our analyses were restricted to unrelated participants of European descent. Selection on European descent was done based on principal component analysis by plotting the first four principal components together with those of the 1000 Genomes project reference panel. Selfreported ethnicity was not considered here as it was surveyed only in a later wave, which led to too many missing outcomes.

\section{UK Biobank}

The UK Biobank genetic data contain genotypes for 488,377 participants (Bycroft et al. 2018). DNA was extracted from blood samples that were collected at a UK Biobank assessment center. A subset of 49,950 participants were genotyped using the UK BiLEVE Axiom Array by Affymetrix (807,411 markers). The remaining 438,427 participants were genotyped using the custom UK Biobank Axiom Array (825,927 markers). The two arrays have a 95\% overlap.

The markers were assessed on quality for batch, plate, array, or sex effects, tests on HardyWeinberg equilibrium and discordance across control replicates. Autosomal SNPs were imputed using a custom imputation panel based on the Haplotype Reference Consortium data and UK10K Panel using IMPUTE4 software. This resulted in an imputation output of 93,095,623 autosomal SNPs.

For the sibling sample used as a hold-out sample for PGI analyses, SNPs were selected in the same way as in the HRS data, resulting in 1,685,746 SNPs.

Our analyses were restricted to unrelated participants of European descent internally identified by the UKB. The UKB identified them based on principal component analysis with the 1000 Genomes project reference panel. The individuals were dropped if their reported ethnic background was not white. 


\section{References}

Abdellaoui, Abdel, Jouke-Jan Hottenga, Peter de Knijff, Michel G. Nivard, Xiangjun Xiao, Paul Scheet, Andrew Brooks, et al. 2013. "Population Structure, Migration, and Diversifying Selection in the Netherlands." European Journal of Human Genetics: EJHG 21 (11): 127785.

Acemoglu, Daron. 2002. "Technical Change, Inequality, and the Labor Market.” Journal of Economic Literature 40 (1): 7-72.

Altshuler, David M., Richard A. Gibbs, and Leena Peltonen. 2010. "Integrating Common and Rare Genetic Variation in Diverse Human Populations.” Nature 467 (7311): 52-58.

Auton, Adam, Goncalo R. Abecasis, David M. Altshuler, Richard M. Durbin, David R. Bentley, Aravinda Chakravarti, Andrew G. Clark, et al. 2015. "Genetic Variance Estimation with Imputed Variants Finds Negligible Missing Heritability for Human Height and Body Mass Index." Nature Genetics 526 (7571): 1114-20.

Autor, David H. 2001. "Wiring the Labor Market." The Journal of Economic Perspectives: A Journal of the American Economic Association 15 (1): 25-40.

Barth, Daniel, Nicholas W. Papageorge, and Kevin Thom. 2020. "Genetic Endowments and Wealth Inequality." The Journal of Political Economy, no. 24642. https://doi.org/10.3386/w24642.

Becker, Gary S. 1965. "A Theory of the Allocation of Time.” The Economic Journal 75 (299): 493-517.

Benjamin, Daniel J., David Cesarini, Matthijs J. H. M. van der Loos, Christopher T. Dawes, Philipp D. Koellinger, Patrik K. E. Magnusson, Christopher F. Chabris, et al. 2012. "The Genetic Architecture of Economic and Political Preferences." Proceedings of the National Academy of Sciences of the United States of America 109 (21): 8026-31.

Benjamin, D. J., D. Cesarini, C. F. Chabris, E. L. Glaeser, D. I. Laibson, V. Gudnason, T. B. Harris, et al. 2012. "The Promises and Pitfalls of Genoeconomics." Annual Review of Economics 4 (1): 627-62.

Bertrand, Marianne, and Sendhil Mullainathan. 2004. “Are Emily and Greg More Employable than Lakisha and Jamal? A Field Experiment on Labor Market Discrimination." The American Economic Review 94 (4): 991-1013.

Bulik-Sullivan, Brendan, Hilary K. Finucane, Verneri Anttila, Alexander Gusev, Felix R. Day, Reprogen Consortium, Psychiatric Genomics Consortium, et al. 2015. "An Atlas of Genetic Correlations across Human Diseases and Traits." Nature Genetics 47 (11): 1236-41.

Bulik-Sullivan, Brendan K., Po-Ru Loh, Hilary K. Finucane, Stephan Ripke, Jian Yang, Schizophrenia Working Group of the Psychiatric Genomics Consortium, Nick Patterson, Mark J. Daly, Alkes L. Price, and Benjamin M. Neale. 2015. "LD Score Regression Distinguishes Confounding from Polygenicity in Genome-Wide Association Studies." Nature Genetics 47 (3): 291-95.

Buniello, Annalisa, Jacqueline A. L. MacArthur, Maria Cerezo, Laura W. Harris, James Hayhurst, Cinzia Malangone, Aoife McMahon, et al. 2019. "The NHGRI-EBI GWAS Catalog of Published Genome-Wide Association Studies, Targeted Arrays and Summary Statistics 2019." Nucleic Acids Research 47 (D1): D1005-12.

Bycroft, Clare, Colin Freeman, Desislava Petkova, Gavin Band, Lloyd T. Elliott, Kevin Sharp, Allan Motyer, et al. 2018. "The UK Biobank Resource with Deep Phenotyping and Genomic Data." Nature 562 (7726): 203-9. 
Canela-Xandri, Oriol, Konrad Rawlik, and Albert Tenesa. 2018. "An Atlas of Genetic Associations in UK Biobank." Nature Genetics 50 (11): 1593-99.

Chabris, Christopher F., James J. Lee, David Cesarini, Daniel J. Benjamin, and David I. Laibson. 2015. "The Fourth Law of Behavior Genetics." Current Directions in Psychological Science 24 (4): 304-12.

Chetty, Raj, Michael Stepner, Sarah Abraham, Shelby Lin, Benjamin Scuderi, Nicholas Turner, Augustin Bergeron, and David Cutler. 2016. "The Association between Income and Life Expectancy in the United States, 2001-2014." JAMA: The Journal of the American Medical Association 315 (16): 1750.

Consortium, The 1000 Genomes Project, and The 1000 Genomes Project Consortium. 2012. "An Integrated Map of Genetic Variation from 1,092 Human Genomes." Nature. https://doi.org/10.1038/nature11632.

Daetwyler, H. D., B. Villanueva, and J. A. Woolliams. 2008. "Accuracy of Predicting the Genetic Risk of Disease Using a Genome-Wide Approach.” PloS One 3 (10): e3395.

Evans, Luke M., Rasool Tahmasbi, Scott I. Vrieze, Gonçalo R. Abecasis, Sayantan Das, Steven Gazal, Douglas W. Bjelland, et al. 2018. "Comparison of Methods That Use Whole Genome Data to Estimate the Heritability and Genetic Architecture of Complex Traits." Nature Genetics 50 (5): 737-45.

Gibson, Greg. 2012. "Rare and Common Variants: Twenty Arguments." Nature Reviews. Genetics 13 (2): 135-45.

Hamer, Dean H., and L. Sirota. 2000. "Beware the Chopsticks Gene.” Molecular Psychiatry 5 (1): 11-13.

Harden, K. P., and P. D. Koellinger. 2020. "Using Genetics for Social Science.” Nature Human Behaviour. https://doi.org/10.1038/s41562-020-0862-5.

Haworth, Simon, Ruth Mitchell, Laura Corbin, Kaitlin H. Wade, Tom Dudding, Ashley BuduAggrey, David Carslake, et al. 2019. "Apparent Latent Structure within the UK Biobank Sample Has Implications for Epidemiological Analysis.” Nature Communications 10 (1): 333.

Hemani, Gibran, Jack Bowden, and George Davey Smith. 2018. "Evaluating the Potential Role of Pleiotropy in Mendelian Randomization Studies." Human Molecular Genetics 27 (R2): R195-208.

Herd, Pamela. 2016. "Quality Control Report for Genotypic Data." University of Washington. https://www.ssc.wisc.edu/wlsresearch/documentation/GWAS/Herd_QC_report.pdf.

Herd, Pamela, Deborah Carr, and Carol Roan. 2014. "Cohort Profile: Wisconsin Longitudinal Study (WLS).” International Journal of Epidemiology 43 (1): 34-41.

Hill, W. David, Neil M. Davies, Stuart J. Ritchie, Nathan G. Skene, Julien Bryois, Steven Bell, Emanuele Di Angelantonio, et al. 2019. "Genome-Wide Analysis Identifies Molecular Systems and 149 Genetic Loci Associated with Income.” Nature Communications 10 (1): 5741.

Hill, W. David, Saskia P. Hagenaars, Riccardo E. Marioni, Sarah E. Harris, David C. M. Liewald, Gail Davies, Aysu Okbay, Andrew M. McIntosh, Catharine R. Gale, and Ian J. Deary. 2016. "Molecular Genetic Contributions to Social Deprivation and Household Income in UK Biobank." Current Biology: CB 26 (22): 3083-89.

Hill, William G., Michael E. Goddard, and Peter M. Visscher. 2008. "Data and Theory Point to Mainly Additive Genetic Variance for Complex Traits.” Edited by Trudy F. C. Mackay. PLoS Genetics 4 (2): e1000008. 
HRS. 2013. "Imputation Report - 1000 Genomes Project Reference Panel." http://hrsonline.isr.umich.edu/sitedocs/genetics/1000G_IMPUTE2report_HRS2_2006_2008 2010.pdf.

Hyytinen, Ari, Pekka Ilmakunnas, Edvard Johansson, and Otto Toivanen. 2019. "Heritability of Lifetime Earnings." Journal of Economic Inequality 17 (3): 319-35.

Kaas, Leo, and Christian Manger. 2012. "Ethnic Discrimination in Germany's Labour Market: A Field Experiment." German Economic Review 13 (1): 1-20.

King, Emily A., J. Wade Davis, and Jacob F. Degner. 2019. "Are Drug Targets with Genetic Support Twice as Likely to Be Approved? Revised Estimates of the Impact of Genetic Support for Drug Mechanisms on the Probability of Drug Approval." PLoS Genetics 15 (12): e1008489.

Koellinger, P. D., and K. P. Harden. 2018. "Using Nature to Understand Nurture: Genetic Associations Show How Parenting Matters for Children's Education." Science 359 (6374). https://doi.org/10.1126/science.aar6429.

Koellinger, P. D., H. Kweon, C. Burik, T. DiPrete, R. Karlsson Linnér, and A. Okbay. 2018. "Genome-Wide Association Study on Income." Open Science Framework Registries. https://osf.io/rg8sh.

Kong, Augustine, Gudmar Thorleifsson, Michael L. Frigge, Bjarni J. Vilhjalmsson, Alexander I. Young, Thorgeir E. Thorgeirsson, Stefania Benonisdottir, et al. 2018. "The Nature of Nurture: Effects of Parental Genotypes." Science 359 (6374): 424-28.

Lee, James J., and Carson C. Chow. 2014. "Conditions for the Validity of SNP-Based Heritability Estimation." Human Genetics 133 (8): 1011-22.

Lee, James J., Matt McGue, William G. Iacono, and Carson C. Chow. 2018. "The Accuracy of LD Score Regression as an Estimator of Confounding and Genetic Correlations in GenomeWide Association Studies." Genetic Epidemiology 42 (8): 783-95.

Lee, James J., Robbee Wedow, Aysu Okbay, Edward Kong, Omeed Maghzian, Meghan Zacher, Tuan Anh Nguyen-Viet, et al. 2018. "Gene Discovery and Polygenic Prediction from a Genome-Wide Association Study of Educational Attainment in 1.1 Million Individuals." Nature Genetics 50 (8): 1112-21.

Loh, Po-Ru, George Tucker, Brendan K. Bulik-Sullivan, Bjarni J. Vilhjálmsson, Hilary K. Finucane, Rany M. Salem, Daniel I. Chasman, et al. 2015. "Efficient Bayesian MixedModel Analysis Increases Association Power in Large Cohorts." Nature Genetics 47 (3): 284-90.

Marchini, J., J. O’Connel, O. Delaneau, K. Sharp, W. Kretzschmar, G. Band, S. McCarthy, et al. 2015. "Genotype Imputation and Genetic Association Studies of UK Biobank." UK Biobank. http://www.ukbiobank.ac.uk/wpcontent/uploads/2014/04/imputation_documentation_May2015-1.pdf.

Martin, Alicia R., Masahiro Kanai, Yoichiro Kamatani, Yukinori Okada, Benjamin M. Neale, and Mark J. Daly. 2019. "Clinical Use of Current Polygenic Risk Scores May Exacerbate Health Disparities." Nature Genetics 51 (4): 584-91.

McCarthy, Shane, Sayantan Das, Warren Kretzschmar, Olivier Delaneau, Andrew R. Wood, Alexander Teumer, Hyun Min Kang, et al. 2016. "A Reference Panel of 64,976 Haplotypes for Genotype Imputation.” Nature Genetics 48 (10): 1279-83.

Mills, Melinda C., and Charles Rahal. 2019. "A Scientometric Review of Genome-Wide Association Studies." Communications Biology 2 (January): 9.

Mostafavi, Hakhamanesh, Arbel Harpak, Ipsita Agarwal, Dalton Conley, Jonathan K. Pritchard, 
and Molly Przeworski. 2020. "Variable Prediction Accuracy of Polygenic Scores within an Ancestry Group.” eLife 9 (January). https://doi.org/10.7554/eLife.48376.

Okbay, Aysu, Jonathan P. Beauchamp, Mark A. Fontana, James J. Lee, Tune H. Pers, Cornelius

A. Rietveld, Patrick Turley, et al. 2016. "Genome-Wide Association Study Identifies 74

Loci Associated with Educational Attainment." Nature 533: 539-42.

Palmer, Cameron, and Itsik Pe'er. 2017. "Statistical Correction of the Winner's Curse Explains

Replication Variability in Quantitative Trait Genome-Wide Association Studies.” PLoS

Genetics 13 (7): e1006916.

Papageorge, Nicholas W., and Kevin Thom. 2019. "Genes, Education, and Labor Market Outcomes: Evidence from the Health and Retirement Study." Journal of the European

Economic Association, December. https://doi.org/10.1093/jeea/jvz072.

Piotrowska, Patrycja J., Christopher B. Stride, Simone E. Croft, and Richard Rowe. 2015.

"Socioeconomic Status and Antisocial Behaviour among Children and Adolescents: A

Systematic Review and Meta-Analysis." Clinical Psychology Review 35 (February): 47-55.

Plomin, Robert. 2019. Blueprint: How DNA Makes Us Who We Are. MIT Press.

Price, Alkes L., Nick J. Patterson, Robert M. Plenge, Michael E. Weinblatt, Nancy A. Shadick, and David Reich. 2006. "Principal Components Analysis Corrects for Stratification in Genome-Wide Association Studies." Nature Genetics 38 (8): 904-9.

Purcell, Shaun, Benjamin Neale, Kathe Todd-Brown, Lori Thomas, Manuel A. R. Ferreira, David Bender, Julian Maller, et al. 2007. "PLINK: A Tool Set for Whole-Genome Association and Population-Based Linkage Analyses." The American Journal of Human Genetics. https://doi.org/10.1086/519795.

Reimers, Cordelia W. 1983. "Labor Market Discrimination against Hispanic and Black Men." The Review of Economics and Statistics 65 (4): 570-79.

Rietveld, Cornelius A., Sarah E. Medland, Jaime Derringer, Jian Yang, Tõnu Esko, Nicolas W. Martin, Harm-Jan Westra, et al. 2013. "GWAS of 126,559 Individuals Identifies Genetic Variants Associated with Educational Attainment." Science 340 (6139): 1467-71.

Rosenberg, Noah A., Michael D. Edge, Jonathan K. Pritchard, and Marcus W. Feldman. 2019. "Interpreting Polygenic Scores, Polygenic Adaptation, and Human Phenotypic Differences." Evolution, Medicine, and Public Health 2019 (1): 26-34.

Shavers-Hornaday, Vickie L., Charles F. Lynch, Leon F. Burmeister, and James C. Torner. 1997. "Why Are African Americans Under-represented in Medical Research Studies?

Impediments to Participation." Ethnicity \& Health 2 (1-2): 31-45.

Siebert, Horst. 1997. "Labor Market Rigidities: At the Root of Unemployment in Europe." The Journal of Economic Perspectives: A Journal of the American Economic Association 11 (3): 37-54.

Solovieff, Nadia, Chris Cotsapas, Phil H. Lee, Shaun M. Purcell, and Jordan W. Smoller. 2013. "Pleiotropy in Complex Traits: Challenges and Strategies." Nature Reviews. Genetics 14 (7): 483-95.

Stringhini, Silvia, Cristian Carmeli, Markus Jokela, Mauricio Avendaño, Peter Muennig, Florence Guida, Fulvio Ricceri, et al. 2017. "Socioeconomic Status and the $25 \times 25$ Risk Factors as Determinants of Premature Mortality: A Multicohort Study and Meta-Analysis of 1 \textperiodcentered 7 Million Men and Women." The Lancet 389 (10075): 1229-37.

Taubman, Paul. 1976a. "Earnings, Education, Genetics, and Environment." The Journal of Human Resources 11 (4): 447-61.

. 1976b. "The Determinants of Earnings: Genetics, Family, and Other Environments: A 
Study of White Male Twins." The American Economic Review 66 (5): 858-70.

Turley, Patrick, Raymond K. Walters, Omeed Maghzian, Aysu Okbay, James J. Lee, Mark Alan Fontana, Tuan Anh Nguyen-Viet, et al. 2018. "Multi-Trait Analysis of Genome-Wide Association Summary Statistics Using MTAG." Nature Genetics 50 (2): 229-37.

Vilhjálmsson, Bjarni J., Jian Yang, Hilary K. Finucane, Alexander Gusev, Sara Lindström, Stephan Ripke, Giulio Genovese, et al. 2015. "Modeling Linkage Disequilibrium Increases Accuracy of Polygenic Risk Scores." American Journal of Human Genetics 97 (4): 576-92. Visscher, Peter M. 2010. "A Commentary on Common SNPs Explain a Large Proportion of the Heritability for Human Height by Yang et Al." Twin Research and Human Genetics: The Official Journal of the International Society for Twin Studies 13 (6): 517.

Visscher, Peter M., Naomi R. Wray, Qian Zhang, Pamela Sklar, Mark I. McCarthy, Matthew A. Brown, and Jian Yang. 2017. "10 Years of GWAS Discovery: Biology, Function, and Translation." American Journal of Human Genetics 101 (1): 5-22.

Vlaming, Ronald de, Aysu Okbay, Cornelius A. Rietveld, Magnus Johannesson, Patrik K. E. Magnusson, André G. Uitterlinden, Frank J. A. van Rooij, et al. 2017. "Meta-GWAS Accuracy and Power (MetaGAP) Calculator Shows That Hiding Heritability Is Partially due to Imperfect Genetic Correlations across Studies." PLoS Genetics 13 (1). https://doi.org/10.1371/journal.pgen.1006495.

Wainschtein, Pierrick, Deepti P. Jain, Loic Yengo, Zhili Zheng, TOPMed Anthropometry Working Group, Trans-Omics for Precision Medicine Consortium, L. Adrienne Cupples, Aladdin H. Shadyab, et al. 2019. "Recovery of Trait Heritability from Whole Genome Sequence Data." bioRxiv. https://doi.org/10.1101/588020.

Watanabe, Kyoko, Erdogan Taskesen, Arjen van Bochoven, and Danielle Posthuma. 2017. "Functional Mapping and Annotation of Genetic Associations with FUMA." Nature Communications 8 (1): 1826.

Weir, David. 2013. "Quality Control Report for Genotypic Data.” September 4, 2013. http://hrsonline.isr.umich.edu/sitedocs/genetics/HRS2_qc_report_SEPT2013.pdf.

Wilkinson, Richard G., and Michael Marmot. 2003. Social Determinants of Health: The Solid Facts. World Health Organization.

Witte, John S., Peter M. Visscher, and Naomi R. Wray. 2014. "The Contribution of Genetic Variants to Disease Depends on the Ruler." Nature Reviews. Genetics 15 (11): 765-76.

WLS. 2016. "Imputation Report."

https://www.ssc.wisc.edu/wlsresearch/documentation/GWAS/Herd_1000G_IMPUTE2repor t.pdf.

Yang, Jian, Beben Benyamin, Brian P. McEvoy, Scott Gordon, Anjali K. Henders, Dale R. Nyholt, Pamela A. Madden, et al. 2010. "Common SNPs Explain a Large Proportion of the Heritability for Human Height." Nature Genetics 42 (7): 565-69.

Yang, Jian, Noah A. Zaitlen, Michael E. Goddard, Peter M. Visscher, and Alkes L. Price. 2014. "Advantages and Pitfalls in the Application of Mixed-Model Association Methods." Nature Genetics 46 (2): 100-106.

Yang, Jian, Jian Zeng, Michael E. Goddard, Naomi R. Wray, and Peter M. Visscher. 2017. "Concepts, Estimation and Interpretation of SNP-Based Heritability." Nature Genetics 49 (9): 1304-10.

Young, Alexander I., Stefania Benonisdottir, Molly Przeworski, and Augustine Kong. 2019. "Deconstructing the Sources of Genotype-Phenotype Associations in Humans." Science 365 (6460): 1396-1400. 
Young, Alexander I., Michael L. Frigge, Daniel F. Gudbjartsson, Gudmar Thorleifsson, Gyda Bjornsdottir, Patrick Sulem, Gisli Masson, Unnur Thorsteinsdottir, Kari Stefansson, and Augustine Kong. 2018. "Relatedness Disequilibrium Regression Estimates Heritability without Environmental Bias." Nature Genetics 50 (9): 1304-10. 
Table A1. List of variables in the UK Biobank

\begin{tabular}{|c|c|}
\hline Variables & Details \\
\hline log hourly wage & Imputed from standard occupation codes. See Appendix \\
\hline top household income & $=1$ if the annual household income before tax is greater than $£ 52,000$. \\
\hline log regional income & $\begin{array}{l}\text { Computed by matching home locations to Middle-layer Super Output Areas. Data from } \\
\text { Official National Statistics (England and Wales only) }\end{array}$ \\
\hline neighborhood score & $\begin{array}{l}\text { Negative of index of multiple deprivation for Lower-layer Super Output Areas derived b. } \\
\text { the UK's Ministry of Housing, Communities \& Local Government (England only) }\end{array}$ \\
\hline years of education & See Okbay et al. (2016) \\
\hline college degree & $=1$ if having a college degree \\
\hline waist-to-hip ratio & Waist circumference divided by hip circumference \\
\hline BMI & Body mass index \\
\hline blood pressure & Average of systolic and diastolic measures \\
\hline lung function & $\begin{array}{l}\text { Average of forced expiratory volume in the first second, forced vital capacity, and peak } \\
\text { expiratory flow weighted by their covariance matrix. See Barcellos et al. (2019). }\end{array}$ \\
\hline ever hospitalized & $=1$ if any record in in-patient data \\
\hline ever diagnosed with cancer & $=1$ if any record in cancer registry \\
\hline infectious and parasitic diseases & $=1$ if any record for disorders classified in ICD-10 chapter 1 and codes Z20-29 \\
\hline neoplasms & $=1$ if any record for disorders classified in ICD-10 chapter 2 \\
\hline diseases of blood organs and immune system & $=1$ if any record for disorders classified in ICD-10 chapter 3 \\
\hline $\begin{array}{l}\text { endocrine, nutritional, and metabolic } \\
\text { diseases }\end{array}$ & $=1$ if any record for disorders classified in ICD-10 chapter 4 \\
\hline mental, behavioral, nervous system disorders & $=1$ if any record for disorders classified in ICD-10 chapter 5 and 6 \\
\hline diseases of the eye and adnexa & $=1$ if any record for disorders classified in ICD-10 chapter 7 \\
\hline diseases of the circulatory system & $=1$ if any record for disorders classified in ICD-10 chapter 9 \\
\hline diseases of the respiratory system & $=1$ if any record for disorders classified in ICD-10 chapter 10 \\
\hline
\end{tabular}


diseases of the skin and subcutaneous tissue

diseases of musculoskeletal system and connective tissue

diseases of genitourinary system

symptoms and signs not elsewhere classified

injury, poisoning, and other consequences of external causes

external causes of morbidity and mortality

other health conditions
$=1$ if any record for disorders classified in ICD-10 chapter 12

$=1$ if any record for disorders classified in ICD-10 chapter 13

$=1$ if any record for disorders classified in ICD-10 chapter 14

$=1$ if any record for disorders classified in ICD-10 chapter 18

$=1$ if any record for disorders classified in ICD-10 chapter 19

$=1$ if any record for disorders classified in ICD-10 chapter 20

$=1$ if any record for the rest of disorders classified in ICD-10 
Table A2. Descriptive statistics

\begin{tabular}{|c|c|c|c|c|c|}
\hline & $\mathbf{N}$ & Mean & S.D & Min & Max \\
\hline \multicolumn{6}{|l|}{ UK Biobank } \\
\hline year of birth & 38697 & 1951.045 & 7.347 & 1937 & 1969 \\
\hline male & 38697 & 0.421 & 0.494 & 0 & 1 \\
\hline log hourly wage & 25292 & 2.6 & 0.349 & 1.833 & 3.881 \\
\hline top household income & 33477 & 0.233 & 0.423 & 0 & 1 \\
\hline regional income & 35393 & 739.482 & 193.387 & 300 & 1730 \\
\hline neighborhood score & 33276 & -17.646 & 13.937 & -82 & -0.61 \\
\hline years of education & 38424 & 14.627 & 5.135 & 7 & 20 \\
\hline college degree & 38424 & 0.291 & 0.454 & 0 & 1 \\
\hline waist-to-hip ratio & 38638 & 0.866 & 0.089 & 0.581 & 1.342 \\
\hline BMI & 38596 & 27.267 & 4.706 & 13.789 & 63.809 \\
\hline blood pressure & 36099 & 110.879 & 13.898 & 65.5 & 179 \\
\hline lung function & 35357 & 0 & 0.849 & -3.922 & 20.781 \\
\hline ever hospitalized & 38697 & 0.831 & 0.375 & 0 & 1 \\
\hline ever diagnosed with cancer & 38697 & 0.183 & 0.387 & 0 & 1 \\
\hline infectious and parasitic diseases & 38697 & 0.098 & 0.297 & 0 & 1 \\
\hline neoplasms & 38697 & 0.176 & 0.381 & 0 & 1 \\
\hline diseases of blood organs and immune system & 38697 & 0.222 & 0.416 & 0 & 1 \\
\hline endocrine, nutritional, and metabolic diseases & 38697 & 0.216 & 0.412 & 0 & 1 \\
\hline mental, behavioral, nervous system disorders & 38697 & 0.186 & 0.389 & 0 & 1 \\
\hline diseases of the eye and adnexa & 38697 & 0.117 & 0.322 & 0 & 1 \\
\hline diseases of the circulatory system & 38697 & 0.359 & 0.48 & 0 & 1 \\
\hline diseases of the respiratory system & 38697 & 0.172 & 0.378 & 0 & 1 \\
\hline diseases of the digestive system & 38697 & 0.426 & 0.494 & 0 & 1 \\
\hline diseases of the skin and subcutaneous tissue & 38697 & 0.118 & 0.323 & 0 & 1 \\
\hline diseases of musculoskeletal system and connective tissue & 38697 & 0.316 & 0.465 & 0 & 1 \\
\hline diseases of genitourinary system & 38697 & 0.283 & 0.45 & 0 & 1 \\
\hline symptoms and signs not elsewhere classified & 38697 & 0.389 & 0.487 & 0 & 1 \\
\hline injury, poisoning, and other consequences of external causes & 38697 & 0.171 & 0.376 & 0 & 1 \\
\hline external causes of morbidity and mortality & 38697 & 0.181 & 0.385 & 0 & 1 \\
\hline other health conditions & 38697 & 0.494 & 0.5 & 0 & 1 \\
\hline \multicolumn{6}{|l|}{ Health and Retirement Study } \\
\hline number of survey responses & 6171 & 3.605 & 2.047 & 1 & 12 \\
\hline year of birth & 6171 & 1944.487 & 8.431 & 1928 & 1968 \\
\hline male & 6171 & 0.451 & 0.498 & 0 & 1 \\
\hline hourly earnings & 22247 & 11.622 & 15.908 & 0.002 & 1244.848 \\
\hline
\end{tabular}


Table A2. Descriptive statistics

\begin{tabular}{lccccc}
\hline & N & Mean & S.D & Min & Max \\
\hline job experience & 22180 & 36.37 & 5.531 & 7 & 57 \\
years of education & 6152 & 13.588 & 2.414 & 0 & 17 \\
college degree & 6152 & 0.307 & 0.461 & 0 & 1 \\
father's years of education & 5635 & 10.667 & 3.538 & 0 & 17 \\
mother's yeras of education & 5862 & 11.06 & 2.918 & 0 & 17 \\
Wisconsin Longitudinal Study & & & & & \\
year of birth & 7387 & 39.742 & 3.747 & 29 & 64 \\
male & 7387 & 0.494 & 0.5 & 0 & 1 \\
sibling respondent & 7387 & 0.319 & 0.466 & 0 & 1 \\
hourly earnings & 7387 & 18.261 & 33.418 & 0.04 & 1282.051 \\
years of education & 6902 & 13.905 & 2.423 & 7 & 21 \\
\hline
\end{tabular}


Table A3. Summary of the UK Biobank samples

\begin{tabular}{|c|c|c|c|}
\hline Analysis & Sample & $\mathbf{N}$ & Note \\
\hline \multirow[t]{2}{*}{ Heritability } & Single-variance-parameter model & 196,187 & unrelated, wage observed \\
\hline & Multiple-variance-parameter model & 24,000 & randomly selected, unrelated, wage observed \\
\hline \multirow[t]{4}{*}{ GWAS } & Baseline sample & 282,963 & occupational wage observed \\
\hline & Two sub-samples & 141,481 each & $\begin{array}{l}\text { for GIV prediction, occupational wage observed, no relatives across the sub- } \\
\text { samples }\end{array}$ \\
\hline & Baseline sample without siblings and their relative & 252,958 & for prediction with the sibling sample, wage observed \\
\hline & Two sub-samples without siblings and their relative & 126,478 each & $\begin{array}{l}\text { for GIV prediction with the sibling sample, wage observed, no relatives across } \\
\text { the sub-samples }\end{array}$ \\
\hline Prediction & Sibling sample & 38,698 & 18,807 genetic sibling groups for within-family prediction analyses \\
\hline
\end{tabular}


Table A4. Weights used to construct the summary index of health in the UK

Biobank sample

\begin{tabular}{lc}
\hline Health measure & Weight \\
\hline waist-to-hip ratio & 0.070 \\
BMI & 0.055 \\
blood pressure & 0.113 \\
lung function & 0.079 \\
ever hospitalized & 0.001 \\
ever diagnosed with cancer & 0.049 \\
infectious and parasitic diseases & 0.050 \\
neoplasms & 0.037 \\
diseases of blood organs and immune system & 0.062 \\
endocrine, nutritional, and metabolic diseases & -0.003 \\
mental, behavioral, nervous system disorders & 0.050 \\
diseases of the eye and adnexa & 0.101 \\
diseases of the circulatory system & -0.026 \\
diseases of the respiratory system & 0.041 \\
diseases of the digestive system & 0.044 \\
diseases of the skin and subcutaneous tissue & 0.080 \\
diseases of musculoskeletal system and connective tissue & 0.042 \\
diseases of genitourinary system & 0.077 \\
symptoms and signs not elsewhere classified & 0.019 \\
injury, poisoning, and other consequences of external causes & 0.065 \\
external causes of morbidity and mortality & 0.010 \\
other health conditions & -0.016 \\
\hline
\end{tabular}


Table A5. Heritability estimation of occupational wages in the UK Biobank using a single variance genetic relatedness matrix restricted maximum likelihood (GREML)

\begin{tabular}{lcc}
\hline & Estimates & $N$ \\
\hline Male+Female & $0.103(0.003)$ & 196,187 \\
Male & $0.103(0.005)$ & 93,666 \\
Female & $0.126(0.005)$ & 102,521 \\
\hline
\end{tabular}

Note: Standard errors in parentheses. 
Table A6. Lead single nucleotide polymorphisms (SNPs) from a genome-wide association study (GWAS) on log occupational wages in the UK Biobank and overlap with other traits.

\begin{tabular}{|c|c|c|c|c|c|}
\hline SNP ID & $\begin{array}{l}\text { Chrom } \\
\text { o-some }\end{array}$ & $\begin{array}{l}\text { Effect- } \\
\text { coded } \\
\text { allele }\end{array}$ & $\begin{array}{c}\text { Effect } \\
\text { allele } \\
\text { frequency }\end{array}$ & Beta $\quad R^{2}(\%)$ & Overlap with other traits \\
\hline
\end{tabular}

rs148744

6

0.485

0.0097

Alcohol consumption (drinks per week), Autism and educational attainment, Bipolar disorder, Cognitive ability, Educational attainment, Extremely high intelligence, General risk tolerance, Highest math class taken, Household income, Intelligence, QT interval (drug interaction), Regular attendance at a pub or social club, Risk-taking tendency (4domain principal component model), Self-reported math ability, Tourette syndrome

Blood protein levels, Chronic inflammatory diseases (ankylosing spondylitis, Crohn's disease, psoriasis, primary sclerosing cholangitis, ulcerative colitis) (pleiotropy), Cognitive ability, Crohn's disease, Depressed affect, Educational attainment, Estimated glomerular filtration rate, Extremely high intelligence, Feeling fed-up, Glioblastoma, Glioma, Gut microbiota (functional units), Highest math class taken, Household income, Inflammatory bowel disease, Intelligence, Menarche (age at onset), Metabolite levels, Parental longevity (father's age at death or father's attained age), Pediatric autoimmune diseases, Primary sclerosing cholangitis, Regular attendance at a religious group, Self-reported math ability, Ulcerative colitis

BMI, Cognitive ability, Cognitive ability, years of educational attainment or schizophrenia (pleiotropy), Depressed affect, Educational attainment, Feeling miserable, Gastroesophageal reflux disease, HDL cholesterol, HDL cholesterol levels, HDL cholesterol levels in current drinkers, HDL cholesterol levels x alcohol consumption (drinkers vs nondrinkers) interaction (2df), HDL cholesterol levels x alcohol consumption (regular vs non-regular drinkers) interaction (2df), HDL cholesterol x physical activity interaction (2df test), Household income, Intelligence, Menarche (age at onset), Morning person, Morningness, Parental longevity (mother's age at death or mother's attained age), Predicted visceral adipose tissue, Regular attendance at a gym or sports club, White blood cell count

Balding type 1, BMI, Cognitive ability, Household income, Intelligence, Male-pattern baldness

Autism and educational attainment, Bipolar disorder, Cognitive ability, Depressed affect, Educational attainment, Extremely high intelligence, Feeling lonely, Highest math class taken, Household income, Insomnia symptoms (never/rarely vs. sometimes/usually), Insomnia symptoms (never/rarely vs. usually), Intelligence, Mental health study participation (completed survey), Remission after SSRI treatment in MDD or neuroticism, Self-reported math ability

Cognitive ability, Depression, Educational attainment, Highest math class taken, Household income, Intelligence, Lung function (FVC), Major depressive disorder, Sedentary behaviour duration, Self-reported math ability

Cognitive ability, Cognitive ability, years of educational attainment or schizophrenia (pleiotropy), Depressed affect, Depression, Depression (broad), Educational attainment, Experiencing mood swings, Feeling fed-up, Feeling hurt, General factor of neuroticism, Highest math class taken, Household income, Lifetime smoking index, Mood instability, Neuroticism, Neuroticism, Remission after SSRI treatment in MDD or neuroticism, Response to amphetamines, Subjective well-being, Well-being spectrum (multivariate analysis), Worry

Alcohol consumption (drinks per week), Alzheimer's disease in APOE e4- carriers, Balding type 1, Brain region volumes, Breast cancer, Celiac disease, Cognitive ability, Depressed affect, Epithelial ovarian cancer, Experiencing mood swings, Feeling fed-up, Feeling guilty, Feeling hurt, Feeling miserable, Feeling nervous, Feeling worry, General

Handedness (non-right-handed vs right-handed), Intelligence, Intracranial volume, Intraocular pressure, Irritable mood, Lung function (FEV1), Lung function (FVC), Macular thickness, Male-pattern baldness, Multiple system atrophy, Neuroticism, Neuroticism, Ovarian cancer in BRCA1 mutation carriers, Parkinson's disease, Parkinson's disease or first 
degree relation to individual with Parkinson's disease, Post bronchodilator FEV1, Sense of smell, White matter microstructure (axial diusivities), White matter microstructure (fractional anisotropy), White matter microstructure (mean diusivities), White matter microstructure (radial diusivities), Worry

BMI, General risk tolerance, Household income, Predicted visceral adipose tissue, Risk-taking tendency (4-domain principal component model), Smoking initiation (ever regular vs never regular), Smoking status, Smoking status (ever vs never smokers), Waist circumference, Waist-hip ratio

Albumin-globulin ratio, Alcohol consumption (drinks per week), Allergic disease (asthma, hay fever or eczema), Bipolar disorder or body mass index, Blood protein levels, BMI, BMI (joint analysis main effects and physical activity interaction), BMI in physically active individuals, Body fat percentage, Childhood obesity, Chronic inflammatory diseases (ankylosing spondylitis, Crohn's disease, psoriasis, primary sclerosing cholangitis, ulcerative colitis)

(pleiotropy), Chronic obstructive pulmonary disease, Cognitive ability, Crohn's disease, Eczema, Educationa

attainment, Estimated glomerular filtration rate, Extremely high intelligence, Hand grip strength, Hip circumference, Hip circumference adjusted for BMI, Household income, Inflammatory bowel disease, Inflammatory bowel disease (early onset), Intelligence, Offspring birth weight, Pediatric autoimmune diseases, Red blood cell count, Red cell distribution width, Type 1 diabetes, Ulcerative colitis, Waist circumference, Weight

Alcohol consumption (drinks per week), Cognitive ability, Cognitive ability, years of educational attainment or

rs 10053567

Educational attainment, Height, Highest math class taken, Household income

rs619466

Educational attainment

rs 3847223

Cognitive ability, Depressed affect, Educational attainment, Highest math class taken, Household income, Intelligence, Self-reported math ability

rs59971723

Educational attainment, Household income

\section{Educational attainment}

Cognitive ability, Cognitive ability, years of educational attainment or schizophrenia (pleiotropy), Educational attainment, Height, Highest math class taken, Household income, Intelligence

Cognitive ability, Household income, Intelligence

Adventurousness, Alcohol consumption, Alcohol consumption (drinks per week), Alcohol use disorder, Alcohol use disorder (consumption score), Alcohol use disorder (dependence and problematic use scores), Alcohol use disorder (total score), Autism spectrum disorder or schizophrenia, Balding type 1, Bitter alcoholic beverage consumption, Blood pressure, BMI, BMI (adjusted for smoking behaviour), BMI (joint analysis main effects and physical activity interaction), BMI (joint analysis main effects and smoking interaction), BMI in non-smokers, BMI in physically active individuals, Body fat percentage, Brain imaging measurements, Brain region volumes, Childhood body mass index, Cognitive ability, Cognitive ability, years of educational attainment or schizophrenia (pleiotropy), Diastolic blood pressure, Diastolic blood pressure $\mathrm{x}$ alcohol consumption interaction (2df test), Eczema, Educational attainment, Hand grip strength, HDL cholesterol, HDL cholesterol levels, HDL cholesterol levels in current drinkers, HDL cholesterol levels $\mathrm{x}$ alcohol consumption (drinkers vs non-drinkers) interaction (2df), HDL cholesterol levels $\mathrm{x}$ alcohol consumption 
(regular vs non-regular drinkers) interaction (2df), HDL cholesterol x physical activity interaction (2df test), Height, High density lipoprotein cholesterol levels, Hypertension, Insomnia, Intelligence, Low density lipoprotein cholesterol levels, Lung function (FVC), Male-pattern baldness, Mean arterial pressure, Mean arterial pressure x alcohol

consumption interaction (2df test), Medication use (agents acting on the renin-angiotensin system), Multisite chronic pain, NT-proBNP levels in acute coronary syndrome, Obese vs. thin, Osteoarthritis, Predicted visceral adipose tissue, Red blood cell count, Risk-taking tendency (4-domain principal component model), Schizophrenia, Self-reported math ability, Sleep duration (short sleep), Systolic blood pressure, Systolic blood pressure x alcohol consumption interaction (2df test), Total cholesterol levels, Voxel-wise structural brain imaging measurements, vWF levels, Waist-hip ratio, Waist-to-hip ratio adjusted for BMI, White blood cell count

rs10818605

$0.511 \quad 0.0053$

\section{Household income, Multiple system atrophy}

Age of smoking initiation, Birth weight, Chronotype, Educational attainment, Height, Lifetime smoking index,

0.012 Mosquito bite size, Offspring birth weight, Parental longevity (father's age at death or father's attained age), Smoking cessation

\subsection{Educational attainment, Household income}

Cognitive ability, Educational attainment, Experiencing mood swings, Highest math class taken, Household income, Inflammatory bowel disease, Intelligence, Mood instability, Self-reported math ability, Smoking initiation (ever regular vs never regular), Smoking status, Ulcerative colitis

Carpal tunnel syndrome, Risk-taking tendency (4-domain principal component model), Smoking cessation, Waist-hip ratio

Chronotype, Educational attainment, Glaucoma, Glaucoma (high intraocular pressure), Glaucoma (primary open-angle), Heel bone mineral density, Height, Highest math class taken, Hip circumference adjusted for BMI, Medication use (antiglaucoma preparations and miotics), Menarche (age at onset), Optic cup area, Optic disc area, Optic disc size, Optic nerve measurement (rim area), Refractive error, Self-reported math ability, Vertical cup-disc ratio, Waist-hip ratio

$0.011 \quad$ Cognitive ability

BMI, Cognitive ability, Cognitive ability, years of educational attainment or schizophrenia (pleiotropy), Educational 0.011 attainment, Experiencing mood swings, Highest math class taken, Household income, Intelligence, Predicted visceral adipose tissue, Type 2 diabetes

Allergic rhinitis, Asthma and major depressive disorder, BMI, BMI (adjusted for smoking behaviour), BMI (joint analysis main effects and physical activity interaction), BMI (joint analysis main effects and smoking interaction), BMI in non-smokers, BMI in physically active individuals, BMI in physically inactive individuals, BMI in smokers, Body fat percentage, Childhood body mass index, Cognitive ability, Depression, Depression (broad), Depressive symptoms, Hip circumference, Insomnia, Intelligence, Major depressive disorder, Menarche (age at onset), Obesity, Obesity (early onset extreme), Subcutaneous adipose tissue, Waist circumference, Weight

\subsection{Educational attainment, Highest math class taken, Metabolite levels}

0.011 Intelligence 


\begin{tabular}{|c|c|c|c|c|c|c|}
\hline rs2726518 & 4 & $\mathrm{C}$ & 0.571 & -0.0053 & 0.011 & $\begin{array}{l}\text { Adventurousness, Cognitive ability, Colorectal cancer or advanced adenoma, Educational attainment, Highest math class } \\
\text { taken, Household income, Intelligence, Multiple sclerosis }\end{array}$ \\
\hline rs9824386 & 3 & A & 0.865 & 0.0077 & 0.011 & $\begin{array}{l}\text { Chronotype, Cognitive ability, Educational attainment, Highest math class taken, Intelligence, Metabolite levels, } \\
\text { Morningness }\end{array}$ \\
\hline rs7940022 & 11 & $\mathrm{~T}$ & 0.680 & 0.0056 & 0.011 & $\begin{array}{l}\text { Balding type 1, Cognitive ability, Cognitive ability, years of educational attainment or schizophrenia (pleiotropy), } \\
\text { Educational attainment, Highest math class taken, Household income, Intelligence, Male-pattern baldness }\end{array}$ \\
\hline rs12204714 & 6 & $\mathrm{~T}$ & 0.632 & 0.0054 & 0.011 & Age at first birth, Educational attainment \\
\hline rs2494995 & 1 & $\mathrm{C}$ & 0.217 & -0.0063 & 0.011 & $\begin{array}{l}\text { Adventurousness, Attention deficit hyperactivity disorder or cannabis use, Balding type } 1 \text {, Coffee consumption, } \\
\text { Cognitive ability, Educational attainment, Household income, Intelligence, Number of sexual partners, Perceived } \\
\text { intensity of sweet substances }\end{array}$ \\
\hline rs4953097 & 2 & $\mathrm{~T}$ & 0.663 & -0.0055 & 0.011 & Age at first sexual intercourse, Chronotype, Educational attainment, General risk tolerance, Height, Morning person \\
\hline rs9729959 & 1 & $\mathrm{~T}$ & 0.226 & 0.0062 & 0.011 & $\begin{array}{l}\text { Cognitive ability, Cognitive ability, years of educational attainment or schizophrenia (pleiotropy), Educational } \\
\text { attainment, Glioma, Highest math class taken, Household income, Intelligence, Non-glioblastoma glioma, Regular } \\
\text { attendance at a religious group, Self-reported math ability, Urinary sodium excretion }\end{array}$ \\
\hline rs13240401 & 7 & $\mathrm{C}$ & 0.224 & -0.0062 & 0.011 & Cognitive ability, Educational attainment, Highest math class taken, Self-reported math ability \\
\hline rs2282760 & 3 & G & 0.132 & -0.0076 & 0.011 & $\begin{array}{l}\text { Age at voice drop, Coffee consumption, Depressive symptom (appetite changes) (binary trait), Depressive symptom } \\
\text { (fatigue) (ordinal trait), Depressive symptoms (binary sum-score), Depressive symptoms (sum-score), Diastolic blood } \\
\text { pressure, Eosinophil counts, Lung function (FVC), Morningness, Systolic blood pressure, Waist-to-hip ratio adjusted for } \\
\text { BMI (additive genetic model) }\end{array}$ \\
\hline rs 141349367 & 8 & $\mathrm{~T}$ & 0.059 & -0.0109 & 0.011 & \\
\hline rs4408596 & 17 & $\mathrm{G}$ & 0.623 & 0.0053 & 0.011 & Cognitive ability, Intelligence, Morning person \\
\hline
\end{tabular}

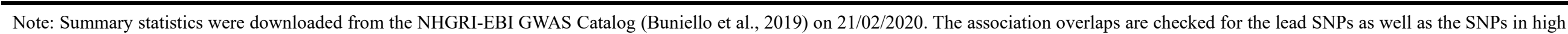

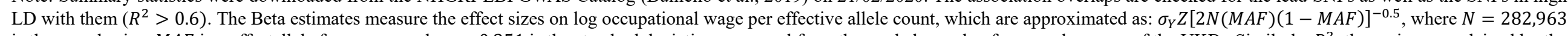

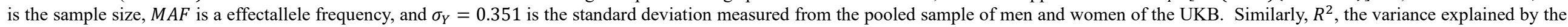
SNP alone, is approximated as $Z^{2} / N$. 
Table A7. Associations between polygenic indices for income and self-reported wages in the Health and Retirement Study and the Wisconsin Longitudinal Study

\begin{tabular}{|c|c|c|c|c|c|c|c|c|c|c|c|c|}
\hline \multirow{2}{*}{\multicolumn{3}{|c|}{ Panel A: Health and Retirement Study }} & \multicolumn{2}{|c|}{ Iale } & \multicolumn{4}{|c|}{ Female } & \multicolumn{4}{|c|}{ Male+Female } \\
\hline & & & & & & & & & & & & \\
\hline Income PGI & & $\begin{array}{l}0.083 * * * \\
(0.014)\end{array}$ & & $\begin{array}{c}0.047 * * \\
(0.017)\end{array}$ & & $\begin{array}{l}0.078 * * * \\
(0.011)\end{array}$ & & $\begin{array}{c}0.043 * * \\
(0.014)\end{array}$ & & $\begin{array}{c}0.080 * * * \\
(0.009)\end{array}$ & & $\begin{array}{l}0.045 * * * \\
(0.011)\end{array}$ \\
\hline EA PGI & & & $\begin{array}{l}0.093 * * * \\
(0.013)\end{array}$ & $\begin{array}{l}0.065 * * * \\
(0.017)\end{array}$ & & & $\begin{array}{l}0.089 * * * \\
(0.011)\end{array}$ & $\begin{array}{l}0.065 * * * \\
(0.014)\end{array}$ & & & $\begin{array}{l}0.091 * * * \\
(0.009)\end{array}$ & $\begin{array}{l}0.065 * * * \\
(0.011)\end{array}$ \\
\hline$R^{2}(\%)$ & 2.201 & 3.192 & 3.368 & 3.585 & 4.152 & 5.086 & 5.304 & 5.509 & 8.625 & 9.531 & 9.720 & 9.919 \\
\hline$\Delta R^{2}(\%)$ & & 0.991 & 1.167 & 1.384 & & 0.934 & 1.152 & 1.358 & & 0.905 & 1.095 & 1.294 \\
\hline partial $R^{2}(\%)$ & & 1.013 & 1.194 & 1.415 & & 0.975 & 1.202 & 1.416 & & 0.991 & 1.198 & 1.416 \\
\hline Obs. & 9183 & & & & 13064 & & & & 22247 & & & \\
\hline \multicolumn{13}{|c|}{ Panel B: Wisconsin Longitudinal Study } \\
\hline Income PGI & & $\begin{array}{l}0.048 * * * \\
(0.012)\end{array}$ & & $\begin{array}{c}0.024 \\
(0.014)\end{array}$ & & $\begin{array}{c}0.068 * * * \\
(0.011)\end{array}$ & & $\begin{array}{c}0.048 * * * \\
(0.014)\end{array}$ & & $\begin{array}{c}0.058 * * * \\
(0.008)\end{array}$ & & $\begin{array}{c}0.036^{* * *} \\
(0.010)\end{array}$ \\
\hline EA PGI & & & $\begin{array}{l}0.060 * * * \\
(0.012)\end{array}$ & $\begin{array}{c}0.047 * * * \\
(0.014)\end{array}$ & & & $\begin{array}{c}0.063 * * * \\
(0.011)\end{array}$ & $\begin{array}{c}0.035 * * \\
(0.014)\end{array}$ & & & $\begin{array}{l}0.061 * * * \\
(0.008)\end{array}$ & $\begin{array}{c}0.041 * * * \\
(0.010)\end{array}$ \\
\hline$R^{2}(\%)$ & 2.082 & 2.560 & 2.785 & 2.873 & 1.915 & 2.859 & 2.704 & 3.035 & 19.172 & 19.743 & 19.787 & 19.943 \\
\hline$\Delta R^{2}(\%)$ & & 0.478 & 0.702 & 0.791 & & 0.944 & 0.789 & 1.120 & & 0.571 & 0.615 & 0.771 \\
\hline partial $R^{2}(\%)$ & & 0.488 & 0.717 & 0.807 & & 0.963 & 0.804 & 1.142 & & 0.706 & 0.761 & 0.954 \\
\hline Obs. & 3650 & & & & 3737 & & & & 7387 & & & \\
\hline
\end{tabular}

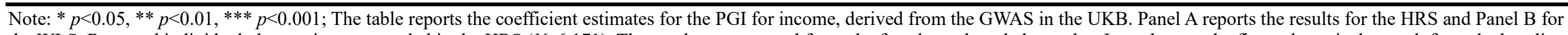

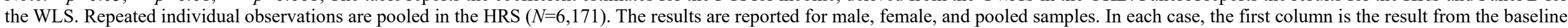
model. The baseline model is a regression of the log hourly wage on the covariates, which include age (with square and cube), dummy variables for male in the pooled analyses, year of observation, part-time worker (HRS only), genotyping batches (HRS only), the first 20 genetic principal components, as well as an interaction terms between the male dummy and the rest of covariates for the

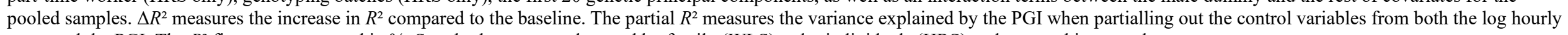
wage and the PGI. The $R^{2}$ figures are reported in \%. Standard errors are clustered by family (WLS) or by individuals (HRS) and reported in parentheses. 
Table A8. Associations between polygenic indices for income and 3-year moving averages of self-reported wages in the Health and Retirement Study

\begin{tabular}{|c|c|c|c|c|c|c|c|c|c|c|c|c|}
\hline \multirow[b]{2}{*}{ Income PGI } & \multicolumn{4}{|c|}{ Male } & \multicolumn{4}{|c|}{ Female } & \multicolumn{4}{|c|}{ Male+Female } \\
\hline & & $\begin{array}{c}0.078 * * * \\
(0.016)\end{array}$ & & $\begin{array}{c}0.046^{*} \\
(0.020)\end{array}$ & & $\begin{array}{c}0.071 * * * \\
(0.013)\end{array}$ & & $\begin{array}{l}0.035^{*} \\
(0.015)\end{array}$ & & $\begin{array}{c}0.074 * * * \\
(0.010)\end{array}$ & & $\begin{array}{c}0.039 * * \\
(0.012)\end{array}$ \\
\hline EA PGI & & & $\begin{array}{c}0.088 * * * \\
(0.015)\end{array}$ & $\begin{array}{c}0.061 * * \\
(0.020)\end{array}$ & & & $\begin{array}{c}0.087 * * * \\
(0.013)\end{array}$ & $\begin{array}{c}0.067 * * * \\
(0.016)\end{array}$ & & & $\begin{array}{c}0.087 * * * \\
(0.010)\end{array}$ & $\begin{array}{c}0.065^{* * *} \\
(0.012)\end{array}$ \\
\hline$R^{2}(\%)$ & 4.087 & 5.675 & 5.900 & 6.263 & 5.981 & 7.409 & 7.992 & 8.243 & 13.476 & 14.834 & 15.239 & 15.506 \\
\hline$\Delta R^{2}(\%)$ & & 1.588 & 1.813 & 2.176 & & 1.427 & 2.010 & 2.262 & & 1.358 & 1.763 & 2.030 \\
\hline partial $R^{2}(\%)$ & & 1.655 & 1.89 & 2.269 & & 1.518 & 2.138 & 2.406 & & 1.57 & 2.038 & 2.346 \\
\hline Obs. & 4,144 & & & & 6,768 & & & & 10,912 & & & \\
\hline
\end{tabular}

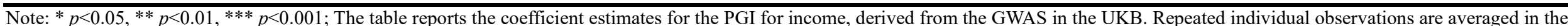

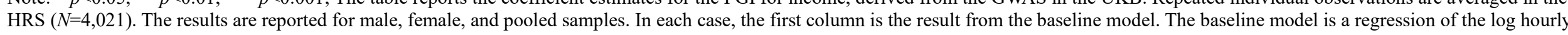
wage on the covariates, which include age (with square and cube), dummy variables for male in the pooled analyses, year of observation, part-time worker, and genotyping batches, the first 20 genetic principal components, as well as an interaction terms between the male dummy and the rest of covariates for the pooled samples. $\Delta R^{2}$ measures the increase in $R^{2}$ compared to the baseline. The partial $R^{2}$ measures the variance explained by the PGI when partialling out the control variables from both the log hourly wage and the PGI. The $R^{2}$ figures are reported in $\%$. Standard errors are clustered by individuals and reported in parentheses. 
Table A9. Comparison of predictive power of polygenic indices in UK Biobank sibling sample.

\begin{tabular}{lccc}
\hline & Income PGI $\Delta R^{2}$ & MTAG PGI $\Delta R^{2}$ & $N$ \\
\hline Log occupational wage per hour & $2.77 \%$ & $4.47 \%$ & 17,690 \\
Years of education & $4.18 \%$ & $7.26 \%$ & 35,128 \\
BMI & $0.66 \%$ & $1.40 \%$ & 35,428 \\
\hline
\end{tabular}

Note: The table reports the change in $R^{2}$ when a polygenic indice is added to the model. As covariates, all analyses include dummy variables for the year of birth, male, and being the younger sibling as well as the first 20 genetic PCs. For occupational wages, we use age dummies instead of the year of birth and add dummies for the year of survey. For BMI ratios we also control for the age dummies instead but not for the year of survey. In every case, we also include the interaction terms between the male dummy and the rest of covariates. 
Table A10. Estimates of 3-year moving-average income with respect to schooling and genetic factors in the Health and Retirement Study

\begin{tabular}{|c|c|c|c|c|c|c|c|c|c|c|c|c|c|c|c|c|}
\hline \multicolumn{2}{|c|}{ Panel A: Male + Female } & \multicolumn{3}{|c|}{ no PGI } & \multicolumn{4}{|c|}{ naive control } & \multicolumn{4}{|c|}{ GIV-C } & \multicolumn{4}{|c|}{ GIV-U } \\
\hline Educ & $\begin{array}{c}0.1107^{* * *} \\
(0.008)\end{array}$ & $\begin{array}{c}0.1055^{* * *} \\
(0.008)\end{array}$ & $\begin{array}{c}0.0920^{* * *} \\
(0.012)\end{array}$ & $\begin{array}{c}0.0857^{* * *} \\
(0.012)\end{array}$ & $\begin{array}{c}0.1076^{* * *} \\
(0.008)\end{array}$ & $\begin{array}{c}0.1026^{* * *} \\
(0.008)\end{array}$ & $\begin{array}{c}0.0900^{* * *} \\
(0.012)\end{array}$ & $\begin{array}{c}0.0840 * * * \\
(0.012)\end{array}$ & $\begin{array}{c}0.1048^{* * *} \\
(0.009)\end{array}$ & $\begin{array}{c}0.0997^{* * *} \\
(0.009)\end{array}$ & $\begin{array}{c}0.0890^{* * *} \\
(0.013)\end{array}$ & $\begin{array}{c}0.0826^{* * *} \\
(0.013)\end{array}$ & $\begin{array}{c}0.1040^{* * * *} \\
(0.009)\end{array}$ & $\begin{array}{c}0.0992^{* * *} \\
(0.009)\end{array}$ & $\begin{array}{c}0.0880^{* * *} \\
(0.012)\end{array}$ & $\begin{array}{c}0.0821 * * * \\
(0.013)\end{array}$ \\
\hline Educ $\times$ College & & & $\begin{array}{l}0.0615 \\
(0.034)\end{array}$ & $\begin{array}{l}0.0603 \\
(0.034)\end{array}$ & & & $\begin{array}{l}0.0594 \\
(0.034)\end{array}$ & $\begin{array}{l}0.0584 \\
(0.034)\end{array}$ & & & $\begin{array}{l}0.0582 \\
(0.035)\end{array}$ & $\begin{array}{l}0.0571 \\
(0.035)\end{array}$ & & & $\begin{array}{l}0.0548 \\
(0.034)\end{array}$ & $\begin{array}{l}0.0541 \\
(0.034)\end{array}$ \\
\hline Income PGI & & & & & $\begin{array}{c}0.0284 * * \\
(0.009)\end{array}$ & $\begin{array}{c}0.0272^{* *} \\
(0.009)\end{array}$ & $\begin{array}{c}0.0272^{* *} \\
(0.009)\end{array}$ & $\begin{array}{c}0.0258^{* *} \\
(0.009)\end{array}$ & $\begin{array}{c}0.1224 * \\
(0.056)\end{array}$ & $\begin{array}{l}0.1138^{*} \\
(0.055)\end{array}$ & $\begin{array}{l}0.1184 * \\
(0.056)\end{array}$ & $\begin{array}{l}0.1092 * \\
(0.054)\end{array}$ & $\begin{array}{l}0.0663 * \\
(0.030)\end{array}$ & $\begin{array}{c}0.0623 * \\
(0.029)\end{array}$ & $\begin{array}{c}0.0641^{*} \\
(0.029)\end{array}$ & $\begin{array}{c}0.0598^{*} \\
(0.029)\end{array}$ \\
\hline $\begin{array}{l}\text { Parental Educ } \\
\mathrm{R}^{2}\end{array}$ & 0.299 & $\begin{array}{c}\mathrm{Y} \\
0.301\end{array}$ & 0.301 & $\begin{array}{c}\mathrm{Y} \\
0.303\end{array}$ & 0.301 & $\begin{array}{c}\mathrm{Y} \\
0.303\end{array}$ & 0.303 & $\begin{array}{c}\mathrm{Y} \\
0.305\end{array}$ & & $\mathrm{Y}$ & & $\mathrm{Y}$ & & $\mathrm{Y}$ & & Y \\
\hline Obs. & 9906 & - & - & - & - & - & - & - & - & - & - & - & - & - & - & - \\
\hline Panel B: Male & & & & & & & & & & & & & & & & \\
\hline Educ & $\begin{array}{c}0.0880^{* * *} \\
(0.013)\end{array}$ & $\begin{array}{c}0.0813^{* * *} \\
(0.013)\end{array}$ & $\begin{array}{c}0.0437 * * \\
(0.016)\end{array}$ & $\begin{array}{c}0.0378^{*} \\
(0.016)\end{array}$ & $\begin{array}{c}0.0859 * * * \\
(0.013)\end{array}$ & $\begin{array}{c}0.0795^{* * *} \\
(0.013)\end{array}$ & $\begin{array}{c}0.0434 * * \\
(0.016)\end{array}$ & $\begin{array}{c}0.0376^{*} \\
(0.016)\end{array}$ & $\begin{array}{c}0.0821 * * * \\
(0.014)\end{array}$ & $\begin{array}{c}0.0763^{* * *} \\
(0.014)\end{array}$ & $\begin{array}{c}0.0483 * * \\
(0.016)\end{array}$ & $\begin{array}{c}0.0427^{* *} \\
(0.016)\end{array}$ & $\begin{array}{c}0.0805 * * * \\
(0.013)\end{array}$ & $\begin{array}{c}0.0743 * * * \\
(0.014)\end{array}$ & $\begin{array}{c}0.0437 * * \\
(0.016)\end{array}$ & $\begin{array}{l}0.0380^{*} \\
(0.016)\end{array}$ \\
\hline Educ $\times$ College & & & $\begin{array}{l}0.0353 \\
(0.050)\end{array}$ & $\begin{array}{l}0.0343 \\
(0.050)\end{array}$ & & & $\begin{array}{l}0.0337 \\
(0.050)\end{array}$ & $\begin{array}{l}0.0329 \\
(0.050)\end{array}$ & & & $\begin{array}{l}0.0400 \\
(0.053)\end{array}$ & $\begin{array}{l}0.0390 \\
(0.053)\end{array}$ & & & $\begin{array}{l}0.0247 \\
(0.051)\end{array}$ & $\begin{array}{l}0.0240 \\
(0.051)\end{array}$ \\
\hline Income PGI & & & & & $\begin{array}{l}0.0261 \\
(0.016)\end{array}$ & $\begin{array}{l}0.0246 \\
(0.015)\end{array}$ & $\begin{array}{l}0.0208 \\
(0.015)\end{array}$ & $\begin{array}{l}0.0194 \\
(0.015)\end{array}$ & $\begin{array}{l}0.1986^{*} \\
(0.100)\end{array}$ & $\begin{array}{l}0.1934 \\
(0.100)\end{array}$ & $\begin{array}{l}0.1814 \\
(0.102)\end{array}$ & $\begin{array}{l}0.1758 \\
(0.101)\end{array}$ & $\begin{array}{c}0.1057^{*} \\
(0.051)\end{array}$ & $\begin{array}{c}0.1035^{*} \\
(0.051)\end{array}$ & $\begin{array}{l}0.0954 \\
(0.052)\end{array}$ & $\begin{array}{l}0.0930 \\
(0.052)\end{array}$ \\
\hline Parental Educ & & Y & & Y & & Y & & Y & & $\mathrm{Y}$ & & $\mathrm{Y}$ & & $\mathrm{Y}$ & & Y \\
\hline $\mathrm{R}^{2}$ & 0.194 & 0.198 & 0.203 & 0.207 & 0.196 & 0.199 & 0.204 & 0.208 & & & & & & & & \\
\hline Obs. & 3784 & - & - & - & - & - & - & - & - & - & - & - & - & - & - & - \\
\hline Panel C: Female & & & & & & & & & & & & & & & & \\
\hline Educ & $\begin{array}{c}0.1248^{* * *} \\
(0.011)\end{array}$ & $\begin{array}{l}0.1205^{* * *} \\
(0.011)\end{array}$ & $\begin{array}{c}0.1208^{* * *} \\
(0.017)\end{array}$ & $\begin{array}{c}0.1152^{* * * *} \\
(0.018)\end{array}$ & $\begin{array}{c}0.1211^{* * *} \\
(0.011)\end{array}$ & $\begin{array}{c}0.1171^{* * *} \\
(0.011)\end{array}$ & $\begin{array}{c}0.1176^{* * *} \\
(0.017)\end{array}$ & $\begin{array}{c}0.1124 * * * \\
(0.018)\end{array}$ & $\begin{array}{c}0.1201 * * * \\
(0.012)\end{array}$ & $\begin{array}{c}0.1160^{* * *} \\
(0.012)\end{array}$ & $\begin{array}{c}0.1166^{* * * *} \\
(0.019)\end{array}$ & $\begin{array}{c}0.1110^{* * *} \\
(0.019)\end{array}$ & $\begin{array}{c}0.1197^{* * *} \\
(0.012)\end{array}$ & $\begin{array}{c}0.1159^{* * *} \\
(0.012)\end{array}$ & $\begin{array}{c}0.1167^{* * *} \\
(0.018)\end{array}$ & $\begin{array}{c}0.1115^{* * *} \\
(0.019)\end{array}$ \\
\hline Educ $\times$ College & & & $\begin{array}{l}0.0911 \\
(0.046)\end{array}$ & $\begin{array}{l}0.0893 \\
(0.046)\end{array}$ & & & $\begin{array}{l}0.0887 \\
(0.046)\end{array}$ & $\begin{array}{l}0.0871 \\
(0.046)\end{array}$ & & & $\begin{array}{l}0.0847 \\
(0.046)\end{array}$ & $\begin{array}{l}0.0835 \\
(0.046)\end{array}$ & & & $\begin{array}{l}0.0866 \\
(0.046)\end{array}$ & $\begin{array}{l}0.0854 \\
(0.046)\end{array}$ \\
\hline Income PGI & & & & & $\begin{array}{l}0.0290^{*} \\
(0.012)\end{array}$ & $\begin{array}{l}0.0278^{*} \\
(0.012)\end{array}$ & $\begin{array}{l}0.0285^{*} \\
(0.012)\end{array}$ & $\begin{array}{c}0.0274 * \\
(0.012)\end{array}$ & $\begin{array}{l}0.0786 \\
(0.066)\end{array}$ & $\begin{array}{l}0.0691 \\
(0.065)\end{array}$ & $\begin{array}{l}0.0752 \\
(0.067)\end{array}$ & $\begin{array}{l}0.0659 \\
(0.065)\end{array}$ & $\begin{array}{l}0.0430 \\
(0.036)\end{array}$ & $\begin{array}{l}0.0383 \\
(0.035)\end{array}$ & $\begin{array}{l}0.0410 \\
(0.036)\end{array}$ & $\begin{array}{l}0.0364 \\
(0.035)\end{array}$ \\
\hline Parental Educ & & Y & & $\mathrm{Y}$ & & $\mathrm{Y}$ & & $\mathrm{Y}$ & & $\mathrm{Y}$ & & $\mathrm{Y}$ & & Y & & Y \\
\hline $\mathrm{R}^{2}$ & 0.249 & 0.251 & 0.251 & 0.253 & 0.252 & 0.254 & 0.253 & 0.255 & & & & & & & & \\
\hline Obs. & 6122 & - & - & - & - & - & - & - & - & - & - & - & - & - & - & - \\
\hline
\end{tabular}


Table A11. Average wages of major occupation groups in the UK (listed in order of highest wages)

\begin{tabular}{lc}
\hline \multicolumn{1}{c}{ Major occupation group } & $\begin{array}{c}\text { Weekly wage averaged } \\
\text { over 2006-2010 (£) }\end{array}$ \\
\hline 1. Managers and Senior Officials & 807.74 \\
2. Professional Occupations & 688.72 \\
3. Associate Professional and Technical Occupations & 526.3 \\
5. Skilled Trades Occupations & 451.32 \\
8. Process, Plant and Machine Operatives & 412.68 \\
4. Administrative and Secretarial Occupations & 322.38 \\
6. Personal Service Occupations & 246.9 \\
9. Elementary Occupations & 232.38 \\
7. Sales and Customer Service Occupations & 202.32 \\
\hline
\end{tabular}

Source: The Annual Survey of Hours and Earnings 


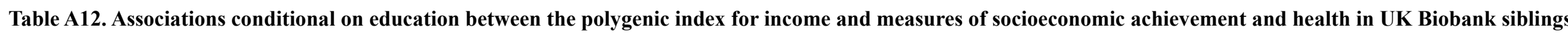

\begin{tabular}{|c|c|c|c|c|}
\hline & \multicolumn{4}{|c|}{ Conditional on education } \\
\hline & OLS & OLS-FE & GIV & GIV-FE \\
\hline \multicolumn{5}{|l|}{ Socioeconomic outcomes } \\
\hline log hourly wage & $0.019 * * *$ & $0.024 * *$ & $0.042 * * *$ & 0.042 \\
\hline$(\mathrm{N}=17,578)$ & $(0.002)$ & $(0.006)$ & $(0.008)$ & $(0.021)$ \\
\hline top household income & $0.018 * * *$ & 0.020 & $0.049 * * *$ & 0.064 \\
\hline$(\mathrm{N}=27,296)$ & $(0.003)$ & $(0.007)$ & $(0.009)$ & $(0.025)$ \\
\hline log regional income & $0.019 * * *$ & $0.011^{*}$ & $0.038 * * *$ & 0.033 \\
\hline$(\mathrm{N}=31,266)$ & $(0.002)$ & $(0.003)$ & $(0.005)$ & $(0.012)$ \\
\hline neighborhood score & $0.506^{* * *}$ & 0.428 & 0.850 & 1.212 \\
\hline$(\mathrm{N}=28,778)$ & $(0.091)$ & $(0.206)$ & $(0.308)$ & $(0.714)$ \\
\hline \multicolumn{5}{|l|}{ health proxies } \\
\hline waist-to-hip ratio & $-0.004 * * *$ & $-0.003 *$ & $-0.009 * * *$ & -0.007 \\
\hline$(\mathrm{N}=35,028)$ & $(0.000)$ & $(0.001)$ & $(0.001)$ & $(0.003)$ \\
\hline BMI & $-0.351 * * *$ & $-0.235^{* *}$ & $-0.576 * * *$ & -0.379 \\
\hline$(\mathrm{N}=34,968)$ & $(0.028)$ & $(0.064)$ & $(0.096)$ & $(0.230)$ \\
\hline blood pressure & $-0.612 * * *$ & -0.550 & $-1.254 * * *$ & -0.608 \\
\hline$(\mathrm{N}=31,372)$ & $(0.083)$ & $(0.212)$ & $(0.284)$ & $(0.758)$ \\
\hline lung function & $0.018 * *$ & 0.007 & 0.042 & 0.031 \\
\hline$(\mathrm{N}=29,844)$ & $(0.005)$ & $(0.013)$ & $(0.017)$ & $(0.048)$ \\
\hline \multicolumn{5}{|l|}{ disease diagnoses } \\
\hline ever hospitalized & $-0.013 * * *$ & -0.010 & -0.020 & -0.027 \\
\hline$(\mathrm{N}=35,132)$ & $(0.002)$ & $(0.006)$ & $(0.007)$ & $(0.021)$ \\
\hline ever diagnosed with cancer & -0.001 & 0.000 & -0.001 & 0.007 \\
\hline$(\mathrm{N}=35,132)$ & $(0.002)$ & $(0.006)$ & $(0.007)$ & $(0.022)$ \\
\hline infectious and parasitic diseases & $-0.007 * * *$ & -0.005 & -0.015 & 0.006 \\
\hline$(\mathrm{N}=35,132)$ & $(0.002)$ & $(0.005)$ & $(0.006)$ & $(0.017)$ \\
\hline neoplasms & -0.001 & 0.000 & -0.001 & 0.008 \\
\hline$(\mathrm{N}=35,132)$ & $(0.002)$ & $(0.006)$ & $(0.007)$ & $(0.021)$ \\
\hline diseases of blood organs and immune system & -0.006 & 0.002 & -0.012 & 0.005 \\
\hline$(\mathrm{N}=35,132)$ & $(0.002)$ & $(0.007)$ & $(0.008)$ & $(0.024)$ \\
\hline endocrine, nutritional, and metabolic diseases & $-0.013 * * *$ & -0.008 & -0.003 & 0.012 \\
\hline
\end{tabular}




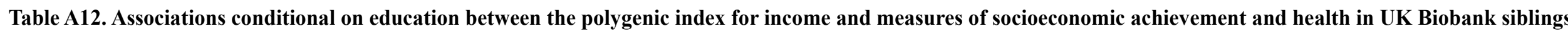

\begin{tabular}{|c|c|c|c|c|}
\hline & \multicolumn{4}{|c|}{ Conditional on education } \\
\hline & OLS & OLS-FE & GIV & GIV-FE \\
\hline$(\mathrm{N}=35,132)$ & $(0.002)$ & $(0.006)$ & $(0.008)$ & $(0.023)$ \\
\hline mental, behavioral, nervous system disorders & $-0.017 * * *$ & -0.006 & $-0.029 * *$ & 0.007 \\
\hline$(\mathrm{N}=35,132)$ & $(0.002)$ & $(0.006)$ & $(0.008)$ & $(0.022)$ \\
\hline diseases of the eye and adnexa & -0.003 & -0.004 & -0.011 & -0.015 \\
\hline$(\mathrm{N}=35,132)$ & $(0.002)$ & $(0.005)$ & $(0.006)$ & $(0.018)$ \\
\hline diseases of the circulatory system & $-0.021 * * *$ & -0.011 & $-0.039 * * *$ & -0.030 \\
\hline$(\mathrm{N}=35,132)$ & $(0.003)$ & $(0.007)$ & $(0.009)$ & $(0.026)$ \\
\hline diseases of the respiratory system & $-0.014 * * *$ & -0.008 & $-0.031 * * *$ & -0.021 \\
\hline$(\mathrm{N}=35,132)$ & $(0.002)$ & $(0.006)$ & $(0.007)$ & $(0.021)$ \\
\hline diseases of the digestive system & $-0.020 * * *$ & -0.010 & $-0.042 * * *$ & -0.038 \\
\hline$(\mathrm{N}=35,132)$ & $(0.003)$ & $(0.008)$ & $(0.010)$ & $(0.027)$ \\
\hline diseases of the skin and subcutaneous tissue & -0.005 & -0.003 & -0.005 & -0.011 \\
\hline$(\mathrm{N}=35,132)$ & $(0.002)$ & $(0.005)$ & $(0.006)$ & $(0.018)$ \\
\hline diseases of musculoskeletal system and connective tissue & $-0.024 * * *$ & -0.020 & $-0.042 * * *$ & -0.030 \\
\hline$(\mathrm{N}=35,132)$ & $(0.003)$ & $(0.007)$ & $(0.009)$ & $(0.026)$ \\
\hline diseases of genitourinary system & $-0.013 * * *$ & -0.010 & $-0.033 * *$ & -0.001 \\
\hline$(\mathrm{N}=35,132)$ & $(0.003)$ & $(0.007)$ & $(0.009)$ & $(0.025)$ \\
\hline symptoms and signs not elsewhere classified & $-0.021 * * *$ & -0.015 & $-0.041 * * *$ & -0.030 \\
\hline$(\mathrm{N}=35,132)$ & $(0.003)$ & $(0.008)$ & $(0.010)$ & $(0.028)$ \\
\hline injury, poisoning, and other consequences of external causes & -0.005 & -0.003 & -0.009 & -0.025 \\
\hline$(\mathrm{N}=35,132)$ & $(0.002)$ & $(0.006)$ & $(0.007)$ & $(0.022)$ \\
\hline external causes of morbidity and mortality & -0.006 & -0.003 & -0.011 & -0.029 \\
\hline$(\mathrm{N}=35,132)$ & $(0.002)$ & $(0.006)$ & $(0.008)$ & $(0.022)$ \\
\hline other health conditions & $-0.021 * * *$ & -0.020 & $-0.033 * *$ & -0.038 \\
\hline$(\mathrm{N}=35,132)$ & $(0.003)$ & $(0.008)$ & $(0.010)$ & $(0.028)$ \\
\hline
\end{tabular}

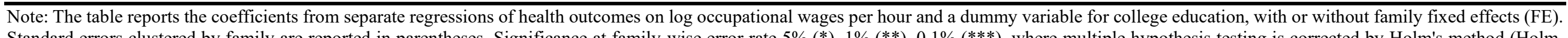

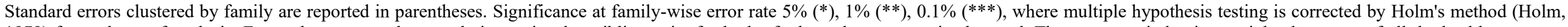

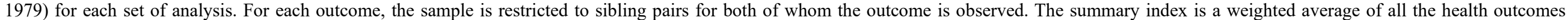

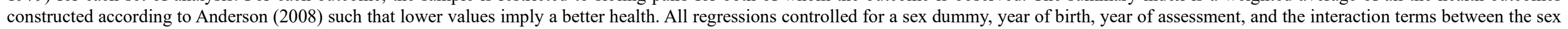
dummy and all other covariates. Regressions on log hourly wages also included dummies for year and age of observation. 
Table A13. Associations of polygenic index for income (without MTAG) in UK Biobank sibling pairs

\begin{tabular}{|c|c|c|c|c|}
\hline & \multirow[b]{2}{*}{ OLS } & \multirow[b]{2}{*}{ OLS-FE } & \multicolumn{2}{|c|}{ Conditional on education } \\
\hline & & & OLS & OLS-FE \\
\hline \multicolumn{5}{|l|}{ Socioeconomic outcomes } \\
\hline log hourly wage & $0.0581^{* * *}$ & $0.0325^{* * *}$ & $0.0158^{* * *}$ & 0.0161 \\
\hline$(N=17,692 \mid 17,578)$ & $(0.003)$ & $(0.006)$ & $(0.002)$ & $(0.006)$ \\
\hline top household income & $0.0459 * * *$ & $0.0280 * *$ & $0.0167 * * *$ & 0.0181 \\
\hline$(N=27,412 \mid 27,296)$ & $(0.003)$ & $(0.007)$ & $(0.003)$ & $(0.007)$ \\
\hline log regional income & $0.0315^{* * *}$ & $0.0143 * * *$ & $0.0143 * * *$ & $0.0112^{*}$ \\
\hline$(N=31,692 \mid 31,266)$ & $(0.001)$ & $(0.003)$ & $(0.001)$ & $(0.003)$ \\
\hline neighborhood score & $1.1489^{* * *}$ & $0.7167 * *$ & $0.3561 * * *$ & 0.5556 \\
\hline$(N=29,166 \mid 28,778)$ & $(0.088)$ & $(0.196)$ & $(0.088)$ & $(0.198)$ \\
\hline years of education & $1.0508^{* * *}$ & $0.5683 * * *$ & & \\
\hline$(N=35,132 \mid \mathrm{NA})$ & $(0.027)$ & $(0.065)$ & & \\
\hline college degree & $0.0986^{* * *}$ & $0.0500 * * *$ & & \\
\hline$(N=35,132 \mid \mathrm{NA})$ & $(0.002)$ & $(0.006)$ & & \\
\hline \multicolumn{5}{|l|}{ health proxies } \\
\hline waist-to-hip ratio & $-0.0051 * * *$ & -0.0029 & $-0.0028 * * *$ & -0.0022 \\
\hline$(N=35,498 \mid 35,028)$ & $(0.000)$ & $(0.001)$ & $(0.000)$ & $(0.001)$ \\
\hline BMI & $-0.3844 * * *$ & -0.1559 & $-0.2051 * * *$ & -0.1132 \\
\hline$(N=35,432 \mid 34,968)$ & $(0.027)$ & $(0.062)$ & $(0.027)$ & $(0.062)$ \\
\hline blood pressure & $-0.6487 * * *$ & -0.2545 & $-0.4445 * * *$ & -0.1968 \\
\hline$(N=31,770 \mid 31,372)$ & $(0.078)$ & $(0.200)$ & $(0.081)$ & $(0.203)$ \\
\hline lung function & $0.0453 * * *$ & 0.0044 & $0.0168 * *$ & -0.0032 \\
\hline$(N=30,240 \mid 29,844)$ & $(0.005)$ & $(0.012)$ & $(0.005)$ & $(0.012)$ \\
\hline \multicolumn{5}{|l|}{ disease diagnoses } \\
\hline ever hospitalized & $-0.0141 * * *$ & -0.0066 & $-0.0072 * *$ & -0.0053 \\
\hline$(N=35,602 \mid 35,132)$ & $(0.002)$ & $(0.006)$ & $(0.002)$ & $(0.006)$ \\
\hline ever diagnosed with cancer & 0.0001 & 0.0028 & -0.0002 & 0.0017 \\
\hline$(N=35,602 \mid 35,132)$ & $(0.002)$ & $(0.006)$ & $(0.002)$ & $(0.006)$ \\
\hline infectious and parasitic diseases & $-0.0088 * * *$ & 0.0007 & -0.0045 & 0.0012 \\
\hline$(N=35,602 \mid 35,132)$ & $(0.002)$ & $(0.005)$ & $(0.002)$ & $(0.005)$ \\
\hline neoplasms & 0.0004 & 0.0024 & -0.0004 & 0.0014 \\
\hline$(N=35,602 \mid 35,132)$ & $(0.002)$ & $(0.006)$ & $(0.002)$ & $(0.006)$ \\
\hline diseases of blood organs and immune system & $-0.0088 * * *$ & -0.0007 & -0.0039 & 0.0004 \\
\hline$(N=35,602 \mid 35,132)$ & $(0.002)$ & $(0.006)$ & $(0.002)$ & $(0.006)$ \\
\hline
\end{tabular}


Table A13. Associations of polygenic index for income (without MTAG) in UK Biobank sibling pairs

\begin{tabular}{|c|c|c|c|c|}
\hline & \multirow[b]{2}{*}{ OLS } & \multirow[b]{2}{*}{ OLS-FE } & \multicolumn{2}{|c|}{ Conditional on education } \\
\hline & & & OLS & OLS-FE \\
\hline endocrine, nutritional, and metabolic diseases & $-0.0152 * * *$ & -0.0032 & -0.0048 & -0.0008 \\
\hline$(N=35,602 \mid 35,132)$ & $(0.002)$ & $(0.006)$ & $(0.002)$ & $(0.006)$ \\
\hline mental, behavioral, nervous system disorders & $-0.0189 * * *$ & -0.0042 & $-0.0105 * * *$ & -0.0023 \\
\hline$(N=35,602 \mid 35,132)$ & $(0.002)$ & $(0.006)$ & $(0.002)$ & $(0.006)$ \\
\hline diseases of the eye and adnexa & $-0.0059 * *$ & -0.0031 & -0.0036 & -0.0022 \\
\hline$(N=35,602 \mid 35,132)$ & $(0.002)$ & $(0.005)$ & $(0.002)$ & $(0.005)$ \\
\hline diseases of the circulatory system & $-0.0264 * * *$ & -0.0128 & $-0.0149 * * *$ & -0.0105 \\
\hline$(N=35,602 \mid 35,132)$ & $(0.002)$ & $(0.007)$ & $(0.003)$ & $(0.007)$ \\
\hline diseases of the respiratory system & $-0.0173 * * *$ & -0.0075 & $-0.0109 * * *$ & -0.0057 \\
\hline$(N=35,602 \mid 35,132)$ & $(0.002)$ & $(0.006)$ & $(0.002)$ & $(0.006)$ \\
\hline diseases of the digestive system & $-0.0246 * * *$ & -0.0076 & $-0.0133 * * *$ & -0.0053 \\
\hline$(N=35,602 \mid 35,132)$ & $(0.003)$ & $(0.007)$ & $(0.003)$ & $(0.007)$ \\
\hline diseases of the skin and subcutaneous tissue & $-0.0047^{*}$ & -0.0014 & -0.0011 & 0.0000 \\
\hline$(N=35,602 \mid 35,132)$ & $(0.002)$ & $(0.005)$ & $(0.002)$ & $(0.005)$ \\
\hline diseases of musculoskeletal system and connective tissue & $-0.0237 * * *$ & -0.0096 & $-0.0136 * * *$ & -0.0070 \\
\hline$(N=35,602 \mid 35,132)$ & $(0.002)$ & $(0.007)$ & $(0.003)$ & $(0.007)$ \\
\hline diseases of genitourinary system & $-0.0162 * * *$ & -0.0036 & $-0.0080^{*}$ & -0.0017 \\
\hline$(N=35,602 \mid 35,132)$ & $(0.002)$ & $(0.007)$ & $(0.002)$ & $(0.007)$ \\
\hline symptoms and signs not elsewhere classified & $-0.0252 * * *$ & -0.0116 & $-0.0151 * * *$ & -0.0107 \\
\hline$(N=35,602 \mid 35,132)$ & $(0.003)$ & $(0.007)$ & $(0.003)$ & $(0.007)$ \\
\hline injury, poisoning, and other consequences of external causes & $-0.0073 * *$ & -0.0027 & -0.0042 & -0.0028 \\
\hline$(N=35,602 \mid 35,132)$ & $(0.002)$ & $(0.006)$ & $(0.002)$ & $(0.006)$ \\
\hline external causes of morbidity and mortality & $-0.0082 * * *$ & -0.0031 & -0.0044 & -0.0032 \\
\hline$(N=35,602 \mid 35,132)$ & $(0.002)$ & $(0.006)$ & $(0.002)$ & $(0.006)$ \\
\hline other health conditions & $-0.0222 * * *$ & -0.0145 & $-0.0126^{* * *}$ & -0.0129 \\
\hline$(N=35,602 \mid 35,132)$ & $(0.003)$ & $(0.008)$ & $(0.003)$ & $(0.008)$ \\
\hline
\end{tabular}

Note: Significance at family-wise error rate $5 \%(*), 1 \%(* *), 0.1 \%(* * *)$, where multiple hypothesis testing is corrected using Holm's method

(Holm, 1979) for each set of analysis. Standard errors clustered by family are reported in parentheses. The table reports the coefficient estimates for the standardized PGI for income (non-augmented). For each outcome, the sample is restricted to sibling pairs for both of whom the outcome is observed. FE indicates the models with family fixed effects included. The second column set reports the results where education is controlled for by including dummies for each qualification. As covariates we include dummy variables for the year of birth, male, and being a younger sibling as well as the top 20 genetic PCs. For the economic outcomes we control for the age dummies instead of the year of birth and add dummies for the year of survey. For BMI and waist-to-hip ratio we also control for the age dummies instead but not for the year of survey. In every case we also include the interaction terms between the male dummy and the rest of covariates. 
Table A14. Associations of polygenic index for educational attainment in UK Biobank sibling pairs

\begin{tabular}{|c|c|c|c|c|}
\hline & \multirow[b]{2}{*}{ OLS } & \multirow[b]{2}{*}{ OLS-FE } & \multicolumn{2}{|c|}{ Conditional on education } \\
\hline & & & OLS & OLS-FE \\
\hline \multicolumn{5}{|l|}{ Socioeconomic outcomes } \\
\hline log hourly wage & $0.0717^{* * *}$ & $0.0443^{* * *}$ & $0.0174 * * *$ & $0.0235^{* *}$ \\
\hline$(N=17,692 \mid 17,578)$ & $(0.002)$ & $(0.007)$ & $(0.002)$ & $(0.006)$ \\
\hline top household income & $0.0547 * * *$ & $0.0339 * * *$ & $0.0171 * * *$ & $0.0203 *$ \\
\hline$(N=27,412 \mid 27,296)$ & $(0.003)$ & $(0.007)$ & $(0.003)$ & $(0.007)$ \\
\hline log regional income & $0.0397 * * *$ & $0.0132 * *$ & $0.0185^{* * *}$ & $0.0093 *$ \\
\hline$(N=31,692 \mid 31,266)$ & $(0.001)$ & $(0.003)$ & $(0.002)$ & $(0.003)$ \\
\hline neighborhood score & $1.5003 * * *$ & 0.5229 & $0.5085^{* * *}$ & 0.3163 \\
\hline$(N=29,166 \mid 28,778)$ & $(0.088)$ & $(0.200)$ & $(0.091)$ & $(0.203)$ \\
\hline years of education & $1.3486^{* * *}$ & $0.7235^{* * *}$ & & \\
\hline$(N=35,132 \mid \mathrm{NA})$ & $(0.026)$ & $(0.066)$ & & \\
\hline college degree & $0.1281 * * *$ & $0.0662 * * *$ & & \\
\hline$(N=35,132 \mid \mathrm{NA})$ & $(0.002)$ & $(0.006)$ & & \\
\hline \multicolumn{5}{|l|}{ health proxies } \\
\hline waist-to-hip ratio & $-0.0063 * * *$ & $-0.0038 * *$ & $-0.0033^{* * *}$ & $-0.0029 *$ \\
\hline$(N=35,498 \mid 35,028)$ & $(0.000)$ & $(0.001)$ & $(0.000)$ & $(0.001)$ \\
\hline BMI & $-0.5545 * * *$ & $-0.2903 * * *$ & $-0.3452 * * *$ & $-0.2425 * *$ \\
\hline$(N=35,432 \mid 34,968)$ & $(0.027)$ & $(0.063)$ & $(0.028)$ & $(0.064)$ \\
\hline blood pressure & $-0.8190 * * *$ & $-0.6570^{*}$ & $-0.5865^{* * *}$ & $-0.5989 *$ \\
\hline$(N=31,770 \mid 31,372)$ & $(0.078)$ & $(0.208)$ & $(0.083)$ & $(0.212)$ \\
\hline lung function & $0.0521^{* * *}$ & 0.0192 & $0.0156^{* *}$ & 0.0102 \\
\hline$(N=30,240 \mid 29,844)$ & $(0.005)$ & $(0.013)$ & $(0.005)$ & $(0.013)$ \\
\hline \multicolumn{5}{|l|}{ disease diagnoses } \\
\hline ever hospitalized & $-0.0206 * * *$ & -0.0112 & $-0.0129 * * *$ & -0.0102 \\
\hline$(N=35,602 \mid 35,132)$ & $(0.002)$ & $(0.006)$ & $(0.002)$ & $(0.006)$ \\
\hline ever diagnosed with cancer & -0.0007 & -0.0004 & $-0.0009^{* *}$ & -0.0011 \\
\hline$(N=35,602 \mid 35,132)$ & $(0.002)$ & $(0.006)$ & $(0.002)$ & $(0.006)$ \\
\hline infectious and parasitic diseases & $-0.0125 * * *$ & -0.0067 & $-0.0068 * * *$ & -0.0057 \\
\hline$(N=35,602 \mid 35,132)$ & $(0.002)$ & $(0.005)$ & $(0.002)$ & $(0.005)$ \\
\hline neoplasms & 0.0006 & -0.0001 & -0.0004 & -0.0006 \\
\hline$(N=35,602 \mid 35,132)$ & $(0.002)$ & $(0.006)$ & $(0.002)$ & $(0.006)$ \\
\hline diseases of blood organs and immune system & $-0.0118 * * *$ & 0.0001 & $-0.0062 * *$ & 0.0008 \\
\hline$(N=35,602 \mid 35,132)$ & $(0.002)$ & $(0.007)$ & $(0.002)$ & $(0.007)$ \\
\hline
\end{tabular}


Table A14. Associations of polygenic index for educational attainment in UK Biobank sibling pairs

\begin{tabular}{|c|c|c|c|c|}
\hline & \multirow[b]{2}{*}{ OLS } & \multirow[b]{2}{*}{ OLS-FE } & \multicolumn{2}{|c|}{ Conditional on education } \\
\hline & & & OLS & OLS-FE \\
\hline endocrine, nutritional, and metabolic diseases & $-0.0270 * * *$ & -0.0136 & $-0.0152 * * *$ & -0.0114 \\
\hline$(N=35,602 \mid 35,132)$ & $(0.002)$ & $(0.006)$ & $(0.002)$ & $(0.006)$ \\
\hline mental, behavioral, nervous system disorders & $-0.0275 * * *$ & -0.0084 & $-0.0174 * * *$ & -0.0055 \\
\hline$(N=35,602 \mid 35,132)$ & $(0.002)$ & $(0.006)$ & $(0.002)$ & $(0.006)$ \\
\hline diseases of the eye and adnexa & $-0.0060^{* *}$ & -0.0062 & $-0.0031 * *$ & -0.0053 \\
\hline$(N=35,602 \mid 35,132)$ & $(0.002)$ & $(0.005)$ & $(0.002)$ & $(0.005)$ \\
\hline diseases of the circulatory system & $-0.0348 * * *$ & -0.0119 & $-0.0211 * * *$ & -0.0098 \\
\hline$(N=35,602 \mid 35,132)$ & $(0.003)$ & $(0.007)$ & $(0.003)$ & $(0.007)$ \\
\hline diseases of the respiratory system & $-0.0209 * * *$ & -0.0079 & $-0.0131 * * *$ & -0.0062 \\
\hline$(N=35,602 \mid 35,132)$ & $(0.002)$ & $(0.006)$ & $(0.002)$ & $(0.006)$ \\
\hline diseases of the digestive system & $-0.0335 * * *$ & -0.0143 & $-0.0202 * * *$ & -0.0118 \\
\hline$(N=35,602 \mid 35,132)$ & $(0.003)$ & $(0.008)$ & $(0.003)$ & $(0.008)$ \\
\hline diseases of the skin and subcutaneous tissue & $-0.0082 * * *$ & -0.0040 & $-0.0048^{* *}$ & -0.0024 \\
\hline$(N=35,602 \mid 35,132)$ & $(0.002)$ & $(0.005)$ & $(0.002)$ & $(0.005)$ \\
\hline diseases of musculoskeletal system and connective tissue & $-0.0354 * * *$ & $-0.0256 * *$ & $-0.0241 * * *$ & $-0.0229 *$ \\
\hline$(N=35,602 \mid 35,132)$ & $(0.002)$ & $(0.007)$ & $(0.003)$ & $(0.007)$ \\
\hline diseases of genitourinary system & $-0.0220 * * *$ & -0.0111 & $-0.0128 * * *$ & -0.0099 \\
\hline$(N=35,602 \mid 35,132)$ & $(0.002)$ & $(0.007)$ & $(0.003)$ & $(0.007)$ \\
\hline symptoms and signs not elsewhere classified & $-0.0317 * * *$ & -0.0136 & $-0.0200 * * *$ & -0.0127 \\
\hline$(N=35,602 \mid 35,132)$ & $(0.003)$ & $(0.008)$ & $(0.003)$ & $(0.008)$ \\
\hline injury, poisoning, and other consequences of external causes & $-0.0088 * * *$ & -0.0035 & $-0.0051^{* *}$ & -0.0023 \\
\hline$(N=35,602 \mid 35,132)$ & $(0.002)$ & $(0.006)$ & $(0.002)$ & $(0.006)$ \\
\hline external causes of morbidity and mortality & $-0.0106^{* * *}$ & -0.0038 & $-0.0061 * *$ & -0.0028 \\
\hline$(N=35,602 \mid 35,132)$ & $(0.002)$ & $(0.006)$ & $(0.002)$ & $(0.006)$ \\
\hline other health conditions & $-0.0325 * * *$ & $-0.0243^{*}$ & $-0.0219 * * *$ & $-0.0224 *$ \\
\hline$(N=35,602 \mid 35,132)$ & $(0.003)$ & $(0.008)$ & $(0.003)$ & $(0.008)$ \\
\hline
\end{tabular}

Note: Significance at family-wise error rate $5 \%(*), 1 \%(* *), 0.1 \%(* * *)$, where multiple hypothesis testing is corrected using Holm's method

(Holm, 1979) for each set of analysis. Standard errors clustered by family are reported in parentheses. The table reports the coefficient estimates for the standardized PGI for educational attainment. For each outcome, the sample is restricted to sibling pairs for both of whom the outcome is observed. FE indicates the models with family fixed effects included. The second column set reports the results where education is controlled for by including dummies for each qualification. As covariates we include dummy variables for the year of birth, male, and being a younger sibling as well as the top 20 genetic PCs. For the economic outcomes we control for the age dummies instead of the year of birth and add dummies for the year of survey. For BMI and waist-to-hip ratio we also control for the age dummies instead but not for the year of survey. In every case we also include the interaction terms between the male dummy and the rest of covariates. 


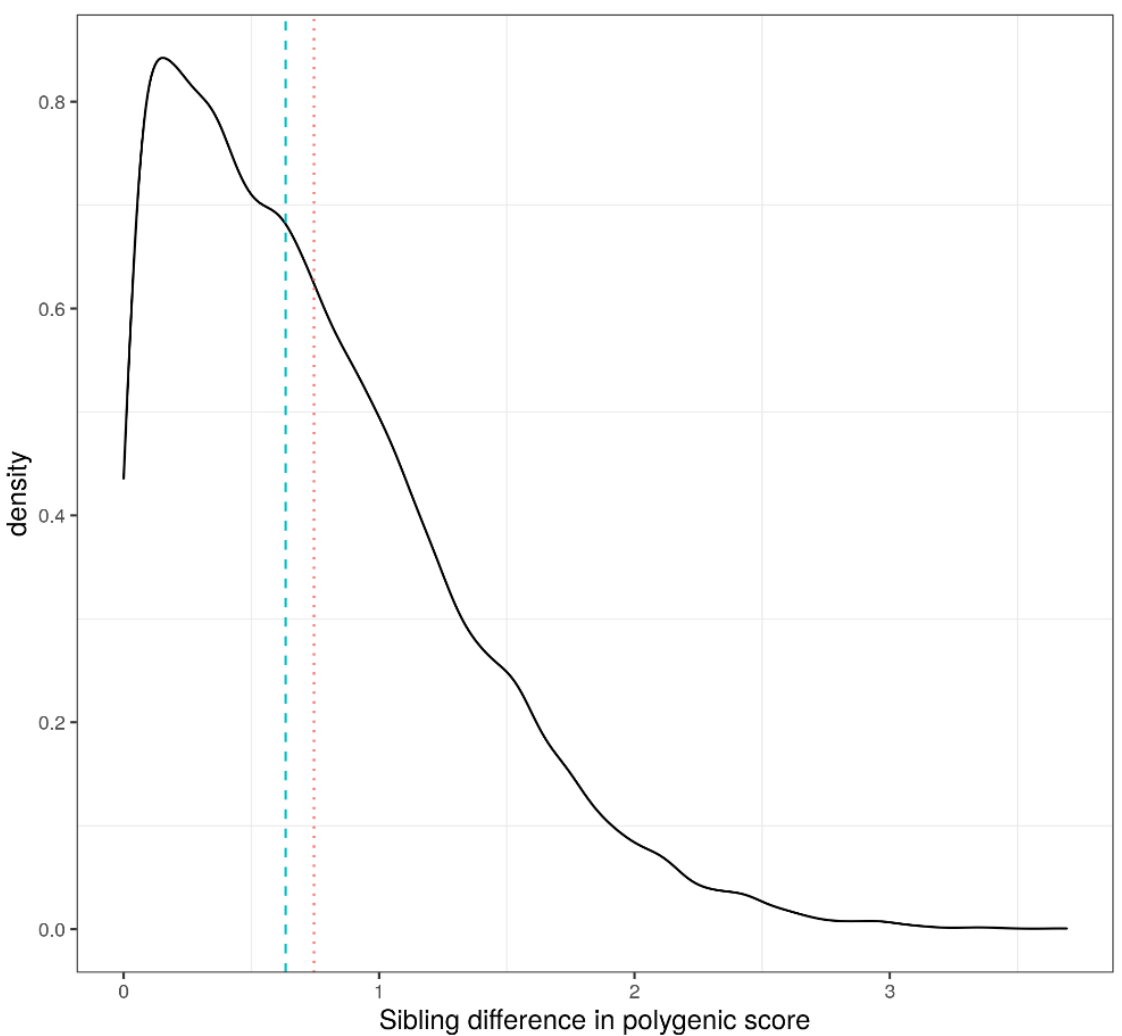

$\begin{array}{l:c}\text { mean }=0.745 & \text { median }=0.634\end{array}$

Figure A1. Distribution of the differences in MTAG polygenic indices (PGI) for income between siblings in the UK Biobank Note: The figure plots the density distribution of the absolute difference in the standardized PGI between siblings with Gaussian kernel. 


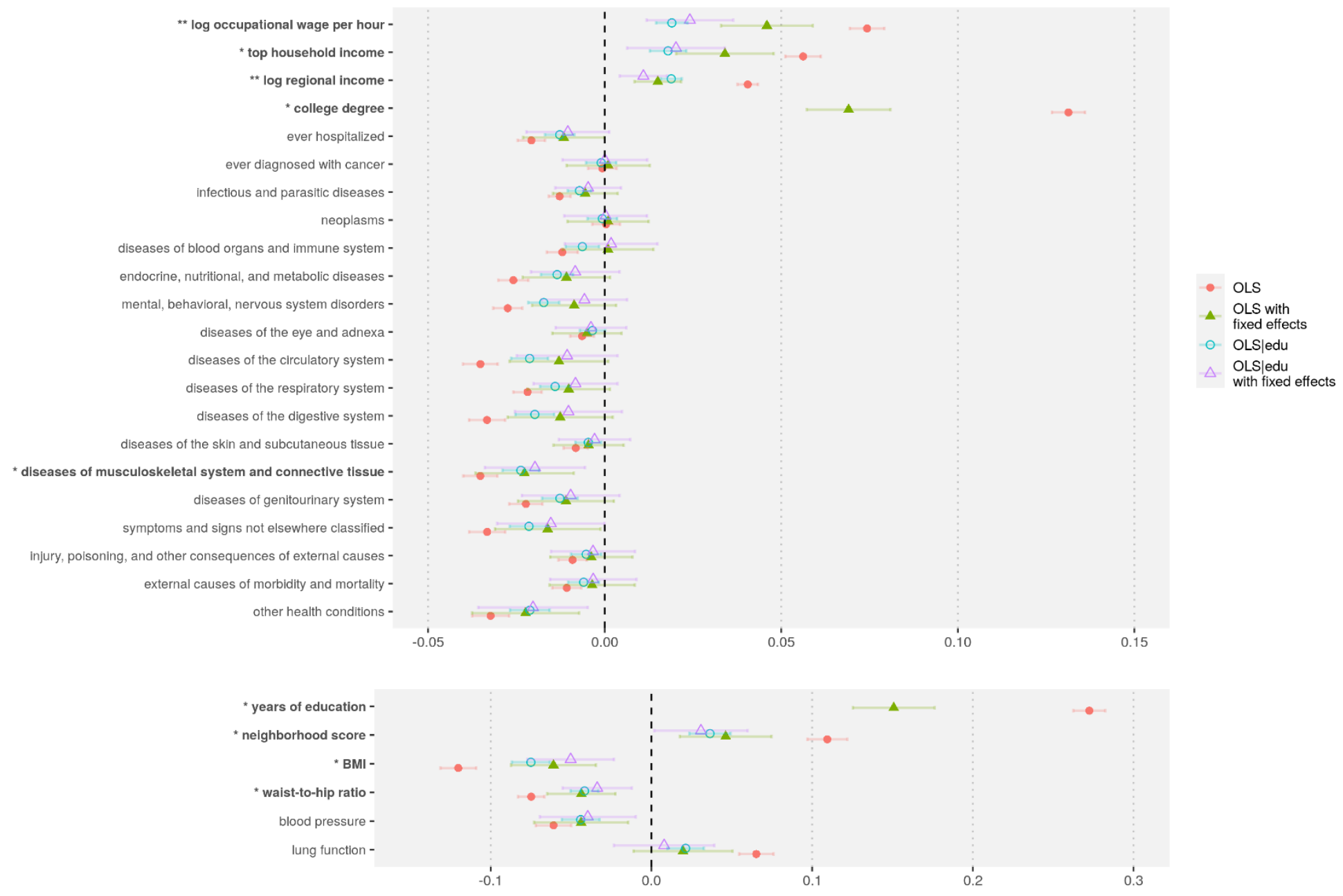

Figure A2. Associations of MTAG polygenic index (PGI) for income in UK Biobank sibling pairs (OLS)

Note: The figure plots the regression coefficients for the standardized MTAG income PGI estimated by OLS with or without education controlled for. Error bars are $95 \%$ confidence intervals. The upper panel shows the estimates measured on percentage scale. The lower panel plots the standardized estimates (i.e., the outcomes and the PGI are both standardized). One asterisk indicates significance at the $5 \%$ family-wise error rate for the estimate with family fixed effects while two asterisks indicate the significance conditional on education. Multiple testing is corrected for using Holm's method (Holm, 1979). See Table 5 and 6 for more details. 


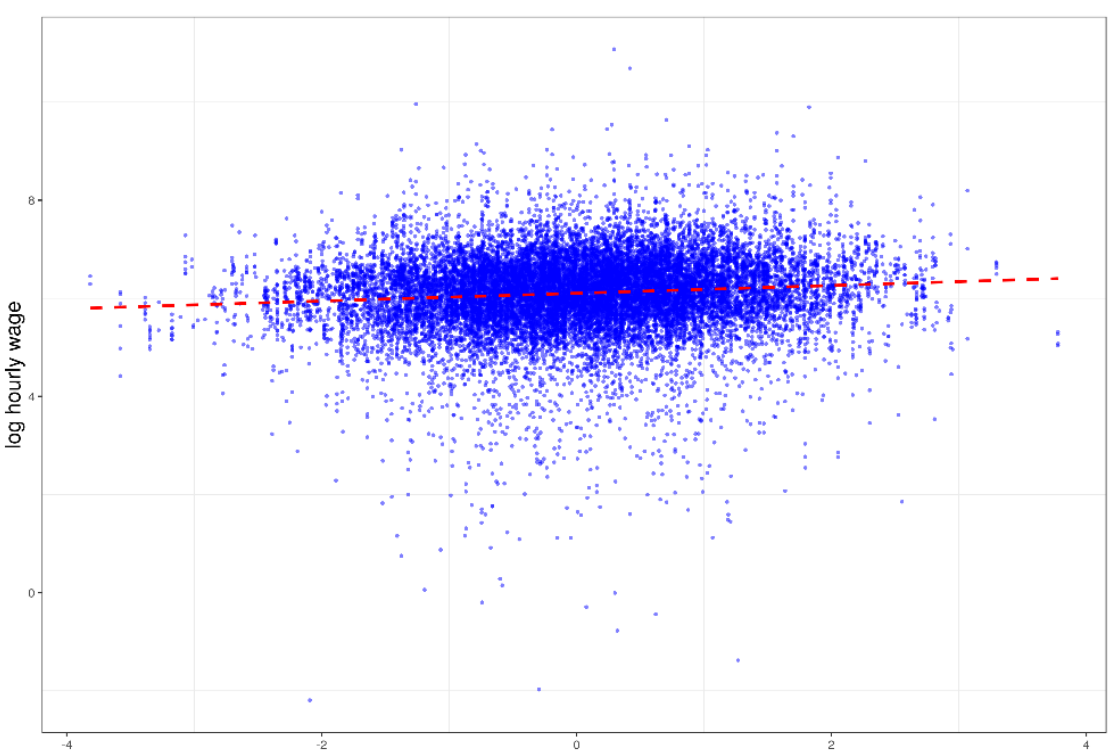

polygenic score

a. HRS

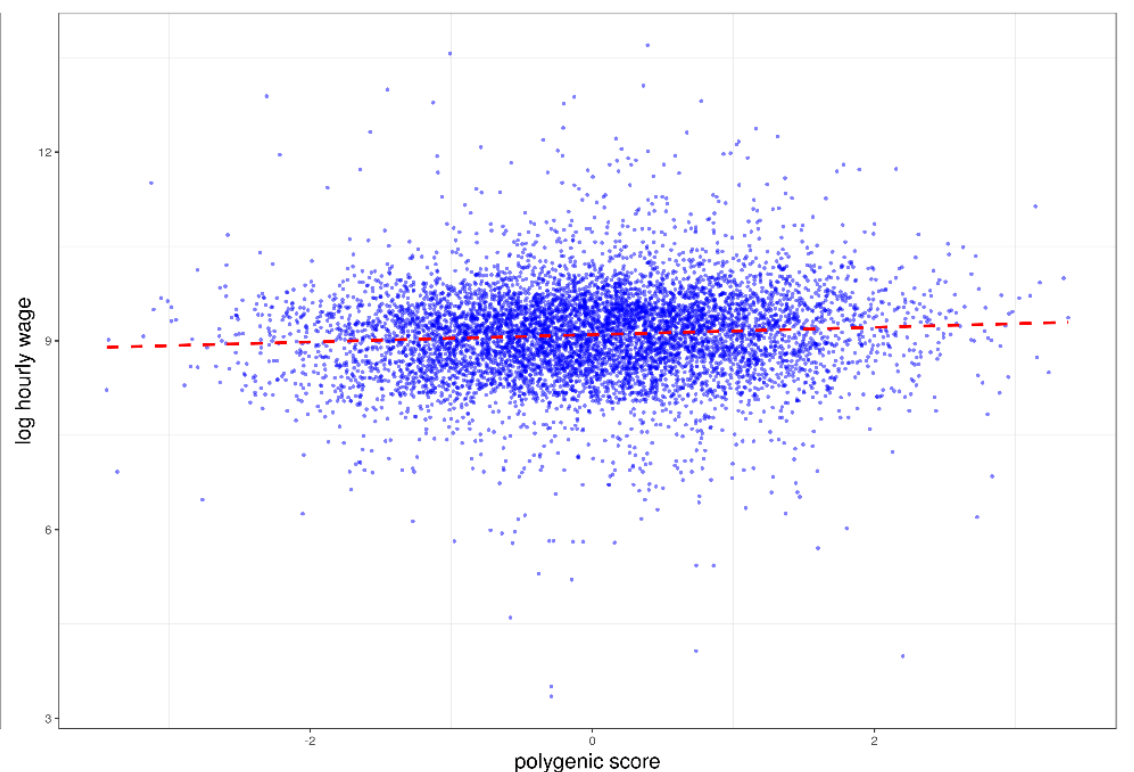

b. WLS

\section{Figure A3. Scatter plots of log hourly wages and polygenic indices for income}

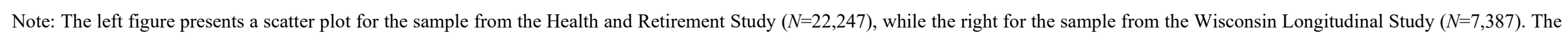
dotted lines are regression lines. 


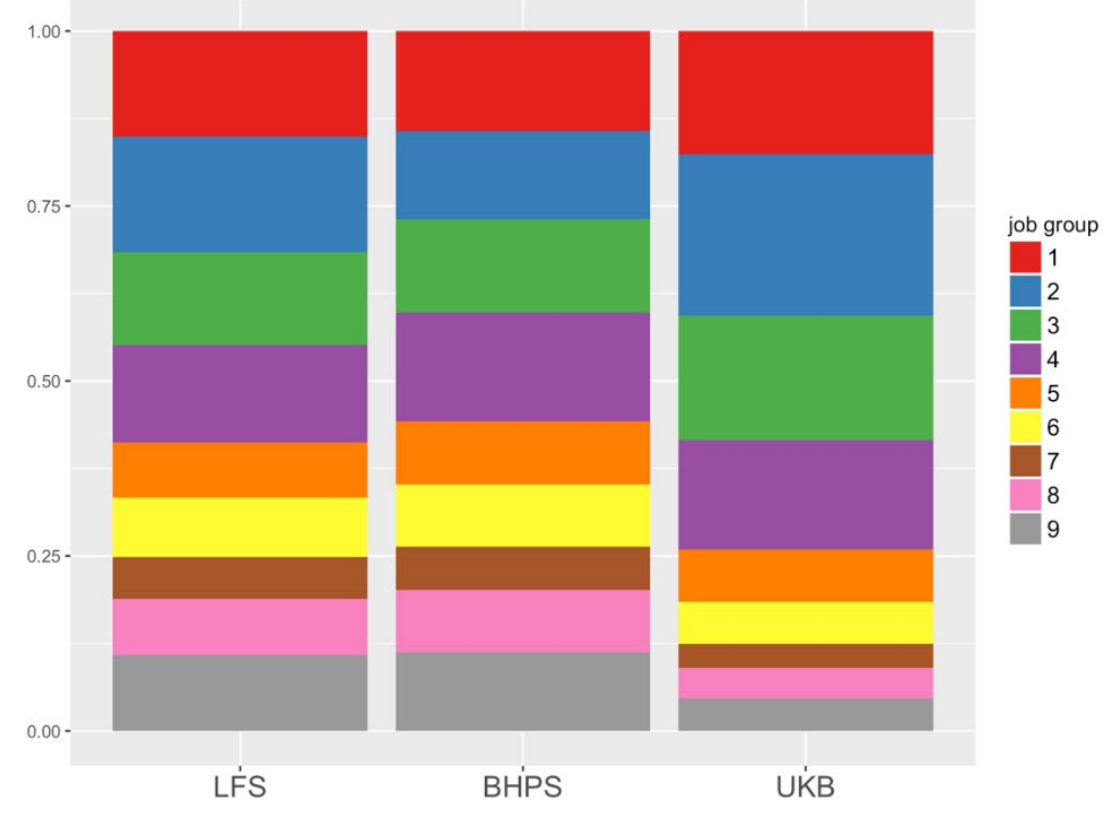

Figure A4. The composition of major occupation groups in the UK

Note: see Table A11 for the major occupation group reference. 

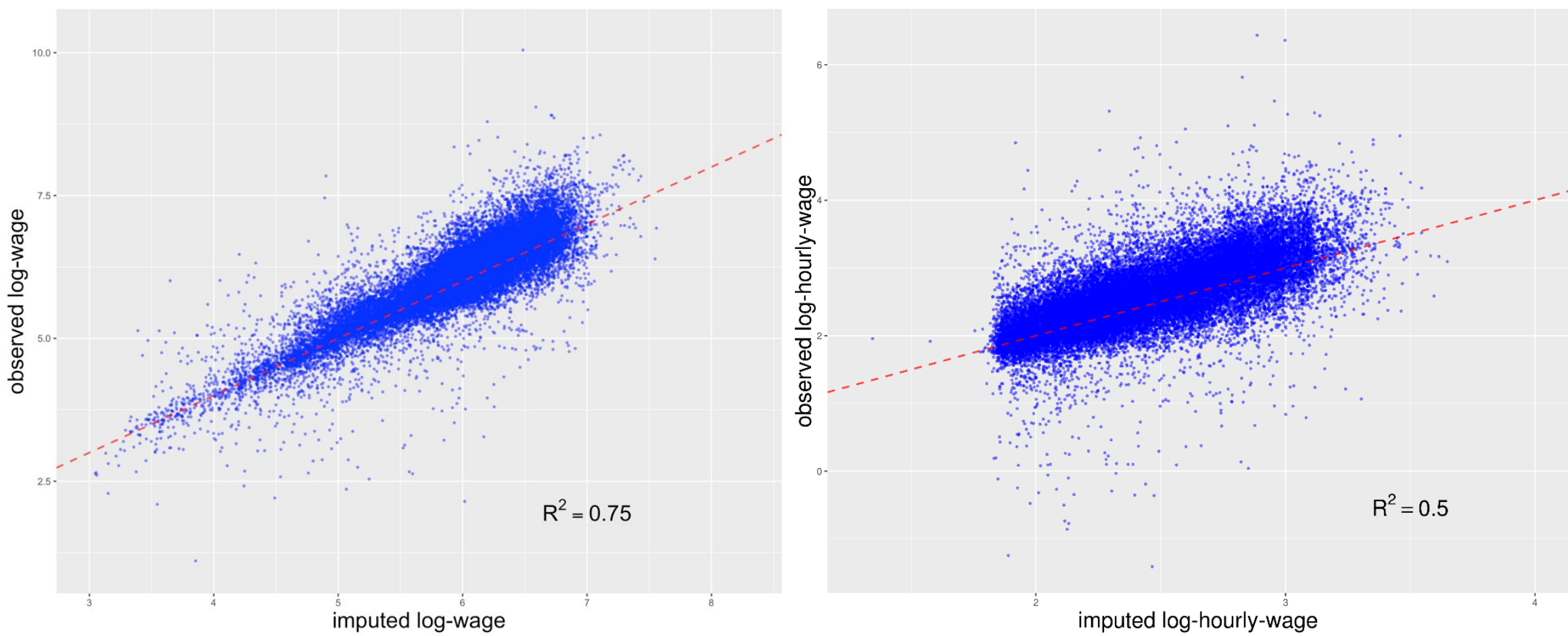

Figure A5. Scatter plots of imputed and observed log-wages in the BHPS $(N=32,947)$ 


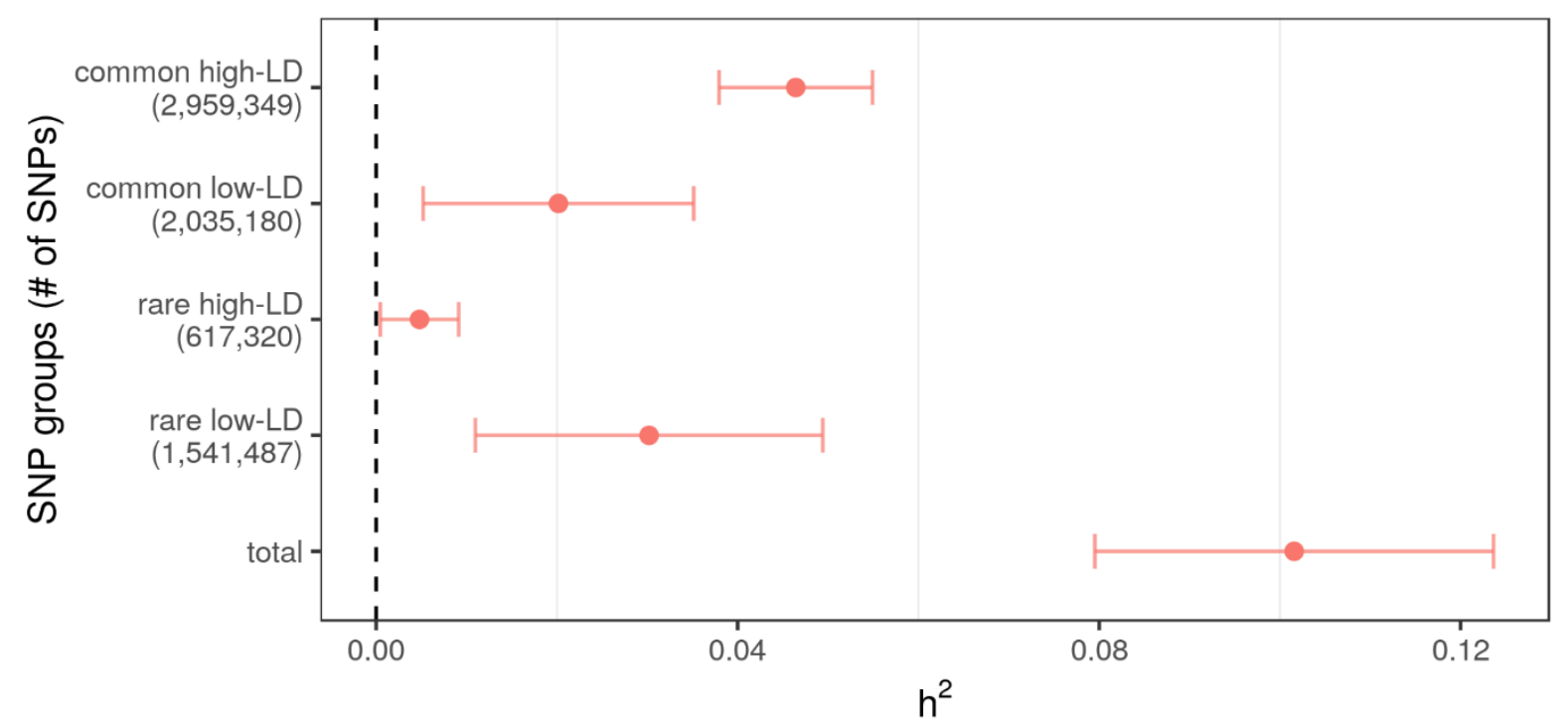

Figure A6. Decomposition of the total heritability of occupational wages by different groups of single nucleotide polymorphisms (SNPs)

Note: GREML estimation in 24,000 unrelated individuals from the UK Biobank. Error bars are standard errors. SNPs are sorted into four groups by different genetic features. LD - linkage disequilibrium scores. 


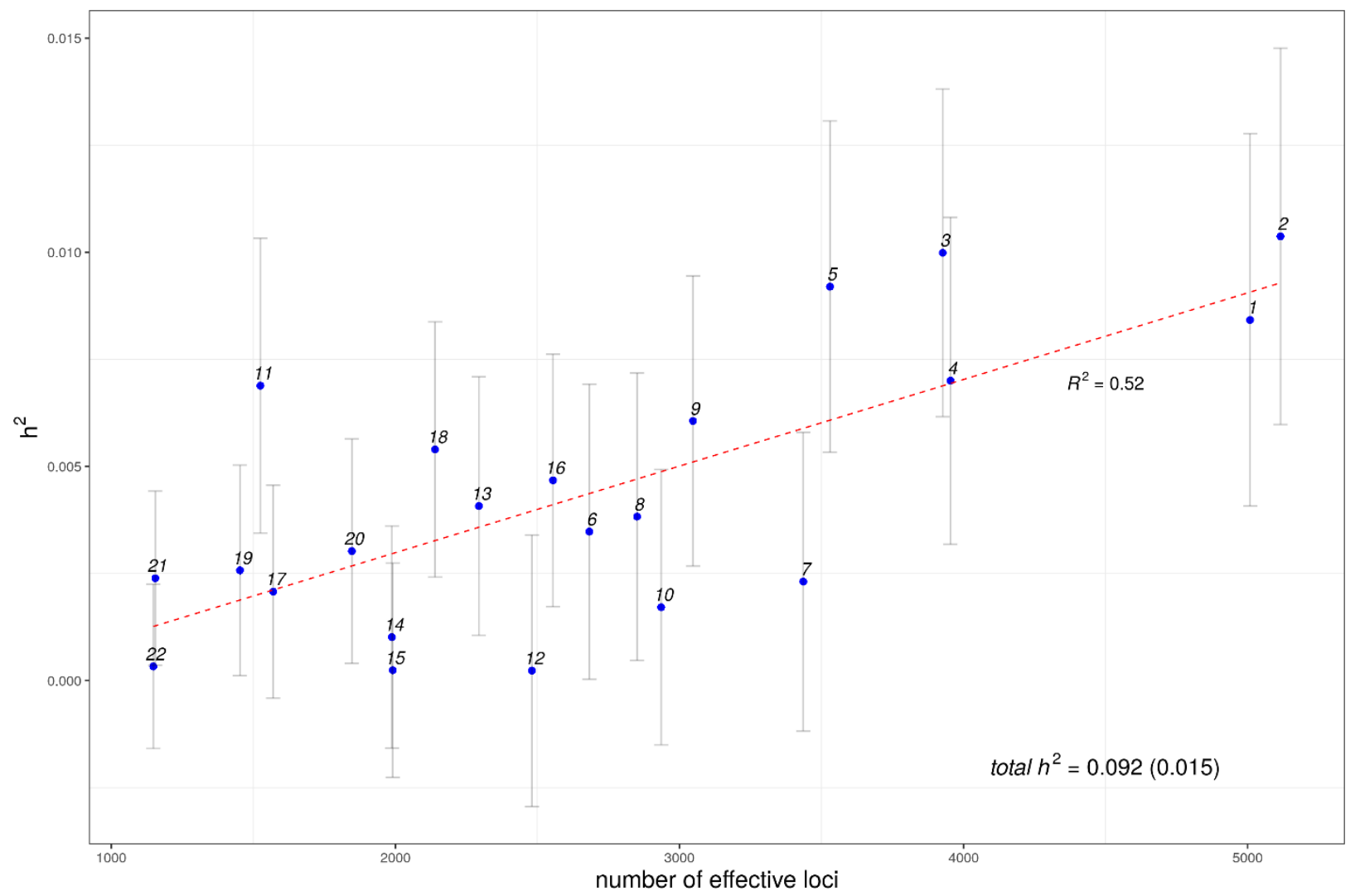

Figure A7. Decomposition of the total heritability of occupational wages by chromosomes

Note: GREML estimation in 24,000 unrelated individuals from the UK Biobank. Error bars are standard errors from GREML

estimation. The standardized coefficient of a regression of chromosomal $h^{2} \mathrm{sNP}$ on the effective number of loci per chromosome is 0.72 $(95 \%$ CI: $0.41-1.02)$. 


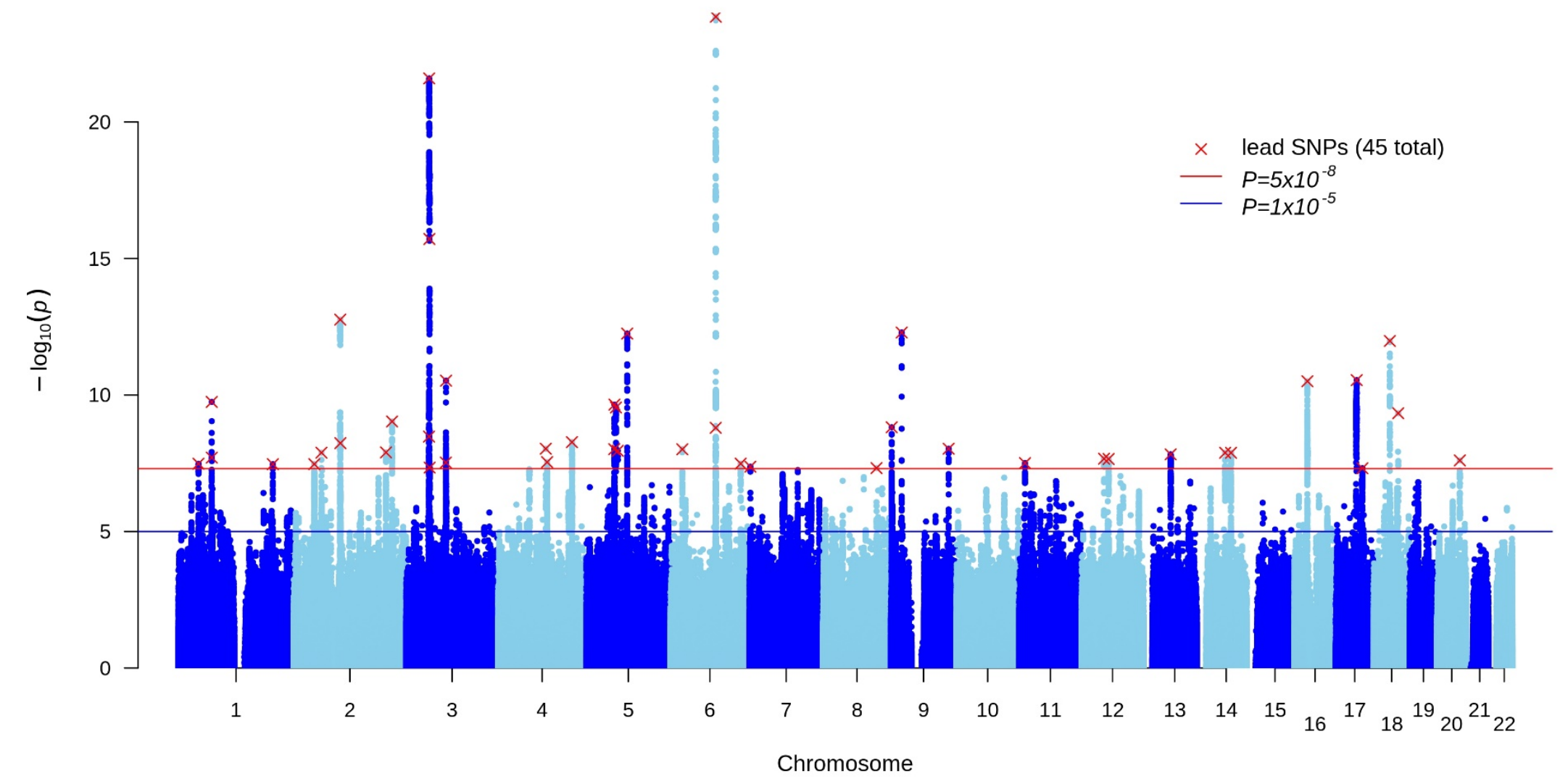

Figure A8. Manhattan plot for a genome-wide association study (GWAS) of occupational wages in the UK Biobank

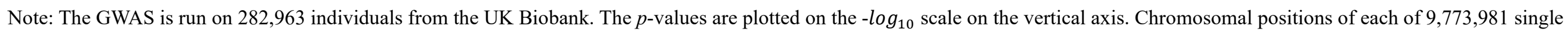
nucleotide polymorphisms (SNPs) are plotted on the horizontal axis. 
Table A1. List of variables in the UK Biobank

\begin{tabular}{|c|c|}
\hline Variables & Details \\
\hline log hourly wage & Imputed from standard occupation codes. See Appendix \\
\hline top household income & $=1$ if the annual household income before tax is greater than $£ 52,000$. \\
\hline log regional income & $\begin{array}{l}\text { Computed by matching home locations to Middle-layer Super Output Areas. Data from } \\
\text { Official National Statistics (England and Wales only) }\end{array}$ \\
\hline neighborhood score & $\begin{array}{l}\text { Negative of index of multiple deprivation for Lower-layer Super Output Areas derived by } \\
\text { the UK's Ministry of Housing, Communities \& Local Government (England only) }\end{array}$ \\
\hline years of education & See Okbay et al. (2016) \\
\hline college degree & $=1$ if having a college degree \\
\hline waist-to-hip ratio & Waist circumference divided by hip circumference \\
\hline BMI & Body mass index \\
\hline blood pressure & Average of systolic and diastolic measures \\
\hline lung function & $\begin{array}{l}\text { Average of forced expiratory volume in the first second, forced vital capacity, and peak } \\
\text { expiratory flow weighted by their covariance matrix. See Barcellos et al. (2019). }\end{array}$ \\
\hline ever hospitalized & $=1$ if any record in in-patient data \\
\hline ever diagnosed with cancer & $=1$ if any record in cancer registry \\
\hline infectious and parasitic diseases & $=1$ if any record for disorders classified in ICD-10 chapter 1 and codes Z20-29 \\
\hline neoplasms & $=1$ if any record for disorders classified in ICD-10 chapter 2 \\
\hline diseases of blood organs and immune system & $=1$ if any record for disorders classified in ICD-10 chapter 3 \\
\hline $\begin{array}{l}\text { endocrine, nutritional, and metabolic } \\
\text { diseases }\end{array}$ & $=1$ if any record for disorders classified in ICD-10 chapter 4 \\
\hline mental, behavioral, nervous system disorders & $=1$ if any record for disorders classified in ICD-10 chapter 5 and 6 \\
\hline diseases of the eye and adnexa & $=1$ if any record for disorders classified in ICD-10 chapter 7 \\
\hline diseases of the circulatory system & $=1$ if any record for disorders classified in ICD-10 chapter 9 \\
\hline diseases of the respiratory system & $=1$ if any record for disorders classified in ICD- 10 chapter 10 \\
\hline
\end{tabular}




\section{diseases of the digestive system}

diseases of the skin and subcutaneous tissue

diseases of musculoskeletal system and

connective tissue

diseases of genitourinary system

symptoms and signs not elsewhere classified

injury, poisoning, and other consequences of external causes

external causes of morbidity and mortality

other health conditions
$=1$ if any record for disorders classified in ICD-10 chapter 11

$=1$ if any record for disorders classified in ICD-10 chapter 12

$=1$ if any record for disorders classified in ICD-10 chapter 13

$=1$ if any record for disorders classified in ICD-10 chapter 14

$=1$ if any record for disorders classified in ICD-10 chapter 18

$=1$ if any record for disorders classified in ICD-10 chapter 19

$=1$ if any record for disorders classified in ICD-10 chapter 20

$=1$ if any record for the rest of disorders classified in ICD-10 
Table A2. Descriptive statistics

\begin{tabular}{|c|c|c|c|c|c|}
\hline & $\mathbf{N}$ & Mean & S.D & Min & Max \\
\hline \multicolumn{6}{|l|}{ UK Biobank } \\
\hline year of birth & 38697 & 1951.045 & 7.347 & 1937 & 1969 \\
\hline male & 38697 & 0.421 & 0.494 & 0 & 1 \\
\hline log hourly wage & 25292 & 2.6 & 0.349 & 1.833 & 3.881 \\
\hline top household income & 33477 & 0.233 & 0.423 & 0 & 1 \\
\hline regional income & 35393 & 739.482 & 193.387 & 300 & 1730 \\
\hline neighborhood score & 33276 & -17.646 & 13.937 & -82 & -0.61 \\
\hline years of education & 38424 & 14.627 & 5.135 & 7 & 20 \\
\hline college degree & 38424 & 0.291 & 0.454 & 0 & 1 \\
\hline waist-to-hip ratio & 38638 & 0.866 & 0.089 & 0.581 & 1.342 \\
\hline BMI & 38596 & 27.267 & 4.706 & 13.789 & 63.809 \\
\hline blood pressure & 36099 & 110.879 & 13.898 & 65.5 & 179 \\
\hline lung function & 35357 & 0 & 0.849 & -3.922 & 20.781 \\
\hline ever hospitalized & 38697 & 0.831 & 0.375 & 0 & 1 \\
\hline ever diagnosed with cancer & 38697 & 0.183 & 0.387 & 0 & 1 \\
\hline infectious and parasitic diseases & 38697 & 0.098 & 0.297 & 0 & 1 \\
\hline neoplasms & 38697 & 0.176 & 0.381 & 0 & 1 \\
\hline diseases of blood organs and immune system & 38697 & 0.222 & 0.416 & 0 & 1 \\
\hline endocrine, nutritional, and metabolic diseases & 38697 & 0.216 & 0.412 & 0 & 1 \\
\hline mental, behavioral, nervous system disorders & 38697 & 0.186 & 0.389 & 0 & 1 \\
\hline diseases of the eye and adnexa & 38697 & 0.117 & 0.322 & 0 & 1 \\
\hline diseases of the circulatory system & 38697 & 0.359 & 0.48 & 0 & 1 \\
\hline diseases of the respiratory system & 38697 & 0.172 & 0.378 & 0 & 1 \\
\hline diseases of the digestive system & 38697 & 0.426 & 0.494 & 0 & 1 \\
\hline diseases of the skin and subcutaneous tissue & 38697 & 0.118 & 0.323 & 0 & 1 \\
\hline diseases of musculoskeletal system and connective tissue & 38697 & 0.316 & 0.465 & 0 & 1 \\
\hline diseases of genitourinary system & 38697 & 0.283 & 0.45 & 0 & 1 \\
\hline symptoms and signs not elsewhere classified & 38697 & 0.389 & 0.487 & 0 & 1 \\
\hline injury, poisoning, and other consequences of external causes & 38697 & 0.171 & 0.376 & 0 & 1 \\
\hline external causes of morbidity and mortality & 38697 & 0.181 & 0.385 & 0 & 1 \\
\hline other health conditions & 38697 & 0.494 & 0.5 & 0 & 1 \\
\hline \multicolumn{6}{|l|}{ Health and Retirement Study } \\
\hline number of survey responses & 6171 & 3.605 & 2.047 & 1 & 12 \\
\hline year of birth & 6171 & 1944.487 & 8.431 & 1928 & 1968 \\
\hline male & 6171 & 0.451 & 0.498 & 0 & 1 \\
\hline hourly earnings & 22247 & 11.622 & 15.908 & 0.002 & 1244.848 \\
\hline
\end{tabular}


Table A2. Descriptive statistics

\begin{tabular}{lccccc}
\hline & N & Mean & S.D & Min & Max \\
\hline job experience & 22180 & 36.37 & 5.531 & 7 & 57 \\
years of education & 6152 & 13.588 & 2.414 & 0 & 17 \\
college degree & 6152 & 0.307 & 0.461 & 0 & 1 \\
father's years of education & 5635 & 10.667 & 3.538 & 0 & 17 \\
mother's yeras of education & 5862 & 11.06 & 2.918 & 0 & 17 \\
Wisconsin Longitudinal Study & & & & & \\
year of birth & 7387 & 39.742 & 3.747 & 29 & 64 \\
male & 7387 & 0.494 & 0.5 & 0 & 1 \\
sibling respondent & 7387 & 0.319 & 0.466 & 0 & 1 \\
hourly earnings & 7387 & 18.261 & 33.418 & 0.04 & 1282.051 \\
years of education & 6902 & 13.905 & 2.423 & 7 & 21 \\
\hline
\end{tabular}


Table A3. Summary of the UK Biobank samples

\begin{tabular}{|c|c|c|c|}
\hline Analysis & Sample & $\mathbf{N}$ & Note \\
\hline \multirow[t]{2}{*}{ Heritability } & Single-variance-parameter model & 196,187 & unrelated, wage observed \\
\hline & Multiple-variance-parameter model & 24,000 & randomly selected, unrelated, wage observed \\
\hline \multirow[t]{4}{*}{ GWAS } & Baseline sample & 282,963 & occupational wage observed \\
\hline & Two sub-samples & 141,481 each & $\begin{array}{l}\text { for GIV prediction, occupational wage observed, no relatives across the sub- } \\
\text { samples }\end{array}$ \\
\hline & Baseline sample without siblings and their relative & 252,958 & for prediction with the sibling sample, wage observed \\
\hline & Two sub-samples without siblings and their relative & 126,478 each & $\begin{array}{l}\text { for GIV prediction with the sibling sample, wage observed, no relatives across } \\
\text { the sub-samples }\end{array}$ \\
\hline Prediction & Sibling sample & 38,698 & 18,807 genetic sibling groups for within-family prediction analyses \\
\hline
\end{tabular}


Table A4. Weights used to construct the summary index of health in the UK Biobank sample

\begin{tabular}{lc}
\hline Health measure & Weight \\
\hline waist-to-hip ratio & 0.070 \\
BMI & 0.055 \\
blood pressure & 0.113 \\
lung function & 0.079 \\
ever hospitalized & 0.001 \\
ever diagnosed with cancer & 0.049 \\
infectious and parasitic diseases & 0.050 \\
neoplasms & 0.037 \\
diseases of blood organs and immune system & 0.062 \\
endocrine, nutritional, and metabolic diseases & -0.003 \\
mental, behavioral, nervous system disorders & 0.050 \\
diseases of the eye and adnexa & 0.101 \\
diseases of the circulatory system & -0.026 \\
diseases of the respiratory system & 0.041 \\
diseases of the digestive system & 0.044 \\
diseases of the skin and subcutaneous tissue & 0.080 \\
diseases of musculoskeletal system and connective tissue & 0.042 \\
diseases of genitourinary system & 0.077 \\
symptoms and signs not elsewhere classified & 0.019 \\
injury, poisoning, and other consequences of external causes & 0.065 \\
external causes of morbidity and mortality & 0.010 \\
other health conditions & -0.016 \\
\hline
\end{tabular}


Table A5. Heritability estimation of occupational wages in the UK Biobank using a

single variance genetic relatedness matrix restricted maximum likelihood (GREML)

\begin{tabular}{lcc}
\hline & Estimates & $N$ \\
\hline Male+Female & $0.103(0.003)$ & 196,187 \\
Male & $0.103(0.005)$ & 93,666 \\
Female & $0.126(0.005)$ & 102,521 \\
\hline
\end{tabular}

Note: Standard errors in parentheses. 


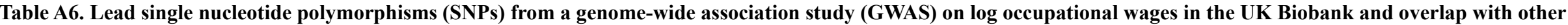
traits.

\begin{tabular}{|c|c|c|c|c|c|}
\hline SNP ID & $\begin{array}{l}\text { Chrom } \\
\text { o-some }\end{array}$ & $\begin{array}{l}\text { Effect- } \\
\text { coded } \\
\text { allele }\end{array}$ & $\begin{array}{c}\text { Effect } \\
\text { allele } \\
\text { frequency }\end{array}$ & Beta $\quad R^{2}(\%)$ & Overlap with other traits \\
\hline
\end{tabular}

rs 1487441

0.485

0.0097

0.037

Alcohol consumption (drinks per week), Autism and educational attinnent, Bipolar disorder, Cognitive ability,

Educational attainment, Extremely high intelligence, General risk tolerance, Highest math class taken, Household

income, Intelligence, QT interval (drug interaction), Regular attendance at a pub or social club, Risk-taking tendency (4domain principal component model), Self-reported math ability, Tourette syndrome

Blood protein levels, Chronic inflammatory diseases (ankylosing spondylitis, Crohn's disease, psoriasis, primary sclerosing cholangitis, ulcerative colitis) (pleiotropy), Cognitive ability, Crohn's disease, Depressed affect, Educational attainment, Estimated glomerular filtration rate, Extremely high intelligence, Feeling fed-up, Glioblastoma, Glioma, Gut microbiota (functional units), Highest math class taken, Household income, Inflammatory bowel disease, Intelligence, Menarche (age at onset), Metabolite levels, Parental longevity (father's age at death or father's attained age), Pediatric autoimmune diseases, Primary sclerosing cholangitis, Regular attendance at a religious group, Self-reported math ability, Ulcerative colitis

BMI, Cognitive ability, Cognitive ability, years of educational attainment or schizophrenia (pleiotropy), Depressed affect, Educational attainment, Feeling miserable, Gastroesophageal reflux disease, HDL cholesterol, HDL cholesterol levels, HDL cholesterol levels in current drinkers, HDL cholesterol levels x alcohol consumption (drinkers vs nondrinkers) interaction (2df), HDL cholesterol levels x alcohol consumption (regular vs non-regular drinkers) interaction (2df), HDL cholesterol x physical activity interaction (2df test), Household income, Intelligence, Menarche (age at onset), Morning person, Morningness, Parental longevity (mother's age at death or mother's attained age), Predicted visceral adipose tissue, Regular attendance at a gym or sports club, White blood cell count Extremely high intelligence, Feeling lonely, Highest math class taken, Household income, Insomnia symptoms (never/rarely vs. sometimes/usually), Insomnia symptoms (never/rarely vs. usually), Intelligence, Mental health study participation (completed survey), Remission after SSRI treatment in MDD or neuroticism, Self-reported math ability

Cognitive ability, Depression, Educational attainment, Highest math class taken, Household income, Intelligence, Lung function (FVC), Major depressive disorder, Sedentary behaviour duration, Self-reported math ability

Cognitive ability, Cognitive ability, years of educational attainment or schizophrenia (pleiotropy), Depressed affect,

Depression, Depression (broad), Educational attainment, Experiencing mood swings, Feeling fed-up, Feeling hurt, General factor of neuroticism, Highest math class taken, Household income, Lifetime smoking index, Mood instability, Neuroticism, Neuroticism, Remission after SSRI treatment in MDD or neuroticism, Response to amphetamines, Subjective well-being, Well-being spectrum (multivariate analysis), Worry

Alcohol consumption (drinks per week), Alzheimer's disease in APOE e4- carriers, Balding type 1, Brain region volumes, Breast cancer, Celiac disease, Cognitive ability, Depressed affect, Epithelial ovarian cancer, Experiencing mood swings, Feeling fed-up, Feeling guilty, Feeling hurt, Feeling miserable, Feeling nervous, Feeling worry, General mood swings, Feeling fed-up, Feeling guilty, Feeling hurt, Feeling miserable, Feeling nervous, Feeling worry, Gen
factor of neuroticism, Handedness (Left-handed vs. non-left-handed), Handedness (left-handed vs. right-handed), Handedness (non-right-handed vs right-handed), Intelligence, Intracranial volume, Intraocular pressure, Irritable mood, Lung function (FEV1), Lung function (FVC), Macular thickness, Male-pattern baldness, Multiple system atrophy, Neuroticism, Neuroticism, Ovarian cancer in BRCA1 mutation carriers, Parkinson's disease, Parkinson's disease or first 

microstructure (axial diusivities), White matter microstructure (fractional anisotropy), White matter microstructure (mean diusivities), White matter microstructure (radial diusivities), Worry

BMI, General risk tolerance, Household income, Predicted visceral adipose tissue, Risk-taking tendency (4-domain principal component model), Smoking initiation (ever regular vs never regular), Smoking status, Smoking status (ever vs never smokers), Waist circumference, Waist-hip ratio

Albumin-globulin ratio, Alcohol consumption (drinks per week), Allergic disease (asthma, hay fever or eczema), Bipolar disorder or body mass index, Blood protein levels, BMI, BMI (joint analysis main effects and physical activity interaction), BMI in physically active individuals, Body fat percentage, Childhood obesity, Chronic inflammatory diseases (ankylosing spondylitis, Crohn's disease, psoriasis, primary sclerosing cholangitis, ulcerative colitis) (pleiotropy), Chronic obstructive pulmonary disease, Cognitive ability, Crohn's disease, Eczema, Educational attainment, Estimated glomerular filtration rate, Extremely high intelligence, Hand grip strength, Hip circumference, Hip circumference adjusted for BMI, Household income, Inflammatory bowel disease, Inflammatory bowel disease (early onset), Intelligence, Offspring birth weight, Pediatric autoimmune diseases, Red blood cell count, Red cell distribution width, Type 1 diabetes, Ulcerative colitis, Waist circumference, Weight

\begin{tabular}{|c|c|c|c|c|c|c|}
\hline rs 34305371 & 1 & A & 0.102 & 0.0100 & 0.014 & $\begin{array}{l}\text { Alcohol consumption (drinks per week), Cognitive ability, Cognitive ability, years of educational attainment or } \\
\text { schizophrenia (pleiotropy), Educational attainment, Household income, Insomnia, Intelligence }\end{array}$ \\
\hline rs 78871889 & 5 & $\mathrm{~T}$ & 0.299 & -0.0066 & 0.014 & Educational attainment, Height, Highest math class taken, Household income \\
\hline rs 10053567 & 5 & $\mathrm{C}$ & 0.218 & 0.0072 & 0.014 & Educational attainment \\
\hline rs619466 & 18 & G & 0.906 & -0.0101 & 0.014 & $\begin{array}{l}\text { Automobile speeding propensity, Depressed affect, Depressive symptoms, General factor of neuroticism, Household } \\
\text { income, Life satisfaction, Neuroticism, Positive affect, Well-being spectrum (multivariate analysis) }\end{array}$ \\
\hline rs62183028 & 2 & $\mathrm{~T}$ & 0.312 & -0.0063 & 0.013 & $\begin{array}{l}\text { Cognitive ability, Depressed affect, Educational attainment, Highest math class taken, Household income, Intelligence, } \\
\text { Self-reported math ability }\end{array}$ \\
\hline rs3847223 & 9 & $\mathrm{~T}$ & 0.523 & 0.0057 & 0.013 & Educational attainment, Household income \\
\hline rs6928545 & 6 & G & 0.317 & -0.0061 & 0.013 & Cognitive ability, Household income, Intelligence \\
\hline rs113011189 & 3 & $\mathrm{~T}$ & 0.091 & -0.0097 & 0.012 & Educational attainment \\
\hline rs59971723 & 4 & A & 0.381 & -0.0057 & 0.012 & $\begin{array}{l}\text { Cognitive ability, Cognitive ability, years of educational attainment or schizophrenia (pleiotropy), Educational } \\
\text { attainment, Height, Highest math class taken, Household income, Intelligence }\end{array}$ \\
\hline rs56211325 & 2 & $\mathrm{~T}$ & 0.024 & 0.0182 & 0.012 & Cognitive ability, Household income, Intelligence \\
\hline rs 13107325 & 4 & $\mathrm{~T}$ & 0.074 & -0.0104 & 0.012 & $\begin{array}{l}\text { Adventurousness, Alcohol consumption, Alcohol consumption (drinks per week), Alcohol use disorder, Alcohol use } \\
\text { disorder (consumption score), Alcohol use disorder (dependence and problematic use scores), Alcohol use disorder (total } \\
\text { score), Autism spectrum disorder or schizophrenia, Balding type 1, Bitter alcoholic beverage consumption, Blood } \\
\text { pressure, BMI, BMI (adjusted for smoking behaviour), BMI (joint analysis main effects and physical activity } \\
\text { interaction), BMI (joint analysis main effects and smoking interaction), BMI in non-smokers, BMI in physically active } \\
\text { individuals, Body fat percentage, Brain imaging measurements, Brain region volumes, Childhood body mass index, } \\
\text { Cognitive ability, Cognitive ability, years of educational attainment or schizophrenia (pleiotropy), Diastolic blood } \\
\text { pressure, Diastolic blood pressure x alcohol consumption interaction (2df test), Eczema, Educational attainment, Hand } \\
\text { grip strength, HDL cholesterol, HDL cholesterol levels, HDL cholesterol levels in current drinkers, HDL cholesterol } \\
\text { levels x alcohol consumption (drinkers vs non-drinkers) interaction (2df), HDL cholesterol levels x alcohol consumption }\end{array}$ \\
\hline
\end{tabular}




\begin{tabular}{|c|c|c|c|c|c|c|}
\hline rs10818605 & 9 & $\mathrm{~T}$ & 0.556 & 0.0055 & 0.012 & Cognitive ability, Educational attainment, Highest math class taken, Household income \\
\hline rs 7715147 & 5 & A & 0.242 & 0.0063 & 0.012 & Household income, Multiple system atrophy \\
\hline rs6935954 & 6 & G & 0.576 & 0.0055 & 0.012 & $\begin{array}{l}\text { Age of smoking initiation, Birth weight, Chronotype, Educational attainment, Height, Lifetime smoking index, } \\
\text { Mosquito bite size, Offspring birth weight, Parental longevity (father's age at death or father's attained age), Smoking } \\
\text { cessation }\end{array}$ \\
\hline rs 10515086 & 5 & $\mathrm{~T}$ & 0.171 & -0.0072 & 0.012 & Educational attainment, Household income \\
\hline rs 1455351 & 2 & G & 0.418 & -0.0055 & 0.011 & $\begin{array}{l}\text { Cognitive ability, Educational attainment, Experiencing mood swings, Highest math class taken, Household income, } \\
\text { Inflammatory bowel disease, Intelligence, Mood instability, Self-reported math ability, Smoking initiation (ever regular } \\
\text { vs never regular), Smoking status, Ulcerative colitis }\end{array}$ \\
\hline rs 7556782 & 2 & $\mathrm{C}$ & 0.634 & -0.0056 & 0.011 & $\begin{array}{l}\text { Carpal tunnel syndrome, Risk-taking tendency (4-domain principal component model), Smoking cessation, Waist-hip } \\
\text { ratio }\end{array}$ \\
\hline rs 1254319 & 14 & A & 0.294 & 0.0059 & 0.011 & $\begin{array}{l}\text { Chronotype, Educational attainment, Glaucoma, Glaucoma (high intraocular pressure), Glaucoma (primary open-angle), } \\
\text { Heel bone mineral density, Height, Highest math class taken, Hip circumference adjusted for BMI, Medication use } \\
\text { (antiglaucoma preparations and miotics), Menarche (age at onset), Optic cup area, Optic disc area, Optic disc size, Optic } \\
\text { nerve measurement (rim area), Refractive error, Self-reported math ability, Vertical cup-disc ratio, Waist-hip ratio }\end{array}$ \\
\hline rs 2535911 & 14 & $\mathrm{~T}$ & 0.357 & 0.0056 & 0.011 & Cognitive ability \\
\hline rs6561943 & 13 & $\mathrm{~T}$ & 0.257 & -0.0061 & 0.011 & $\begin{array}{l}\text { BMI, Cognitive ability, Cognitive ability, years of educational attainment or schizophrenia (pleiotropy), Educational } \\
\text { attainment, Experiencing mood swings, Highest math class taken, Household income, Intelligence, Predicted visceral } \\
\text { adipose tissue, Type } 2 \text { diabetes }\end{array}$ \\
\hline rs10789336 & 1 & A & 0.597 & 0.0054 & 0.011 & $\begin{array}{l}\text { Allergic rhinitis, Asthma and major depressive disorder, BMI, BMI (adjusted for smoking behaviour), BMI (joint } \\
\text { analysis main effects and physical activity interaction), BMI (joint analysis main effects and smoking interaction), BMI } \\
\text { in non-smokers, BMI in physically active individuals, BMI in physically inactive individuals, BMI in smokers, Body fat } \\
\text { percentage, Childhood body mass index, Cognitive ability, Depression, Depression (broad), Depressive symptoms, Hip } \\
\text { circumference, Insomnia, Intelligence, Major depressive disorder, Menarche (age at onset), Obesity, Obesity (early onset } \\
\text { extreme), Subcutaneous adipose tissue, Waist circumference, Weight }\end{array}$ \\
\hline rs11168416 & 12 & $\mathrm{~T}$ & 0.324 & -0.0057 & 0.011 & Educational attainment, Highest math class taken, Metabolite levels \\
\hline rs7975227 & 12 & G & 0.371 & -0.0055 & 0.011 & Intelligence \\
\hline rs4811076 & 20 & G & 0.511 & 0.0053 & 0.011 & $\begin{array}{l}\text { Cognitive ability, Educational attainment, Highest math class taken, Self-reported math ability, Vitiligo, White blood cell } \\
\text { count }\end{array}$ \\
\hline
\end{tabular}

(regular vs non-regular drinkers) interaction (2df), HDL cholesterol x physical activity interaction (2df test), Height,

High density lipoprotein cholesterol levels, Hypertension, Insomnia, Intelligence, Low density lipoprotein cholesterol levels, Lung function (FVC), Male-pattern baldness, Mean arterial pressure, Mean arterial pressure x alcohol consumption interaction (2df test), Medication use (agents acting on the renin-angiotensin system), Multisite chronic pain, NT-proBNP levels in acute coronary syndrome, Obese vs. thin, Osteoarthritis, Predicted visceral adipose tissue, Red blood cell count, Risk-taking tendency (4-domain principal component model), Schizophrenia, Self-reported math ability, Sleep duration (short sleep), Systolic blood pressure, Systolic blood pressure x alcohol consumption interaction (2df test), Total cholesterol levels, Voxel-wise structural brain imaging measurements, vWF levels, Waist-hip ratio, Waist-to-hip ratio adjusted for BMI, White blood cell count 


\begin{tabular}{|c|c|c|c|c|c|c|}
\hline rs 2726518 & 4 & $\mathrm{C}$ & 0.571 & -0.0053 & 0.011 & $\begin{array}{l}\text { Adventurousness, Cognitive ability, Colorectal cancer or advanced adenoma, Educational attainment, Highest math class } \\
\text { taken, Household income, Intelligence, Multiple sclerosis }\end{array}$ \\
\hline rs9824386 & 3 & A & 0.865 & 0.0077 & 0.011 & $\begin{array}{l}\text { Chronotype, Cognitive ability, Educational attainment, Highest math class taken, Intelligence, Metabolite levels, } \\
\text { Morningness }\end{array}$ \\
\hline rs7940022 & 11 & $\mathrm{~T}$ & 0.680 & 0.0056 & 0.011 & $\begin{array}{l}\text { Balding type 1, Cognitive ability, Cognitive ability, years of educational attainment or schizophrenia (pleiotropy), } \\
\text { Educational attainment, Highest math class taken, Household income, Intelligence, Male-pattern baldness }\end{array}$ \\
\hline rs12204714 & 6 & $\mathrm{~T}$ & 0.632 & 0.0054 & 0.011 & Age at first birth, Educational attainment \\
\hline rs2494995 & 1 & $\mathrm{C}$ & 0.217 & -0.0063 & 0.011 & $\begin{array}{l}\text { Adventurousness, Attention deficit hyperactivity disorder or cannabis use, Balding type } 1 \text {, Coffee consumption, } \\
\text { Cognitive ability, Educational attainment, Household income, Intelligence, Number of sexual partners, Perceived } \\
\text { intensity of sweet substances }\end{array}$ \\
\hline rs4953097 & 2 & $\mathrm{~T}$ & 0.663 & -0.0055 & 0.011 & Age at first sexual intercourse, Chronotype, Educational attainment, General risk tolerance, Height, Morning person \\
\hline rs9729959 & 1 & $\mathrm{~T}$ & 0.226 & 0.0062 & 0.011 & $\begin{array}{l}\text { Cognitive ability, Cognitive ability, years of educational attainment or schizophrenia (pleiotropy), Educational } \\
\text { attainment, Glioma, Highest math class taken, Household income, Intelligence, Non-glioblastoma glioma, Regular } \\
\text { attendance at a religious group, Self-reported math ability, Urinary sodium excretion }\end{array}$ \\
\hline rs 13240401 & 7 & $\mathrm{C}$ & 0.224 & -0.0062 & 0.011 & Cognitive ability, Educational attainment, Highest math class taken, Self-reported math ability \\
\hline rs 2282760 & 3 & G & 0.132 & -0.0076 & 0.011 & $\begin{array}{l}\text { Age at voice drop, Coffee consumption, Depressive symptom (appetite changes) (binary trait), Depressive symptom } \\
\text { (fatigue) (ordinal trait), Depressive symptoms (binary sum-score), Depressive symptoms (sum-score), Diastolic blood } \\
\text { pressure, Eosinophil counts, Lung function (FVC), Morningness, Systolic blood pressure, Waist-to-hip ratio adjusted for } \\
\text { BMI (additive genetic model) }\end{array}$ \\
\hline rs 141349367 & 8 & $\mathrm{~T}$ & 0.059 & -0.0109 & 0.011 & \\
\hline rs4408596 & 17 & G & 0.623 & 0.0053 & 0.011 & Cognitive ability, Intelligence, Morning person \\
\hline
\end{tabular}

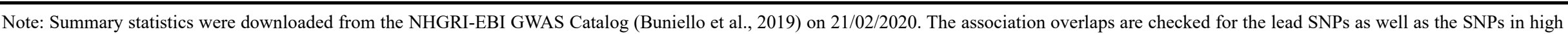

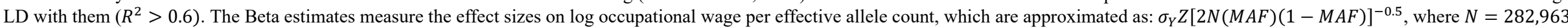

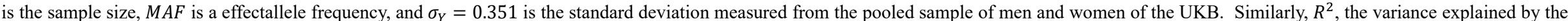
SNP alone, is approximated as $Z^{2} / N$. 
Table A7. Associations between polygenic indices for income and self-reported wages in the Health and Retirement Study and the Wisconsin Longitudinal Study

\begin{tabular}{|c|c|c|c|c|c|c|c|c|c|c|c|c|}
\hline & \multicolumn{4}{|c|}{ Male } & \multicolumn{4}{|c|}{ Female } & \multicolumn{4}{|c|}{ Male+Female } \\
\hline \multicolumn{13}{|c|}{ Panel A: Health and Retirement Study } \\
\hline Income PGI & & $\begin{array}{c}0.083 * * * \\
(0.014)\end{array}$ & & $\begin{array}{c}0.047 * * \\
(0.017)\end{array}$ & & $\begin{array}{c}0.078 * * * \\
(0.011)\end{array}$ & & $\begin{array}{c}0.043 * * \\
(0.014)\end{array}$ & & $\begin{array}{c}0.080 * * * \\
(0.009)\end{array}$ & & $\begin{array}{c}0.045 * * * \\
(0.011)\end{array}$ \\
\hline EA PGI & & & $\begin{array}{c}0.093 * * * \\
(0.013)\end{array}$ & $\begin{array}{c}0.065 * * * \\
(0.017)\end{array}$ & & & $\begin{array}{c}0.089 * * * \\
(0.011)\end{array}$ & $\begin{array}{c}0.065^{* * *} \\
(0.014)\end{array}$ & & & $\begin{array}{c}0.091 * * * \\
(0.009)\end{array}$ & $\begin{array}{c}0.065 * * * \\
(0.011)\end{array}$ \\
\hline$R^{2}(\%)$ & 2.201 & 3.192 & 3.368 & 3.585 & 4.152 & 5.086 & 5.304 & 5.509 & 8.625 & 9.531 & 9.720 & 9.919 \\
\hline$\Delta R^{2}(\%)$ & & 0.991 & 1.167 & 1.384 & & 0.934 & 1.152 & 1.358 & & 0.905 & 1.095 & 1.294 \\
\hline partial $R^{2}(\%)$ & & 1.013 & 1.194 & 1.415 & & 0.975 & 1.202 & 1.416 & & 0.991 & 1.198 & 1.416 \\
\hline Obs. & 9183 & & & & 13064 & & & & 22247 & & & \\
\hline \multicolumn{13}{|c|}{ Panel B: Wisconsin Longitudinal Study } \\
\hline Income PGI & & $\begin{array}{c}0.048 * * * \\
(0.012)\end{array}$ & & $\begin{array}{c}0.024 \\
(0.014)\end{array}$ & & $\begin{array}{c}0.068^{* * *} \\
(0.011)\end{array}$ & & $\begin{array}{c}0.048 * * * \\
(0.014)\end{array}$ & & $\begin{array}{c}0.058 * * * \\
(0.008)\end{array}$ & & $\begin{array}{c}0.036^{* * * *} \\
(0.010)\end{array}$ \\
\hline EA PGI & & & $\begin{array}{c}0.060 * * * \\
(0.012)\end{array}$ & $\begin{array}{c}0.047 * * * \\
(0.014)\end{array}$ & & & $\begin{array}{c}0.063 * * * \\
(0.011)\end{array}$ & $\begin{array}{c}0.035^{* *} \\
(0.014)\end{array}$ & & & $\begin{array}{c}0.061 * * * \\
(0.008)\end{array}$ & $\begin{array}{c}0.041 * * * \\
(0.010)\end{array}$ \\
\hline$R^{2}(\%)$ & 2.082 & 2.560 & 2.785 & 2.873 & 1.915 & 2.859 & 2.704 & 3.035 & 19.172 & 19.743 & 19.787 & 19.943 \\
\hline$\Delta R^{2}(\%)$ & & 0.478 & 0.702 & 0.791 & & 0.944 & 0.789 & 1.120 & & 0.571 & 0.615 & 0.771 \\
\hline partial $R^{2}(\%)$ & & 0.488 & 0.717 & 0.807 & & 0.963 & 0.804 & 1.142 & & 0.706 & 0.761 & 0.954 \\
\hline Obs. & 3650 & & & & 3737 & & & & 7387 & & & \\
\hline
\end{tabular}


Table A8. Associations between polygenic indices for income and 3-year moving averages of self-reported wages in the Health and Retirement Study

\begin{tabular}{|c|c|c|c|c|c|c|c|c|c|c|c|c|}
\hline \multirow[b]{2}{*}{ Income PGI } & \multicolumn{4}{|c|}{ Male } & \multicolumn{4}{|c|}{ Female } & \multicolumn{4}{|c|}{ Male+Female } \\
\hline & & $\begin{array}{c}0.078 * * * \\
(0.016)\end{array}$ & & $\begin{array}{c}0.046^{*} \\
(0.020)\end{array}$ & & $\begin{array}{c}0.071 * * * \\
(0.013)\end{array}$ & & $\begin{array}{l}0.035^{*} \\
(0.015)\end{array}$ & & $\begin{array}{c}0.074 * * * \\
(0.010)\end{array}$ & & $\begin{array}{c}0.039 * * \\
(0.012)\end{array}$ \\
\hline EA PGI & & & $\begin{array}{c}0.088 * * * \\
(0.015)\end{array}$ & $\begin{array}{c}0.061 * * \\
(0.020)\end{array}$ & & & $\begin{array}{c}0.087 * * * \\
(0.013)\end{array}$ & $\begin{array}{c}0.067 * * * \\
(0.016)\end{array}$ & & & $\begin{array}{c}0.087 * * * \\
(0.010)\end{array}$ & $\begin{array}{c}0.065 * * * \\
(0.012)\end{array}$ \\
\hline$R^{2}(\%)$ & 4.087 & 5.675 & 5.900 & 6.263 & 5.981 & 7.409 & 7.992 & 8.243 & 13.476 & 14.834 & 15.239 & 15.506 \\
\hline$\Delta R^{2}(\%)$ & & 1.588 & 1.813 & 2.176 & & 1.427 & 2.010 & 2.262 & & 1.358 & 1.763 & 2.030 \\
\hline partial $R^{2}(\%)$ & & 1.655 & 1.89 & 2.269 & & 1.518 & 2.138 & 2.406 & & 1.57 & 2.038 & 2.346 \\
\hline Obs. & 4,144 & & & & 6,768 & & & & 10,912 & & & \\
\hline
\end{tabular}

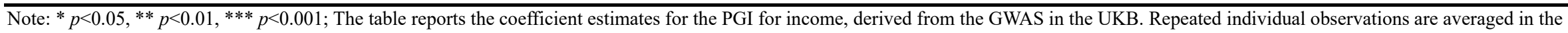

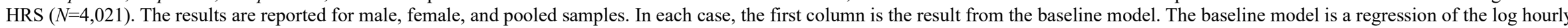

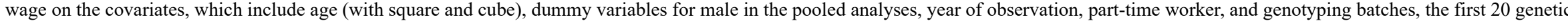

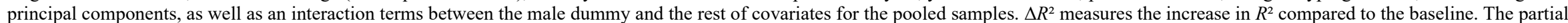

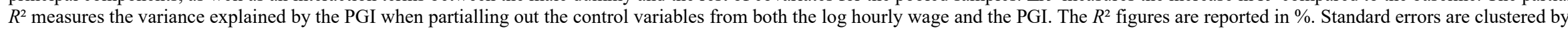
individuals and reported in parentheses. 
Table A9. Comparison of predictive power of polygenic indices in UK Biobank sibling sample.

\begin{tabular}{lccc}
\hline & Income PGI $\Delta R^{2}$ & MTAG PGI $\Delta R^{2}$ & $N$ \\
\hline Log occupational wage per hour & $2.77 \%$ & $4.47 \%$ & 17,690 \\
Years of education & $4.18 \%$ & $7.26 \%$ & 35,128 \\
BMI & $0.66 \%$ & $1.40 \%$ & 35,428 \\
\hline
\end{tabular}

Note: The table reports the change in $R^{2}$ when a polygenic indice is added to the model. As covariates, all analyses include dummy variables for the year of birth, male, and being the younger sibling as well as the first 20 genetic PCs. For occupational wages, we use age dummies instead of the year of birth and add dummies for the year of survey. For BMI ratios we also control for the age dummies instead but not for the year of survey. In every case, we also include the interaction terms between the male dummy and the rest of covariates. 
Table A10. Estimates of 3-year moving-average income with respect to schooling and genetic factors in the Health and Retirement Study

\begin{tabular}{|c|c|c|c|c|c|c|c|c|c|c|c|c|c|c|c|c|}
\hline \multicolumn{2}{|c|}{ Panel A: Male+Female } & \multicolumn{3}{|c|}{ no PGI } & \multicolumn{4}{|c|}{ naive control } & \multicolumn{4}{|c|}{ GIV-C } & \multicolumn{4}{|c|}{ GIV-U } \\
\hline Educ & $\begin{array}{c}0.1107 * * * \\
(0.008)\end{array}$ & $\begin{array}{c}0.1055 * * * \\
(0.008)\end{array}$ & $\begin{array}{c}0.0920^{* * * *} \\
(0.012)\end{array}$ & $\begin{array}{c}0.0857 * * * \\
(0.012)\end{array}$ & $\begin{array}{c}0.1076^{* * * *} \\
(0.008)\end{array}$ & $\begin{array}{c}0.1026^{* * * *} \\
(0.008)\end{array}$ & $\begin{array}{c}0.0900 * * * \\
(0.012)\end{array}$ & $\begin{array}{c}0.0840^{* * *} \\
(0.012)\end{array}$ & $\begin{array}{c}0.1048^{* * *} \\
(0.009)\end{array}$ & $\begin{array}{c}0.0997 * * * \\
(0.009)\end{array}$ & $\begin{array}{c}0.0890^{* * *} \\
(0.013)\end{array}$ & $\begin{array}{c}0.0826^{* * *} \\
(0.013)\end{array}$ & $\begin{array}{c}0.1040^{* * * *} \\
(0.009)\end{array}$ & $\begin{array}{c}0.0992 * * * \\
(0.009)\end{array}$ & $\begin{array}{c}0.0880^{* * * *} \\
(0.012)\end{array}$ & $\begin{array}{c}0.0821 * * * \\
(0.013)\end{array}$ \\
\hline Educ $\times$ College & & & $\begin{array}{l}0.0615 \\
(0.034)\end{array}$ & $\begin{array}{l}0.0603 \\
(0.034)\end{array}$ & & & $\begin{array}{l}0.0594 \\
(0.034)\end{array}$ & $\begin{array}{l}0.0584 \\
(0.034)\end{array}$ & & & $\begin{array}{l}0.0582 \\
(0.035)\end{array}$ & $\begin{array}{l}0.0571 \\
(0.035)\end{array}$ & & & $\begin{array}{l}0.0548 \\
(0.034)\end{array}$ & $\begin{array}{l}0.0541 \\
(0.034)\end{array}$ \\
\hline Income PGI & & & & & $\begin{array}{c}0.0284^{* *} \\
(0.009)\end{array}$ & $\begin{array}{c}0.0272 * * \\
(0.009)\end{array}$ & $\begin{array}{c}0.0272^{* *} \\
(0.009)\end{array}$ & $\begin{array}{c}0.0258^{* *} \\
(0.009)\end{array}$ & $\begin{array}{l}0.1224 * \\
(0.056)\end{array}$ & $\begin{array}{l}0.1138^{*} \\
(0.055)\end{array}$ & $\begin{array}{l}0.1184^{*} \\
(0.056)\end{array}$ & $\begin{array}{l}0.1092 * \\
(0.054)\end{array}$ & $\begin{array}{l}0.0663 * \\
(0.030)\end{array}$ & $\begin{array}{l}0.0623 * \\
(0.029)\end{array}$ & $\begin{array}{l}0.0641^{*} \\
(0.029)\end{array}$ & $\begin{array}{l}0.0598^{*} \\
(0.029)\end{array}$ \\
\hline $\begin{array}{l}\text { Parental Educ } \\
\mathrm{R}^{2}\end{array}$ & 0.299 & $\begin{array}{c}\mathrm{Y} \\
0.301\end{array}$ & 0.301 & $\begin{array}{c}\mathrm{Y} \\
0.303\end{array}$ & 0.301 & $\begin{array}{c}\mathrm{Y} \\
0.303\end{array}$ & 0.303 & $\begin{array}{c}\mathrm{Y} \\
0.305\end{array}$ & & $\mathrm{Y}$ & & $\mathrm{Y}$ & & $\mathrm{Y}$ & & Y \\
\hline Obs. & 9906 & - & - & - & - & - & - & - & - & - & - & - & - & - & - & - \\
\hline Panel B: Male & & & & & & & & & & & & & & & & \\
\hline Educ & $\begin{array}{c}0.0880 * * * \\
(0.013)\end{array}$ & $\begin{array}{c}0.0813 * * * \\
(0.013)\end{array}$ & $\begin{array}{c}0.0437 * * \\
(0.016)\end{array}$ & $\begin{array}{l}0.0378^{*} \\
(0.016)\end{array}$ & $\begin{array}{c}0.0859 * * * \\
(0.013)\end{array}$ & $\begin{array}{c}0.0795^{* * *} \\
(0.013)\end{array}$ & $\begin{array}{c}0.0434^{* *} \\
(0.016)\end{array}$ & $\begin{array}{l}0.0376^{*} \\
(0.016)\end{array}$ & $\begin{array}{c}0.0821^{* * *} \\
(0.014)\end{array}$ & $\begin{array}{c}0.0763 * * * \\
(0.014)\end{array}$ & $\begin{array}{c}0.0483 * * \\
(0.016)\end{array}$ & $\begin{array}{c}0.0427^{* *} \\
(0.016)\end{array}$ & $\begin{array}{c}0.0805^{* * *} \\
(0.013)\end{array}$ & $\begin{array}{c}0.0743^{* * *} \\
(0.014)\end{array}$ & $\begin{array}{c}0.0437 * * \\
(0.016)\end{array}$ & $\begin{array}{c}0.0380^{*} \\
(0.016)\end{array}$ \\
\hline Educ $\times$ College & & & $\begin{array}{l}0.0353 \\
(0.050)\end{array}$ & $\begin{array}{l}0.0343 \\
(0.050)\end{array}$ & & & $\begin{array}{l}0.0337 \\
(0.050)\end{array}$ & $\begin{array}{l}0.0329 \\
(0.050)\end{array}$ & & & $\begin{array}{l}0.0400 \\
(0.053)\end{array}$ & $\begin{array}{l}0.0390 \\
(0.053)\end{array}$ & & & $\begin{array}{l}0.0247 \\
(0.051)\end{array}$ & $\begin{array}{l}0.0240 \\
(0.051)\end{array}$ \\
\hline Income PGI & & & & & $\begin{array}{l}0.0261 \\
(0.016)\end{array}$ & $\begin{array}{l}0.0246 \\
(0.015)\end{array}$ & $\begin{array}{l}0.0208 \\
(0.015)\end{array}$ & $\begin{array}{l}0.0194 \\
(0.015)\end{array}$ & $\begin{array}{c}0.1986 * \\
(0.100)\end{array}$ & $\begin{array}{l}0.1934 \\
(0.100)\end{array}$ & $\begin{array}{l}0.1814 \\
(0.102)\end{array}$ & $\begin{array}{l}0.1758 \\
(0.101)\end{array}$ & $\begin{array}{c}0.1057^{*} \\
(0.051)\end{array}$ & $\begin{array}{c}0.1035^{*} \\
(0.051)\end{array}$ & $\begin{array}{l}0.0954 \\
(0.052)\end{array}$ & $\begin{array}{l}0.0930 \\
(0.052)\end{array}$ \\
\hline $\begin{array}{l}\text { Parental Educ } \\
\mathrm{R}^{2}\end{array}$ & 0.194 & $\begin{array}{c}\mathrm{Y} \\
0.198\end{array}$ & 0.203 & $\begin{array}{c}\mathrm{Y} \\
0.207\end{array}$ & 0.196 & $\begin{array}{c}\mathrm{Y} \\
0.199\end{array}$ & 0.204 & $\begin{array}{c}\mathrm{Y} \\
0.208\end{array}$ & & $\mathrm{Y}$ & & $\mathrm{Y}$ & & $\mathrm{Y}$ & & Y \\
\hline Obs. & 3784 & - & - & - & - & - & - & - & - & - & - & - & - & - & - & - \\
\hline Panel C: Female & & & & & & & & & & & & & & & & \\
\hline Educ & $\begin{array}{c}0.1248 * * * \\
(0.011)\end{array}$ & $\begin{array}{c}0.1205^{* * *} \\
(0.011)\end{array}$ & $\begin{array}{c}0.1208 * * * \\
(0.017)\end{array}$ & $\begin{array}{c}0.1152^{* * *} \\
(0.018)\end{array}$ & $\begin{array}{c}0.1211 * * * \\
(0.011)\end{array}$ & $\begin{array}{c}0.1171 * * * \\
(0.011)\end{array}$ & $\begin{array}{c}0.1176^{* * *} \\
(0.017)\end{array}$ & $\begin{array}{c}0.1124 * * * \\
(0.018)\end{array}$ & $\begin{array}{c}0.1201 * * * \\
(0.012)\end{array}$ & $\begin{array}{c}0.1160 * * * \\
(0.012)\end{array}$ & $\begin{array}{c}0.1166^{* * *} \\
(0.019)\end{array}$ & $\begin{array}{c}0.1110^{* * *} \\
(0.019)\end{array}$ & $\begin{array}{c}0.1197 * * * \\
(0.012)\end{array}$ & $\begin{array}{c}0.1159 * * * \\
(0.012)\end{array}$ & $\begin{array}{c}0.1167 * * * \\
(0.018)\end{array}$ & $\begin{array}{c}0.1115^{* * *} \\
(0.019)\end{array}$ \\
\hline Educ $\times$ College & & & $\begin{array}{l}0.0911 \\
(0.046)\end{array}$ & $\begin{array}{l}0.0893 \\
(0.046)\end{array}$ & & & $\begin{array}{l}0.0887 \\
(0.046)\end{array}$ & $\begin{array}{l}0.0871 \\
(0.046)\end{array}$ & & & $\begin{array}{l}0.0847 \\
(0.046)\end{array}$ & $\begin{array}{l}0.0835 \\
(0.046)\end{array}$ & & & $\begin{array}{l}0.0866 \\
(0.046)\end{array}$ & $\begin{array}{l}0.0854 \\
(0.046)\end{array}$ \\
\hline Income PGI & & & & & $\begin{array}{c}0.0290 * \\
(0.012)\end{array}$ & $\begin{array}{c}0.0278^{*} \\
(0.012)\end{array}$ & $\begin{array}{c}0.0285^{*} \\
(0.012)\end{array}$ & $\begin{array}{l}0.0274^{*} \\
(0.012)\end{array}$ & $\begin{array}{l}0.0786 \\
(0.066)\end{array}$ & $\begin{array}{l}0.0691 \\
(0.065)\end{array}$ & $\begin{array}{l}0.0752 \\
(0.067)\end{array}$ & $\begin{array}{l}0.0659 \\
(0.065)\end{array}$ & $\begin{array}{l}0.0430 \\
(0.036)\end{array}$ & $\begin{array}{l}0.0383 \\
(0.035)\end{array}$ & $\begin{array}{l}0.0410 \\
(0.036)\end{array}$ & $\begin{array}{l}0.0364 \\
(0.035)\end{array}$ \\
\hline $\begin{array}{l}\text { Parental Educ } \\
\mathrm{R}^{2}\end{array}$ & 0.249 & $\begin{array}{c}\mathrm{Y} \\
0.251\end{array}$ & 0.251 & $\begin{array}{c}\mathrm{Y} \\
0.253\end{array}$ & 0.252 & $\begin{array}{c}\mathrm{Y} \\
0.254\end{array}$ & 0.253 & $\begin{array}{c}\mathrm{Y} \\
0.255\end{array}$ & & $\mathrm{Y}$ & & $\mathrm{Y}$ & & $\mathrm{Y}$ & & $\mathrm{Y}$ \\
\hline Obs. & 6122 & - & - & - & - & - & - & - & - & - & - & - & - & - & - & - \\
\hline
\end{tabular}


Table A11. Average wages of major occupation groups in the UK (listed in order of highest wages)

\begin{tabular}{lc}
\hline \multicolumn{1}{c}{ Major occupation group } & $\begin{array}{c}\text { Weekly wage averaged } \\
\text { over 2006-2010 (£) }\end{array}$ \\
\hline 1. Managers and Senior Officials & 807.74 \\
2. Professional Occupations & 688.72 \\
3. Associate Professional and Technical Occupations & 526.3 \\
5. Skilled Trades Occupations & 451.32 \\
8. Process, Plant and Machine Operatives & 412.68 \\
4. Administrative and Secretarial Occupations & 322.38 \\
6. Personal Service Occupations & 246.9 \\
9. Elementary Occupations & 232.38 \\
7. Sales and Customer Service Occupations & 202.32 \\
\hline
\end{tabular}


Table A12. Associations of polygenic index for income (without MTAG) in UK Biobank sibling pairs

\begin{tabular}{|c|c|c|c|c|}
\hline & \multirow[b]{2}{*}{ OLS } & \multirow[b]{2}{*}{ OLS-FE } & \multicolumn{2}{|c|}{ Conditional on education } \\
\hline & & & OLS & OLS-FE \\
\hline \multicolumn{5}{|l|}{ Socioeconomic outcomes } \\
\hline log hourly wage & $0.0581 * * *$ & $0.0325 * * *$ & $0.0158^{* * *}$ & 0.0161 \\
\hline$(N=17,692 \mid 17,578)$ & $(0.003)$ & $(0.006)$ & $(0.002)$ & $(0.006)$ \\
\hline top household income & $0.0459 * * *$ & $0.0280 * *$ & $0.0167 * * *$ & 0.0181 \\
\hline$(N=27,412 \mid 27,296)$ & $(0.003)$ & $(0.007)$ & $(0.003)$ & $(0.007)$ \\
\hline log regional income & $0.0315^{* * *}$ & $0.0143 * * *$ & $0.0143 * * *$ & $0.0112 *$ \\
\hline$(N=31,692 \mid 31,266)$ & $(0.001)$ & $(0.003)$ & $(0.001)$ & $(0.003)$ \\
\hline neighborhood score & $1.1489 * * *$ & $0.7167 * *$ & $0.3561 * * *$ & 0.5556 \\
\hline$(N=29,166 \mid 28,778)$ & $(0.088)$ & $(0.196)$ & $(0.088)$ & $(0.198)$ \\
\hline years of education & $1.0508 * * *$ & $0.5683 * * *$ & & \\
\hline$(N=35,132 \mid \mathrm{NA})$ & $(0.027)$ & $(0.065)$ & & \\
\hline college degree & $0.0986 * * *$ & $0.0500 * * *$ & & \\
\hline$(N=35,132 \mid \mathrm{NA})$ & $(0.002)$ & $(0.006)$ & & \\
\hline \multicolumn{5}{|l|}{ health proxies } \\
\hline waist-to-hip ratio & $-0.0051 * * *$ & -0.0029 & $-0.0028 * * *$ & -0.0022 \\
\hline$(N=35,498 \mid 35,028)$ & $(0.000)$ & $(0.001)$ & $(0.000)$ & $(0.001)$ \\
\hline BMI & $-0.3844 * * *$ & -0.1559 & $-0.2051 * * *$ & -0.1132 \\
\hline$(N=35,432 \mid 34,968)$ & $(0.027)$ & $(0.062)$ & $(0.027)$ & $(0.062)$ \\
\hline blood pressure & $-0.6487 * * *$ & -0.2545 & $-0.4445 * * *$ & -0.1968 \\
\hline$(N=31,770 \mid 31,372)$ & $(0.078)$ & $(0.200)$ & $(0.081)$ & $(0.203)$ \\
\hline lung function & $0.0453^{* * *}$ & 0.0044 & $0.0168^{* *}$ & -0.0032 \\
\hline$(N=30,240 \mid 29,844)$ & $(0.005)$ & $(0.012)$ & $(0.005)$ & $(0.012)$ \\
\hline \multicolumn{5}{|l|}{ disease diagnoses } \\
\hline ever hospitalized & $-0.0141 * * *$ & -0.0066 & $-0.0072 * *$ & -0.0053 \\
\hline$(N=35,602 \mid 35,132)$ & $(0.002)$ & $(0.006)$ & $(0.002)$ & $(0.006)$ \\
\hline ever diagnosed with cancer & 0.0001 & 0.0028 & -0.0002 & 0.0017 \\
\hline$(N=35,602 \mid 35,132)$ & $(0.002)$ & $(0.006)$ & $(0.002)$ & $(0.006)$ \\
\hline infectious and parasitic diseases & $-0.0088 * * *$ & 0.0007 & -0.0045 & 0.0012 \\
\hline$(N=35,602 \mid 35,132)$ & $(0.002)$ & $(0.005)$ & $(0.002)$ & $(0.005)$ \\
\hline neoplasms & 0.0004 & 0.0024 & -0.0004 & 0.0014 \\
\hline$(N=35,602 \mid 35,132)$ & $(0.002)$ & $(0.006)$ & $(0.002)$ & $(0.006)$ \\
\hline diseases of blood organs and immune system & $-0.0088^{* * *}$ & -0.0007 & -0.0039 & 0.0004 \\
\hline$(N=35,602 \mid 35,132)$ & $(0.002)$ & $(0.006)$ & $(0.002)$ & $(0.006)$ \\
\hline
\end{tabular}


Table A12. Associations of polygenic index for income (without MTAG) in UK Biobank sibling pairs

\begin{tabular}{|c|c|c|c|c|}
\hline & \multirow[b]{2}{*}{ OLS } & \multirow[b]{2}{*}{ OLS-FE } & \multicolumn{2}{|c|}{ Conditional on education } \\
\hline & & & OLS & OLS-FE \\
\hline endocrine, nutritional, and metabolic diseases & $-0.0152 * * *$ & -0.0032 & -0.0048 & -0.0008 \\
\hline$(N=35,602 \mid 35,132)$ & $(0.002)$ & $(0.006)$ & $(0.002)$ & $(0.006)$ \\
\hline mental, behavioral, nervous system disorders & $-0.0189 * * *$ & -0.0042 & $-0.0105^{* * *}$ & -0.0023 \\
\hline$(N=35,602 \mid 35,132)$ & $(0.002)$ & $(0.006)$ & $(0.002)$ & $(0.006)$ \\
\hline diseases of the eye and adnexa & $-0.0059^{* *}$ & -0.0031 & -0.0036 & -0.0022 \\
\hline$(N=35,602 \mid 35,132)$ & $(0.002)$ & $(0.005)$ & $(0.002)$ & $(0.005)$ \\
\hline diseases of the circulatory system & $-0.0264 * * *$ & -0.0128 & $-0.0149 * * *$ & -0.0105 \\
\hline$(N=35,602 \mid 35,132)$ & $(0.002)$ & $(0.007)$ & $(0.003)$ & $(0.007)$ \\
\hline diseases of the respiratory system & $-0.0173^{* * *}$ & -0.0075 & $-0.0109^{* * *}$ & -0.0057 \\
\hline$(N=35,602 \mid 35,132)$ & $(0.002)$ & $(0.006)$ & $(0.002)$ & $(0.006)$ \\
\hline diseases of the digestive system & $-0.0246^{* * *}$ & -0.0076 & $-0.0133 * * *$ & -0.0053 \\
\hline$(N=35,602 \mid 35,132)$ & $(0.003)$ & $(0.007)$ & $(0.003)$ & $(0.007)$ \\
\hline diseases of the skin and subcutaneous tissue & $-0.0047 *$ & -0.0014 & -0.0011 & 0.0000 \\
\hline$(N=35,602 \mid 35,132)$ & $(0.002)$ & $(0.005)$ & $(0.002)$ & $(0.005)$ \\
\hline diseases of musculoskeletal system and connective tissue & $-0.0237 * * *$ & -0.0096 & $-0.0136 * * *$ & -0.0070 \\
\hline$(N=35,602 \mid 35,132)$ & $(0.002)$ & $(0.007)$ & $(0.003)$ & $(0.007)$ \\
\hline diseases of genitourinary system & $-0.0162 * * *$ & -0.0036 & $-0.0080^{*}$ & -0.0017 \\
\hline$(N=35,602 \mid 35,132)$ & $(0.002)$ & $(0.007)$ & $(0.002)$ & $(0.007)$ \\
\hline symptoms and signs not elsewhere classified & $-0.0252 * * *$ & -0.0116 & $-0.0151 * * *$ & -0.0107 \\
\hline$(N=35,602 \mid 35,132)$ & $(0.003)$ & $(0.007)$ & $(0.003)$ & $(0.007)$ \\
\hline injury, poisoning, and other consequences of external causes & $-0.0073 * *$ & -0.0027 & -0.0042 & -0.0028 \\
\hline$(N=35,602 \mid 35,132)$ & $(0.002)$ & $(0.006)$ & $(0.002)$ & $(0.006)$ \\
\hline external causes of morbidity and mortality & $-0.0082 * * *$ & -0.0031 & -0.0044 & -0.0032 \\
\hline$(N=35,602 \mid 35,132)$ & $(0.002)$ & $(0.006)$ & $(0.002)$ & $(0.006)$ \\
\hline other health conditions & $-0.0222 * * *$ & -0.0145 & $-0.0126 * * *$ & -0.0129 \\
\hline$(N=35,602 \mid 35,132)$ & $(0.003)$ & $(0.008)$ & $(0.003)$ & $(0.008)$ \\
\hline
\end{tabular}

Note: Significance at family-wise error rate $5 \%(*), 1 \%(* *), 0.1 \%(* * *)$, where multiple hypothesis testing is corrected using Holm's method

(Holm, 1979) for each set of analysis. Standard errors clustered by family are reported in parentheses. The table reports the coefficient estimates for the standardized PGI for income (non-augmented). For each outcome, the sample is restricted to sibling pairs for both of whom the outcome is observed. FE indicates the models with family fixed effects included. The second column set reports the results where education is controlled for by including dummies for each qualification. As covariates we include dummy variables for the year of birth, male, and being a younger sibling as well as the top 20 genetic PCs. For the economic outcomes we control for the age dummies instead of the year of birth and add dummies for the year of survey. For BMI and waist-to-hip ratio we also control for the age dummies instead but not for the year of survey. In every case we also include the interaction terms between the male dummy and the rest of covariates. 
Table A13. Associations of polygenic index for educational attainment in UK Biobank sibling pairs

\begin{tabular}{|c|c|c|c|c|}
\hline & \multirow[b]{2}{*}{ OLS } & \multirow[b]{2}{*}{ OLS-FE } & \multicolumn{2}{|c|}{ Conditional on education } \\
\hline & & & OLS & OLS-FE \\
\hline \multicolumn{5}{|l|}{ Socioeconomic outcomes } \\
\hline log hourly wage & $0.0717 * * *$ & $0.0443 * * *$ & $0.0174 * * *$ & $0.0235^{* *}$ \\
\hline$(N=17,692 \mid 17,578)$ & $(0.002)$ & $(0.007)$ & $(0.002)$ & $(0.006)$ \\
\hline top household income & $0.0547 * * *$ & $0.0339 * * *$ & $0.0171^{* * *}$ & $0.0203^{*}$ \\
\hline$(N=27,412 \mid 27,296)$ & $(0.003)$ & $(0.007)$ & $(0.003)$ & $(0.007)$ \\
\hline log regional income & $0.0397 * * *$ & $0.0132 * *$ & $0.0185^{* * *}$ & $0.0093 *$ \\
\hline$(N=31,692 \mid 31,266)$ & $(0.001)$ & $(0.003)$ & $(0.002)$ & $(0.003)$ \\
\hline neighborhood score & $1.5003^{* * *}$ & 0.5229 & $0.5085^{* * *}$ & 0.3163 \\
\hline$(N=29,166 \mid 28,778)$ & $(0.088)$ & $(0.200)$ & $(0.091)$ & $(0.203)$ \\
\hline years of education & $1.3486^{* * *}$ & $0.7235^{* * *}$ & & \\
\hline$(N=35,132 \mid \mathrm{NA})$ & $(0.026)$ & $(0.066)$ & & \\
\hline college degree & $0.1281 * * *$ & $0.0662 * * *$ & & \\
\hline$(N=35,132 \mid \mathrm{NA})$ & $(0.002)$ & $(0.006)$ & & \\
\hline \multicolumn{5}{|l|}{ health proxies } \\
\hline waist-to-hip ratio & $-0.0063 * * *$ & $-0.0038^{* *}$ & $-0.0033 * * *$ & $-0.0029^{*}$ \\
\hline$(N=35,498 \mid 35,028)$ & $(0.000)$ & $(0.001)$ & $(0.000)$ & $(0.001)$ \\
\hline BMI & $-0.5545 * * *$ & $-0.2903 * * *$ & $-0.3452 * * *$ & $-0.2425 * *$ \\
\hline$(N=35,432 \mid 34,968)$ & $(0.027)$ & $(0.063)$ & $(0.028)$ & $(0.064)$ \\
\hline blood pressure & $-0.8190^{* * *}$ & $-0.6570^{*}$ & $-0.5865 * * *$ & $-0.5989 *$ \\
\hline$(N=31,770 \mid 31,372)$ & $(0.078)$ & $(0.208)$ & $(0.083)$ & $(0.212)$ \\
\hline lung function & $0.0521 * * *$ & 0.0192 & $0.0156^{* *}$ & 0.0102 \\
\hline$(N=30,240 \mid 29,844)$ & $(0.005)$ & $(0.013)$ & $(0.005)$ & $(0.013)$ \\
\hline \multicolumn{5}{|l|}{ disease diagnoses } \\
\hline ever hospitalized & $-0.0206^{* * *}$ & -0.0112 & $-0.0129 * * *$ & -0.0102 \\
\hline$(N=35,602 \mid 35,132)$ & $(0.002)$ & $(0.006)$ & $(0.002)$ & $(0.006)$ \\
\hline ever diagnosed with cancer & -0.0007 & -0.0004 & $-0.0009 * *$ & -0.0011 \\
\hline$(N=35,602 \mid 35,132)$ & $(0.002)$ & $(0.006)$ & $(0.002)$ & $(0.006)$ \\
\hline infectious and parasitic diseases & $-0.0125^{* * *}$ & -0.0067 & $-0.0068 * * *$ & -0.0057 \\
\hline$(N=35,602 \mid 35,132)$ & $(0.002)$ & $(0.005)$ & $(0.002)$ & $(0.005)$ \\
\hline neoplasms & 0.0006 & -0.0001 & -0.0004 & -0.0006 \\
\hline$(N=35,602 \mid 35,132)$ & $(0.002)$ & $(0.006)$ & $(0.002)$ & $(0.006)$ \\
\hline diseases of blood organs and immune system & $-0.0118^{* * *}$ & 0.0001 & $-0.0062 * *$ & 0.0008 \\
\hline$(N=35,602 \mid 35,132)$ & $(0.002)$ & $(0.007)$ & $(0.002)$ & $(0.007)$ \\
\hline
\end{tabular}


Table A13. Associations of polygenic index for educational attainment in UK Biobank sibling pairs

\begin{tabular}{|c|c|c|c|c|}
\hline & \multirow[b]{2}{*}{ OLS } & \multirow[b]{2}{*}{ OLS-FE } & \multicolumn{2}{|c|}{ Conditional on education } \\
\hline & & & OLS & OLS-FE \\
\hline endocrine, nutritional, and metabolic diseases & $-0.0270 * * *$ & -0.0136 & $-0.0152 * * *$ & -0.0114 \\
\hline$(N=35,602 \mid 35,132)$ & $(0.002)$ & $(0.006)$ & $(0.002)$ & $(0.006)$ \\
\hline mental, behavioral, nervous system disorders & $-0.0275^{* * *}$ & -0.0084 & $-0.0174 * * *$ & -0.0055 \\
\hline$(N=35,602 \mid 35,132)$ & $(0.002)$ & $(0.006)$ & $(0.002)$ & $(0.006)$ \\
\hline diseases of the eye and adnexa & $-0.0060 * *$ & -0.0062 & $-0.0031 * *$ & -0.0053 \\
\hline$(N=35,602 \mid 35,132)$ & $(0.002)$ & $(0.005)$ & $(0.002)$ & $(0.005)$ \\
\hline diseases of the circulatory system & $-0.0348 * * *$ & -0.0119 & $-0.0211 * * *$ & -0.0098 \\
\hline$(N=35,602 \mid 35,132)$ & $(0.003)$ & $(0.007)$ & $(0.003)$ & $(0.007)$ \\
\hline diseases of the respiratory system & $-0.0209 * * *$ & -0.0079 & $-0.0131 * * *$ & -0.0062 \\
\hline$(N=35,602 \mid 35,132)$ & $(0.002)$ & $(0.006)$ & $(0.002)$ & $(0.006)$ \\
\hline diseases of the digestive system & $-0.0335 * * *$ & -0.0143 & $-0.0202 * * *$ & -0.0118 \\
\hline$(N=35,602 \mid 35,132)$ & $(0.003)$ & $(0.008)$ & $(0.003)$ & $(0.008)$ \\
\hline diseases of the skin and subcutaneous tissue & $-0.0082 * * *$ & -0.0040 & $-0.0048 * *$ & -0.0024 \\
\hline$(N=35,602 \mid 35,132)$ & $(0.002)$ & $(0.005)$ & $(0.002)$ & $(0.005)$ \\
\hline diseases of musculoskeletal system and connective tissue & $-0.0354 * * *$ & $-0.0256^{* *}$ & $-0.0241 * * *$ & $-0.0229 *$ \\
\hline$(N=35,602 \mid 35,132)$ & $(0.002)$ & $(0.007)$ & $(0.003)$ & $(0.007)$ \\
\hline diseases of genitourinary system & $-0.0220 * * *$ & -0.0111 & $-0.0128 * * *$ & -0.0099 \\
\hline$(N=35,602 \mid 35,132)$ & $(0.002)$ & $(0.007)$ & $(0.003)$ & $(0.007)$ \\
\hline symptoms and signs not elsewhere classified & $-0.0317 * * *$ & -0.0136 & $-0.0200 * * *$ & -0.0127 \\
\hline$(N=35,602 \mid 35,132)$ & $(0.003)$ & $(0.008)$ & $(0.003)$ & $(0.008)$ \\
\hline injury, poisoning, and other consequences of external causes & $-0.0088 * * *$ & -0.0035 & $-0.0051 * *$ & -0.0023 \\
\hline$(N=35,602 \mid 35,132)$ & $(0.002)$ & $(0.006)$ & $(0.002)$ & $(0.006)$ \\
\hline external causes of morbidity and mortality & $-0.0106^{* * *}$ & -0.0038 & $-0.0061^{* *}$ & -0.0028 \\
\hline$(N=35,602 \mid 35,132)$ & $(0.002)$ & $(0.006)$ & $(0.002)$ & $(0.006)$ \\
\hline other health conditions & $-0.0325 * * *$ & $-0.0243 *$ & $-0.0219 * * *$ & $-0.0224 *$ \\
\hline$(N=35,602 \mid 35,132)$ & $(0.003)$ & $(0.008)$ & $(0.003)$ & $(0.008)$ \\
\hline
\end{tabular}

Note: Significance at family-wise error rate $5 \%(*), 1 \%(* *), 0.1 \%(* * *)$, where multiple hypothesis testing is corrected using Holm's method (Holm, 1979) for each set of analysis. Standard errors clustered by family are reported in parentheses. The table reports the coefficient estimates for the standardized PGI for educational attainment. For each outcome, the sample is restricted to sibling pairs for both of whom the outcome is observed. FE indicates the models with family fixed effects included. The second column set reports the results where education is controlled for by including dummies for each qualification. As covariates we include dummy variables for the year of birth, male, and being a younger sibling as well as the top 20 genetic PCs. For the economic outcomes we control for the age dummies instead of the year of birth and add dummies for the year of survey. For BMI and waist-to-hip ratio we also control for the age dummies instead but not for the year of survey. In every case we also include the interaction terms between the male dummy and the rest of covariates. 


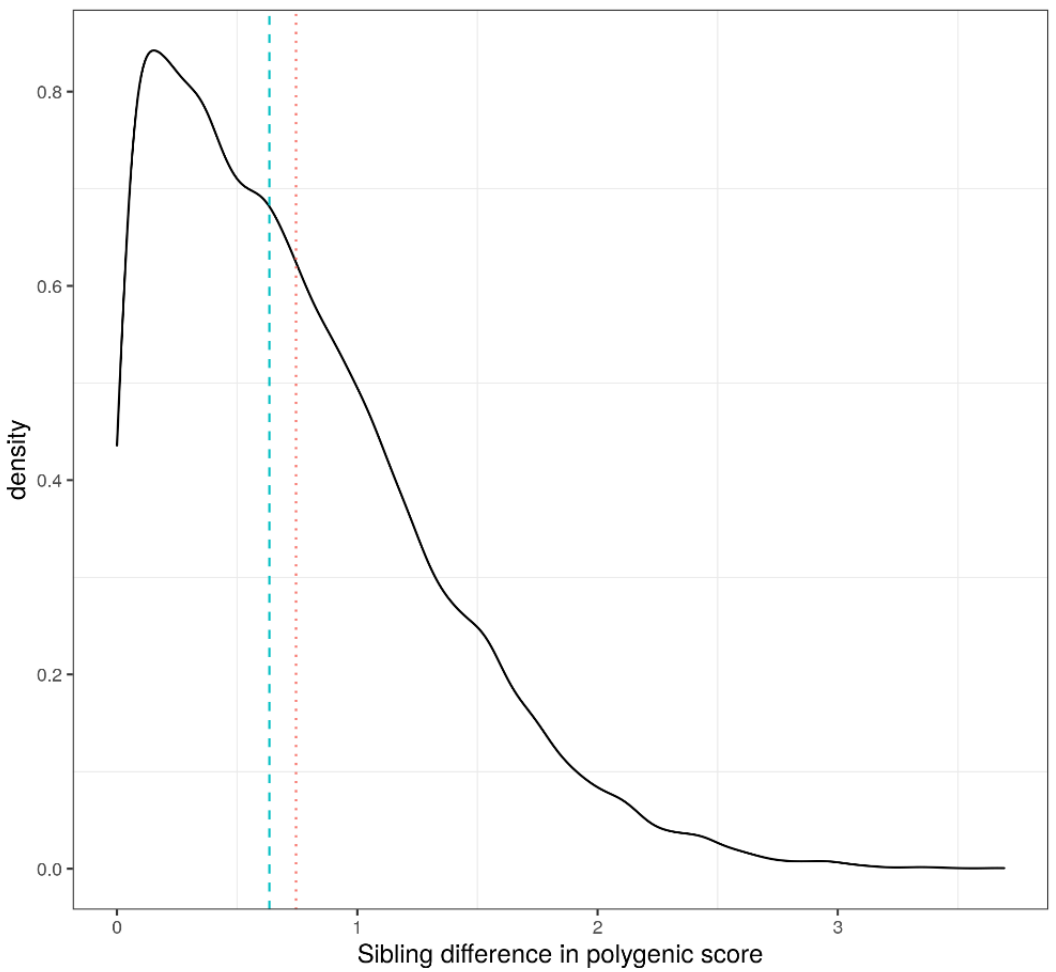

mean $=0.745 \quad$ median $=0.634$

Figure A1. Distribution of the differences in MTAG polygenic indices (PGI) for income between siblings in the UK Biobank

Note: The figure plots the density distribution of the absolute difference in the standardized PGI between siblings with Gaussian kernel. 
"* log occupational wage per hour -

"top household income

"* log regional income -

* college degree -

ever hospitalized -

ever diagnosed with cancer -

Infectlous and parastitic diseases -

neoplasms -

.

endocrine, nutritional, and metabolic diseases -

mental, behavioral, nervous system disorders -

diseases of the eye and adnexa -

diseases of the circulatory system -

diseases of the respiratory system -

diseases of the digestive system -

diseases of the skin and subcutaneous tissue -

-

diseases of genitourinary system -

symptoms and signs not elsewhere classified

Injury, poisoning, and other consequences of external causes -

external causes of morbidility and mortallity -

other health conditions -

$-0.05$

0.05

0.10

OLS

- OLS with

- OLS|edu

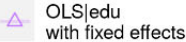

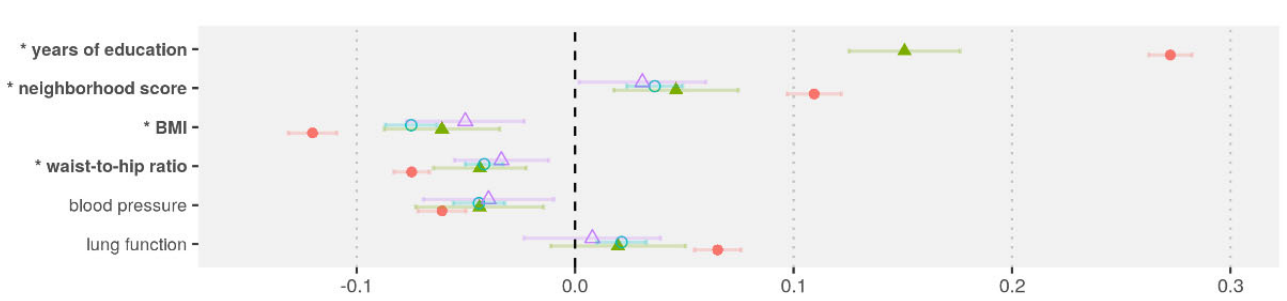

Figure A2. Associations of MTAG polygenic index (PGI) for income in UK Biobank sibling pairs (OLS)

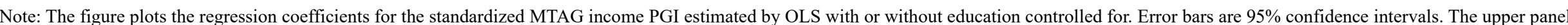

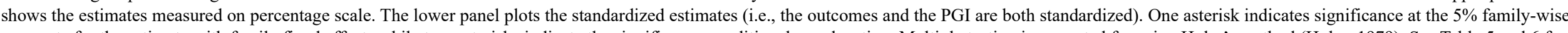

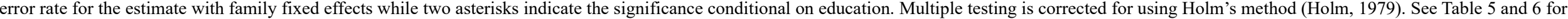
more details. 


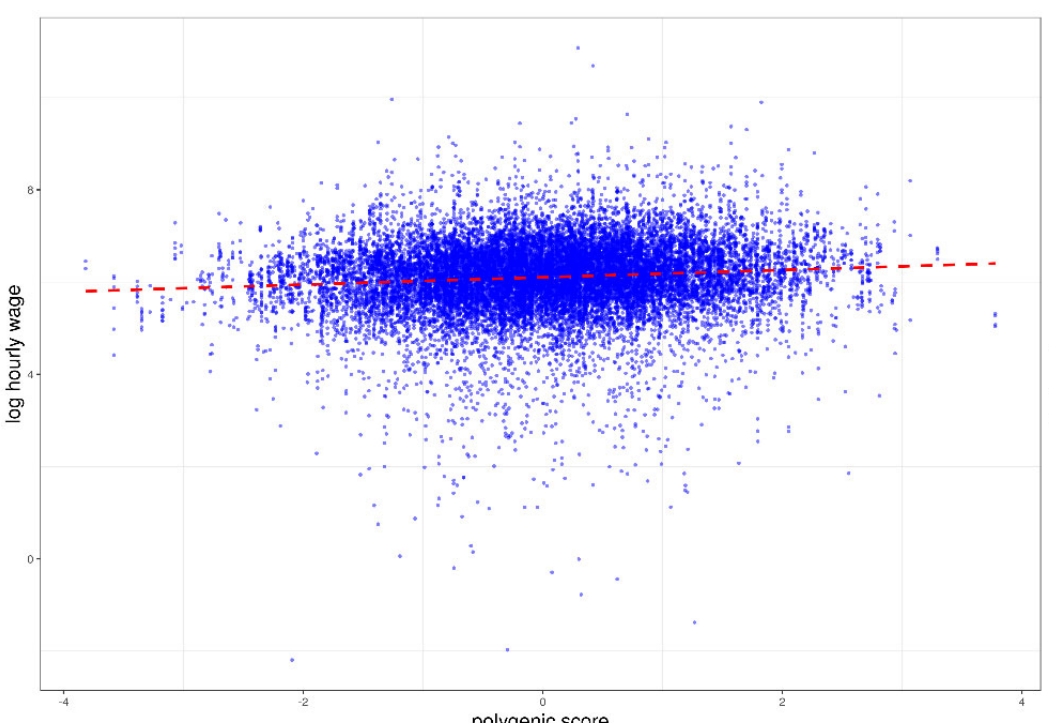

polygenic score

a. HRS

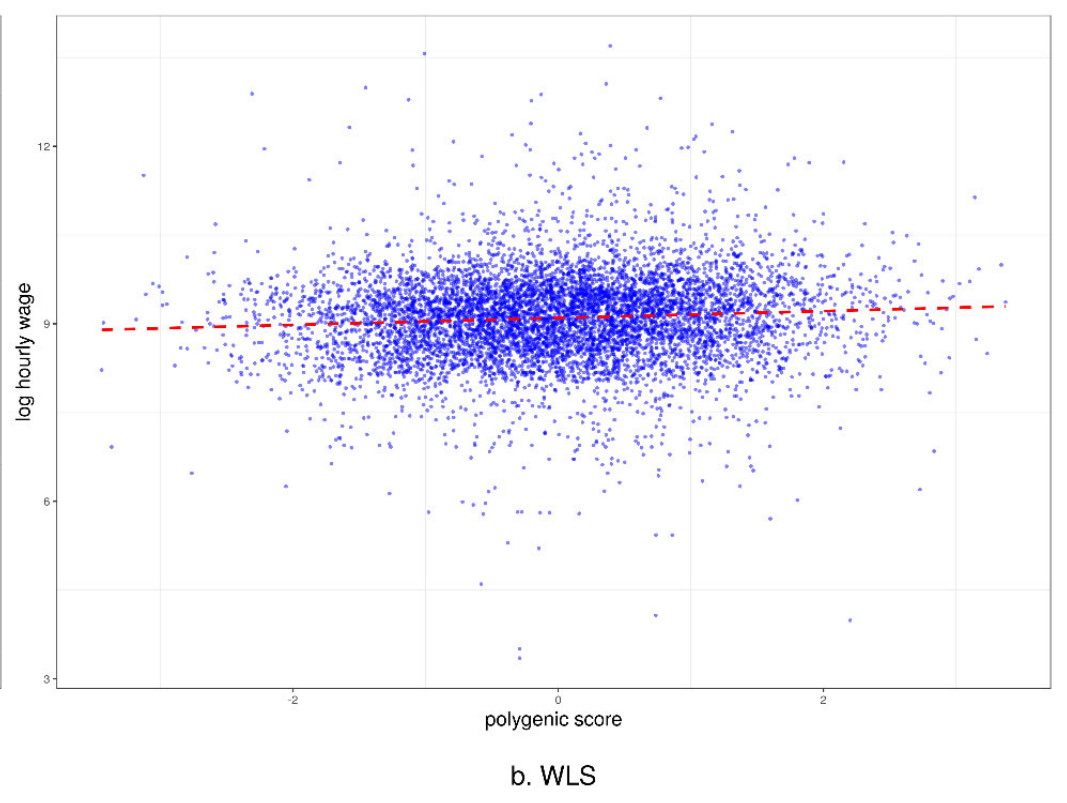

Figure A3. Scatter plots of log hourly wages and polygenic indices for income

Note: The left figure presents a scatter plot for the sample from the Health and Retirement Study $(N=22,247)$, while the right for the sample from the Wisconsin Longitudinal Study $(N=7,387)$. The dotted lines are regression lines. 


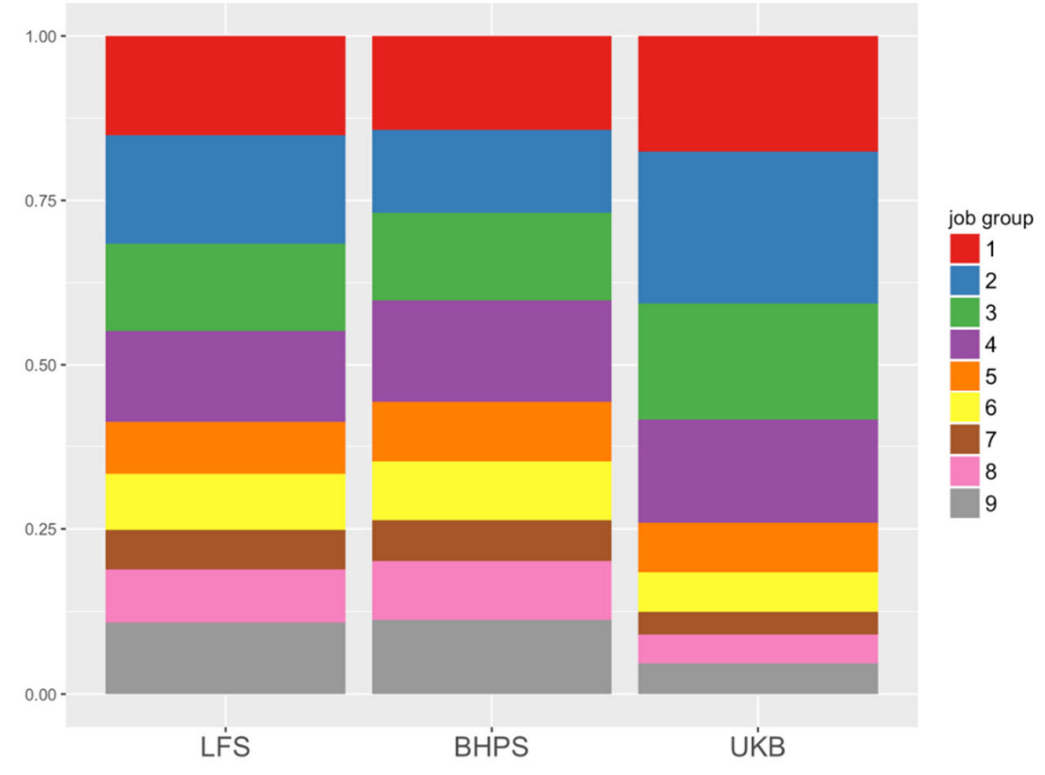

Figure A4. The composition of major occupation groups in the UK

Note: see Table A11 for the major occupation group reference. 

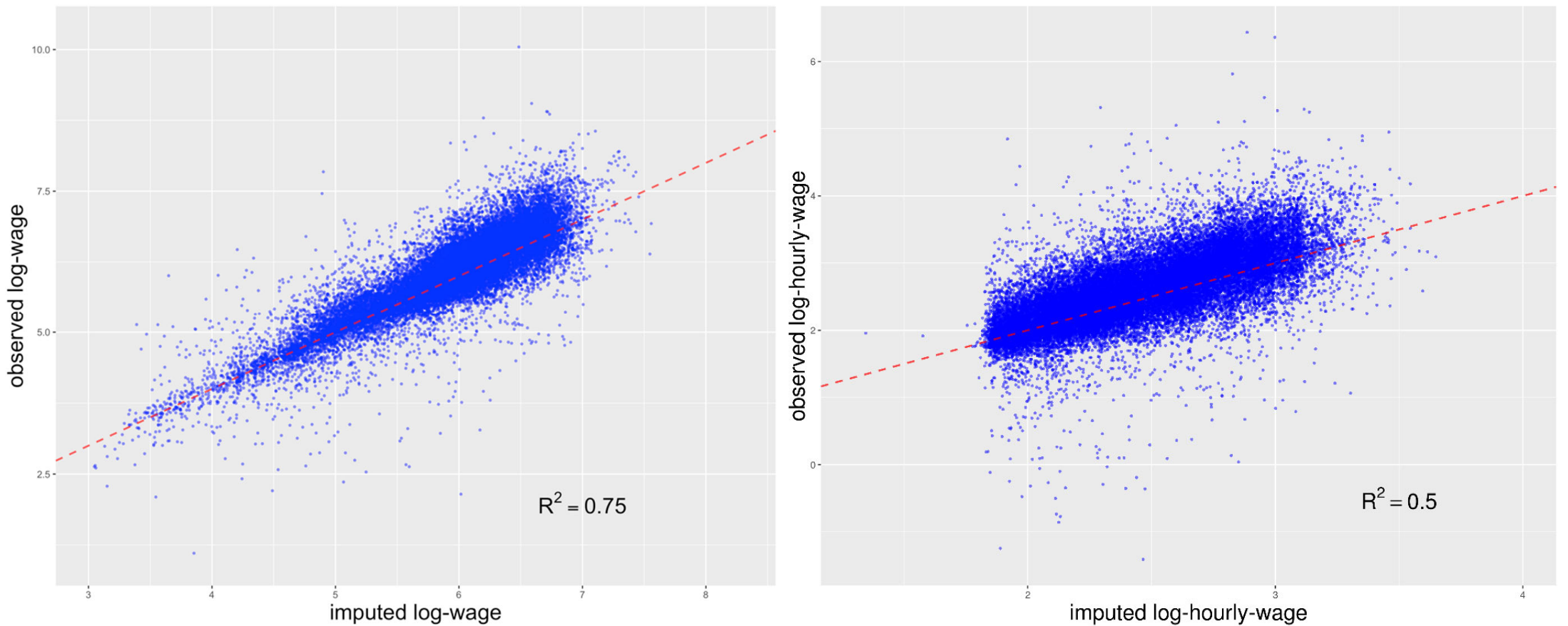

Figure A5. Scatter plots of imputed and observed log-wages in the BHPS $(N=32,947)$ 


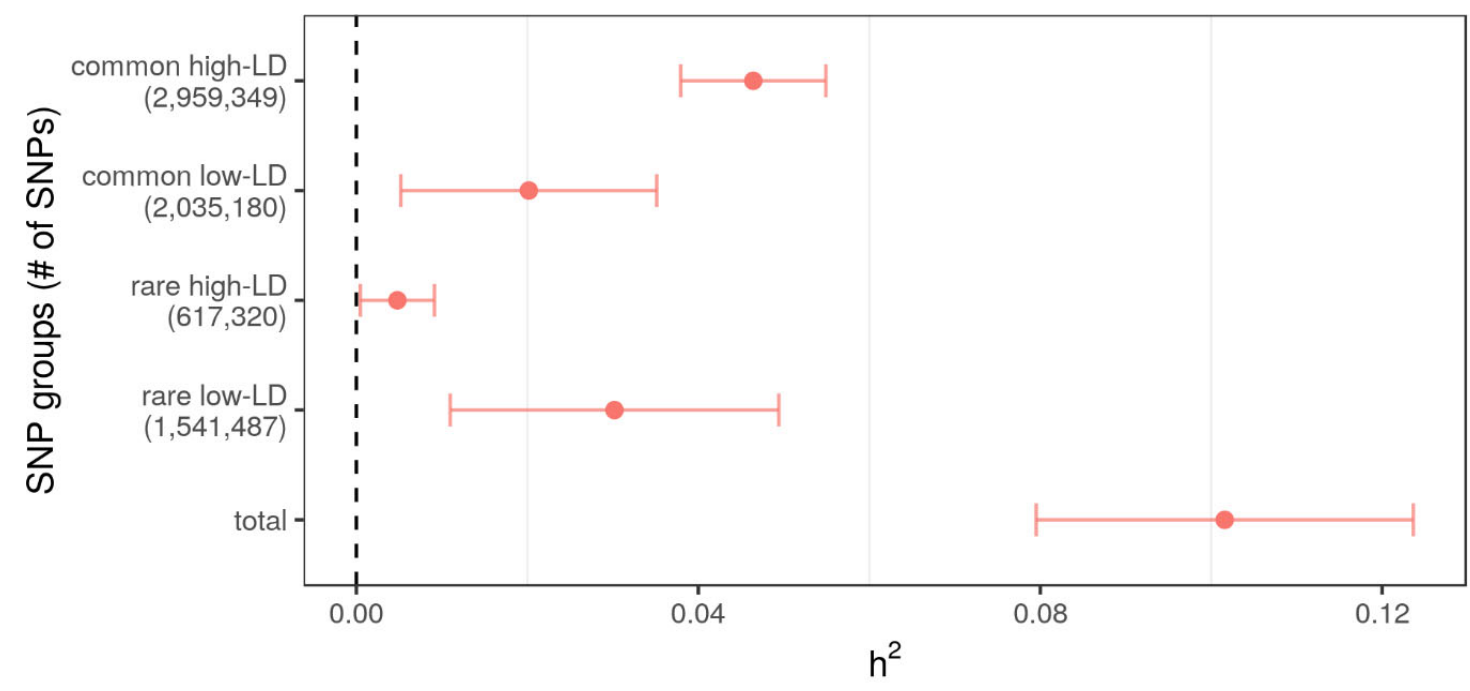

Figure A6. Decomposition of the total heritability of occupational wages by different groups of single nucleotide polymorphisms (SNPs)

Note: GREML estimation in 24,000 unrelated individuals from the UK Biobank. Error bars are standard errors. SNPs are sorted into four groups by different genetic features. LD - linkage disequilibrium scores. 


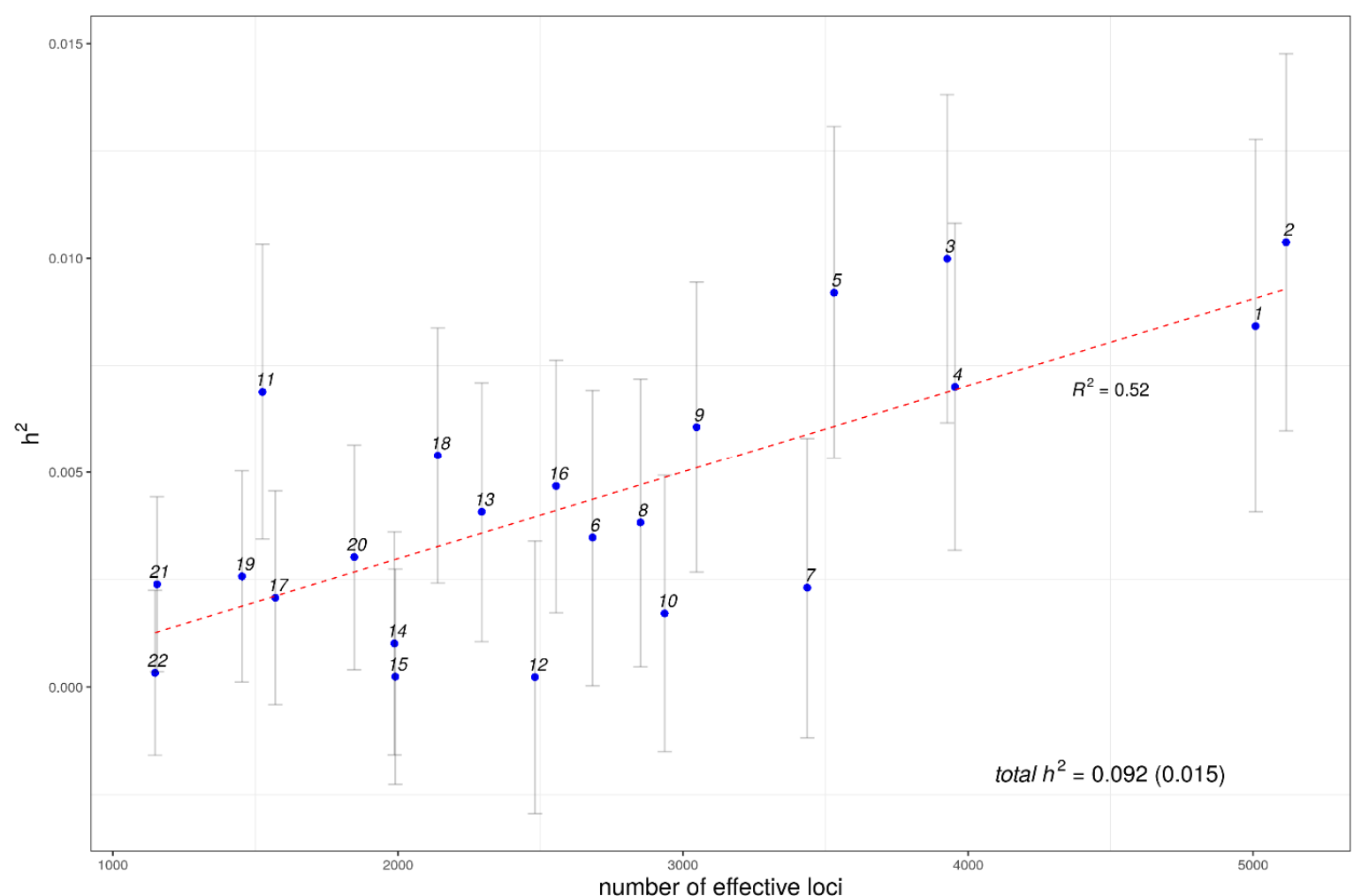

Figure A7. Decomposition of the total heritability of occupational wages by chromosomes

Note: GREML estimation in 24,000 unrelated individuals from the UK Biobank. Error bars are standard errors from GREML

estimation. The standardized coefficient of a regression of chromosomal $h^{2}$ SNP on the effective number of loci per chromosome is 0.72 (95\% CI: 0.41 - 1.02) 


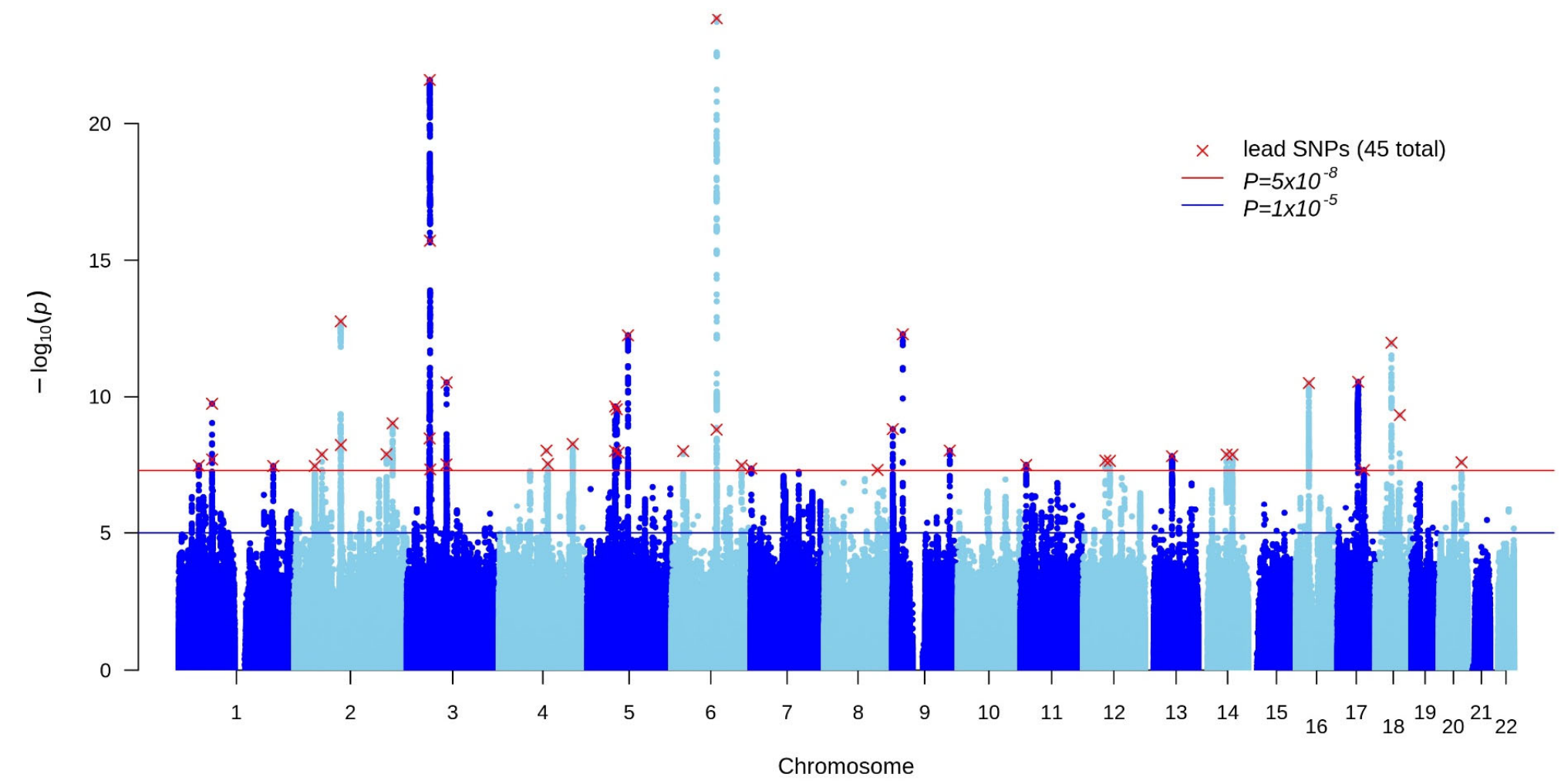

Figure A8. Manhattan plot for a genome-wide association study (GWAS) of occupational wages in the UK Biobank

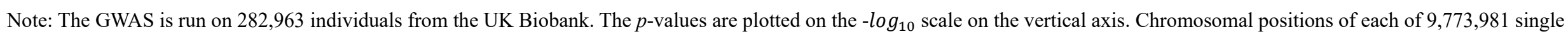
nucleotide polymorphisms (SNPs) are plotted on the horizontal axis. 


\section{Frequently Asked Questions (FAQs)}

This document provides information about the study:

Kweon et al. (2020) "Genetic Fortune: Winning or Losing Education, Income, and Health", a scientific article that was posted as a Tinbergen Institute Discussion Paper (https://papers.tinbergen.nl/20053.pdf). This research project was led by Dr. Philipp Koellinger (Vrije Universiteit Amsterdam \& University of Wisconsin-Madison). The paper has not yet been peer-reviewed. The paper and this FAQ are expected to change in response to feedback from the scholarly community, journalists, and members of the public.

This FAQ was prepared by several of the study's coauthors and draws from and builds on the FAQs for earlier SSGAC papers, as well as the FAQ of (Demange et al. 2020), which was written by one of the coauthors of the present study, Paige Harden. Questions and comments about the paper or this FAQ should be sent to Philipp Koellinger (koellinger@wisc.edu). 


\section{Table of contents:}

$\begin{array}{lr}\text { Summary } & 3\end{array}$

The current study $\quad 4$

Who conducted this study? $\quad 4$

$\begin{array}{ll}\text { What did you do in this paper? } & 4\end{array}$

$\begin{array}{ll}\text { How was income measured in the current study? } & 6\end{array}$

$\begin{array}{ll}\text { Did you find the gene(s) for income? } & 7\end{array}$

Are the genetic variants associated with income in your study also associated with other outcomes? 8

$\begin{array}{ll}\text { How good is your polygenic index? } & 8\end{array}$

What do you mean by "genetic fortune"? $\quad 9$

What does your study not mean? $\quad 9$

Is this the first GWAS on income? 10

Implications of the study $\quad 10$

Does this study show that an individual's level of income, education, or health is determined, or fixed, $\begin{array}{ll}\text { at conception? } & 10\end{array}$

Can your polygenic index be used to predict how well someone will do in life? 11

Can your polygenic index be used for research studies in non-European-ancestry populations? 12

What policy lessons do you draw from this study? How could society benefit from this work? 13

Could this kind of research lead to discrimination against, or stigmatization of, people with the relevant genetic variants? If so, why conduct this research? 14

$\begin{array}{ll}\text { Background } & 15\end{array}$

Why did you study income? What are the goals of the study? 15

What does it mean to say that income is "heritable"? 16

What is a GWAS? Are the genetic variants identified in a GWAS "causal”? 16

$\begin{array}{ll}\text { What is a polygenic index? } & 18\end{array}$

Where can I learn more about social science genetics? 19

$\begin{array}{ll}\text { Appendices } & 21\end{array}$

$\begin{array}{ll}\text { Quality control measures } & 21\end{array}$

$\begin{array}{ll}\text { Additional reading and references } & 22\end{array}$ 


\section{Summary}

- Our study investigates the importance of genetic fortune for inequalities in education, income, and health. The measure of genetic fortune that we use is an index that summarizes the effects of genetic markers that are associated with income. Our results show that genetic endowments and their implications contribute to inequality, but the implications of genetic endowments are malleable, for example via policies targeting education.

- The results of our study are a snapshot of currently existing social realities - they are not universal truth, "given by nature", or unchangeable.

- Social and other environmental factors are the main drivers of variation in income.

- To construct the polygenic index for income, we carried out the first genome-wide association study (GWAS) on individual income using a sample of $N=282,963$ individuals of European ancestries in the UK Biobank. We found 45 approximately independent genetic loci, each with a tiny effect size that captures less than $0.04 \%$ of the variance in income. The genetic index constructed from these GWAS results based on $\approx 1.5 \sim 2.5$ million measured genetic markers accounts for $\approx 1 \%$ of the variation in self-reported income in two independent samples in the US, and for up to $\approx 2.8 \%$ in a hold-out sample in the UK Biobank. Our analyses also estimate an upper bound for how precise this index could potentially be if much larger GWAS samples were available. This upper bound is approximately $10 \%$ of the variance in income.

- Analyses in a hold-out sample of $\approx 35,000$ siblings in the UK Biobank show that a sibling who was by chance endowed with the higher genetic index for income tends to have more favorable outcomes throughout life, including a higher chance of obtaining a college degree, of obtaining a well-paid job, of living in a good neighborhood, and of being healthy.

- We demonstrate that genetic fortune for income influences health outcomes partly via its effect on educational attainment, which depends on the existing environment and can be intervened upon e.g., with policy reforms. We also demonstrate that the ex post returns to schooling remain substantial even after controlling for genetic confounds, with an average of $8-11 \%$ higher hourly wages earned for each additional year of education in a US sample.

- Our study implies in no way whatsoever that some people are "naturally" superior to others or that social inequality is inevitable. We wholeheartedly reject such claims on both scientific and moral grounds. Instead, our study demonstrates that exogenously given genetic endowments matter for inequality, but the implications of these endowments depend on the environment and can be changed. For example, it is possible that some of the implications of genetic endowments that we find reflect currently existing discriminatory practices. Genetic associations and the results of our study do not justify discrimination in any way.

- Our genetic index and the results of our GWAS on income cannot be used

- to make meaningful comparisons across different groups (e.g., groups that differ in their ancestries, their self-perceived "race", or in the environment they live in) or

- to make meaningful predictions for any specific person. 


\section{The current study}

\subsection{Who conducted this study?}

This study was conceptualized and led by Philipp Koellinger and carried out by his research group (Hyeokmoon Kweon, Casper Burik, Richard Karlsson Linnér, Aysu Okbay, Ronald de Vlaming) at the Department of Economics at Vrije Universiteit Amsterdam, in collaboration with Thomas DiPrete (Columbia University, Sociology) and K. Paige Harden (University of Texas Austin, Psychology). Daphne Martschenko (Stanford University, Biomedical Ethics) brought an adversarial collaborative perspective to this paper and the FAQ, assisting with the socially-responsible communication of research findings, improving the accessibility of this study to multiple audiences, and keeping the historically-burdened legacies of genetics research at the forefront of our thinking on how best to frame our research.

\subsection{What did you do in this paper?}

Our study explores how important luck is for how well people do in life. This is an important question because inequalities in society that are due to luck are often perceived as unfair and less desirable than inequalities due to hard work and effort (Gromet, Hartson, and Sherman 2015; Cappelen et al. 2013; Almås et al. 2010; Alesina, Stantcheva, and Teso 2018; Alesina and Ferrara 2005; Clark and D'Ambrosio 2015). The two specific types of luck we consider in our study are who one's parents are and which parts of their genes they passed on. We call these two types of luck the social and the genetic lottery, respectively. The social lottery refers to the resources and the specific type of environments parents provide for their children. The genetic lottery refers to the specific version of the genes that parents pass on to their children, which is a random draw from the genes that someone's mother and father inherited from their respective parents. Both the social and the genetic lottery are luck in the sense that nobody has any sort of influence on who their parents are and which genes those parents passed on. However, both of these lotteries determine the starting points of children in life and can restrict the equality of opportunities among people. Our study investigates how important these two types of luck are for the type of education people achieve, what type of job they get, how much they earn, and how healthy they are. The results of our study are a snapshot of currently existing social realities - they are not universal truth, "given by nature", or unchangeable.

Genes may influence lifetime outcomes in various ways and a specific gene may have positive implications for one specific outcome, but no or even negative implications for another. Thus, to investigate the implications of the genetic lottery, we made a choice about which lifetime outcome to focus on. We decided to focus on the results of the genetic lottery for income because income is an important dimension of inequality that is of central importance for quality of life (also see the FAQ section Why did you study income?).

Our study proceeded in several steps. The first four steps were necessary preparatory analyses we needed to do before we could get to the core analyses we present in our study. We present those preparatory analyses in the online appendix of our study and only describe them briefly in the main text. 
First, we constructed an approximate measure of individual income for the largest sample in our study, the UK Biobank, from detailed occupational codes and information about typical incomes in these occupations in the UK from additional data sources (How was income measured in the current study?). This first step was necessary because the UK Biobank did not have any direct measure of individual income. We call the measure that we constructed "occupational wages".

Second, we estimated the heritability of occupational wages using the molecular genetic data that the UK Biobank had collected. More precisely, we looked at a sample of UK Biobank participants that was not closely related to each other and that all had White British ancestries (Quality control measures). In this subsample, we calculated the extent to which people were genetically similar. On average, a sample of unrelated individuals will have a genetic similarity of zero. However, any pair of individuals may have a genetic similarity that is slightly smaller or larger than zero by pure chance. We then use these "per chance" genetic similarities to estimate the extent to which they are related to observed similarities in occupational wages. Importantly, our estimates define the upper bound for how good a polygenic index for income based on currently measured genetic markers could potentially be (What is a polygenic index?). Our estimates of the "SNP-based" heritability of occupational wages (What does it mean to say that income is "heritable"?) suggest that approximately $10 \%$ of the variance in occupational wages is captured by the common genetic variants that we observed and used to calculate genetic similarities between people.

Third, we run a GWAS (What is a GWAS?) to estimate associations between over 1 million common SNPs and occupational wages in the UKB. This part of our analyses identified 45 approximately independent genetic loci for occupational wages, each with a tiny effect size. Specifically, each SNP we identified captures less than $0.04 \%$ of the variance in occupational wages.

As a fourth step, we take the estimates of the SNP effects in the UKB to construct polygenic indices for income in two population samples from the US, the Health and Retirement Study ( $\underline{H R S})$, and the Wisconsin Longitudinal Study (WLS)), that both contain genetic data and detailed information about individual income (What is a polygenic index?). It turns out that our polygenic index is associated with income in both of these samples, capturing approximately $1 \%$ of the observed variance in income. In other words, our GWAS succeeded in finding some genetic signal that has a bearing on the distribution of income in samples that were collected in a different country using different methods.

We then proceeded to the core questions of our study that are presented in the main text. First, we demonstrate in a sample of approximately 35,000 siblings in the UK Biobank sample that individuals with higher occupational wages or those having a college degree are much healthier in late adulthood than those with lower occupational wages or those without a college degree. However, if we only look at differences between the siblings using a statistical technique called family fixed effects, we see that the relationship between socioeconomic status and health is much weaker and in many cases indistinguishable from zero. Siblings share the same family environment and their genes are drawn from the same "pool" (the genes of their parents). Thus, our family fixed effect results show that the combined outcomes of the social and the genetic lottery account for a large share of the health-advantages of high socioeconomic status. 
Next, we look at the outcomes of the genetic lottery for income among the UK Biobank siblings. Our results show that genetic luck for income contributes to inequalities between siblings throughout the life course how long they go to school, what types of jobs they get, and how healthy they are in late adulthood. However, we also show that these health inequalities due to genetic luck for income are partially mediated by educational attainment. Since educational attainment depends partly on existing policies, labor market conditions, and many other environmental factors, this finding illustrates that the effects of the genetic lottery are not deterministic and can be changed.

Finally, we investigate whether the well-known positive relationship between high education and high income may be partly due to genetic confounds that were previously unobserved. Our results show that although genetic factors are still associated with income even after we take educational differences between people into account, the income growth with education remains positive and strong, with an average of 8$11 \%$ higher hourly wages for each additional year of education in the US sample we studied. Thus, far from suggesting any kind of biological determinism, our genetically informed study results point to important mechanisms that are driving inequality and that can be intervened upon (What does your study not mean?).

\subsection{How was income measured in the current study?}

Our GWAS used a measure of occupational wages, which we derived from standardized occupation codes and information about typical wages in those occupations. We verified our results with two samples that had self-reported measures of income.

More generally, the focus of the present study is on individual income, which is the most direct measure of the consumption and savings opportunities that a person has. Individual income is the result of various factors including achieved qualifications (e.g., education, learned occupation, experience), personal characteristics (e.g., leadership, cognitive skills, consciousness), the demand and supply for these qualifications and characteristics in the labor market, and personal choices about labor supply (e.g., due to personal preferences, and decisions about division of labor among household members).

That said, income can be measured in many different ways. For example, it can be measured at the household level or the individual level, and measures may include or exclude specific sources of income (e.g., government transfers, pensions, investment gains, and profits from business ownership).

However, most large datasets that contain genetic information do not have measures of individual income. This was also the case in the UK Biobank sample that we used for our GWAS and our sibling analyses. However, the UK Biobank did include detailed information about the type of job people have (or had before they retired). This information is available in standardized occupation codes. We used these codes to impute a proxy of individual income that we refer to as occupational wages. Using additional datasets that are representative of the UK population, we show that our occupational wage variable captures approximately $70 \%$ of the variance in weekly wages among individuals in the UK. We note that the occupational wage variable primarily captures differences in income between occupations and has little bearing on differences in income within occupations other than those that can be attributed to age and sex. 
To confirm that our GWAS (also see the FAQ section What is a GWAS? Are the genetic variants identified in a GWAS "causal"?) on occupational wages captures something "real", we tested the polygenic indices (What is a polygenic index?) derived from these GWAS results in two samples from another country (the US) that both had different, more detailed information about individual income. We found that our polygenic indices capture approximately $1 \%$ of the variance in these samples. This is not a lot, but it is a proof of principle that our GWAS results carry over to other samples and other measures of income, allowing us to use these results to obtain an estimate of the ex post returns to schooling that controlled for potential genetic confounds (What did you do in this paper?).

\subsection{Did you find the gene(s) for income?}

No.

We did not find "the gene for" income or anything else. We identified many genetic variants that are associated with income. Although it was once believed that scientists would discover numerous one-to-one associations between genes and outcomes, we have known for many years that the vast majority of human traits and other outcomes are complex and are influenced by many (thousands or even millions of) genes, each of which alone tends to have a small influence on the relevant outcome (Chabris et al. 2015).

Although we did find several genes that are associated with income, we believe that characterizing these as "genes for income" is likely to mislead, for many reasons.

First, a large part of the variation in people's income is accounted for by social and other environmental factors, not by additive genetic effects. "Genes for income" might be read to imply, incorrectly, that genes are the strongest predictor of variation in income - this is not the case.

Second, the genetic variants that are associated with income are also associated with many other things (only some of which we identify in this study, see, for example. our results on the links between income and health). These variants are no more "for" income than for the other outcomes with which they are associated (e.g., BMI, Alzheimer's disease, HDL cholesterol levels).

Third, each individual genetic variant captures only a tiny part of the variance in income (less than $0.04 \%$ ). Our results suggest that hundreds, or even thousands, of genetic variants are associated with income, but each of them considered by itself has only a tiny effect. The phrase "genes for income" might misleadingly imply large effects of specific genes, but these effects do not exist.

Fourth, environmental factors can increase or decrease the impact of specific genetic variants. Put differently, even if a genetic variant is associated with higher or lower levels of income on average, it may have a much larger or smaller effect depending on environmental conditions. For example, our results show a slightly higher heritability of income for females than for males, and higher returns to schooling for females. Although we do not explore the reasons for these results in our paper, they could be driven by the different environmental conditions that males and females are facing, including different gender role models, potential discrimination, and income differences across occupations that offer more opportunities for males to earn high wages despite having low educational attainment (Dougherty 2005). 
Finally, genes do not affect income directly. Rather, their influence works via environmental channels that are subject to change. For example, our results suggest that the associations between genes and income work partly through educational attainment which can be influenced by policy interventions.

\subsection{Are the genetic variants associated with income in your study also associated with other outcomes?}

Yes.

From the 45 SNPs that we report in our paper, 26 were previously found to be associated with average household income (Hill et al. 2019) and 31 loci with educational attainment (Lee et al. 2018). Thus, the genetic architectures of different measures of socioeconomic attainment are largely similar. Importantly, we also found that all except one of our 45 SNPs were previously found to be associated (Kevles 1995) with health outcomes such as BMI, HDL cholesterol levels, diabetes, bipolar disorder, alcohol consumption, Parkinson's disease and many others. Thus, genetic factors linked to income also tend to have relevance for health and vice versa. There is no clear line separating the genetics of health and income, which is not surprising given the well-known relationships between socioeconomic status and health (Wilkinson and Marmot 2003; Stringhini et al. 2017; Piotrowska et al. 2015; Chetty et al. 2016).

\subsection{How good is your polygenic index?}

Our polygenic index captures approximately $1 \%$ of the variance in income in two US samples and up to $3 \%$ of the variance in occupational wages in a hold-out sample from the UK Biobank. (see also the FAQ section What is a polygenic index?). This figure is good enough for important research purposes such as exploring the relationships between health and income (What did you do in this paper?) but is completely useless for (misguided) attempts to "predict" individual differences from genetic data (see the FAQ 1.5). Figure A1 in our paper illustrates this point.

Note that our estimates of the "SNP heritability" of income (Table 2) suggest an upper bound of $\sim 10 \%$ for the potential accuracy of polygenic indices for income in the future, as GWAS sample becomes larger (see the FAQ What is a GWAS?). Even a polygenic index of $\sim 10 \%$ would not nearly be accurate enough to make meaningful statistical predictions at the individual level - see Figure 3 in (Harden and Koellinger 2020). Furthermore, our sibling analyses (Table 5) suggest that approximately half of the signal our polygenic index picks up is actually due to environmental effects that are correlated with genes, such as the rearing environment provided by one's parents. Even the remaining effects are partially mediated by environmental channels such as educational attainment (Table 5). This finding clearly illustrates what we said here earlier - polygenic indices are not a clean way to separate biological and environmental influences. Genetic effects on socioeconomic outcomes such as income do not exist in a vacuum they are shaped by environmental conditions that keep evolving and that are not equal for everyone. 


\subsection{What do you mean by "genetic fortune"?}

Our lives are influenced by two major sources of luck that are completely outside of our control. The first one is who our parents are and what type of environments they provide for us (a "social lottery"). The second is which particular set of genes we inherited from our parents (a "genetic lottery"). We refer to the outcomes of the latter, the genetic lottery, as genetic fortune. In fact, the process of meiosis randomly mixes the genotypes of our biological parents, providing biological siblings with different outcomes of the genetic lottery. Our study exploits these random genetic differences between siblings for a variety of purposes, e.g., to better understand the links between income and health (Table 6).

\subsection{What does your study not mean?}

Genetic research has a long history of being misinterpreted and misused to argue that social inequality is inevitable, that social programs designed to improve people's lives are bound to fail and that some people are "naturally" inferior to other people (Kevles 1995). We wholeheartedly reject these claims on both scientific and moral grounds.

A high or low polygenic index should not be interpreted to mean that someone is destined or determined to show a particular characteristic. It is not a "fortune teller." It is not a pure measure of someone's genetic "endowment." It is a risk factor. By way of analogy, having high cholesterol makes it more likely that an individual will have a heart attack, but it does not determine that outcome - lots of people have high cholesterol but don't have a heart attack, and you can take steps to prevent a heart attack if you are at high risk. Similarly, a high polygenic index means that an individual has a slightly higher probability of obtaining a higher income, but that higher probability does not mean destiny!

Genetic associations with income do not mean that the environment does not make a difference. In fact, polygenic indices such as ours partly capture what most people would consider as environmental effects. If, for example, parents with particular genetic variants are more likely to live in a wealthy neighborhood with good schools, and if good schools make it more likely that children will get good jobs later in their lives, then this means that their children's polygenic indices for income will be correlated with the quality of schools they attended. Our sibling analyses show that roughly half of the signal our polygenic index for income picks up is due to such indirect genetic effects or other environmental influences that just happen to be correlated with genes.

Genetic associations with income do not mean that interventions or policy reforms designed to combat inequalities are bound to fail. For example, interventions such as the Perry Preschool Project have shown that access to high-quality education is an effective way to improve lifetime outcomes, including a higher likelihood of getting a good job that pays well.

The existence of genetic associations with income within a group of people does not tell us anything about whether there are average differences between racial or ethnic groups, or why such differences, if they are observed, occur. This is an important point, because racist and classist ideas about the allegedly 
"inferior" character of people of color and the poor have been used to justify eugenic policies (Moore 2018; Crouch 2020; Carlson 2009). Nothing about this study gives any sort of empirical support to these ideas.

\subsection{Is this the first GWAS on income?}

No.

Previous GWAS have looked at household income. These studies uncovered many interesting findings that help to illuminate the complex relationships between socioeconomic conditions and health (Hill et al. 2016, 2019). However, it is challenging to correctly interpret genetic associations with such aggregate measures correctly (e.g., what does it mean if individual genetic variants are associated with household or neighborhood measures of prosperity?). Our approach to examining individual differences in income reduces this complexity to some extent. Additionally, many potential applications of genetic data in the social sciences require a clear focus on individual differences. One of our applications, the estimate of the returns to schooling, is an example of this - our genetically informed estimation required GWAS results on individual income as an input.

\section{Implications of the study}

3.1. Does this study show that an individual's level of income, education, or health is determined, or fixed, at conception?

No.

Social and other environmental factors are the main drivers of variation in income. However, even if it were true that genetic factors accounted for all of the differences among individuals in income, it would still not follow that an individual's income is "determined" at conception. There are at least three reasons for this:

First, some (if not all) genetic effects may operate through environmental channels (Jencks 1980). Our study clearly illustrates the relevance of educational attainment for income, and education can be changed through environmental interventions (e.g., policy).

Second, even if the genetic associations with income operated entirely through nonenvironmental mechanisms that are difficult to modify (such as direct influences on the formation of neurons in the brain and the biochemical interactions among them), powerful environmental interventions could still change these genetic relationships. In a famous example suggested by the economist Arthur Goldberger, even if all variation in unaided eyesight were due to genes, there would still be enormous benefits from introducing eyeglasses (Goldberger 1979). Similarly, policies that guarantee a minimum wage or a basic income have incontrovertible relevance for living standards and the overall distribution of income. 


\subsection{Can your polygenic index be used to predict how well someone will do in life?}

No (see also the FAQ sections What is a polygenic index? and How good are your polygenic indices?).

The figure below (Figure A1 in the paper) illustrates the statistical reason why the polygenic index cannot be used to predict how well someone will do in life. It shows data from the Health and Retirement Study, one of our replication samples in our paper. The $x$-axis plots the values of the polygenic index for income among 22,247 individuals. The values of the index in that sample were standardized to have a mean of zero and a standard deviation of one. The $y$-axis plots values of $\log$ hourly wages. Each dot in the figure represents the combination of the polygenic index and income for one specific person in the sample. Despite the small, positive relationship between the index and income in the sample (indicated by the dotted red regression line), it can be seen clearly that the values of the index are not very informative about the income of any specific individual in the sample. In fact, a very broad range of income values is observed even for people who have very low or very high values of the polygenic index (e.g., those that are two standard deviations away from the mean).

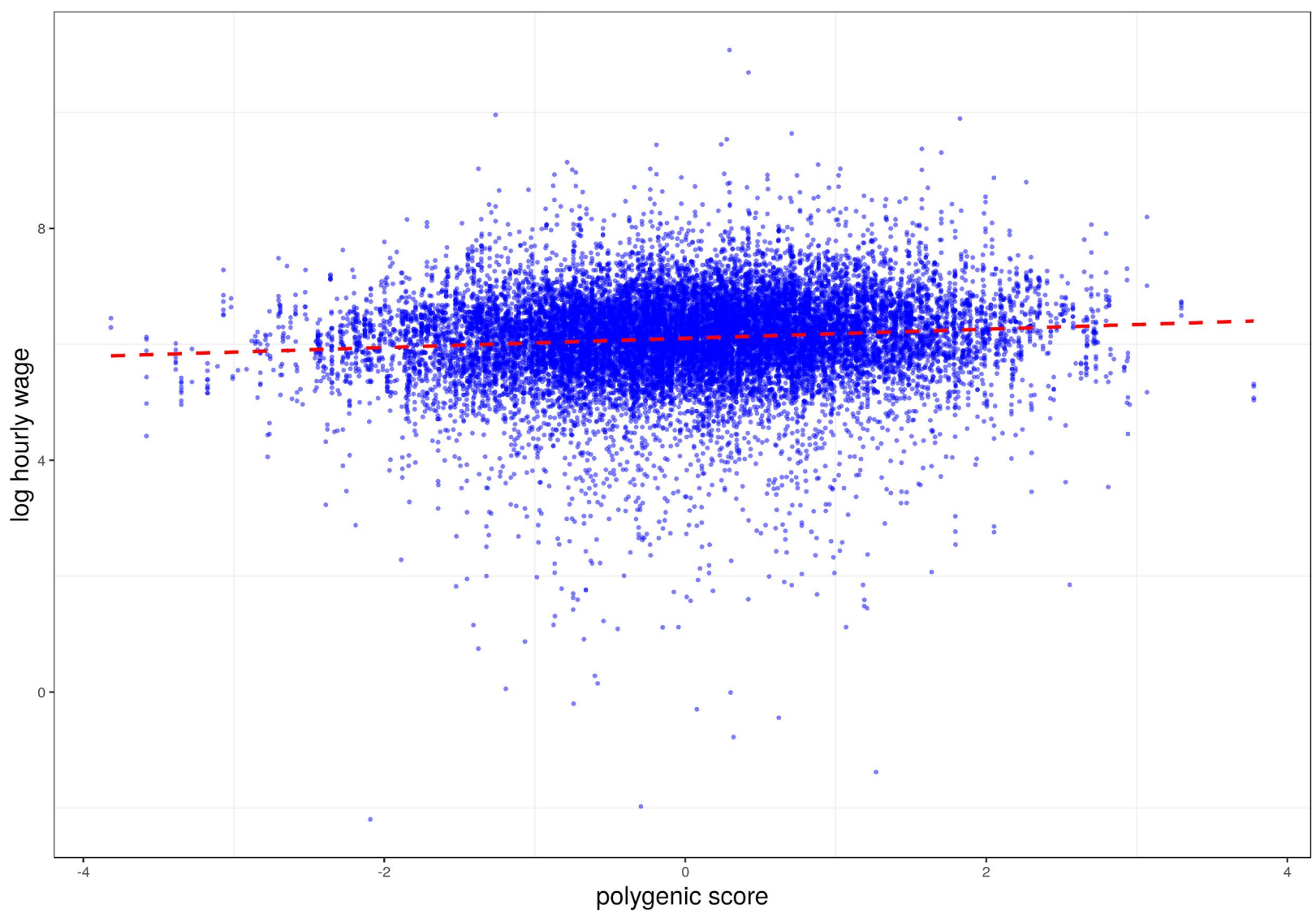

\section{a. HRS}

Note: The figure presents a scatter plot for the Health and Retirement Study $(N=22,247)$. The $\mathrm{x}$-axis shows standardized values of the polygenic index for occupational wages. The dotted line is a regression line with slope $0.083\left(p=1.04 \times 10^{-50}\right)$. 


\subsection{Can your polygenic index be used for research studies in non-European-ancestry populations?}

Only in a very limited way.

As a practical matter, it is possible to calculate a polygenic index for any individual for whom genomewide data are available, but the polygenic index can be expected to be much less accurate for non-Europeanancestry populations (Martin et al. 2019). For example, a polygenic index for educational attainment constructed from a GWAS in over one million individuals of white European ancestries captures 10.6\% of the variance in years of schooling among individuals with similar ancestries in the US Health and Retirement Study. The same index captures only $1.6 \%$ of the variance in years of schooling among AfricanAmericans in the same sample (Lee et al. 2018). For income, we expect a similarly strong attenuation of accuracy.

Our study was conducted only using samples of individuals of European ancestries (see the FAQ section Quality control measures). This choice was based primarily on data availability since the vast majority of all genotyped samples at the time of our study are of European ancestries (also see Footnote 9 in the manuscript). The set of SNPs that are associated with income in people of European ancestries is unlikely to overlap perfectly with the set of SNPs associated with EA in people of non-European ancestries. Even if a given SNP is associated in both ancestry groups, the effect size - in other words, the strength of the association-will almost surely differ, primarily because linkage disequilibrium (LD) patterns (i.e., the correlation structure of the genome) vary by ancestry (1000 Genomes Project Consortium et al. 2015). Thus, some variants may be associated with income because the variant is in LD (i.e., correlated) with a variant elsewhere in the genome that causally affects income (What is a GWAS? Are the genetic variants identified in a GWAS "causal"?). If the strength of the correlation is greater in one ancestry group than in another, then the size of the association will be larger in that ancestry group. Moreover, even if LD patterns were similar in each ancestry group, the association may differ in different groups because environmental conditions differ (What is a polygenic index?). The fact that there are differences across ancestry groups in the set of associated SNPs and their effect sizes has two important implications.

First, it means that the polygenic indices of individuals from different ancestry groups cannot be meaningfully compared. A recent paper (Martin et al. 2019) illustrated this point in the context of polygenic indices for predicting height; in the sample analyzed in that paper, polygenic indices for height for individuals of European ancestries are on average larger than those of South Asian ancestries which in turn are larger than those of African ancestries. In actuality, however, populations of African ancestries represented by the sample have similar heights to populations of European ancestries, and both African and European populations tend to be taller than South Asian populations.

Second, while polygenic indices may be used to study differences across individuals within a sample of people of non-European-ancestries, the accuracy of the index will be much smaller than that in a sample of people of European ancestries. Such an attenuation of predictive power has been repeatedly found in prior work (Domingue et al. 2015; Vassos et al. 2017; Domingue et al. 2017; Belsky et al. 2013). Unfortunately, this attenuation means that for non-European-ancestry populations, many of the benefits of having a polygenic index available will have to wait until large GWAS are conducted using samples from these 
populations. Currently, most large genotyped samples are of European ancestries, see (Mills and Rahal 2019). Footnote 9 in the paper discusses some reasons for this Eurocentric bias in the GWAS literature.

Third, the existence of genetic associations with income within a group of people (in our study, individuals of European ancestries) does not tell us anything about whether there are average differences between racial or ethnic groups, or why such differences, if they are observed, occur. This is an important point, because racist and classist ideas about the allegedly "inferior" character of people of color and the poor have been used to justify eugenic policies (Moore 2018; Crouch 2020; Carlson 2009). Nothing about this study gives any sort of empirical support to these ideas.

For a more extensive, excellent discussion of these and related issues, see Graham Coop's blog post "Polygenic scores and tea drinking": https://gcbias.org/2018/03/14/polygenic-scores-and-tea-drinking/.

\subsection{What policy lessons do you draw from this study? How could society benefit from this work?}

We do not make direct recommendations for policy in this paper.

However, our results clearly show that how well people do in life depends to a substantial degree on two lotteries - the social lottery (i.e., who your parents are) and the genetic lottery (i.e., which particular combination of your parents' genes you inherited). No one decides to participate in these lotteries or has any influence on their outcome. Our research demonstrates that both of these lotteries have far-reaching consequences across the lifespan, ranging from schooling, occupational choice, how much people can earn, what type of neighborhood they live in, all the way to how healthy people are in older age. This limits the extent to which people can take personal credit for the good things that happen to them and may inspire some humility. It also puts limits on how much people can be blamed for things that did not turn out in our favor. Furthermore, our results show that luck and agency (e.g., the choices people make, and how hard they work) cannot be clearly separated from one another - our polygenic index is associated with education and income, two measures that many may intuitively file under effort rather than luck. Our hope is that the insight that (genetic and social) luck matters may contribute to a greater sense of solidarity in society, more empathy for those who are unlucky, and a greater willingness to share among those who have the means to give. This is our preferred interpretation of our empirical results, which is in line with arguments put forward in moral philosophy (Rawls 1999; Roemer 1998) and with empirical studies that found that people are less willing to tolerate inequality that is due to luck rather than effort (Gromet, Hartson, and Sherman 2015; Cappelen et al. 2013; Almås et al. 2010; Cappelen, Sørensen, and Tungodden 2010; Alesina and Ferrara 2005; Alesina, Stantcheva, and Teso 2018). However, this is not the only way to interpret our results and different policy implications may be derived from different philosophical perspectives (Nozick 1974).

In general, the widespread availability of genetic data and the increasing insights into how genetic factors are associated with observed differences between people creates new challenges for policy making across various domains, including insurance markets, labor markets, personalized medicine, and reproductive technologies. In many of these areas, societies and policy makers will have to make difficult and complex trade-offs involving the protection of human rights including respect for autonomy, dignity, privacy, the right to science, the right to know or not to know about genetic results, as well as the feasibility of certain 
forms of insurance or possible improvements in health and well-being (Joly et al. 2020). One of the authors of the current study, Koellinger, recently wrote a policy report for the European Commission that reviews some of these challenges for policy making. This report can be accessed here.

We would like to state explicitly and emphatically that the genetic association results reported in the current study should not be used for any form of individual selection or discrimination (e.g., in labor or insurance markets or in reproductive technologies); see the FAQ sections What does your study not mean?, Can your polygenic index be used to predict how well someone will do in life?, and Can your polygenic index be used for research studies in non-European-ancestry populations? .

\subsection{Could this kind of research lead to discrimination against, or stigmatization of, people with the relevant genetic variants? If so, why conduct this research?}

Unfortunately, like a great deal of research - including, for instance, research identifying genomic variants associated with increased cancer risk - the results can be misunderstood and misapplied. This includes misuses that would discriminate against people who carry specific genetic variants (e.g., in insurance or labor markets). Nevertheless, for a variety of reasons, we do not believe that the best response to the possibility that useful knowledge might be misused is to refrain from producing that knowledge. Furthermore, we do not claim that genetically-informed studies are better than other empirical approaches. However, we briefly discuss some of the broad potential benefits of this research. We then describe what we take to be our ethical obligation as researchers conducting this work.

First, a benefit of conducting social science genetics research in ever larger samples is that doing so allows us to correct the scientific record. An important theme in earlier work by Koellinger and his colleagues in the SSGAC has been to point out that most existing studies in social-science genetics that report genetic associations with behavioral traits have serious methodological limitations, fail to replicate and are likely to be false-positive findings (Benjamin et al. 2012; van der Loos et al. 2011; Beauchamp et al. 2011; Karlsson Linnér et al. 2019). This same point was made in an editorial in Behavior Genetics (the leading journal for the genetics of behavioral traits), which stated that "it now seems likely that many of the published [behavior genetics] findings of the last decade are wrong or misleading and have not contributed to real advances in knowledge" (Hewitt 2012). One of the most important reasons why earlier work has generated unreliable results is that the sample sizes were far too small, given that the true effects of individual genetic variants on behavioral traits are small.

Second, social science genetics also has the potential to correct the social record and thereby to help combat discrimination and stigmatization. Our study clearly demonstrates that (i) the vast majority of income inequality is due to environmental factors, and (ii) even the genetic influences we identified are not deterministic. Instead, they also depend on environmental factors and work via environmental channels that can be influenced (e.g., via educational attainment).

Third, social science genetics research has the potential to yield many other benefits, as briefly summarized in the FAQ section Where can I learn more about social science genetics? and further explained in (Harden and Koellinger 2020). Foregoing this research necessarily entails foregoing these and any other possible benefits, some of which will likely be the result of serendipity rather than being foreseeable. 
In sum, we agree with the U.K. Nuffield Council on Bioethics, which concluded in a report (Bioethics 2002), p.114) that "research in behavioural genetics has the potential to advance our understanding of human behaviour and that the research can therefore be justified," but that "researchers and those who report research have a duty to communicate findings in a responsible manner." In our view, responsible behavioral genetics research includes a sound methodology and analysis of data; a commitment to publish all results, including any negative results; and the transparent, complete reporting of the methodology and findings in publications, presentations, and communications with the media and the public, including particular vigilance regarding what the results do-and do not-show (hence, this FAQ document).

\section{Background}

\subsection{Why did you study income? What are the goals of the study?}

Income has obvious relevance for the type of lives people can afford to live and our physical and mental well-being. Conversely, low income, poverty and economic deprivation are major risk factors for mental and physical diseases, lower life expectancy, and antisocial behavior (Wilkinson and Marmot 2003; Stringhini et al. 2017; Piotrowska et al. 2015). Furthermore, income is important for health and longevity not only for those in poverty, but also across the entire income distribution (Chetty et al. 2016). Thus, understanding the structural sources of inequalities in income is of fundamental importance both as a matter of both science and social policy (Piketty 1995).

It has long been recognized that parental income and socioeconomic status are major determinants of a child's expected trajectory in terms of cognitive and noncognitive skill development, behaviors, educational attainment, career prospects, and adult income. In other words, differences in income are partially transmitted across generations. At the same time, education, income, personality, cognitive abilities, and occupational choices are all heritable to some extent and parents pass on both their environments and their genes to their offspring. Thus, a major challenge in understanding the causes of inequality and social mobility is disentangling behavioral and environmental effects from possible genetic confounds and studying how they are related to each other.

One of the purposes of this study was to generate a set of publicly available genome-wide association study (GWAS) results on individual income that will provide researchers from various disciplines with new, better ways to study the causes and consequences of inequality and social mobility. We share the summary statistics of our GWAS with the research community at https://osf.io/rg8sh/, which allows others to construct a polygenic indix that will capture a part of the heritable component of individual differences in income. With this new data, researchers will be able to use this index to study a variety of important questions that will be informative about the structural sources of inequality.

We provide two examples of this in our study. First, we investigate the relationship between income and health outcomes in late adulthood, exploiting the random differences between siblings in their genetic propensity for high income as a "natural experiment". Specifically, we use the outcomes of the genetic lottery for income to gain new insights into the relationship between income and health outcomes later in 
life (see also the FAQ sections What did you do in this paper? and What do you mean by "genetic fortune"?). Second, we investigate the returns to schooling in a study design that allows us to control for potential genetic confounds that were unobserved until now. Our results illustrate the extent to which disparities among people in income and health are due to differences in genetic fortune and that choices about education influence income and health even after accounting for genetic effects.

\subsection{What does it mean to say that income is "heritable"?}

"Heritable" is a confusing word that is often misinterpreted to mean that heritable characteristics are determined by biology. This is not true. As we describe in our article in depth, heritable characteristics including income - can be influenced by the environment, can be changed by interventions or policy reforms, and are not "destined" to develop in a particular way based just on a person's genes. "Heritable" means that, within the sample of people being studied, those who are more genetically different from each other tend to show more different characteristics (Visscher, Hill, and Wray 2008). We return to this point - that heritable characteristics are not biologically determined - in the FAQ sections What is a GWAS? Are the genetic variants identified in a GWAS "causal"? and What does your study not mean?.

Previous studies have measured income and similar traits in identical and fraternal twins, and found that identical twins are more similar in their income skills than fraternal twins are (Taubman 1976). This result tells us that income is heritable. Using different data and a different approach (i.e., by quantifying whether observed genetic differences between individuals correspond to differences in income), we come to the same conclusion in our study. Because we now know that virtually all aspects of behavior and personality that differ between people are at least partly heritable, this result is not surprising (Turkheimer 2000; Polderman et al. 2015). The heritability of income should not be taken to mean that income is especially "genetic" in some way.

\subsection{What is a GWAS? Are the genetic variants identified in a GWAS "causal"?}

The short answers to these questions are as follows, with links to more details listed below:

- GWAS (genome-wide association studies) systematically scan the entire human genome for associations with a trait of interest, one SNP at a time.

- Correlations between SNPs are ignored.

- Not all potentially relevant genetic variants are included in a GWAS, partly because they may not have been measured precisely enough or measured at all.

- How frequently a particular genetic variant occurs in a population varies across places, which implies that genes and environments are correlated.

- GWAS try to control for potential correlations between genes and environments, but most GWAS (including ours) do so only imperfectly.

- Genes often influence outcomes only indirectly via environmental and behavioral pathways that can be changed.

- For all of the reasons above, genetic variants identified in a GWAS are often not causal. 
In a genome-wide association study (GWAS), scientists look at genetic variants measured across the entire human genome to see whether any of them are, on average, associated with higher or lower levels of some outcome. Similar to other studies our analyses focus on the most common genetic variants - so called single-nucleotide polymorphisms (SNPs). SNPs are sites in the genome where single DNA base pairs commonly differ across individuals. SNPs usually have two different possible base pairs, or alleles. Although there are tens of millions of sites where SNPs are located in the human genome, GWASs typically investigate only SNPs that can be measured (or imputed) with a high level of accuracy. Currently, such procedures usually yield millions of SNPs that together capture the most common genetic variations across people.

GWAS has been a successful research strategy for identifying genetic variants associated with many traits and diseases, including body height (Wood et al. 2014; Locke et al. 2015; Yengo et al. 2018), Alzheimer's disease (Jansen et al. 2018; Lambert et al. 2013), and schizophrenia (Ripke et al. 2014; Pardiñas et al. 2018). It has also recently been used to identify genetic variants associated with a variety of health-relevant social science outcomes, such as the number of children a person has (Barban et al. 2016), happiness (Okbay et al. 2016; Turley et al. 2018), and educational attainment (Rietveld et al. 2013; Okbay et al. 2016; Lee et al. 2018).

Statistical geneticists would call a genetic variant causal if a ceteris paribus change in that variant at conception would lead to a different outcome (e.g., higher income). Note that this definition of causality requires no minimum effect size (i.e., the change in income due to the change in the genetic variant may so small that it is barely measurable), nor does it require an understanding of the mechanism (i.e., the genetic effect may be indirect and mediated by environmental responses, self-selection into environments and so forth).

GWAS identifies genetic variants that are associated with the outcome, but an observed association with a specific variant does not imply that the variant causes the outcome, for a variety of reasons. First, genetic variants are often highly correlated with other, nearby variants on the same chromosome. As a result, when one or more variants in a region causally influence an outcome (in that particular environment), many noncausal variants in that region may also be identified as associated with the outcome. When GWAS results are analyzed, researchers often emphasize results for the genetic variant in a region that showed the strongest evidence of association. This variant does not need to be the causal variant. In fact, the causal genetic variant may not have even been measured directly. For example, GWAS that focus on common SNPs would not be able to identify rare or structural genetic variants (e.g., deletions or insertions of an entire genetic region) that are causal, but they may identify SNPs that are correlated with these unobserved variants.

Second, the frequencies of many genetic variants vary systematically across environments. If those environmental factors are not accounted for in the association analyses, some of the associations found may be spurious. To use a well-known example (Lander and Schork 1994), any genetic variants common in people of Asian ancestries will be associated statistically with chopstick use, but these variants would not cause chopstick use; rather, these genetic variants and the outcome of chopstick use are both distributed unevenly among people with different ancestries. This is the problem of "population stratification". GWAS 
researchers have a number of strategies for addressing the challenges posed by population stratification (see the FAQ section Quality control measures).

Even in studies such as ours that attempt to address and correct for heterogeneity in genetic ancestry, allele frequencies may nonetheless vary systematically with environmental factors. For example, a genetic variant that is associated with higher income in the parental generation may have downstream effects on children's educational outcomes (e.g., through living in a neighborhood with good schools). This same genetic variant is likely to be inherited by the children of these parents, creating a correlation between the presence of the genetic variant in a child's genome and the extent to which the child was reared in an environment with specific characteristics. A recent study of Icelandic families showed that the parental allele that is not passed on to the parent's offspring is still associated with the child's educational attainment, suggesting that GWAS results for educational attainment partly represent these intergenerational pathways (Kong et al. 2018). Our sibling analyses yield results that are consistent with this conclusion.

Third, variants' effects on an outcome may be indirect, so a variant that may be "causal" in one environment may have a diminished effect or no effect at all in other environments. For example, the nicotinic acetylcholine receptor gene cluster on chromosome 15 is associated with lung cancer (Thorgeirsson et al. 2008; Amos et al. 2008; Hung et al. 2008). From this observation alone we cannot conclude that these genetic variants cause lung cancer through some direct biological mechanism. In fact, it is likely that these genetic variants increase lung cancer risk through their effects on smoking behavior. In a tobacco-free environment, it is plausible that many of these associations would be substantially weaker and perhaps disappear altogether. Thus, even if we have credible evidence that a specific association is not spurious, it is entirely possible that the genetic variant in question influences the outcome through channels that we, in common parlance, would label environmental (e.g., smoking). Nearly forty years ago, the sociologist Christopher Jencks criticized the widespread tendency to mistakenly treat environmental and genetic sources of variation as mutually exclusive, see also (Turkheimer 2000). As the example of smoking illustrates, it is often overly simplistic to assume that "genetic explanations of behavior are likely to be exclusively physical explanations while environmental explanations are likely to be social" (Jencks 1980).

In general, GWAS is just one step in a longer, often complex process of identifying causal pathways, but the results of a large-scale GWAS are a useful tool for that purpose and often lead to novel and important insights (Visscher et al. 2017; Harden and Koellinger 2020). For example, a researcher may be interested in understanding the causal effect of some trait that develops and changes over the life course (e.g., socioeconomic status) on some outcome of interest (e.g., health). GWAS results and polygenic indices can be used as noisy, exogenously given proxies for this trait of interest. As a demonstration of this idea, our study uses random differences between siblings in the polygenic index for income to show that (i) higher socioeconomic status causes better health and (ii) that this effect partially works via educational attainment and its accompaniments (What did you do in this paper?).

\subsection{What is a polygenic index?}

The results of GWAS can be used to create a "polygenic index" (often also called a polygenic score), an index composed of many genetic variants from across the genome. More precisely, GWAS results are used to create a formula for how to construct a polygenic index. Using this formula, a polygenic index can then 
be constructed for any individual with genome-wide data. Indeed, some of the value of GWAS is that the polygenic index it produces can be used in subsequent studies conducted in other samples.

Because a polygenic index aggregates the information from many genetic variants, it is more strongly associated with variation among individuals for the GWAS outcome than with any single genetic variant. Often, the polygenic indices that are most strongly associated with an outcome are those created using all common genetic variants (typically more than a million) studied in GWAS. The larger the GWAS sample is, the more precise the polygenic index constructed from the GWAS results will be. If GWAS samples were infinitely large, the polygenic indices constructed from those samples would be expected to capture as much of the variance of a trait in an independent sample as implied by the SNP heritability of a trait. For example, in our study, we estimated the SNP heritability for income to be approximately $10 \%$. That means that a polygenic index constructed from an infinitely large GWAS on income would be expected to capture approximately $10 \%$ of the variance in income in an independent sample that was not included in the GWAS (Daetwyler, Villanueva, and Woolliams 2008; de Vlaming et al. 2017).

Note that this does not mean that $10 \%$ of anyone's income is biologically determined (see the FAQ sections What does your study not mean? and Does this study show that an individual's level of income, education, or health is determined, or fixed, at conception?). It is important to understand that polygenic indices are not a "clean" way to separate biological from nonbiological factors that contribute to differences between people's outcomes (Harden and Koellinger 2020). GWAS results are not entirely immune to unobserved (e.g., environmental) confounds, such as parenting, or neighborhood characteristics, and genetic influences are often conditional on and/or mediated by environmental channels (What is a GWAS? Are the genetic variants identified in a GWAS "causal"? and (Young et al. 2019)). For example, a society that systematically discriminates against people of color would induce a correlation between all genes for skin or hair pigmentation and income. However, a change in that society that would eliminate such discrimination could make these genetic associations disappear. Since polygenic indices merely aggregate the effects that were estimated in a GWAS, they partly reflect currently existing social realities. Furthermore, polygenic indices may exhibit different predictive accuracy even among members of the same ancestry group that vary from each other in terms of sex or socioeconomic status (Mostafavi et al. 2020).

\subsection{Where can I learn more about social science genetics?}

Two of the coauthors of the current study, Harden and Koellinger, recently published a review on social science genetics in Nature Human Behavior (Harden and Koellinger 2020) that provides a comprehensive answer. In short, we believe that the social sciences are incomplete without genetics because most differences between people in terms of their behavior, preferences, achievements, and life events are at least to some extent "heritable" (see the FAQ section What does it mean to say that income is "heritable"?). Thus, integrating molecular genetics into the social sciences presents an opportunity to deliver richer, more precise answers to old questions in psychology, sociology, economics and related fields. Furthermore, the new type of data and study designs that molecular genetics allow will grant social scientists opportunities to ask new questions and to pursue new answers that would not have previously been feasible. We see our current study as an example for both of these claims (What did you do in this paper?). 
The review by Harden and Koellinger also discusses the challenges of this work more broadly and provides several substantive examples of new insights afforded by integrating molecular genetic data into the social sciences (e.g., with respect to the intergenerational transmission of human capital, social mobility over the lifespan, human demography, gene-environment interactions, and the intersection between "social/behavioral" genetics and "disease" genetics). 


\section{Appendices}

\subsection{Quality control measures}

There are many potential pitfalls that could lead to spurious results in genome-wide association studies (GWAS). We took many precautions to guard against these pitfalls.

One potential source of spurious results is incomplete "quality control (QC)" of the genetic data. To avoid this problem, we used state-of-the-art QC protocols from medical genetics research (Winkler et al. 2014). We supplemented these protocols by developing and applying additional, more stringent QC filters.

Another potential source of spurious results is a confound known as "population stratification." To give a well-known illustration, suppose we were conducting a GWAS on the use of chopsticks (Lander and Schork 1994). People of Asian ancestries are far more likely to use chopsticks than people of European ancestries. If we combined samples of Chinese and European ancestries and performed a GWAS that ignores ancestry, then we would find genetic associations for these variants. However, those associations would simply reflect the fact that allele frequencies vary across ancestry groups.

In our study we were extremely careful to correct for population stratification as much as possible. At the outset, we restricted the study to individuals of European ancestries. As is standard in GWAS, we also controlled for "principal components" of the genetic data in the analysis; these principal components capture the small genetic differences across ancestry groups within European populations, so controlling for them largely removes the spurious associations arising solely from these small differences.

After taking these steps to minimize bias stemming from population stratification, we conducted $\square$ follow up analysis with $\sim 20,000$ pairs of siblings. These "within-family" analyses break the link between genes and the family environment, thanks to the natural experiment of meiosis. During meiosis, the two copies of each parental chromosome are randomly combined and then separated to create a set of two gametes (e.g., two eggs or two sperm) each of which contains only one new, resampled copy of each chromosome. This process creates an almost infinite number of different DNA sequences that each parent could theoretically pass on to their children. The resulting genetic differences between full siblings and dizygotic twins are therefore random and independent from environmental factors that vary between families. Therefore, comparisons among biological siblings yield estimates for our polygenic index that are immune to genetic nurture, an uncontrolled population structure in GWAS, or other sorts of environmental influences that cannot be traced back to direct genetic effects. In these analyses, we found that approximately half of the signal of our polygenic index is due to such environmental confounds, while the other half is due to genetic effects that originate in people we studied. We call these latter effects causal, even though the pathways from genes to outcomes are complex and not fully understood. 


\subsection{Additional reading and references}

Note: Whenever possible, we included links to freely available versions of these references.

1000 Genomes Project Consortium, Adam Auton, Lisa D. Brooks, Richard M. Durbin, Erik P. Garrison, Hyun Min Kang, Jan O. Korbel, et al. 2015. "A Global Reference for Human Genetic Variation." Nature 526 (7571): 68-74. https://europepmc.org/article/pmc/pmc4750478

Alesina, Alberto, and Eliana La Ferrara. 2005. "Preferences for Redistribution in the Land of Opportunities." Journal of Public Economics 89 (5-6): 897-931. http://darp.lse.ac.uk/papersDB/Alesina-LaFerrara_(JPubE05).pdf

Alesina, Alberto, Stefanie Stantcheva, and Edoardo Teso. 2018. "Intergenerational Mobility and Preferences for Redistribution." The American Economic Review 108 (2): 521-54. https://oconnell.fas.harvard.edu/files/alesina/files/alesina_stantcheva_teso_mobility_june_1.pdf

Almås, Ingvild, Alexander W. Cappelen, Erik Ø. Sørensen, and Bertil Tungodden. 2010. "Fairness and the Development of Inequality Acceptance." Science 328 (5982): 1176-78.

https://core.ac.uk/reader/52120866

Amos, Christopher I., Xifeng Wu, Peter Broderick, Ivan P. Gorlov, Jian Gu, Timothy Eisen, Qiong Dong, et al. 2008. "Genome-Wide Association Scan of Tag SNPs Identifies a Susceptibility Locus for Lung Cancer at 15q25.1." Nature Genetics 40 (5): 616-22.

https://europepmc.org/article/pmc/pmc2713680

Barban, Nicola, Rick Jansen, Ronald de Vlaming, Ahmad Vaez, Jornt J. Mandemakers, Felix C. Tropf, Xia Shen, et al. 2016. "Genome-Wide Analysis Identifies 12 Loci Influencing Human Reproductive Behavior.” Nature Genetics 48 (12): 1462-72. https://europepmc.org/article/pmc/pmc5695684

Beauchamp, Jonathan P., David Cesarini, Magnus Johannesson, Matthijs J. H. M. van der Loos, Philipp D. Koellinger, Patrick J. F. Groenen, James H. Fowler, J. Niels Rosenquist, A. Roy Thurik, and Nicholas A. Christakis. 2011. "Molecular Genetics and Economics." The Journal of Economic Perspectives: A Journal of the American Economic Association 25 (4): 57-82. http://fowler.ucsd.edu/molecular_genetics_and_economics.pdf

Belsky, Daniel W., Terrie E. Moffitt, Karen Sugden, Benjamin Williams, Renate Houts, Jeanette McCarthy, and Avshalom Caspi. 2013. "Development and Evaluation of a Genetic Risk Score for Obesity." Biodemography and Social Biology 59 (1): 85-100. https://europepmc.org/article/pmc/pmc3671353

Benjamin, Daniel J., David Cesarini, Matthijs J. H. M. van der Loos, Christopher T. Dawes, Philipp D. Koellinger, Patrik K. E. Magnusson, Christopher F. Chabris, et al. 2012. "The Genetic Architecture of Economic and Political Preferences." Proceedings of the National Academy of Sciences of the United States of America 109 (21): 8026-31. www.pnas.org/cgi/doi/10.1073/pnas.1120666109

Bioethics, Nuffield Council on. 2002. Genetics and Human Behavior: The Ethical Context. London: Nuffield Council on Bioethics.

Cappelen, Alexander W., James Konow, Erik Ø. Sørensen, and Bertil Tungodden. 2013. "Just Luck: An Experimental Study of Risk-Taking and Fairness." The American Economic Review 103 (4): 13981413. https://www.chapman.edu/research/institutes-and-centers/economic-scienceinstitute/_files/ifree-papers-and-photos/JamesKonow.pdf

Cappelen, Alexander W., Erik Ø. Sørensen, and Bertil Tungodden. 2010. "Responsibility for What? Fairness and Individual Responsibility.” European Economic Review 54 (3): 429-41. https://core.ac.uk/download/pdf/52121410.pdf

Carlson, Elof Axel. 2009. “Three Generations, No Imbeciles: Eugenics, the Supreme Court, and Buck v. Bell. By Paul A. Lombardo. Baltimore (Maryland): Johns Hopkins University Press. \$29.95. Xv 365 P.; Ill.; Index. 978-0-8018-9010-9. 2008." The Quarterly Review of Biology. 
https://doi.org/10.1086/603452.

Chabris, Christopher F., James J. Lee, David Cesarini, Daniel J. Benjamin, and David I. Laibson. 2015. "The Fourth Law of Behavior Genetics." Current Directions in Psychological Science 24 (4): 30412. https://scholar.harvard.edu/files/laibson/files/fourthlawbehaviorgenetics-accepted.pdf

Chetty, Raj, Michael Stepner, Sarah Abraham, Shelby Lin, Benjamin Scuderi, Nicholas Turner, Augustin Bergeron, and David Cutler. 2016. "The Association between Income and Life Expectancy in the United States, 2001-2014." JAMA: The Journal of the American Medical Association 315 (16): 1750.http://static.stevereads.com/papers_to_read/the_association_between_income_and_life_expect ancy_in the united_states_2001-2014.pdf

Clark, Andrew E., and Conchita D'Ambrosio. 2015. "Chapter 13 - Attitudes to Income Inequality: Experimental and Survey Evidence." In Handbook of Income Distribution, edited by Anthony B. Atkinson and François Bourguignon, 2:1147-1208. Elsevier.

Crouch, Caroline. 2020. "Molly Ladd-Taylor: Fixing the Poor: Eugenic Sterilization and Child Welfare in the Twentieth Century." Springer.

https://idp.springer.com/authorize/casa?redirect_uri=https://ink.springer.com/article/10.1007/s10964 -020-01218-w\&casa_token $=$ vQPCN-

yroYoAAAAA:iuJEb2oPmHxKeorxPy591LCGUtrAXqo1 kmyB9LYUgXDhht4O3163bqZieggTcM 1Ec_R-TVKRk5X8U2w.

Daetwyler, H. D., B. Villanueva, and J. A. Woolliams. 2008. "Accuracy of Predicting the Genetic Risk of Disease Using a Genome-Wide Approach.” PloS One 3 (10): e3395. https://journals.plos.org/plosone/article?id=10.1371/journal.pone.0003395

Demange, Perline A., Margherita Malanchini, Travis T. Mallard, Pietro Biroli, Simon R. Cox, Andrew D. Grotzinger, Elliot M. Tucker-Drob, et al. 2020. "Investigating the Genetic Architecture of NonCognitive Skills Using GWAS-by-Subtraction.” bioRxiv. https://doi.org/10.1101/2020.01.14.905794.

Domingue, Benjamin W., Daniel Belsky, Dalton Conley, Kathleen Mullan Harris, and Jason D. Boardman. 2015. "Polygenic Influence on Educational Attainment: New Evidence from The National Longitudinal Study of Adolescent to Adult Health." AERA Open 1 (3): 1-13. https://journals.sagepub.com/doi/full/10.1177/2332858415599972

Domingue, Benjamin W., Daniel W. Belsky, Amal Harrati, Dalton Conley, David R. Weir, and Jason D. Boardman. 2017. "Mortality Selection in a Genetic Sample and Implications for Association Studies." International Journal of Epidemiology. https://doi.org/10.1093/ije/dyx041.

Dougherty, Christopher. 2005. "Why Are the Returns to Schooling Higher for Women than for Men?" Journal of Human Resources XL (4): 969-88.

Goldberger, Arthur S. 1979. "Heritability." Economica 46 (184): 327-47. https://www-jstor-org.vunl.idm.oclc.org/stable/pdf/2553675.pdf?refreqid=excelsior\%3Ae5a9ce0528ad25c5c9a925fdbfd0bc9 $\mathrm{b}$

Gromet, Dena M., Kimberly A. Hartson, and David K. Sherman. 2015. "The Politics of Luck: Political Ideology and the Perceived Relationship between Luck and Success." Journal of Experimental Social Psychology 59 (July): 40-46.

https://labs.psych.ucsb.edu/sherman/david/sites/labs.psych.ucsb.edu.sherman.david/files/pubs/grome t_hartson_sherman_2015.pdf

Harden, K. P., and P. D. Koellinger. 2020. "Using Genetics for Social Science." Nature Human Behaviour. https://doi.org/10.1038/s41562-020-0862-5.

Hewitt, John K. 2012. "Editorial Policy on Candidate Gene Association and Candidate Gene-byEnvironment Interaction Studies of Complex Traits." Behavior Genetics 42 (1): 1-2. https://www.gwern.net/docs/genetics/heritable/2012-hewitt.pdf

Hill, W. David, Neil M. Davies, Stuart J. Ritchie, Nathan G. Skene, Julien Bryois, Steven Bell, Emanuele Di Angelantonio, et al. 2019. "Genome-Wide Analysis Identifies Molecular Systems and 149 Genetic Loci Associated with Income." Nature Communications 10 (1): 5741. https://www.nature.com/articles/s41467-019-135855?fbclid=IwAR0uwCWVJ3CCWbJ6qNiRyxHjguf93MNsh9bsqE6PALuB9AZByNxmIR4Wdys 
Hill, W. David, Saskia P. Hagenaars, Riccardo E. Marioni, Sarah E. Harris, David C. M. Liewald, Gail Davies, Aysu Okbay, Andrew M. McIntosh, Catharine R. Gale, and Ian J. Deary. 2016. "Molecular Genetic Contributions to Social Deprivation and Household Income in UK Biobank." Current Biology: CB 26 (22): 3083-89. https://www-sciencedirect-com.vunl.idm.oclc.org/science/article/pii/S0960982216311198

Hung, Rayjean J., James D. McKay, Valerie Gaborieau, Paolo Boffetta, Mia Hashibe, David Zaridze, Anush Mukeria, et al. 2008. "A Susceptibility Locus for Lung Cancer Maps to Nicotinic Acetylcholine Receptor Subunit Genes on 15q25." Nature 452 (7187): 633-37. https://pubmed.ncbi.nlm.nih.gov/18385738/

Jansen, Iris E., Jeanne E. Savage, Kyoko Watanabe, Julien Bryois, Dylan M. Williams, Stacy Steinberg, Julia Sealock, et al. 2018. "Genetic Meta-Analysis Identifies 9 Novel Loci and Functional Pathways for Alzheimer's Disease Risk." bioRxiv. https://doi.org/10.1101/258533.

Jencks, Christopher. 1980. "Heredity, Environment, and Public Policy Reconsidered." American Sociological Review 45 (5): 723-36.

Joly, Yann, Gratien Dalpé, Charles Dupras, Bénédicte Bévière-Boyer, Aisling de Paor, Edward S. Dove, Palmira Granados Moreno, et al. 2020. "Establishing the International Genetic Discrimination Observatory." Nature Genetics 52 (5): 466-68.

https://d1wqtxts1xzle7.cloudfront.net/62454266/Joly_et_al_2020_International_Genetic_Discrimina tion_Observatory20200323-30404-rmvmhh.pdf?1584998153=\&response-content-

disposition=inline \%3B+filename\%3DEstablishing the_International_Genetic_D.pdf\&Expires=1594 050598\&Signature $=$ CzdxpvrIKZ-TqZ5C8Ijb4rb-

xapaG4lixOZJe5UcnNcNXohl8ek9bRXS6TKUKGyQCVzcsTw2R3yt8aEgaf1WjQuUmho58kjp OBUxq0Ip6i6smQwQsZxHtaoDqm2MiXZp8Y9io5trSAKqkwlVCgA8 jx $\sim$ fcOJHk7VsvhIzFwa9Jsjh7TYII28jaW5HtqRPOC8mbx OBBHHpKVW5iPDttU1nZOetTtI5d6OyCCJ7nc6I75e9Dq0OAXtoBlycx1KhhA6ZqRbh1Itq5BnuGs QWp8ud6XXwyrWGzD3avSUoScNXC KWqa7tS0SuAqI9dKpQ9s2pmZr7QTCszKJFz0Q \&Key-Pair-Id=APKAJLOHF5GGSLRBV4ZA

Karlsson Linnér, Richard, Pietro Biroli, Edward Kong, S. Fleur W. Meddens, Robbee Wedow, Mark Alan Fontana, Maël Lebreton, et al. 2019. "Genome-Wide Association Analyses of Risk Tolerance and Risky Behaviors in over 1 Million Individuals Identify Hundreds of Loci and Shared Genetic Influences." Nature Genetics 51: 245-57. https://doi.org/10.1038/s41588-018-0309-3

Kevles, Daniel J. 1995. In the Name of Eugenics: Genetics and the Uses of Human Heredity. Harvard University Press.

Kong, Augustine, Gudmar Thorleifsson, Michael L. Frigge, Bjarni J. Vilhjalmsson, Alexander I. Young, Thorgeir E. Thorgeirsson, Stefania Benonisdottir, et al. 2018. "The Nature of Nurture: Effects of Parental Genotypes.” Science 359 (6374): 424-28. https://doi.org/10.1101/219261

Lambert, J. C., C. A. Ibrahim-Verbaas, D. Harold, A. C. Naj, R. Sims, C. Bellenguez, A. L. DeStafano, et al. 2013. "Meta-Analysis of 74,046 Individuals Identifies 11 New Susceptibility Loci for Alzheimer's Disease." Nature Genetics 45 (12): 1452-58. https://ncrad.org/docs/Publications/127_Lambert_2013.pdf

Lander, E. S., and N. J. Schork. 1994. "Genetic Dissection of Complex Traits." Science 265 (5181): 2037-48. http://wwwleland.stanford.edu/ huatang/gene244/readings/Science\%201994\%20Lander.pdf

Lee, James J., Robbee Wedow, Aysu Okbay, Edward Kong, Omeed Maghzian, Meghan Zacher, Tuan Anh Nguyen-Viet, et al. 2018. "Gene Discovery and Polygenic Prediction from a Genome-Wide Association Study of Educational Attainment in 1.1 Million Individuals." Nature Genetics 50 (8): 1112-21. https://doi.org/10.1038/s41588-018-0147-3

Locke, Adam E., Bratati Kahali, Sonja I. Berndt, Anne E. Justice, Tune H. Pers, Felix R. Day, Corey Powell, et al. 2015. "Genetic Studies of Body Mass Index Yield New Insights for Obesity Biology." Nature 518 (7538): 197-206. https://www.ncbi.nlm.nih.gov/pmc/articles/PMC4382211/

Loos, Matthijs J. H. M. van der, Philipp Koellinger, Patrick J. Groenen, Cornelius A. Rietveld, Fernando 
Rivadeneira, Frank van van Rooij, André Uitterlinden, Albert Hofman, and Roy Thurik. 2011. "Candidate Gene Studies and the Quest for the Entrepreneurial Gene." Small Business Economics 37 (3): 269-75. https://core.ac.uk/reader/81883844

Martin, Alicia R., Masahiro Kanai, Yoichiro Kamatani, Yukinori Okada, Benjamin M. Neale, and Mark J. Daly. 2019. "Clinical Use of Current Polygenic Risk Scores May Exacerbate Health Disparities."

Nature Genetics 51 (4): 584-91. https://www.ncbi.nlm.nih.gov/pmc/articles/PMC6563838/

Mills, Melinda C., and Charles Rahal. 2019. "A Scientometric Review of Genome-Wide Association Studies.” Communications Biology 2 (January): 9. https://doi.org/10.1038/s42003-018-0261-x

Moore, T. Owens. 2018. "Stamped from the Beginning: The Definitive History of Racist Ideas in America, by Ibram X. Kendi." The Black Scholar 48 (3): 71-74.

Mostafavi, Hakhamanesh, Arbel Harpak, Ipsita Agarwal, Dalton Conley, Jonathan K. Pritchard, and Molly Przeworski. 2020. "Variable Prediction Accuracy of Polygenic Scores within an Ancestry Group." eLife 9 (January). https://doi.org/10.7554/eLife.48376

Nozick, Robert. 1974. Anarchy, State, and Utopia. New York: Basic Books.

Okbay, Aysu, Bart M. L. Baselmans, Jan-Emmanuel De Neve, Patrick Turley, Michel G. Nivard, Mark Alan Fontana, S. Fleur W. Meddens, et al. 2016. "Genetic Variants Associated with Subjective WellBeing, Depressive Symptoms, and Neuroticism Identified through Genome-Wide Analyses." Nature Genetics 48 (6): 624-33. http://dx.doi.org/10.1038/ng.3552

Pardiñas, Antonio F., Peter Holmans, Andrew J. Pocklington, Valentina Escott-Price, Stephan Ripke, Noa Carrera, Sophie E. Legge, et al. 2018. "Common Schizophrenia Alleles Are Enriched in MutationIntolerant Genes and in Regions under Strong Background Selection." Nature Genetics 50 (3): 38189. http://orca.cf.ac.uk/96134/1/068593.full.pdf

Piketty, Thomas. 1995. "Social Mobility and Redistributive Politics." The Quarterly Journal of Economics 110 (3): 551-84. https://www.haverford.edu/sites/default/files/Piketty1995.pdf

Piotrowska, Patrycja J., Christopher B. Stride, Simone E. Croft, and Richard Rowe. 2015. "Socioeconomic Status and Antisocial Behaviour among Children and Adolescents: A Systematic Review and Meta-Analysis." Clinical Psychology Review 35 (February): 47-55. https://www.sciencedirect.com/science/article/pii/S0272735814001652?via\%3Dihub

Polderman, Tinca J. C., Beben Benyamin, Christiaan A. de Leeuw, Patrick F. Sullivan, Arjen van Bochoven, Peter M. Visscher, and Danielle Posthuma. 2015. "Meta-Analysis of the Heritability of Human Traits Based on Fifty Years of Twin Studies." Nature Genetics 47: 702-9. https://www.researchgate.net/profile/Beben_Benyamin/publication/276922271_Metaanalysis_of_the_heritability_of_human_traits_based_on_fifty_years_of_twin_studies/links/555dd99 b08ae8c0cab2b9b9a.pdf

Rawls, John. 1999. A Theory of Justice. Belknap Press of Harvard University Press.

Rietveld, Cornelius A., Sarah E. Medland, Jaime Derringer, Jian Yang, Tõnu Esko, Nicolas W. Martin, Harm-Jan Westra, et al. 2013. "GWAS of 126,559 Individuals Identifies Genetic Variants Associated with Educational Attainment." Science 340 (6139): 1467-71. https://www.ncbi.nlm.nih.gov/pmc/articles/PMC3751588/

Ripke, Stephan, Benjamin M. Neale, Aiden Corvin, James T. R. Walters, Kai-How Farh, Peter A. Holmans, Phil Lee, et al. 2014. "Biological Insights from 108 Schizophrenia-Associated Genetic Loci." Nature 511 (7510): 421-27. https://www.nature.com/articles/nature13595

Roemer, John E. 1998. Equality of Opportunity. Harvard University Press.

Stringhini, Silvia, Cristian Carmeli, Markus Jokela, Mauricio Avendaño, Peter Muennig, Florence Guida, Fulvio Ricceri, et al. 2017. "Socioeconomic Status and the 25× 25 Risk Factors as Determinants of Premature Mortality: A Multicohort Study and Meta-Analysis of 1.7 Million Men and Women." The Lancet 389 (10075): 1229-37. https://www.cop-cv.org/db/docu/170214092306hIK9sRNEQNbA.pdf

Taubman, Paul. 1976. "The Determinants of Earnings: Genetics, Family, and Other Environments: A Study of White Male Twins." The American Economic Review 66 (5): 858-70.

https://www.gwern.net/docs/genetics/heritable/1976-taubman.pdf

Thorgeirsson, Thorgeir E., Frank Geller, Patrick Sulem, Thorunn Rafnar, Anna Wiste, Kristinn P. 
Magnusson, Andrei Manolescu, et al. 2008. "A Variant Associated with Nicotine Dependence, Lung Cancer and Peripheral Arterial Disease.” Nature 452 (7187): 638-42.

https://www.ncbi.nlm.nih.gov/pmc/articles/PMC4539558/

Turkheimer, Eric. 2000. "Three Laws of Behavior Genetics and What They Mean." Current Directions in Psychological Science 9 (5): 160-64. https://www.academia.edu/download/5030966/cd9_5_5.pdf

Turley, Patrick, Raymond K. Walters, Omeed Maghzian, Aysu Okbay, James J. Lee, Mark Alan Fontana, Tuan Anh Nguyen-Viet, et al. 2018. "Multi-Trait Analysis of Genome-Wide Association Summary Statistics Using MTAG." Nature Genetics 50 (2): 229-37. https://europepmc.org/articles/pmc5805593/bin/nihms918509-supplement-3.pdf

Vassos, Evangelos, Marta Di Forti, Jonathan Coleman, Conrad Iyegbe, Diana Prata, Jack Euesden, Paul O'Reilly, et al. 2017. "An Examination of Polygenic Score Risk Prediction in Individuals With FirstEpisode Psychosis.” Biological Psychiatry 81 (6): 470-77.

http:/www.biologicalpsychiatryjournal.com/article/S0006322316326646/pdf

Visscher, Peter M., William G. Hill, and Naomi R. Wray. 2008. "Heritability in the Genomics Era-Concepts and Misconceptions." Nature Reviews. Genetics 9 (4): 255-66. https://www.sdu.dk//media/files/om_sdu/institutter/ist/epid/courses/twins/papers/visscher.pdf

Visscher, Peter M., Naomi R. Wray, Qian Zhang, Pamela Sklar, Mark I. McCarthy, Matthew A. Brown, and Jian Yang. 2017. "10 Years of GWAS Discovery: Biology, Function, and Translation." American Journal of Human Genetics 101 (1): 5-22. https://renovatiobiomedica.com/wpcontent/uploads/2017/07/10-a\%C3\%B1os-GWAS.pdf

Vlaming, Ronald de, Aysu Okbay, Cornelius A. Rietveld, Magnus Johannesson, Patrik K. E. Magnusson, André G. Uitterlinden, Frank J. A. van Rooij, et al. 2017. "Meta-GWAS Accuracy and Power (MetaGAP) Calculator Shows That Hiding Heritability Is Partially due to Imperfect Genetic Correlations across Studies." PLoS Genetics 13 (1). https://doi.org/10.1371/journal.pgen.1006495.

Wilkinson, Richard G., and Michael Marmot. 2003. Social Determinants of Health: The Solid Facts. World Health Organization.

Winkler, Thomas W., Felix R. Day, Damien C. Croteau-Chonka, Andrew R. Wood, Adam E. Locke, Reedik Mägi, Teresa Ferreira, et al. 2014. "Quality Control and Conduct of Genome-Wide Association Meta-Analyses.” Nature Protocols 9 (5): 1192-1212. http://www.academia.edu/download/35918804/nprot14_Winkler_et_al_Giant-QC.pdf

Wood, Andrew R., Tonu Esko, Jian Yang, Sailaja Vedantam, Tune H. Pers, ..., Timothy M. Frayling, et al. 2014. "Defining the Role of Common Variation in the Genomic and Biological Architecture of Adult Human Height." Nature Genetics 46 (11): 1173-86. http://citeseerx.ist.psu.edu/viewdoc/download?doi=10.1.1.792.7246\&rep=rep1\&type=pdf

Yengo, Loic, Julia Sidorenko, Kathryn E. Kemper, Zhili Zheng, Andrew R. Wood, Michael N. Weedon, Timothy M. Frayling, et al. 2018. "Meta-Analysis of Genome-Wide Association Studies for Height and Body Mass Index in 700,000 Individuals of European Ancestry." Human Molecular Genetics 27: 3641-49. https://europepmc.org/article/med/30124842

Young, Alexander I., Stefania Benonisdottir, Molly Przeworski, and Augustine Kong. 2019.

"Deconstructing the Sources of Genotype-Phenotype Associations in Humans." Science 365 (6460): 1396-1400. https://europepmc.org/article/pmc/pmc6894903 